\title{
Catalyst-Controlled Regioselective Synthesis of \\ Benzotriazlolodiazepin-7-ones \\ and
}

\section{Benzotriazolodiazocin-8-ones}

Kai-Chi Chen, ${ }^{\dagger}$ Indrajeet J. Barve ${ }^{\dagger \dagger}$ and Chung-Ming Sun ${ }^{\dagger \S^{*}}$

'Department of Applied Chemistry, 1001 Ta-Hsueh Road, National Chiao-Tung University, Hsinchu 300-10, Taiwan, ROC

${ }^{\S}$ Department of Medicinal and Applied Chemistry, Kaohsiung Medical University, 100, Shih-Chuan $1^{\text {st }}$ Road, Kaohsiung 807-08, Taiwan, ROC. ${ }^{t}$ Department of Chemistry, MES Abasaheb Garware College, Pune, India

Table of Contents

\begin{tabular}{|l|l|}
\hline General Methods & S2 \\
\hline A representative procedure for the synthesis of amide 1a & S3-S5 \\
\hline Characterization data of compounds S2-S7 and amide 1a & S6-S7 \\
\hline Optimization Table S1 & S8 \\
\hline A representative procedure for the synthesis of 2a and 3a & S9-S11 \\
\hline Characterization data of compounds 2b-2n and 3b-3k & S12-S23 \\
\hline Spectral data of compounds S2-S7 and amide 1a & S24-S38 \\
\hline Spectral data of compounds 2a-2n & S39-S122 \\
\hline Spectral data of compounds 3a-3k & S123-S188 \\
\hline X-ray crystallographic data of compound 2a & S189-S197 \\
\hline X-ray crystallographic data of compound 3a & S198-S206 \\
\hline
\end{tabular}


${ }^{1} \mathrm{H}$ NMR (400 MHz) and ${ }^{13} \mathrm{C}$ NMR (101 MHz) spectra were recorded on 400-MR automated spectrometer. Chemical shifts are reported in parts per million (ppm) on the $\delta$ scale from an internal standard (TMS). Analytical thin-layer chromatography (TLC) was performed using $0.25 \mathrm{~mm}$ silica gel-coated Kiselgel $60 \mathrm{~F}_{254}$ plates. Flash chromatography was performed using the indicated solvent and silica gel 60 (Merck, 230-400 mesh). High-resolution mass spectra (HRMS) were recorded in ESI mode using TOF mass spectrometer. Unless otherwise stated, oil bath was used as a heat source for all reactions. All materials were purchased from commercial sources and used without further purification.

A representative procedure for the synthesis of



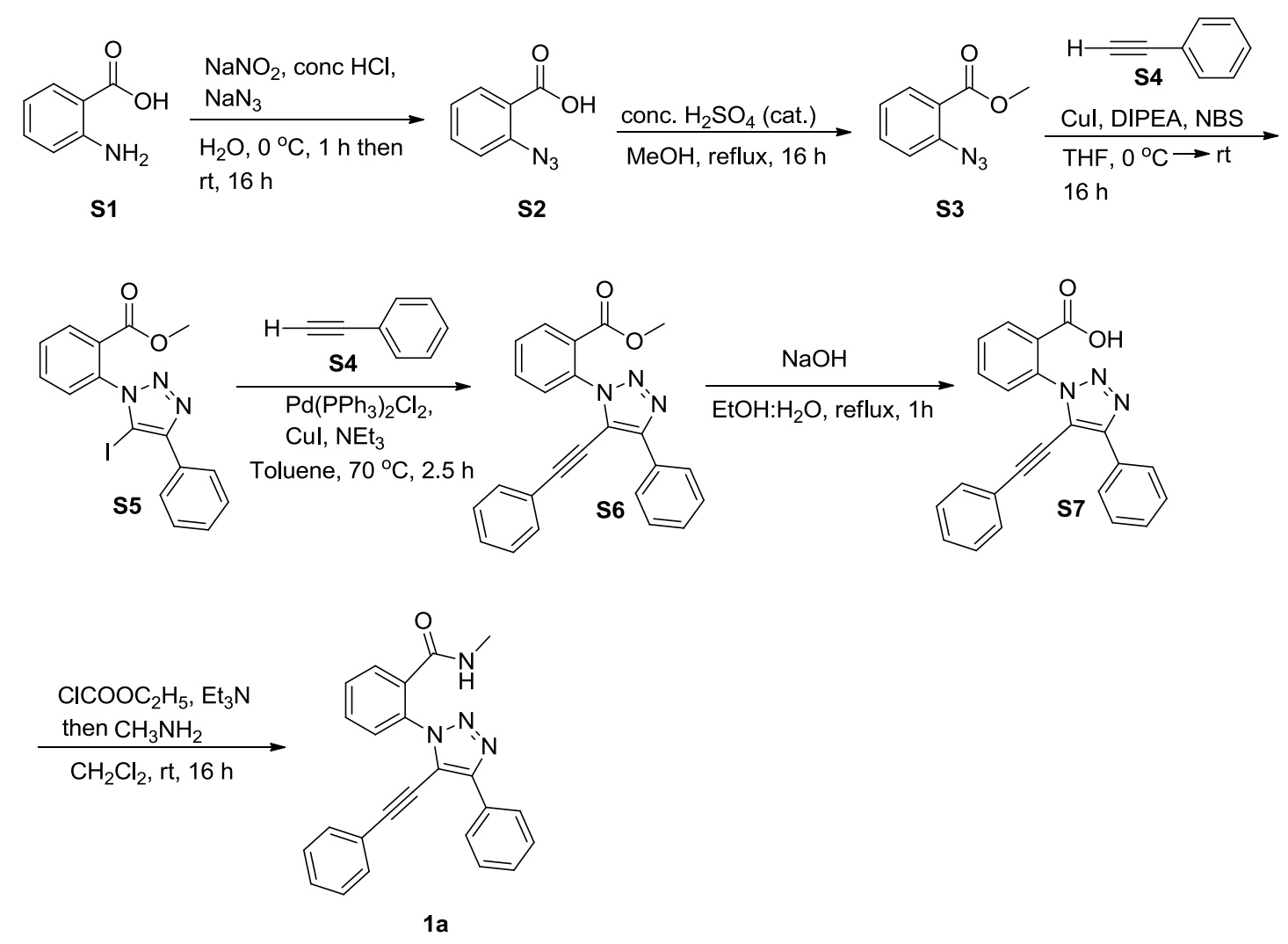

Scheme S1. Synthesis of amide 1a (compounds $\mathbf{S 2}$ and $\mathbf{S 3}$ were prepared according to literature method. ${ }^{1,2}$ )

A suspension of anthranilic acid $\mathbf{S 1}(3 \mathrm{~g}, 21.8 \mathrm{mmol})$ in water $(15 \mathrm{~mL})$ was cooled to $-5{ }^{\circ} \mathrm{C}$, then conc. $\mathrm{HCl}(7.5 \mathrm{~mL})$ and a solution of sodium nitrite $(1.81 \mathrm{~g}, 26.2 \mathrm{mmol})$ in water $(15 \mathrm{~mL})$ was added dropwise. The above solution was stirred at $-5{ }^{\circ} \mathrm{C}$ for 1 $\mathrm{h}$ and poured into a solution of sodium azide $(1.7 \mathrm{~g}, 26.2 \mathrm{mmol})$ in water $(25 \mathrm{~mL})$ at $-5{ }^{\circ} \mathrm{C}$. The resulting pale yellow solid was stirred for $16 \mathrm{~h}$. The solid was filtered through Buchner funnel, washed with water $(200 \mathrm{~mL})$ and dried to afford 2-azido benzoic acid S2 as an off-white solid (3.4 g, $95 \%$ ). To a solution of 2-azido benzoic acid S2 (3 g, $18.3 \mathrm{mmol})$ in methanol was added conc. $\mathrm{H}_{2} \mathrm{SO}_{4}(1.3 \mathrm{~mL})$ and the reaction mixture was refluxed for $16 \mathrm{~h}$. The solvent was evaporated; the crude 
reaction mixture was neutralized with sat. $\mathrm{NaHCO}_{3}$ and extracted with ethyl acetate (3 x $20 \mathrm{~mL}$ ). The combined organic layers were washed with brine solution, dried over $\mathrm{MgSO}_{4}$ and concentrated in vacuo to afford methyl 2-azidobenzoate $\mathbf{S 3}$ as brown oil (3.2 g, 98\%). A solution of NBS (1.09 g, $6.15 \mathrm{mmol})$ in THF (40 mL) was cooled to $0{ }^{\circ} \mathrm{C}$ and $\mathrm{CuI}(1.07 \mathrm{~g}, 5.64 \mathrm{mmol})$, methyl 2-azidobenzoate $\mathbf{S 3}$ (1 g, 5.64 mmol), phenyl acetylene $(0.57 \mathrm{~g}, 5.64 \mathrm{mmol})$ and DIPEA $(0.98 \mathrm{~mL}, 5.64 \mathrm{mmol})$ were added. The resulting reaction mixture was stirred at room temperature for $16 \mathrm{~h}$. The solvent was evaporated; the reaction mixture was diluted with water $(40 \mathrm{~mL})$ and extracted with ethyl acetate $(3 \times 25 \mathrm{~mL})$. The combined organic layers were washed with brine solution, dried over $\mathrm{MgSO}_{4}$ and concentrated under reduced pressure. The crude was purified by flash column chromatography (50-70\% ethyl $\begin{array}{llll}\text { acetate } & \text { h } & \text { hexanes) }\end{array}$ 2-(5-iodo-4-phenyl-1H-1,2,3-triazol-1-yl)benzoate $\mathbf{S 5}$ as a white solid (1.82 g, 80\%). To a mixture of methyl 2-(5-iodo-4-phenyl-1H-1,2,3-triazol-1-yl)benzoate S5 (1 g, $2.46 \mathrm{mmol})$ in toluene was added phenyl acetylene (0.37 g, $3.7 \mathrm{mmol}), \mathrm{Pd}\left(\mathrm{PPh}_{3}\right)_{2} \mathrm{Cl}_{2}$ (0.17 g, $0.24 \mathrm{mmol}), \mathrm{CuI}(0.093 \mathrm{~g}, 0.49 \mathrm{mmol})$ and triethyl amine $(2 \mathrm{~mL}, 14.8 \mathrm{mmol})$ under nitrogen atmosphere and the reaction mixture was heated at $70{ }^{\circ} \mathrm{C}$ for $2.5 \mathrm{~h}$. The solvent was evaporated, the crude reaction mixture was diluted with water (30 $\mathrm{mL})$ and extracted with ethyl acetate $(3 \times 25 \mathrm{~mL})$. The combined organic layers were washed with brine solution, dried over $\mathrm{MgSO}_{4}$ and concentrated. The crude product was purified by flash column chromatography (5-7\% ethyl acetate in hexanes) to obtain methyl 2-(4-phenyl-5-(phenylethynyl)-1H-1,2,3-triazol-1-yl)benzoate S6 as a pale yellow solid $(0.8 \mathrm{~g}, \quad 90 \%)$. To a mixture of methyl 2-(4-phenyl-5-(phenylethynyl)-1H-1,2,3-triazol-1-yl)benzoate S6 (0.4 g, 1.05 mmol) in ethanol : $\mathrm{H}_{2} \mathrm{O}(1: 1 ; 10 \mathrm{~mL})$ was added $\mathrm{NaOH}(0.4 \mathrm{~g})$ and the reaction was refluxed for $1 \mathrm{~h}$. The solvent was evaporated. The crude reaction mixture was 
neutralized with $1 \mathrm{~N} \mathrm{HCl}$ and extracted with ethyl acetate $(3 \times 15 \mathrm{~mL})$. The combined organic layers were washed with brine solution, dried over $\mathrm{MgSO}_{4}$ and $\begin{array}{llll}\text { concentrated } & \text { in } & \text { vacuo } & \text { to }\end{array}$ 2-(4-phenyl-5-(phenylethynyl)-1H-1,2,3-triazol-1-yl)benzoic acid S8 as an off-white

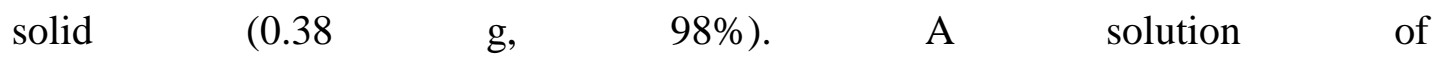
2-(4-phenyl-5-(phenylethynyl)-1H-1,2,3-triazol-1-yl)benzoic acid S8 (0.35 g, 0.96 mmol $)$ in dichloromethane was cooled at $-5^{\circ} \mathrm{C}$, triethylamine $(0.15 \mathrm{~g}, 1.44 \mathrm{mmol})$ was added under nitrogen atmosphere and the reaction mixture was stirred for $15 \mathrm{~min}$. To the above reaction mixture ethyl chloroformate $(0.13 \mathrm{~g}, 1.15 \mathrm{mmol})$ was added slowly in a dropwise manner and the reaction mixture was stirred for $1 \mathrm{~h}$. To this reaction mixture methylamine $(0.44 \mathrm{~g}, 1.42 \mathrm{mmol})$ was added and the reaction was stirred at room temperature for $16 \mathrm{~h}$. After completion, the reaction mixture was diluted with dichloromethane and washed with water $(2 \times 25 \mathrm{~mL})$. The organic layer was washed with brine solution, dried over $\mathrm{MgSO}_{4}$ and concentrated. The crude product was purified by flash column chromatography (12-15\% ethyl acetate in hexanes) to obtain $N$-methyl-2-(4-phenyl-5-(phenylethynyl)-1H-1,2,3-triazol-1-yl)benzamide $\mathbf{1 a}$ as a white solid $(0.33 \mathrm{~g}, 91 \%)$.

\section{References}

1. Hahn, F. E.; Langenhahn, V.; Meier, N.; Lügger, T.; Fehlhammer, W. P. Chem. Eur. J. 2003, 9, 704-712.

2. Alcaide, B.; Almendros, P.; Lázaro-Milla, C. Chem. Commun. 2015, 51, 69926995. 
${ }^{1} \mathrm{H}$ NMR (400 MHz, Acetone- $\left.d_{6}\right) \delta 11.35(\mathrm{bs}, 1 \mathrm{H}), 7.90(\mathrm{~d}, J=9.3 \mathrm{~Hz}, 1 \mathrm{H}), 7.63(\mathrm{t}$,

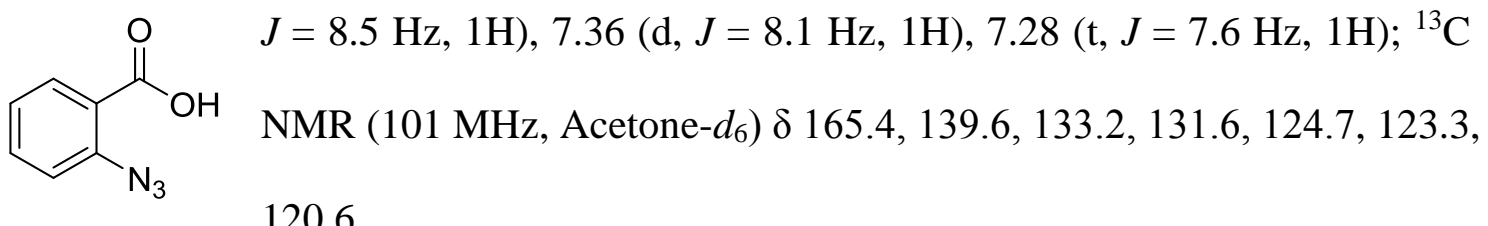
120.6 .

\section{Methyl 2-azidobenzoate (S3)}

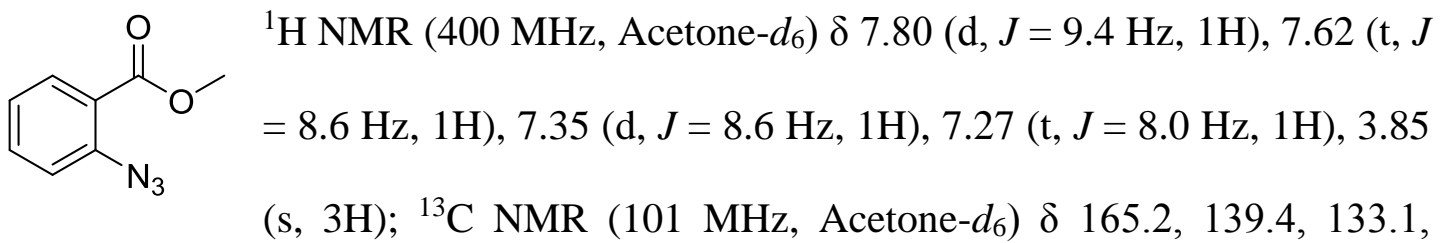

131.2, 124.7, 123.2, 120.4, 51.5; LRMS (ESI) m/z: $200.2(\mathrm{M}+\mathrm{Na})^{+}$

\section{Methyl 2-(5-iodo-4-phenyl-1H-1,2,3-triazol-1-yl)benzoate (S5)}

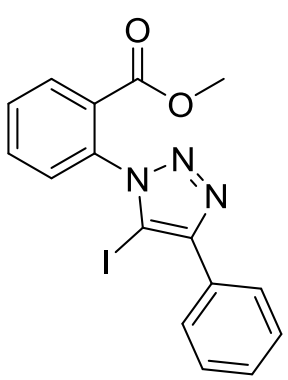

${ }^{1} \mathrm{H}$ NMR $\left(400 \mathrm{MHz}, \mathrm{CDCl}_{3}\right) \delta 8.18(\mathrm{~d}, J=7.7 \mathrm{~Hz}, 1 \mathrm{H}), 8.04(\mathrm{~d}$, $J=8.1 \mathrm{~Hz}, 2 \mathrm{H}), 7.75(\mathrm{t}, J=7.6 \mathrm{~Hz}, 1 \mathrm{H}), 7.68(\mathrm{t}, J=7.6 \mathrm{~Hz}, 1 \mathrm{H})$, $7.52-7.38(\mathrm{~m}, 4 \mathrm{H}), 3.66(\mathrm{~s}, 3 \mathrm{H}) ;{ }^{13} \mathrm{C} \mathrm{NMR}\left(101 \mathrm{MHz}, \mathrm{CDCl}_{3}\right)$ $\delta 164.4,149.4,136.3,133.1,131.7,130.8,130.0,129.3,128.6$, 128.5, 128.5, 127.4, 79.9, 52.6; LRMS (ESI, $m / z): 406.1$ $(\mathrm{M}+\mathrm{H})^{+}$; HRMS (ESI, m/z) calculated for $\mathrm{C}_{16} \mathrm{H}_{13} \mathrm{IN}_{3} \mathrm{O}_{2}[\mathrm{M}+\mathrm{H}]^{+} 406.0052$, found 406.0047.

Methyl 2-(4-phenyl-5-(phenylethynyl)-1H-1,2,3-triazol-1-yl)benzoate (S6)

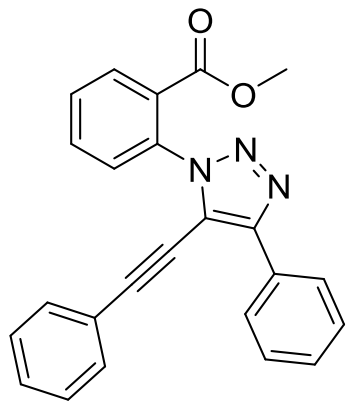
${ }^{1} \mathrm{H}$ NMR $\left(400 \mathrm{MHz}, \mathrm{CDCl}_{3}\right) \delta 8.27(\mathrm{~d}, J=7.5 \mathrm{~Hz}, 2 \mathrm{H}), 8.13$ $(\mathrm{d}, J=7.5 \mathrm{~Hz}, 1 \mathrm{H}), 7.74(\mathrm{t}, J=7.5 \mathrm{~Hz}, 1 \mathrm{H}), 7.65(\mathrm{t}, J=6.3$ $\mathrm{Hz}, 3 \mathrm{H}), 7.49$ (t, $J=7.6 \mathrm{~Hz}, 2 \mathrm{H}), 7.36(\mathrm{~m}, 5 \mathrm{H}), 3.68(\mathrm{~s}, 3 \mathrm{H})$; ${ }^{13} \mathrm{C}$ NMR $\left(101 \mathrm{MHz}, \mathrm{CDCl}_{3}\right) \delta 165.4,147.4,135.1,132.8$, $131.4,130.2,130.1,129.6,128.7,128.5,128.04,127.9,126.3$, 121.2, 118.9, 101.9, 75.4, 52.6; LRMS (ESI, $m / z): 380.3(\mathrm{M}+\mathrm{H})^{+}$; HRMS (ESI, $\left.m / z\right)$ calculated for $\mathrm{C}_{24} \mathrm{H}_{18} \mathrm{~N}_{3} \mathrm{O}_{2}[\mathrm{M}+\mathrm{H}]^{+} 380.1399$, found 380.1394 . 


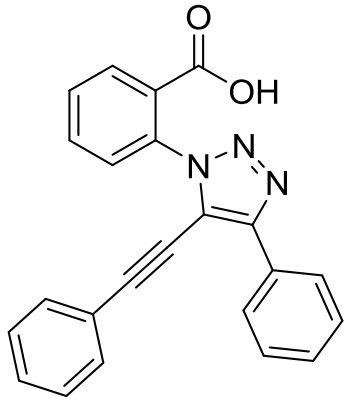

${ }^{1} \mathrm{H}$ NMR $\left(400 \mathrm{MHz}, \mathrm{CDCl}_{3}\right) \delta 8.22(\mathrm{~d}, J=7.8 \mathrm{~Hz}, 2 \mathrm{H}), 8.16$ (d, $J=7.7 \mathrm{~Hz}, 1 \mathrm{H}), 7.74(\mathrm{t}, J=7.7 \mathrm{~Hz}, 1 \mathrm{H}), 7.60(\mathrm{~m}, 3 \mathrm{H})$, $7.46(\mathrm{t}, J=7.4 \mathrm{~Hz}, 2 \mathrm{H}), 7.33(\mathrm{~m}, 5 \mathrm{H}), 4.12(\mathrm{bs}, 1 \mathrm{H}) ;{ }^{13} \mathrm{C}$ NMR (101 MHz, $\left.\mathrm{CDCl}_{3}\right) \delta 168.1,147.3,135.4,133.2,132.1$, $132.1,132.0,131.9,131.5,130.3,130.0,129.5,128.6,128.5$, 128.4, 128.2, 126.4, 120.2, 102.0, 75.4; LRMS (ESI, $\mathrm{m} / \mathrm{z}$ ): 366.3; HRMS (ESI, $\mathrm{m} / \mathrm{z}$ ) calculated for $\mathrm{C}_{23} \mathrm{H}_{16} \mathrm{~N}_{3} \mathrm{O}_{2}[\mathrm{M}+\mathrm{H}]^{+}$366.1243, found 366.1237.

$N$-methyl-2-(4-phenyl-5-(phenylethynyl)-1H-1,2,3-triazol-1-yl)benzamide (1a)<smiles>CNC(=O)c1ccccc1-n1nnc(-c2ccccc2)c1C#Cc1ccccc1</smiles>
${ }^{1} \mathrm{H}$ NMR (400 MHz, $\left.\mathrm{CDCl}_{3}\right) \delta 8.25(\mathrm{~d}, J=6.6 \mathrm{~Hz}, 1 \mathrm{H}), 7.86$ (s, 1H), 7.61-7.47 (m, 5H), $7.40-7.33(\mathrm{~m}, 5 \mathrm{H}), 6.10(\mathrm{~s}, 1 \mathrm{H})$, $2.78(\mathrm{~s}, 3 \mathrm{H}) ;{ }^{13} \mathrm{C} \mathrm{NMR}\left(101 \mathrm{MHz}, \mathrm{CDCl}_{3}\right) \delta$ 166.2, 147.6, $133.8,132.9,131.6,130.9,130.5,129.9,129.8,129.6,128.8$, $128.7,128.5,127.5,126.3,121.1,119.3,102.6,75.0,26.9$; HRMS (ESI, $m / z$ ) calculated for $\mathrm{C}_{24} \mathrm{H}_{19} \mathrm{~N}_{4} \mathrm{O}[\mathrm{M}+\mathrm{H}]^{+} 379.1559$, found 379.1561 .

Table S1. Optimization of the reaction conditions ${ }^{a}$ 


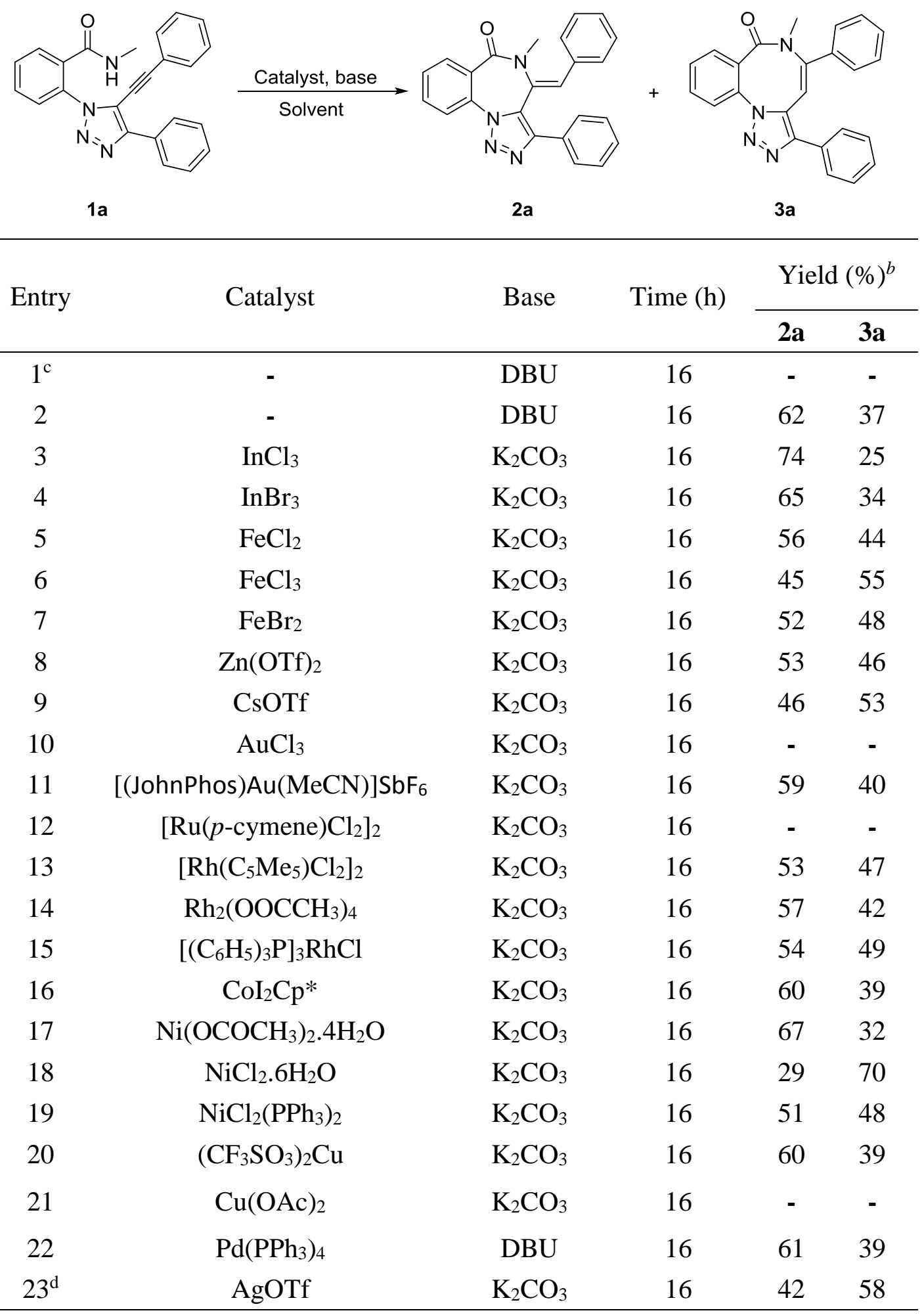

${ }^{a}$ Reaction conditions: 1a (1 equiv), catalyst (10 mol\%), base ( 3 equiv), $\mathrm{CH}_{3} \mathrm{CN}$ (5 $\mathrm{mL}), 110{ }^{\circ} \mathrm{C}$, sealed tube, $16 \mathrm{~h},{ }^{b}$ Isolated yield, ${ }^{c}$ refluxed at $85{ }^{\circ} \mathrm{C},{ }^{d}$ reaction was carried out in toluene at $130{ }^{\circ} \mathrm{C}$.

A representative procedure for the synthesis of 
(Z)-4-benzylidene-5-methyl-3-phenyl-4,5-dihydro-6H-benzo[f][1,2,3]triazolo[1,5

$-a][1,4]$ diazepin-6-one (2a)

A sealed tube was charged with $N$-methyl-2-(4-phenyl-5-(phenylethynyl)-1H-1,2,3-triazol-1-yl)benzamide $\mathbf{1 a} \quad(0.1$ $\mathrm{g}, \quad 0.264 \mathrm{mmol})$, tetras(triphenylphosphine)palladium $(0.046 \mathrm{~g}, 0.04 \mathrm{mmol})$, potassium carbonate $(0.16 \mathrm{~g}, 1.16 \mathrm{mmol})$ and acetonitrile $(4 \mathrm{~mL})$. The reaction mixture was heated in an oil bath at $110{ }^{\circ} \mathrm{C}$ for $16 \mathrm{~h}$. After completion of the reaction, the reaction mixture was filtered through a thin layer of celite bed, and the filtrate was concentrated under reduced pressure. The crude product was purified by flash column chromatography (8-10\% ethyl acetate in hexanes) to afford (Z)-4-benzylidene-5-methyl-3-phenyl-4,5-dihydro-6H-benzo[f][1,2,3]triazolo[1,5- $a]$ [1,4]diazepin-6-one 2a.

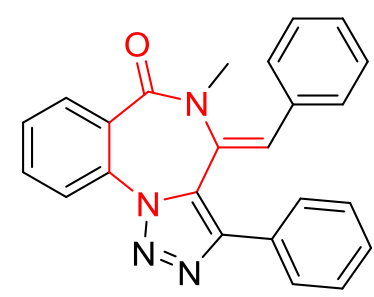

White solid (76 mg, $76 \%$ ); mp 90-92 ${ }^{\circ} \mathrm{C} ;{ }^{1} \mathrm{H} \mathrm{NMR}(400 \mathrm{MHz}$, $\left.\mathrm{CDCl}_{3}\right) \delta 8.16(\mathrm{~d}, J=7.6 \mathrm{~Hz}, 1 \mathrm{H}), 8.08(\mathrm{~d}, J=8.1 \mathrm{~Hz}, 1 \mathrm{H})$, $7.97(\mathrm{~d}, J=7.4 \mathrm{~Hz}, 2 \mathrm{H}), 7.69(\mathrm{t}, J=7.6 \mathrm{~Hz}, 1 \mathrm{H}), 7.56(\mathrm{t}, J=$ $7.6 \mathrm{~Hz}, 1 \mathrm{H}), 7.48(\mathrm{t}, J=7.4 \mathrm{~Hz}, 2 \mathrm{H}), 7.43-7.36(\mathrm{~m}, 4 \mathrm{H})$, $7.31(\mathrm{~d}, J=7.7 \mathrm{~Hz}, 2 \mathrm{H}), 6.58(\mathrm{~s}, 1 \mathrm{H}), 3.31(\mathrm{~s}, 3 \mathrm{H}) ;{ }^{13} \mathrm{C} \mathrm{NMR}\left(101 \mathrm{MHz}, \mathrm{CDCl}_{3}\right) \delta$ $165.9,142.6,133.4,133.3,132.8,132.4,132.0,129.8,129.7,129.1,128.9,128.9$, 128.7, 126.7, 126.5, 124.7, 122.4, 35.51; LRMS (ESI) m/z: $379.1(\mathrm{M}+\mathrm{H})^{+}$; HRMS (ESI) calcd for $\mathrm{C}_{24} \mathrm{H}_{18} \mathrm{~N}_{4} \mathrm{O}$ m/z: 379.1553; Found $379.1551(\mathrm{M}+\mathrm{H})^{+}$.

A representative procedure for the synthesis of (Z)-6-methyl-3,5-diphenylbenzo $[g][1,2,3]$ triazolo[1,5-a $][1,5]$ diazocin-7(6H)-one (3a)

A sealed tube was charged with $N$-methyl-2-(4-phenyl-5-(phenylethynyl)-1H-1,2,3-triazol-1-yl)benzamide $\mathbf{1 a} \quad(0.1$ 
$\mathrm{g}, \quad 0.264 \mathrm{mmol}), \quad$ silver trifluoromethanesulfonate $(0.007 \mathrm{~g}, 0.027 \mathrm{mmol})$, potassium carbonate $(0.16 \mathrm{~g}, 1.16 \mathrm{mmol})$ and acetonitrile $(4 \mathrm{~mL})$. The reaction mixture was heated in an oil bath at $110{ }^{\circ} \mathrm{C}$ for $16 \mathrm{~h}$. After completion of the reaction, the reaction mixture was filtered through a thin layer of celite bed, and the filtrate was concentrated under reduced pressure. The crude product was purified by flash column chromatography (8-10\% ethyl acetate in hexanes) to afford (Z)-6-methyl-3,5-diphenylbenzo[ $g][1,2,3]$ triazolo[1,5-a][1,5]diazocin-7(6H)-one 3a.<smiles>CN1C(=O)c2ccccc2-n2nnc(-c3ccccc3)c2/C=C\1c1ccccc1</smiles>

Yellow solid (81 mg, $81 \%$ ); mp 99-101 ${ }^{\circ} \mathrm{C} ;{ }^{1} \mathrm{H}$ NMR (400 $\left.\mathrm{MHz}, \mathrm{CDCl}_{3}\right) \delta 7.97(\mathrm{~d}, J=7.3 \mathrm{~Hz}, 2 \mathrm{H}), 7.77-7.72(\mathrm{~m}, 1 \mathrm{H})$, $7.58-7.56(\mathrm{~m}, 2 \mathrm{H}), 7.54-7.50(\mathrm{~m}, 5 \mathrm{H}), 7.49-7.39(\mathrm{~m}, 4 \mathrm{H})$,

$6.68(\mathrm{~s}, 1 \mathrm{H}), 3.06(\mathrm{~s}, 3 \mathrm{H}) ;{ }^{13} \mathrm{C} \mathrm{NMR}\left(101 \mathrm{MHz}, \mathrm{CDCl}_{3}\right) \delta$ $167.2,148.9,143.6,134.1,132.5,132.2,131.1,130.6,130.3,130.2,129.4,129.0$ 128.9, 128.6, 128.4, 128.3, 126.5, 126.3, 125.9, 122.3, 34.9; LRMS (ESI) $m / z: 379.1$ $(\mathrm{M}+\mathrm{H})^{+}$; HRMS (ESI) calcd for $\mathrm{C}_{24} \mathrm{H}_{18} \mathrm{~N}_{4} \mathrm{O} \mathrm{m} / \mathrm{z}$ : 379.1553 ; found 379.1551 $(\mathrm{M}+\mathrm{H})^{+}$; IR $\left(\mathrm{cm}^{-1}\right.$, neat) 3056, 2915, 1647, 1381, 774, 595.

Representative procedure at $1 \mathrm{mmol}$ scale for the synthesis of 
(Z)-4-benzylidene-5-methyl-3-phenyl-4,5-dihydro-6H-benzo[f][1,2,3]triazolo[1,5

$-a][1,4]$ diazepin-6-one (2a)

A sealed tube was charged with $N$-methyl-2-(4-phenyl-5-(phenylethynyl)-1H-1,2,3-triazol-1-yl)benzamide $1 \mathbf{1 a}(0.5$ $\mathrm{g}, 1.321 \mathrm{mmol})$, tetras(triphenylphosphine)palladium (0.152 $\mathrm{g}, 0.132 \mathrm{mmol})$, potassium carbonate $(0.547 \mathrm{~g}, 3.963 \mathrm{mmol})$ and acetonitrile $(8 \mathrm{~mL})$. The reaction mixture was heated in an oil bath at $110{ }^{\circ} \mathrm{C}$ for $16 \mathrm{~h}$. After completion of the reaction, the reaction mixture was filtered through a thin layer of celite bed, and the filtrate was concentrated under reduced pressure. The crude product was purified by flash column chromatography $(8-10 \%$ ethyl acetate in hexanes) to afford (Z)-4-benzylidene-5-methyl-3-phenyl-4,5-dihydro-6H-benzo[f][1,2,3]triazolo[1,5- $a]$ [1,4]diazepin-6-one 2a as a white solid (375 mg, $75 \%$ ).

Representative procedure at $1 \mathrm{mmol}$ scale for the synthesis of (Z)-6-methyl-3,5-diphenylbenzo[g][1,2,3]triazolo[1,5-a][1,5]diazocin-7(6H)-one (3a)

A sealed tube was charged with $N$-methyl-2-(4-phenyl-5-(phenylethynyl)-1H-1,2,3-triazol-1-yl)benzamide $\mathbf{1 a} \quad(0.5$ $\mathrm{g}, \quad 1.321 \mathrm{mmol})$, silver trifluoromethanesulfonate $(0.152 \mathrm{~g}, 0.132 \mathrm{mmol})$, potassium carbonate $(0.547 \mathrm{~g}, 3.963 \mathrm{mmol})$ and acetonitrile $(8 \mathrm{~mL})$. The reaction mixture was heated in an oil bath at $110{ }^{\circ} \mathrm{C}$ for $16 \mathrm{~h}$. After completion of the reaction, the reaction mixture was filtered through a thin layer of celite bed, and the filtrate was concentrated under reduced pressure. The crude product was purified by flash column chromatography (8-10\% ethyl acetate in hexanes) to afford (Z)-6-methyl-3,5-diphenylbenzo[ $[g][1,2,3]$ triazolo[1,5-a $][1,5]$ diazocin-7(6H)-one 3a as yellow solid (400 $\mathrm{mg}, 80 \%)$.

\section{Characterization data of compounds $2 b-2 n$ and $3 b-3 k$}


(Z)-4-benzylidene-5-ethyl-9-fluoro-3-(p-tolyl)-4,5-dihydro-6H-benzo[f] $[1,2,3]$ tri azolo[1,5-a][1,4]diazepin-6-one $(2 \mathrm{~b})$

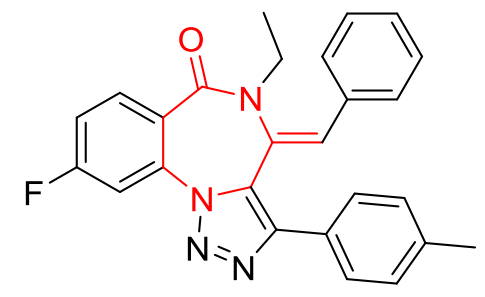

Off white solid (80 mg, 80\%), (eluent: $8-10 \%$ ethyl acetate in hexanes); mp 172-174 ${ }^{\circ} \mathrm{C} ;{ }^{1} \mathrm{H}$ NMR (400 $\left.\mathrm{MHz}, \mathrm{CDCl}_{3}\right) \delta 8.18(\mathrm{dd}, J=8.8,5.9 \mathrm{~Hz}, 1 \mathrm{H}), 7.96(\mathrm{~d}$, $J=8.2 \mathrm{~Hz}, 2 \mathrm{H}), 7.78(\mathrm{dd}, J=9.1,2.5 \mathrm{~Hz}, 1 \mathrm{H}), 7.43-$ $7.35(\mathrm{~m}, 5 \mathrm{H}), 7.31(\mathrm{~d}, J=8.0 \mathrm{~Hz}, 2 \mathrm{H}), 7.24-7.22(\mathrm{~m}, 2 \mathrm{H}), 6.70(\mathrm{~s}, 1 \mathrm{H}), 4.41(\mathrm{dd}, J$ $=13.7,7.1 \mathrm{~Hz}, 1 \mathrm{H}), 3.15(\mathrm{dd}, J=13.7,7.1 \mathrm{~Hz}, 1 \mathrm{H}), 2.42(\mathrm{~s}, 3 \mathrm{H}), 1.12(\mathrm{t}, J=7.2 \mathrm{~Hz}$ $3 \mathrm{H}) ;{ }^{13} \mathrm{C} \mathrm{NMR}\left(101 \mathrm{MHz}, \mathrm{CDCl}_{3}\right) \delta 164.6,164.5\left(\mathrm{~d},{ }^{1} \mathrm{~J}=254.5 \mathrm{~Hz}\right), 141.7,138.7$, $134.9,134.4,134.3,134.1,132.2,130.0,129.7,129.1,129.0,126.8,126.3,123.7$, 122.9, $116.2\left(\mathrm{~d},{ }^{2} J=21.6 \mathrm{~Hz}\right), 109.7\left(\mathrm{~d},{ }^{2} J=26.8 \mathrm{~Hz}\right), 44.9,21.3,14.2$; HRMS (ESI) calcd for $\mathrm{C}_{26} \mathrm{H}_{21} \mathrm{FN}_{4} \mathrm{O} m / z$ : 425.1772; Found $425.1798(\mathrm{M}+\mathrm{H})^{+}$.

(Z)-4-benzylidene-5-isopropyl-3-phenyl-4,5-dihydro-6H-benzo[f][1,2,3]triazolo[ 1,5-a][1,4]diazepin-6-one (2c)

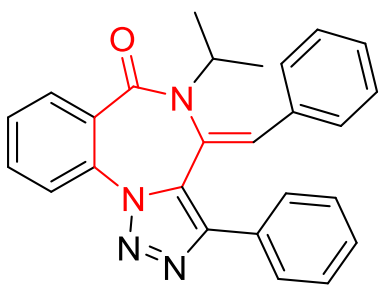

White solid (78 mg, $78 \%$ ), (eluent: 8-10\% ethyl acetate in hexanes); mp 113-115 ${ }^{\circ} \mathrm{C} ;{ }^{1} \mathrm{H}$ NMR (400 $\left.\mathrm{MHz}, \mathrm{CDCl}_{3}\right) \delta$ $8.13-8.10(\mathrm{~m}, 3 \mathrm{H}), 8.00(\mathrm{~d}, J=8.1 \mathrm{~Hz}, 1 \mathrm{H}), 7.66(\mathrm{t}, J=$ $7.7 \mathrm{~Hz}, 1 \mathrm{H}), 7.57-7.50(\mathrm{~m}, 3 \mathrm{H}), 7.47-7.37(\mathrm{~m}, 6 \mathrm{H}), 6.95$ $(\mathrm{s}, 1 \mathrm{H}), 4.56(\mathrm{sept}, J=6.8 \mathrm{~Hz}, 1 \mathrm{H}), 1.03(\mathrm{t}, J=5.8 \mathrm{~Hz}, 6 \mathrm{H}) ;{ }^{13} \mathrm{C} \mathrm{NMR}(101 \mathrm{MHz}$, $\left.\mathrm{CDCl}_{3}\right) \delta 166.4,141.1,138.3,135.2,133.2,133.1,132.4,131.3,130.1,129.9,129.2$, 128.9, 128.9, 128.9, 128.6, 128.2, 53.1, 21.6, 19.8; HRMS (ESI) calcd for $\mathrm{C}_{26} \mathrm{H}_{22} \mathrm{~N}_{4} \mathrm{O} m / z:$ 407.1866; Found 407.1863 (M+H) .

(Z)-4-benzylidene-5-ethyl-3-phenyl-4,5-dihydro-6H-benzo[f][1,2,3]triazolo[1,5-a 


\section{][1,4]diazepin-6-one (2d)}

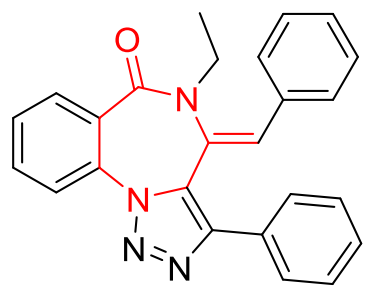

Pale yellow solid (80 mg, $80 \%$ ), (eluent: $8-10 \%$ ethyl acetate in hexanes); mp $109-111{ }^{\circ} \mathrm{C} ;{ }^{1} \mathrm{H}$ NMR (400 MHz, $\left.\mathrm{CDCl}_{3}\right) \delta$ $8.15(\mathrm{~d}, J=7.8 \mathrm{~Hz}, 1 \mathrm{H}), 8.07(\mathrm{t}, J=8.1 \mathrm{~Hz}, 3 \mathrm{H}), 7.68(\mathrm{t}, J=$ $7.7 \mathrm{~Hz}, 1 \mathrm{H}), 7.60-7.5(\mathrm{~m}, 3 \mathrm{H}), 7.43-7.35(\mathrm{~m}, 6 \mathrm{H}), 6.69(\mathrm{~s}$, $1 \mathrm{H}), 4.41(\mathrm{q}, J=13.12 \mathrm{~Hz}, 1 \mathrm{H}), 3.16(\mathrm{q}, J=13.7 \mathrm{~Hz}, 1 \mathrm{H}), 1.12(\mathrm{t}, J=7.1 \mathrm{~Hz}, 3 \mathrm{H})$;

${ }^{13} \mathrm{C}$ NMR $\left(101 \mathrm{MHz}, \mathrm{CDCl}_{3}\right) \delta 165.4,141.4,134.6,134.0,133.4,132.7,132.3$, $131.7,129.9,129.1,129.0,128.9,128.9,128.6,126.8,126.4,124.0,122.5,44.9$, 14.1; HRMS (ESI) calcd for $\mathrm{C}_{25} \mathrm{H}_{20} \mathrm{~N}_{4} \mathrm{O}$ m/z: 393.1710; Found 393.1708 (M+H) ${ }^{+}$.

(Z)-4-benzylidene-5-cycloheptyl-9-fluoro-3-phenyl-4,5-dihydro-6H-benzo[f] $[1,2$, 3]triazolo[1,5-a][1,4]diazepin-6-one (2e)

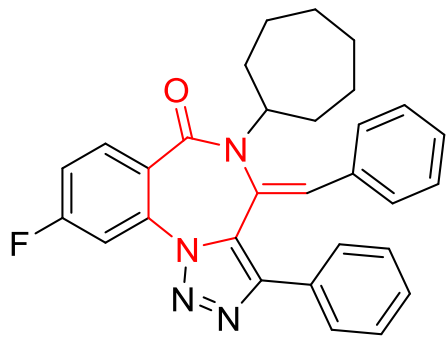

White solid (74 mg, $74 \%$ ), (eluent: 8-10\% ethyl acetate in hexanes); mp 201-203 ${ }^{\circ} \mathrm{C} ;{ }^{1} \mathrm{H}$ NMR $\left(400 \mathrm{MHz}, \mathrm{CDCl}_{3}\right)$ $\delta 8.12-8.06(\mathrm{~m}, 3 \mathrm{H}), 7.73(\mathrm{dd}, J=9.0,2.5 \mathrm{~Hz}, 1 \mathrm{H})$, $7.54-7.50(\mathrm{~m}, 2 \mathrm{H}), 7.47-7.37(\mathrm{~m}, 6 \mathrm{H}), 7.26-7.22(\mathrm{~m}$,

1H), $6.83(\mathrm{~s}, 1 \mathrm{H}), 3.97(\mathrm{t}, J=10.4 \mathrm{~Hz}, 1 \mathrm{H}), 1.88-1.66(\mathrm{~m}, 5 \mathrm{H}), 1.45-1.25(\mathrm{~m}$, $7 \mathrm{H}) ;{ }^{13} \mathrm{C} \mathrm{NMR}\left(101 \mathrm{MHz}, \mathrm{CDCl}_{3}\right) \delta 165.3,164.3\left(\mathrm{~d},{ }^{1} \mathrm{~J}=254.1 \mathrm{~Hz}\right), 141.6,137.2$, $134.8,134.5,134.4,133.9,133.8,132.8,129.9,129.8,129.0,128.9,128.9,128.7$, $116.4\left(\mathrm{~d},{ }^{2} J=21.7 \mathrm{~Hz}\right), 109.5\left({ }^{2} J=26.6 \mathrm{~Hz}\right), 65.8,34.2,32.0,27.6,26.5,25.2,25.1$; HRMS (ESI) calcd for $\mathrm{C}_{30} \mathrm{H}_{27} \mathrm{FN}_{4} \mathrm{O} m / z$ : 479.2242; Found $479.2248(\mathrm{M}+\mathrm{H})^{+}$. 


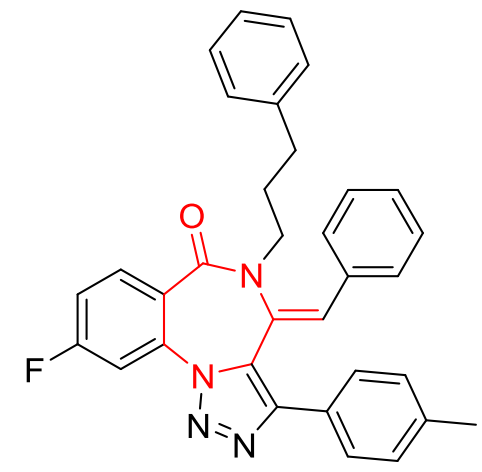

Off white solid (82 mg, $82 \%$ ), (eluent: $8-10 \%$ ethyl acetate in hexanes); mp 192-194 ${ }^{\circ} \mathrm{C} ;{ }^{1} \mathrm{H}$ NMR (400 $\left.\mathrm{MHz}, \mathrm{CDCl}_{3}\right) \delta 8.19(\mathrm{dd}, J=8.8,5.9 \mathrm{~Hz}, 1 \mathrm{H}), 7.91(\mathrm{~d}$, $J=8.2 \mathrm{~Hz}, 2 \mathrm{H}), 7.79(\mathrm{dd}, J=9.1,2.5 \mathrm{~Hz}, 1 \mathrm{H}), 7.42-$ $7.35(\mathrm{~m}, 5 \mathrm{H}), 7.29-7.22(\mathrm{~m}, 3 \mathrm{H}), 7.15-7.10(\mathrm{~m}, 3 \mathrm{H})$, $6.85(\mathrm{~d}, J=7.7 \mathrm{~Hz}, 2 \mathrm{H}), 6.72(\mathrm{~s}, 1 \mathrm{H}), 4.44-4.36(\mathrm{~m}$, $J=1 \mathrm{H}), 3.09-3.02(\mathrm{~m}, 1 \mathrm{H}), 2.44(\mathrm{~s}, 3 \mathrm{H}), 2.40(\mathrm{~d}, J=8.7 \mathrm{~Hz}, 1 \mathrm{H}), 1.98-1.87(\mathrm{~m}$, 1H), $1.73-1.62(\mathrm{~m}, 1 \mathrm{H}) ;{ }^{13} \mathrm{C} \mathrm{NMR}\left(101 \mathrm{MHz}, \mathrm{CDCl}_{3}\right) \delta 164.7,164.6\left(\mathrm{~d},{ }^{1} J=254.7\right.$ Hz), 141.8, 140.5, 138.7, 135.0, 134.9, 134.6, 134.5, 134.4, 134.01 , 132.2, 130.0, $129.7,129.1,129.0,128.2,128.0,126.7,126.4,125.8,123.8,122.8,122.8,116.2(d$, $\left.{ }^{2} J=21.6 \mathrm{~Hz}\right), 109.8\left(\mathrm{~d},{ }^{2} J=26.8 \mathrm{~Hz}\right), 49.2,33.1,30.3,21.3$; HRMS (ESI) calcd for $\mathrm{C}_{33} \mathrm{H}_{27} \mathrm{FN}_{4} \mathrm{O} m / z: 515.2242$; Found $515.2240(\mathrm{M}+\mathrm{H})^{+}$.

(Z)-4-benzylidene-3-(4-methoxyphenyl)-5-(3-phenylpropyl)-4,5-dihydro-6H-ben zo[f][1,2,3]triazolo[1,5-a][1,4]diazepin-6-one (2g)

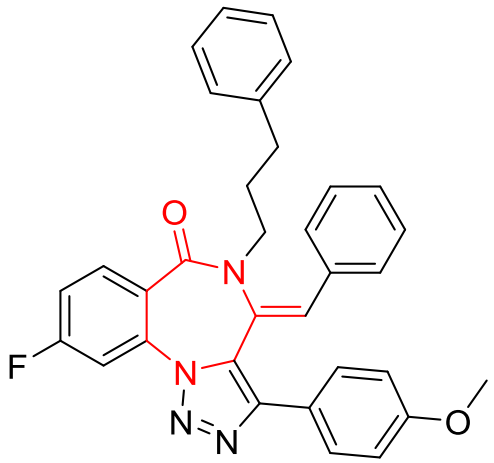

White solid (78 mg, $78 \%$ ), (eluent: $8-10 \%$ ethyl acetate in hexanes); mp $183-185{ }^{\circ} \mathrm{C} ;{ }^{1} \mathrm{H}$ NMR (400 $\left.\mathrm{MHz}, \mathrm{CDCl}_{3}\right) \delta 8.15(\mathrm{~d}, J=7.6 \mathrm{~Hz}, 1 \mathrm{H}), 8.05(\mathrm{~d}, J$ $=8.0 \mathrm{~Hz}, 1 \mathrm{H}), 7.94(\mathrm{~d}, J=8.6 \mathrm{~Hz}, 2 \mathrm{H}), 7.68(\mathrm{t}, J=$ $7.6 \mathrm{~Hz}, 1 \mathrm{H}), 7.55(\mathrm{t}, J=7.5 \mathrm{~Hz}, 1 \mathrm{H}), 7.39-7.35(\mathrm{~m}$, $5 \mathrm{H}), 7.15-7.09(\mathrm{~m}, 3 \mathrm{H}), 6.98(\mathrm{~d}, J=8.6 \mathrm{~Hz}, 2 \mathrm{H})$, $6.85(\mathrm{~d}, J=6.7 \mathrm{~Hz}, 2 \mathrm{H}), 6.70(\mathrm{~s}, 1 \mathrm{H}), 4.40(\mathrm{t}, J=14.7 \mathrm{~Hz}, 1 \mathrm{H}), 3.88(\mathrm{~s}, 3 \mathrm{H}), 3.09-$ $3.02(\mathrm{~m}, 1 \mathrm{H}), 2.49-2.35(\mathrm{~m}, 2 \mathrm{H}), 2.03-1.83(\mathrm{~m}, 1 \mathrm{H}), 1.77-1.53(\mathrm{~m}, 1 \mathrm{H}) ;{ }^{13} \mathrm{C}$ NMR $\left(101 \mathrm{MHz}, \mathrm{CDCl}_{3}\right) \delta 165.6,159.8,141.5,140.6,134.1,133.7,133.5,132.7$, $132.3,131.8,129.9,129.1,129.0,128.8,128.2,128.1,127.9,126.7,125.8,124.3$, 
122.5, 122.4, 114.3, 55.3, 49.1, 33.1, 30.3; HRMS (ESI) calcd for $\mathrm{C}_{33} \mathrm{H}_{28} \mathrm{~N}_{4} \mathrm{O}_{2} m / z$ : 513.2285; Found $513.2287(\mathrm{M}+\mathrm{H})^{+}$.

(Z)-9-chloro-3-cyclohexyl-5-(3-methoxypropyl)-4-(4-(trifluoromethyl)benzylide ne)-4,5-dihydro-6H-benzo[f][1,2,3]triazolo[1,5-a][1,4]diazepin-6-one (2h)

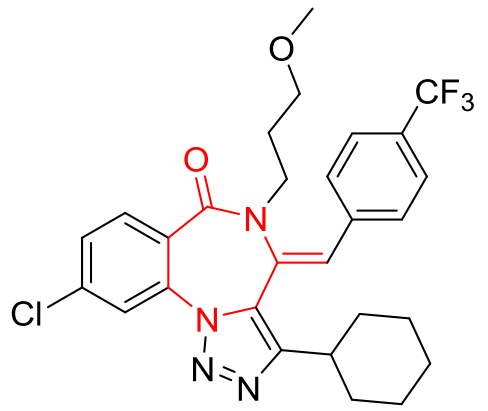

Off white solid (81 mg, $81 \%)$, (eluent: $8-10 \%$ ethyl acetate in hexanes); mp 200-202 ${ }^{\circ} \mathrm{C} ;{ }^{1} \mathrm{H}$ NMR (400 $\left.\mathrm{MHz}, \mathrm{CDCl}_{3}\right) \delta 8.05(\mathrm{dd}, J=8.5,1.2 \mathrm{~Hz}, 1 \mathrm{H}), 8.01(\mathrm{t}$, $J=2.0 \mathrm{~Hz}, 1 \mathrm{H}), 7.65(\mathrm{~d}, J=7.8 \mathrm{~Hz}, 2 \mathrm{H}), 7.51-7.45$ $(\mathrm{m}, 3 \mathrm{H}), 6.51(\mathrm{~s}, 1 \mathrm{H}), 4.36(\mathrm{dt}, J=13.9,7.4 \mathrm{~Hz}, 1 \mathrm{H})$ $3.23-3.17(\mathrm{~m}, 2 \mathrm{H}), 3.12(\mathrm{~s}, 3 \mathrm{H}), 3.03-2.96(\mathrm{~m}, 1 \mathrm{H}), 2.81-2.77(\mathrm{~m}, 1 \mathrm{H}), 1.96-$ $1.72(\mathrm{~m}, 10 \mathrm{H}), 1.67-1.57(\mathrm{~m}, 2 \mathrm{H}) ;{ }^{13} \mathrm{C} \mathrm{NMR}\left(101 \mathrm{MHz}, \mathrm{CDCl}_{3}\right) \delta$ 164.7, 148.2, 139.0, 135.7, 134.3, 133.4, $131.4\left(\mathrm{q},{ }^{2} J=33.0 \mathrm{~Hz}\right), 129.2,128.8,126.4,126.0\left(\mathrm{q},{ }^{3} J\right.$ $=3.6 \mathrm{~Hz}), 124.4,122.4,69.7,58.4,47.1,35.0,33.5,32.3,28.4,26.5,26.3,25.7$; HRMS (ESI) calcd for $\mathrm{C}_{28} \mathrm{H}_{28} \mathrm{ClF}_{3} \mathrm{~N}_{4} \mathrm{O}_{2}$ m/z: 545.1926; Found 545.1930 (M+H) ${ }^{+}$.

(Z)-9-chloro-5-ethyl-4-hexylidene-3-pentyl-4,5-dihydro-6H-benzo[f][1,2,3]triazo lo[1,5-a][1,4]diazepin-6-one (2i)

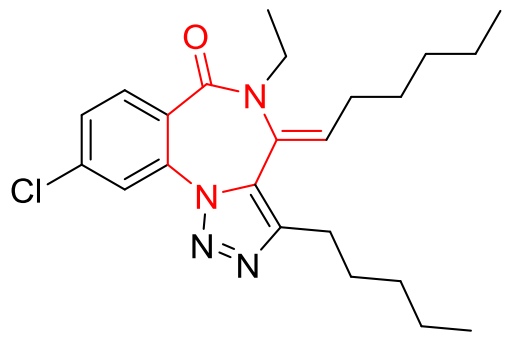

Pale yellow solid (81 mg, $81 \%$ ), (eluent: $8-10 \%$ ethyl acetate in hexanes); mp 211-213 ${ }^{\circ} \mathrm{C} ;{ }^{1} \mathrm{H}$ NMR $\left(400 \mathrm{MHz}, \mathrm{CDCl}_{3}\right) \delta 7.49(\mathrm{~s}, 2 \mathrm{H}), 7.45(\mathrm{~s}, 1 \mathrm{H}), 6.10$ $(\mathrm{s}, 1 \mathrm{H}), 4.19-4.10(\mathrm{~m}, 1 \mathrm{H}), 3.12-3.03(\mathrm{~m}, 1 \mathrm{H})$ $2.73-2.60(\mathrm{~m}, 2 \mathrm{H}), 2.45-2.38(\mathrm{~m}, 1 \mathrm{H}), 2.27-2.19(\mathrm{~m}, 1 \mathrm{H}), 1.74-1.71(\mathrm{~m}, 2 \mathrm{H})$, $1.41-1.31(\mathrm{~m}, 4 \mathrm{H}), 1.28-1.04(\mathrm{~m}, 6 \mathrm{H}), 0.95(\mathrm{t}, J=7.1 \mathrm{~Hz}, 3 \mathrm{H}), 0.91(\mathrm{t}, J=6.4 \mathrm{~Hz}$ 3H), $0.80(\mathrm{t}, J=7.2 \mathrm{~Hz}, 3 \mathrm{H}) ;{ }^{13} \mathrm{C} \mathrm{NMR}\left(101 \mathrm{MHz}, \mathrm{CDCl}_{3}\right) \delta 165.2,148.6,144.9$, $136.4,133.6,131.3,130.1,129.8,129.4,125.9,112.6,39.7,34.0,31.5,30.9,28.5$, 26.6, 25.4, 22.3, 22.3, 13.9, 13.8, 12.6; HRMS (ESI) calcd for $\mathrm{C}_{23} \mathrm{H}_{31} \mathrm{ClN}_{4} \mathrm{O} m / z$ : 
415.2259; Found 415.2263 $(\mathrm{M}+\mathrm{H})^{+}$.

(Z)-4-benzylidene-9-chloro-3-pentyl-5-(3-phenylpropyl)-4,5-dihydro-6H-benzo[ f][1,2,3] triazolo[1,5-a][1,4]diazepin-6-one $(2 \mathrm{j})$

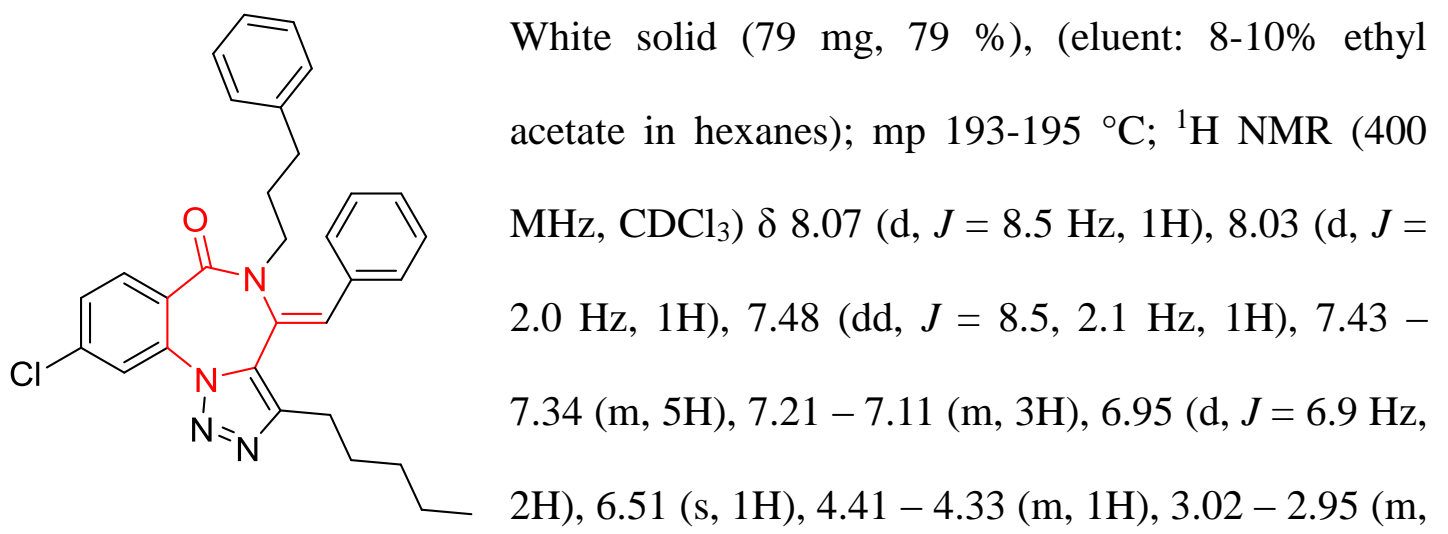

$1 \mathrm{H}), 2.83-2.70(\mathrm{~m}, 2 \mathrm{H}), 2.42-2.37(\mathrm{~m}, 2 \mathrm{H}), 1.91-1.78(\mathrm{~m}, 3 \mathrm{H}), 1.72-1.62(\mathrm{~m}$, $1 \mathrm{H}), 1.47-1.33(\mathrm{~m}, 4 \mathrm{H}), 0.93(\mathrm{t}, J=7.1 \mathrm{~Hz}, 3 \mathrm{H}) ;{ }^{13} \mathrm{C} \mathrm{NMR}\left(101 \mathrm{MHz}, \mathrm{CDCl}_{3}\right) \delta$ $164.8,143.5,140.6,138.8,135.7,134.4,133.5,133.0,132.3,129.8,129.1,128.9$, $128.7,128.3,128.0,125.9,124.8,123.3,122.3,48.3,33.0,31.8,30.2,28.9,25.0$, 22.4, 14.0; HRMS (ESI) calcd for $\mathrm{C}_{31} \mathrm{H}_{31} \mathrm{ClN}_{4} \mathrm{O} m / z$ : 511.2259; Found 511.2252 $(\mathrm{M}+\mathrm{H})^{+}$

(Z)-4-butylidene-5-(4-methoxybenzyl)-9-methyl-3-phenyl-4,5-dihydro-6H-benz $o[f][1,2,3]$ triazolo[1,5-a][1,4]diazepin-6-one (2k)

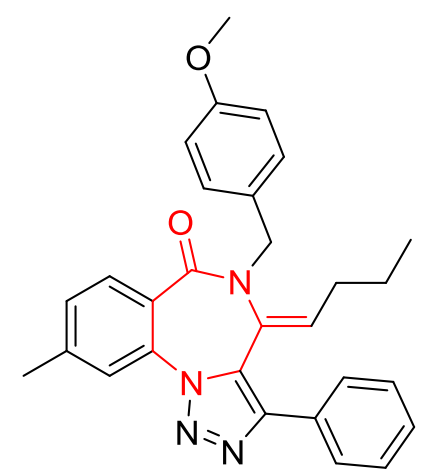

White solid (74 mg, $74 \%$ ), (eluent: 8-10\% ethyl acetate in hexanes); mp 131-133 ${ }^{\circ} \mathrm{C} ;{ }^{1} \mathrm{H}$ NMR $\left(400 \mathrm{MHz}, \mathrm{CDCl}_{3}\right) \delta$ $7.54(\mathrm{~d}, J=7.9 \mathrm{~Hz}, 1 \mathrm{H}), 7.37(\mathrm{~d}, J=7.9 \mathrm{~Hz}, 1 \mathrm{H}), 7.32(\mathrm{dd}$, $J=6.5,2.8 \mathrm{~Hz}, 2 \mathrm{H}), 7.25-7.23(\mathrm{~m}, 3 \mathrm{H}), 6.83(\mathrm{~d}, J=8.5$ $\mathrm{Hz}, 2 \mathrm{H}), 6.39$ (d, $J=8.4 \mathrm{~Hz}, 2 \mathrm{H}), 6.05(\mathrm{~s}, 1 \mathrm{H}), 5.68(\mathrm{~d}, J=$ $14.7 \mathrm{~Hz}, 1 \mathrm{H}), 3.92(\mathrm{~d}, J=14.7 \mathrm{~Hz}, 1 \mathrm{H}), 3.34(\mathrm{~s}, 3 \mathrm{H})$, $2.61-2.55(\mathrm{~m}, 1 \mathrm{H}), 2.42(\mathrm{~s}, 3 \mathrm{H}), 2.27-2.19(\mathrm{~m}, 1 \mathrm{H}), 1.82(\mathrm{~s}, 1 \mathrm{H}), 1.61-1.49(\mathrm{~m}$, 1H), $1.33-1.20(\mathrm{~m}, 1 \mathrm{H}), 0.75(\mathrm{t}, J=7.3 \mathrm{~Hz}, 3 \mathrm{H}) ;{ }^{13} \mathrm{C} \mathrm{NMR}\left(101 \mathrm{MHz}, \mathrm{CDCl}_{3}\right) \delta$ 
$166.7,159.0,148.1,143.1,141.6,132.3,130.6,130.1,129.6,129.2,129.0,128.9$, $128.2,127.7,127.1,126.3,126.1,114.4,113.9,54.7,47.2,36.0,21.1,20.0,13.4$; HRMS (ESI) calcd for $\mathrm{C}_{29} \mathrm{H}_{28} \mathrm{~N}_{4} \mathrm{O}_{2} \mathrm{~m} / z:$ 465.2285; Found $465.2283(\mathrm{M}+\mathrm{H})^{+}$.

(Z)-4-butylidene-9-chloro-5-(4-methoxybenzyl)-3-(4-(trifluoromethyl)phenyl)-4, 5-dihydro-6H-benzo[f][1,2,3]triazolo[1,5-a][1,4]diazepin-6-one (2l)

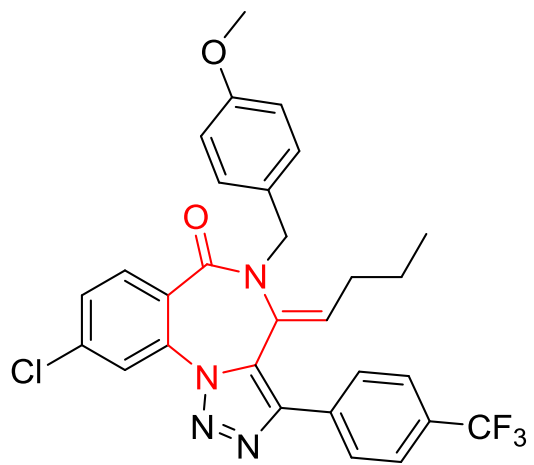

Off white solid (77 mg, $77 \%$ ), (eluent: 8-10\% ethyl acetate in hexanes); mp 159-161 ${ }^{\circ} \mathrm{C} ;{ }^{1} \mathrm{H}$ NMR (400 $\left.\mathrm{MHz}, \mathrm{CDCl}_{3}\right) \delta 7.63-7.55(\mathrm{~m}, 2 \mathrm{H}), 7.53-7.48(\mathrm{~m}$, $3 \mathrm{H}), 7.40(\mathrm{~d}, J=8.2 \mathrm{~Hz}, 2 \mathrm{H}), 6.82(\mathrm{~d}, J=8.5 \mathrm{~Hz}$, 2H), $6.39(\mathrm{~d}, J=8.6 \mathrm{~Hz}, 2 \mathrm{H}), 6.06(\mathrm{~s}, 1 \mathrm{H}), 5.74(\mathrm{~d}$, $J=14.7 \mathrm{~Hz}, 1 \mathrm{H}), 3.91(\mathrm{~d}, J=14.7 \mathrm{~Hz}, 1 \mathrm{H}), 3.34(\mathrm{~s}$, $3 \mathrm{H}), 2.69-2.57(\mathrm{~m}, 1 \mathrm{H}), 2.35-2.23(\mathrm{~m}, 1 \mathrm{H}), 1.38-1.20(\mathrm{~m}, 2 \mathrm{H}), 0.88(\mathrm{t}, J=7.0$ $\mathrm{Hz}, 1 \mathrm{H}), 0.82(\mathrm{t}, J=7.3 \mathrm{~Hz}, 3 \mathrm{H}) ;{ }^{13} \mathrm{C} \mathrm{NMR}\left(101 \mathrm{MHz}, \mathrm{CDCl}_{3}\right) \delta 165.3,159.2$, $148.9,142.0,136.8,133.2,132.9,130.9,130.4,129.6,129.4,126.7,126.1,126.0$, $125.2\left(\mathrm{q},{ }^{3} J=3.7 \mathrm{~Hz}\right), 113.9,113.9,54.5,47.1,35.9,20.1,13.4$; HRMS (ESI) calcd for $\mathrm{C}_{29} \mathrm{H}_{24} \mathrm{ClF}_{3} \mathrm{~N}_{4} \mathrm{O}_{2} \mathrm{~m} / z$ : 553.1613; Found $553.1625(\mathrm{M}+\mathrm{H})^{+}$.

(Z)-4-(cyclohexylmethylene)-5-cyclopropyl-9-methyl-3-propyl-4,5-dihydro-6Hbenzo[f][1,2,3]triazolo[1,5-a][1,4]diazepin-6-one $(2 \mathrm{~m})$

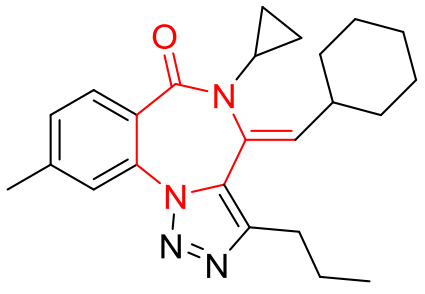

White solid (82 mg, $82 \%$ ), (eluent: $8-10 \%$ ethyl acetate in hexanes); mp 166-168 ${ }^{\circ} \mathrm{C} ;{ }^{1} \mathrm{H}$ NMR $\left(400 \mathrm{MHz}, \mathrm{CDCl}_{3}\right)$ $\delta 7.53(\mathrm{~d}, J=7.9 \mathrm{~Hz}, 1 \mathrm{H}), 7.30(\mathrm{~d}, J=7.8 \mathrm{~Hz}, 1 \mathrm{H}), 7.15$ (s, 1H), $5.95(\mathrm{~s}, 1 \mathrm{H}), 2.83-2.78(\mathrm{~m}, 1 \mathrm{H}), 2.67-2.54(\mathrm{~m}$, 2H), $2.39(\mathrm{~s}, 3 \mathrm{H}), 1.93-1.66(\mathrm{~m}, 7 \mathrm{H}), 1.35-1.18(\mathrm{~m}, 4 \mathrm{H}), 1.06(\mathrm{~d}, J=12.7 \mathrm{~Hz}$, $1 \mathrm{H}), 0.99(\mathrm{t}, J=7.3 \mathrm{~Hz}, 3 \mathrm{H}), 0.95-0.87(\mathrm{~m}, 1 \mathrm{H}), 0.81-0.62(\mathrm{~m}, 2 \mathrm{H}), 0.37-0.30$ (m, 1H), $0.13-0.07(\mathrm{~m}, 1 \mathrm{H}) ;{ }^{13} \mathrm{C}$ NMR $\left(101 \mathrm{MHz}, \mathrm{CDCl}_{3}\right) \delta$ 169.0, 154.4, 141.7, 
$130.3,130.0,129.7,126.2,109.0,41.7,31.8,29.8,28.3,27.3,26.4,25.9,25.8,22.2$, 21.1, 14.0, 9.7, 5.4; HRMS (ESI) calcd for $\mathrm{C}_{24} \mathrm{H}_{30} \mathrm{~N}_{4} \mathrm{O} \mathrm{m} / z$ : 391.2492; Found $391.2501(\mathrm{M}+\mathrm{H})^{+}$.

(Z)-5-(benzo[d][1,3]dioxol-5-ylmethyl)-4-benzylidene-3-(4-(trifluoromethyl)phe nyl)-4,5-dihydro-6H-naphtho[2,3-f][1,2,3]triazolo[1,5-a][1,4]diazepin-6-one (2n)

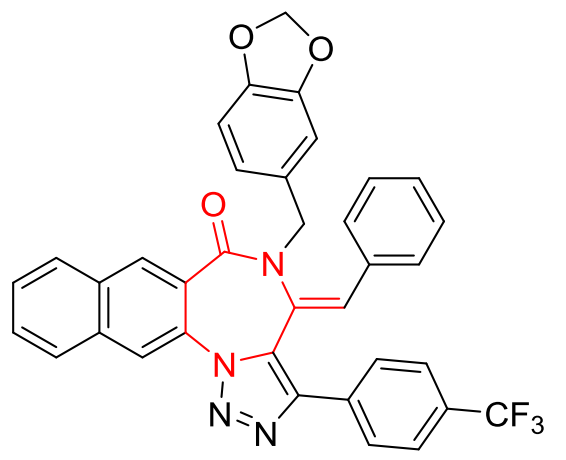

White solid (84 mg, $84 \%$ ), (eluent: 8-10\% ethyl acetate in hexanes); mp $181-183{ }^{\circ} \mathrm{C} ;{ }^{1} \mathrm{H}$ NMR $\left(400 \mathrm{MHz}, \mathrm{CDCl}_{3}\right) \delta 8.82(\mathrm{~s}, 1 \mathrm{H}), 8.50(\mathrm{~s}, 1 \mathrm{H})$, $8.06(\mathrm{~d}, J=7.8 \mathrm{~Hz}, 1 \mathrm{H}), 7.99(\mathrm{~d}, J=7.8 \mathrm{~Hz}, 1 \mathrm{H})$, $7.73-7.61(\mathrm{~m}, 4 \mathrm{H}), 7.57(\mathrm{~d}, J=8.3 \mathrm{~Hz}, 2 \mathrm{H})$, $7.49-7.38(\mathrm{~m}, 5 \mathrm{H}), 6.74(\mathrm{~s}, 1 \mathrm{H}), 6.39-6.36(\mathrm{~m}$, 2H), $6.32(\mathrm{~d}, J=8.0 \mathrm{~Hz}, 1 \mathrm{H}), 5.80(\mathrm{~d}, J=14.7 \mathrm{~Hz}, 1 \mathrm{H}), 5.74(\mathrm{~d}, J=1.3 \mathrm{~Hz}, 1 \mathrm{H})$, $5.62(\mathrm{~d}, J=1.2 \mathrm{~Hz}, 1 \mathrm{H}), 3.98(\mathrm{~d}, J=14.7 \mathrm{~Hz}, 1 \mathrm{H}) ;{ }^{13} \mathrm{C} \mathrm{NMR}\left(101 \mathrm{MHz}, \mathrm{CDCl}_{3}\right) \delta$ $166.2,147.9,147.3,140.3,135.3,135.2,134.5,133.8,133.2,132.2,132.2,130.2$ 130.0, 129.3, 129.0, 129.0, 128.8, 128.1, 127.9, 126.4, 125.3 (q, $\left.{ }^{3} J=4.3 \mathrm{~Hz}\right), 124.6$, 123.4, 121.7, 121.4, 108.3, 108.1, 100.9, 49.6; HRMS (ESI) calcd for $\mathrm{C}_{36} \mathrm{H}_{23} \mathrm{~F}_{3} \mathrm{~N}_{4} \mathrm{O}_{3}$ $m / z:$ 617.1795; Found 617.1773 $(\mathrm{M}+\mathrm{H})^{+}$.

\section{(Z)-6-ethyl-10-fluoro-5-phenyl-3-(p-tolyl)benzo[g][1,2,3]triazolo[1,5-a][1,5]diaz} ocin-7(6H)-one (3b)<smiles>CCN1C(=O)c2ccc(F)cc2-n2nnc(-c3ccc(C)cc3)c2/C=C\1c1ccccc1</smiles>
Pale yellow solid (83 mg, $83 \%$ ), (eluent: $8-10 \%$ ethyl acetate in hexanes); mp 180-182 ${ }^{\circ} \mathrm{C} ;{ }^{1} \mathrm{H}$ NMR (400 MHz, $\left.\mathrm{CDCl}_{3}\right) \delta 8.18(\mathrm{dd}, J=8.8,5.9 \mathrm{~Hz}, 1 \mathrm{H}), 7.96(\mathrm{~d}, J=8.2$ $\mathrm{Hz}, 2 \mathrm{H}), 7.78(\mathrm{dd}, J=9.1,2.5 \mathrm{~Hz}, 1 \mathrm{H}), 7.46-7.34(\mathrm{~m}$,

$7 \mathrm{H}), 7.31(\mathrm{~d}, J=8.0 \mathrm{~Hz}, 2 \mathrm{H}), 7.27-7.20(\mathrm{~m}, 1 \mathrm{H}), 6.70(\mathrm{~s}, 1 \mathrm{H}), 4.41(\mathrm{dq}, J=14.3$, $7.2 \mathrm{~Hz}, 1 \mathrm{H}), 3.15(\mathrm{dq}, J=14.2,7.1 \mathrm{~Hz}, 1 \mathrm{H}), 2.42(\mathrm{~s}, 3 \mathrm{H}), 1.12(\mathrm{t}, J=7.2 \mathrm{~Hz}, 3 \mathrm{H})$; 
${ }^{13} \mathrm{C} \mathrm{NMR}\left(101 \mathrm{MHz}, \mathrm{CDCl}_{3}\right) \delta 165.9,163.2\left(\mathrm{~d},{ }^{1} J=252.5 \mathrm{~Hz}\right), 147.3,143.6,138.5$, $137.8,134.3,130.8,130.7,130.4,129.7,129.4,129.2,128.7,128.6,126.3,126.2$, $117.6\left(\mathrm{~d},{ }^{2} J=20.2 \mathrm{~Hz}\right), 113.4\left(\mathrm{~d},{ }^{2} J=20.2 \mathrm{~Hz}\right), 113.2,41.8,21.3,13.0$; HRMS (ESI) calcd for $\mathrm{C}_{26} \mathrm{H}_{21} \mathrm{FN}_{4} \mathrm{O} m / z$ : 425.1772 ; Found $425.1785(\mathrm{M}+\mathrm{H})^{+}$.

\section{(Z)-6-isopropyl-3,5-diphenylbenzo[g][1,2,3]triazolo[1,5-a][1,5]diazocin-7(6H)-o} ne (3c)<smiles>CC(C)N1C(=O)c2ccccc2-n2nnc(-c3ccccc3)c2C=C1c1ccccc1</smiles>
Yellow solid (83 mg, $83 \%$ ), (eluent: 8-10\% ethyl acetate in hexanes); mp 111-113 ${ }^{\circ} \mathrm{C} ;{ }^{1} \mathrm{H}$ NMR (400 MHz, $\left.\mathrm{CDCl}_{3}\right) \delta 8.06$ $(\mathrm{d}, J=8.3 \mathrm{~Hz}, 2 \mathrm{H}), 7.80-7.75(\mathrm{~m}, 1 \mathrm{H}), 7.61-7.50(\mathrm{~m}, 6 \mathrm{H})$, $7.50-7.39(\mathrm{~m}, 5 \mathrm{H}), 6.70(\mathrm{~s}, 1 \mathrm{H}), 4.52-4.42(\mathrm{~m}, 1 \mathrm{H}), 1.07(\mathrm{~d}$, $J=6.9 \mathrm{~Hz}, 3 \mathrm{H}), 0.97(\mathrm{~d}, J=6.8 \mathrm{~Hz}, 3 \mathrm{H}) ;{ }^{13} \mathrm{C} \mathrm{NMR}\left(101 \mathrm{MHz}, \mathrm{CDCl}_{3}\right) \delta 166.5$, $147.7,143.1,137.1,133.2,132.4,130.8,130.5,130.2,130.0,129.0,128.9,128.6$, $128.3,126.7,126.2,125.8,115.6,50.4,21.0,20.5$; HRMS (ESI) calcd for $\mathrm{C}_{26} \mathrm{H}_{22} \mathrm{~N}_{4} \mathrm{O} m / z: 407.1866$; Found $407.1873(\mathrm{M}+\mathrm{H})^{+}$.

\section{(Z)-6-ethyl-3,5-diphenylbenzo[g][1,2,3]triazolo[1,5-a][1,5]diazocin-7(6H)-one}

(3d)<smiles>CCN1C(=O)c2ccccc2-n2nnc(-c3ccccc3)c2C=C1c1ccccc1</smiles>

Yellow solid (82 mg, $82 \%$ ), (eluent: $8-10 \%$ ethyl acetate in hexanes); mp 106-108 ${ }^{\circ} \mathrm{C} ;{ }^{1} \mathrm{H}$ NMR $\left(400 \mathrm{MHz}, \mathrm{CDCl}_{3}\right) \delta 8.04$ $(\mathrm{d}, J=7.2 \mathrm{~Hz}, 2 \mathrm{H}), 7.75-7.71(\mathrm{~m}, 1 \mathrm{H}), 7.59-7.39(\mathrm{~m}, 11 \mathrm{H})$, $6.77(\mathrm{~s}, 1 \mathrm{H}), 4.32(\mathrm{dq}, J=14.4,7.2 \mathrm{~Hz}, 1 \mathrm{H}), 2.88(\mathrm{dq}, J=$ 14.2, 7.1 Hz, 1H), $\left.0.88(\mathrm{t}, J=7.2 \mathrm{~Hz}, 3 \mathrm{H}) ;{ }^{13} \mathrm{C} \mathrm{NMR} \mathrm{(101} \mathrm{MHz,} \mathrm{CDCl}_{3}\right) \delta$ 166.6, $147.4,143.3,134.5,132.5,131.0,130.5,130.3,130.2,129.3,129.0,128.9,128.6$ 128.3, 126.3, 126.3, 125.8, 113.3, 41.6, 13.0; HRMS (ESI) calcd for $\mathrm{C}_{25} \mathrm{H}_{20} \mathrm{~N}_{4} \mathrm{O} m / z$ : 393.1710; Found $393.1707(\mathrm{M}+\mathrm{H})^{+}$. 
(Z)-6-cycloheptyl-10-fluoro-3,5-diphenylbenzo[g][1,2,3] triazolo[1,5-a][1,5]diazo cin-7(6H)-one (3e)<smiles>O=C1c2ccc(F)cc2-n2nnc(-c3ccccc3)c2C=C(c2ccccc2)N1C1CCCCCC1</smiles>

Pale yellow solid (80 mg, $80 \%$ ), (eluent: $8-10 \%$ ethyl acetate in hexanes); mp 190-192 ${ }^{\circ} \mathrm{C} ;{ }^{1} \mathrm{H}$ NMR (400 MHz, $\left.\mathrm{CDCl}_{3}\right) \delta 8.03(\mathrm{~d}, J=7.2 \mathrm{~Hz}, 2 \mathrm{H}), 7.75(\mathrm{dd}, J=8.6,5.7$ $\mathrm{Hz}, 1 \mathrm{H}), 7.56-7.50(\mathrm{~m}, 4 \mathrm{H}), 7.48-7.40(\mathrm{~m}, 4 \mathrm{H}), 7.28$ $(\mathrm{d}, J=2.5 \mathrm{~Hz}, 1 \mathrm{H}), 7.22(\mathrm{dd}, J=8.5,2.5 \mathrm{~Hz}, 1 \mathrm{H}), 6.63$ (s, 1H), $4.04-3.97(\mathrm{~m}, 1 \mathrm{H}), 1.83-1.65(\mathrm{~m}, 4 \mathrm{H}), 1.50-1.27(\mathrm{~m}, 8 \mathrm{H}) ;{ }^{13} \mathrm{C} \mathrm{NMR}$ $\left(101 \mathrm{MHz}, \mathrm{CDCl}_{3}\right) \delta 165.3,161.8,148.7,143.4,136.7,130.6,130.5,130.3,130.1$ 129.0, 128.9, 128.5, 126.9, 126.3, $117.6\left(\mathrm{~d},{ }^{2} J=20.2 \mathrm{~Hz}\right), 114.7,113.3\left(\mathrm{~d},{ }^{2} J=20.2\right.$ $\mathrm{Hz}$ ), 61.5, 33.2, 32.9, 27.3, 27.1, 25.1, 24.9; HRMS (ESI) calcd for $\mathrm{C}_{30} \mathrm{H}_{27} \mathrm{FN}_{4} \mathrm{O} \mathrm{m} / z$ : 479.2242 ; Found $479.2246(\mathrm{M}+\mathrm{H})^{+}$.

(Z)-10-fluoro-5-phenyl-6-(3-phenylpropyl)-3-(p-tolyl)benzo[g] $[1,2,3]$ triazolo[1,5 -a][1,5]diazocin-7(6H)-one (3f)

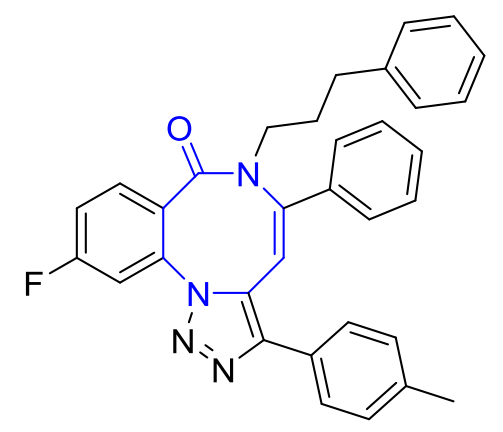

Yellow solid (80 mg, $80 \%$ ), (eluent: $8-10 \%$ ethyl acetate in hexanes); mp $188-190{ }^{\circ} \mathrm{C} ;{ }^{1} \mathrm{H}$ NMR (400 $\left.\mathrm{MHz}, \mathrm{CDCl}_{3}\right) \delta 7.87(\mathrm{~d}, J=8.1 \mathrm{~Hz}, 2 \mathrm{H}), 7.74(\mathrm{dd}, J=$ 8.5, $5.7 \mathrm{~Hz}, 1 \mathrm{H}), 7.47-7.43(\mathrm{~m}, 5 \mathrm{H}), 7.30-7.27(\mathrm{~m}$, 3H), $7.23(\mathrm{~s}, 1 \mathrm{H}), 7.06-7.05(\mathrm{~m}, 3 \mathrm{H}), 6.77(\mathrm{~s}, 3 \mathrm{H})$, $4.34-4.27(\mathrm{~m}, 1 \mathrm{H}), 2.84-2.78(\mathrm{~m}, 1 \mathrm{H}), 2.43(\mathrm{~s}, 3 \mathrm{H}), 2.27(\mathrm{t}, J=7.6 \mathrm{~Hz}, 2 \mathrm{H})$, $1.63-1.52(\mathrm{~m}, 2 \mathrm{H}) ;{ }^{13} \mathrm{C}$ NMR $\left(101 \mathrm{MHz}, \mathrm{CDCl}_{3}\right) \delta 166.2,163.3\left(\mathrm{~d},{ }^{1} \mathrm{~J}=252.5 \mathrm{~Hz}\right)$, $147.6,143.5,140.5,138.4,134.3,130.9,130.8,130.3,129.7,129.4,128.1,128.0$, 126.3, 126.2, 125.6, $117.6\left(\mathrm{~d},{ }^{2} J=20.2 \mathrm{~Hz}\right), 113.5\left(\mathrm{~d},{ }^{2} J=20.2 \mathrm{~Hz}\right), 113.3,46.3,32.5$, 29.0, 21.3; HRMS (ESI) calcd for $\mathrm{C}_{33} \mathrm{H}_{27} \mathrm{FN}_{4} \mathrm{O} \mathrm{m} / \mathrm{z}$ : 515.2242 ; Found 515.2243 $(\mathrm{M}+\mathrm{H})^{+}$. 
(Z)-3-(4-methoxyphenyl)-5-phenyl-6-(3-phenylpropyl)benzo[g][1,2,3]triazolo[1,

\section{5-a][1,5]diazocin-7(6H)-one (3g)}

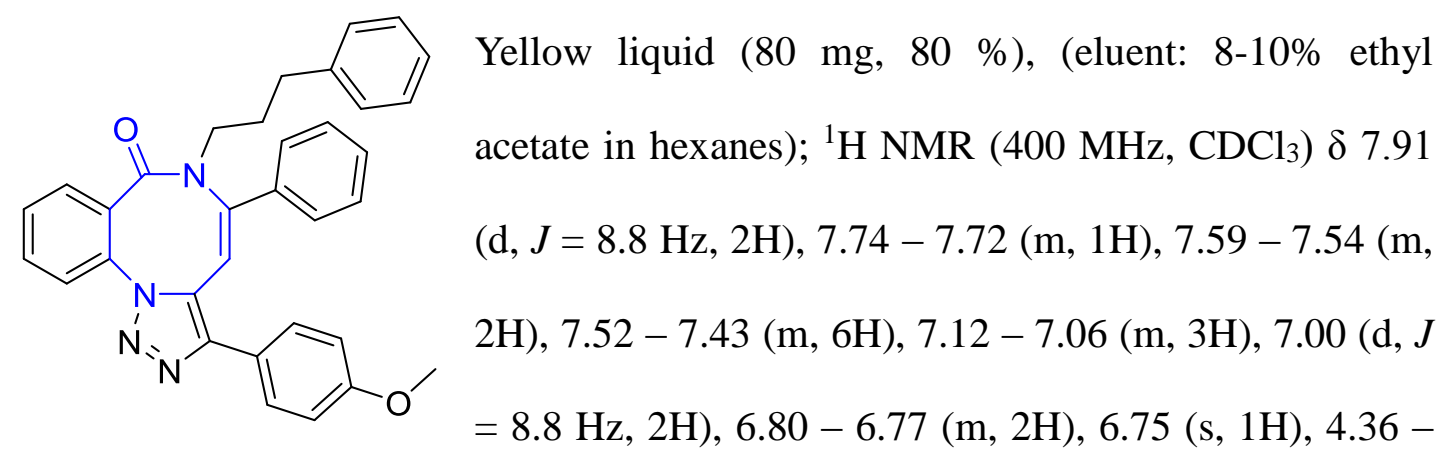

$4.29(\mathrm{~m}, 1 \mathrm{H}), 3.88(\mathrm{~s}, 3 \mathrm{H}), 2.85-2.78(\mathrm{~m}, 1 \mathrm{H}), 2.28(\mathrm{t}, J=6.9 \mathrm{~Hz}, 2 \mathrm{H}), 1.66-1.59$ $(\mathrm{m}, 2 \mathrm{H}) ;{ }^{13} \mathrm{C} \mathrm{NMR}\left(101 \mathrm{MHz}, \mathrm{CDCl}_{3}\right) \delta 167.0,159.6,147.5,143.2,140.6,134.5$, $132.6,132.5,131.0,130.2,130.1,129.3,128.7,128.1,128.0,128.0,127.6,126.3$, 125.9, 125.6, 123.0, 114.4, 113.6, 55.3, 46.1, 32.5, 29.1; HRMS (ESI) calcd for $\mathrm{C}_{33} \mathrm{H}_{28} \mathrm{~N}_{4} \mathrm{O}_{2} m / z: 513.2285$; Found $513.2290(\mathrm{M}+\mathrm{H})^{+}$.

(Z)-10-chloro-3-cyclohexyl-6-(3-methoxypropyl)-5-(4-(trifluoromethyl)phenyl)b enzo[g][1,2,3]triazolo[1,5-a][1,5]diazocin-7(6H)-one (3h)

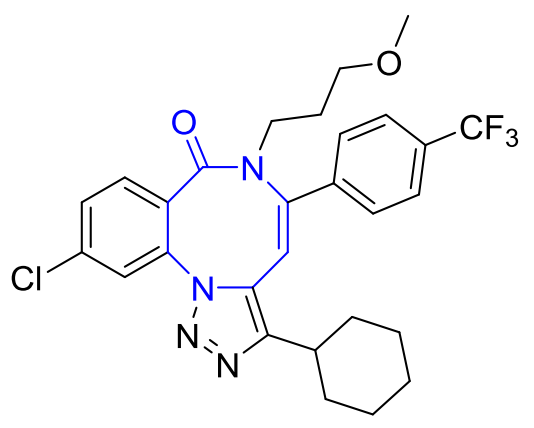

Yellow solid (84 mg, $84 \%$ ), (eluent: $8-10 \%$ ethyl acetate in hexanes); mp 180-182 ${ }^{\circ} \mathrm{C} ;{ }^{1} \mathrm{H}$ NMR (400 $\left.\mathrm{MHz}, \mathrm{CDCl}_{3}\right) \delta 8.10(\mathrm{~d}, J=2.1 \mathrm{~Hz}, 1 \mathrm{H}), 8.00(\mathrm{~d}, J$ $=8.5 \mathrm{~Hz}, 1 \mathrm{H}), 7.53-7.48(\mathrm{~m}, 3 \mathrm{H}), 7.15(\mathrm{~s}, 1 \mathrm{H})$, $7.02(\mathrm{~d}, J=8.2 \mathrm{~Hz}, 2 \mathrm{H}), 4.33-4.26(\mathrm{~m}, 1 \mathrm{H}), 3.84-$ $3.78(\mathrm{~m}, 1 \mathrm{H}), 3.42(\mathrm{t}, J=5.9 \mathrm{~Hz}, 2 \mathrm{H}), 3.31(\mathrm{~s}, 3 \mathrm{H}), 2.04-1.88(\mathrm{~m}, 4 \mathrm{H}), 1.77-1.68$ $(\mathrm{m}, 2 \mathrm{H}), 1.65(\mathrm{~s}, 1 \mathrm{H}), 1.58-1.34(\mathrm{~m}, 6 \mathrm{H}) ;{ }^{13} \mathrm{C} \mathrm{NMR}\left(101 \mathrm{MHz}, \mathrm{CDCl}_{3}\right) \delta 164.2$, $148.0,138.8,136.8,133.7,133.7,133.5,131.0,129.1,128.9,128.1,125.3$ (q, ${ }^{3} J=$ $3.7 \mathrm{~Hz}), 125.3,122.0,69.9,58.6,48.8,35.2,33.0,30.3,28.5,26.4,26.2,25.5$; HRMS (ESI) calcd for $\mathrm{C}_{28} \mathrm{H}_{28} \mathrm{ClF}_{3} \mathrm{~N}_{4} \mathrm{O}_{2}$ m/z: 545.1926; Found 545.1958 (M+H) . 


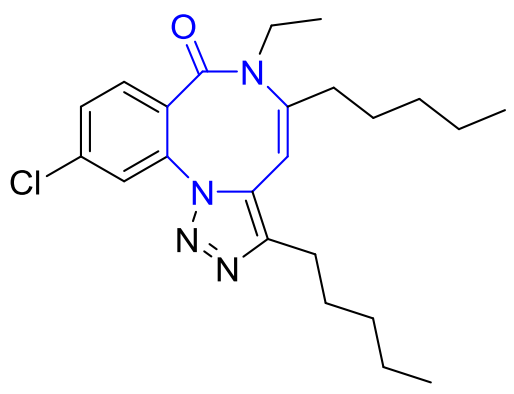

Pale Yellow liquid (81 mg, $81 \%$ ), (eluent: $8-10 \%$ ethyl acetate in hexanes); ${ }^{1} \mathrm{H}$ NMR (400 MHz, $\left.\mathrm{CDCl}_{3}\right) \delta 7.55-7.46(\mathrm{~m}, 2 \mathrm{H}), 7.42-7.40(\mathrm{~m}, 1 \mathrm{H})$, $6.08(\mathrm{~s}, 1 \mathrm{H}), 4.16(\mathrm{dq}, J=14.3,7.2 \mathrm{~Hz}, 1 \mathrm{H}), 3.08$ $(\mathrm{dq}, J=14.1,7.0 \mathrm{~Hz}, 1 \mathrm{H}), 2.72-2.60(\mathrm{~m}, 2 \mathrm{H})$, $2.45-2.38(\mathrm{~m}, 1 \mathrm{H}), 2.25-2.17(\mathrm{~m}, 1 \mathrm{H}), 1.81-1.68(\mathrm{~m}, 4 \mathrm{H}), 1.45-1.35(\mathrm{~m}, 5 \mathrm{H})$, $1.09-0.95(\mathrm{~m}, 5 \mathrm{H}), 0.90(\mathrm{t}, J=6.7 \mathrm{~Hz}, 4 \mathrm{H}), 0.77(\mathrm{t}, J=7.3 \mathrm{~Hz}, 3 \mathrm{H}) ;{ }^{13} \mathrm{C} \mathrm{NMR}$ $\left(101 \mathrm{MHz}, \mathrm{CDCl}_{3}\right) \delta 166.0,148.5,144.7,132.9,132.7,130.5,129.5,129.4,128.8$, $125.7,112.8,39.6,34.0,31.6,30.9,28.6,26.5,25.4,22.4,22.3,13.9,13.8,12.6$; HRMS (ESI) calcd for $\mathrm{C}_{23} \mathrm{H}_{31} \mathrm{ClN}_{4} \mathrm{O} \mathrm{m} / z$ : 415.2259; Found 415.2310 (M+H) ${ }^{+}$.

(Z)-10-chloro-3-pentyl-5-phenyl-6-(3-phenylpropyl)benzo[g][1,2,3]triazolo[1,5-a ]$[1,5]$ diazocin-7(6H)-one $(3 \mathbf{j})$

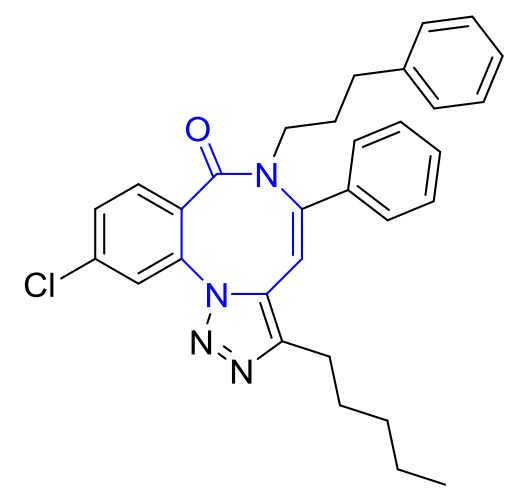

Yellow solid (83 mg, $83 \%$ ), (eluent: $8-10 \%$ ethyl acetate in hexanes); mp 196-199 ${ }^{\circ} \mathrm{C} ;{ }^{1} \mathrm{H}$ NMR (400 $\left.\mathrm{MHz}, \mathrm{CDCl}_{3}\right) \delta 7.64(\mathrm{~d}, J=8.3 \mathrm{~Hz}, 1 \mathrm{H}), 7.52-$ $7.42(\mathrm{~m}, 2 \mathrm{H}), 7.42(\mathrm{~s}, 4 \mathrm{H}), 7.22-7.11(\mathrm{~m}, 3 \mathrm{H})$, $6.99(\mathrm{~d}, J=7.2 \mathrm{~Hz}, 2 \mathrm{H}), 6.57(\mathrm{~s}, 1 \mathrm{H}), 4.30-4.22$ (m, 1H), $2.82-2.74(\mathrm{~m}, 1 \mathrm{H}), 2.72-2.60(\mathrm{~m}, 2 \mathrm{H})$ $2.37-2.33(\mathrm{~m}, 2 \mathrm{H}), 1.79-1.70(\mathrm{~m}, 2 \mathrm{H}), 1.65-1.57(\mathrm{~m}, 3 \mathrm{H}), 1.35-1.30(\mathrm{~m}, 3 \mathrm{H})$, $0.91(\mathrm{t}, J=7.0 \mathrm{~Hz}, 3 \mathrm{H}) ;{ }^{13} \mathrm{C} \mathrm{NMR}\left(101 \mathrm{MHz}, \mathrm{CDCl}_{3}\right) \delta 166.2,147.5,145.1,140.6$, $136.7,134.4,133.8,130.8,130.3,130.2,130.0,129.6,129.3,128.3,128.0,126.2$, 126.1, 125.9, 112.2, 46.3, 32.7, 31.6, 29.0, 28.3, 25.5, 22.3, 14.0; HRMS (ESI) calcd for $\mathrm{C}_{31} \mathrm{H}_{31} \mathrm{ClN}_{4} \mathrm{O} m / z$ : 511.2259; Found $511.2258(\mathrm{M}+\mathrm{H})^{+}$. 
(Z)-6-(benzo[d][1,3]dioxol-5-ylmethyl)-5-phenyl-3-(4-(trifluoromethyl)phenyl)n aphtho[2,3-g][1,2,3]triazolo[1,5-a][1,5]diazocin-7(6H)-one (3k)

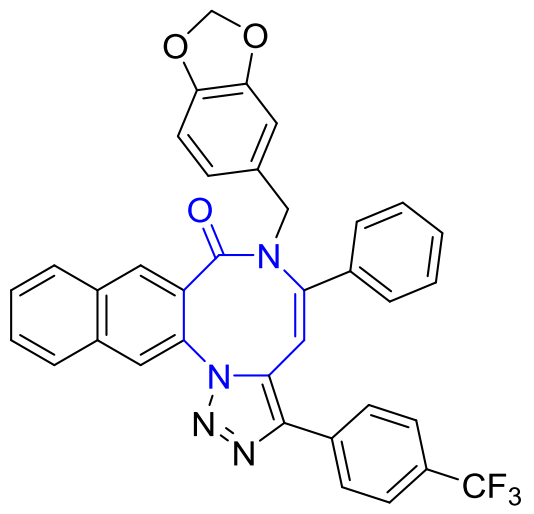

Pale yellow solid (84 mg, $84 \%$ ), (eluent: $8-10 \%$ ethyl acetate in hexanes); mp 200-203 ${ }^{\circ} \mathrm{C} ;{ }^{1} \mathrm{H}$ NMR $\left(400 \mathrm{MHz}, \mathrm{CDCl}_{3}\right) \delta 8.31(\mathrm{~s}, 1 \mathrm{H}), 8.02-7.96(\mathrm{~m}$, 2H), $7.90(\mathrm{~d}, J=8.8 \mathrm{~Hz}, 1 \mathrm{H}), 7.65-7.59(\mathrm{~m}, 6 \mathrm{H})$, 7.50 - 7.4. (m, 5H), $6.52(\mathrm{~s}, 1 \mathrm{H}), 6.35(\mathrm{~d}, J=7.8 \mathrm{~Hz}$, $1 \mathrm{H}), 6.30-6.19(\mathrm{~m}, 2 \mathrm{H}), 5.79(\mathrm{~d}, J=14.3 \mathrm{~Hz}, 1 \mathrm{H})$, $5.61(\mathrm{~s}, 1 \mathrm{H}), 5.27(\mathrm{~s}, 1 \mathrm{H}), 3.68(\mathrm{~d}, J=14.3 \mathrm{~Hz}, 1 \mathrm{H}) ;{ }^{13} \mathrm{C} \mathrm{NMR}\left(101 \mathrm{MHz}, \mathrm{CDCl}_{3}\right) \delta$ $167.0,147.7,147.4,147.3,142.1,134.3,133.6,133.5,133.1,130.5,129.9,129.9$, $129.7,129.5,129.1,128.7,128.4,128.4,128.2,128.2,126.4,126.0,125.3\left(\mathrm{q},{ }^{3} J=\right.$ $4.3 \mathrm{~Hz}), 122.0,113.6,108.5,107.9,100.8,49.0 ;$ HRMS (ESI) calcd for $\mathrm{C}_{36} \mathrm{H}_{23} \mathrm{~F}_{3} \mathrm{~N}_{4} \mathrm{O}_{3} \mathrm{~m} / z$ : 617.1795; Found 617.1769 (M+H) ${ }^{+}$. 

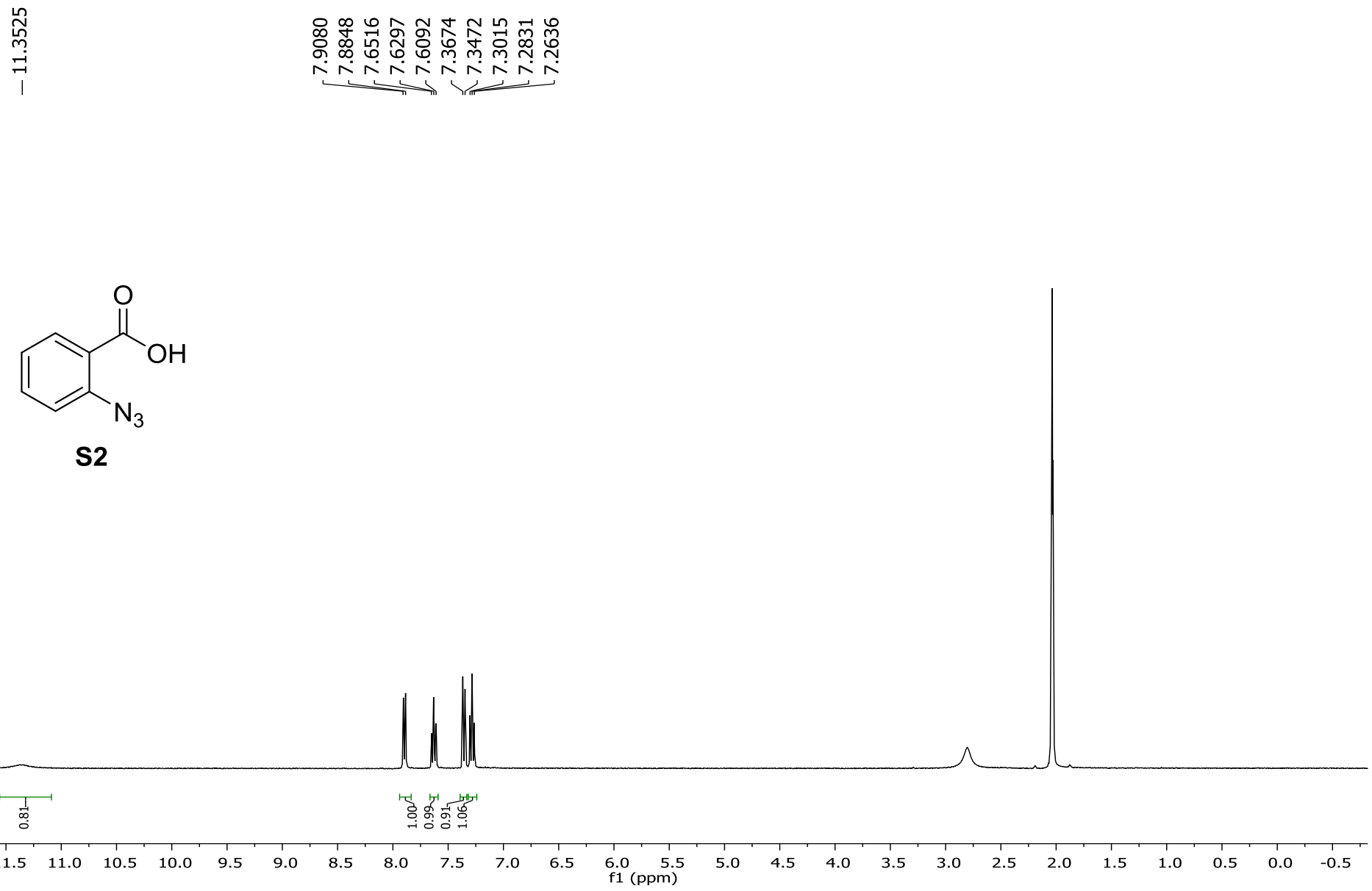

${ }^{1} \mathrm{H}$ NMR spectrum $\left(400 \mathrm{MHz}\right.$ ) of compound $\mathbf{S 2}$ in Acetone- $d_{6}$ 


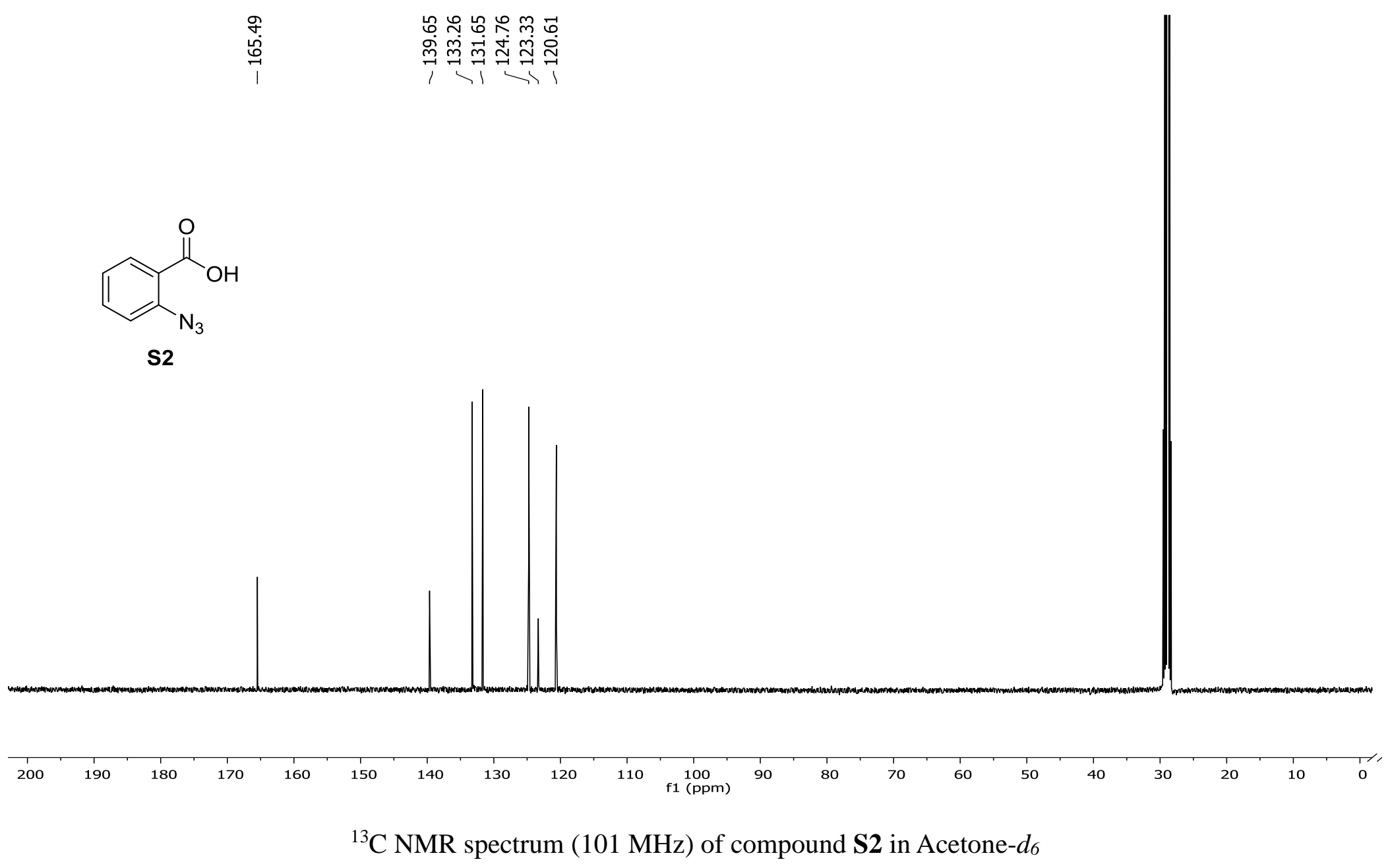




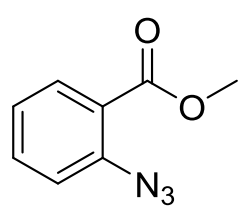

S3

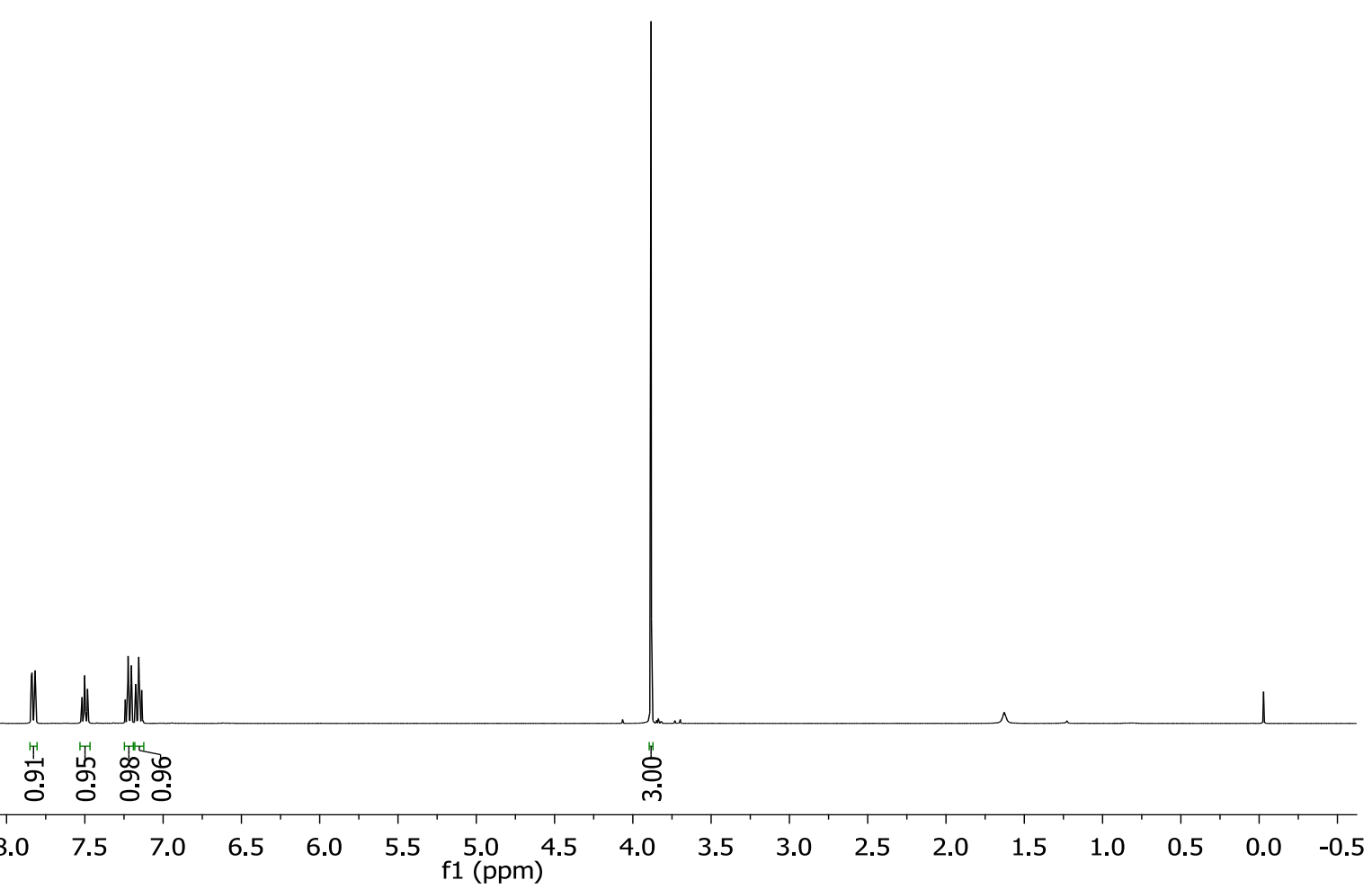

${ }^{1} \mathrm{H}$ NMR spectrum (400 MHz) of compound $\mathbf{S 3}$ in $\mathrm{CDCl}_{3}$ 


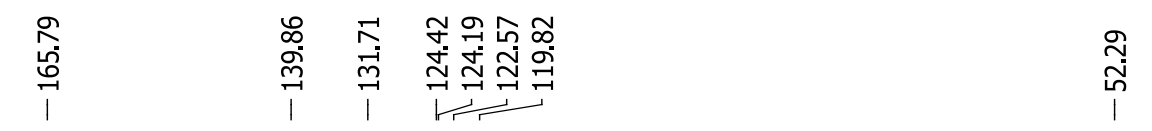
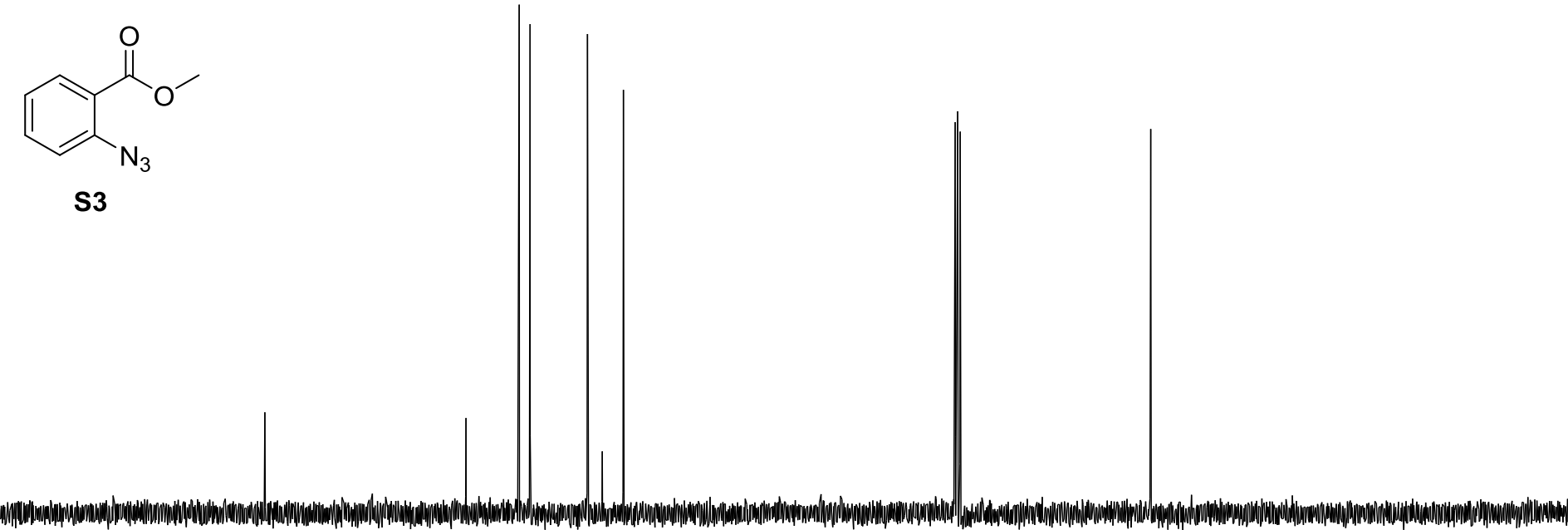

$200 \quad 190 \quad 180 \quad 170 \quad 160$

$150 \quad 140$

$130 \quad 120$

$110 \quad 100$

90

$80 \quad 70$

$60 \quad 50$

$\begin{array}{lllll}40 & 30 & 20 & 10 & 0\end{array}$

${ }^{13} \mathrm{C}$ NMR spectrum (101 MHz) of compound $\mathbf{S 3}$ in $\mathrm{CDCl}_{3}$ 

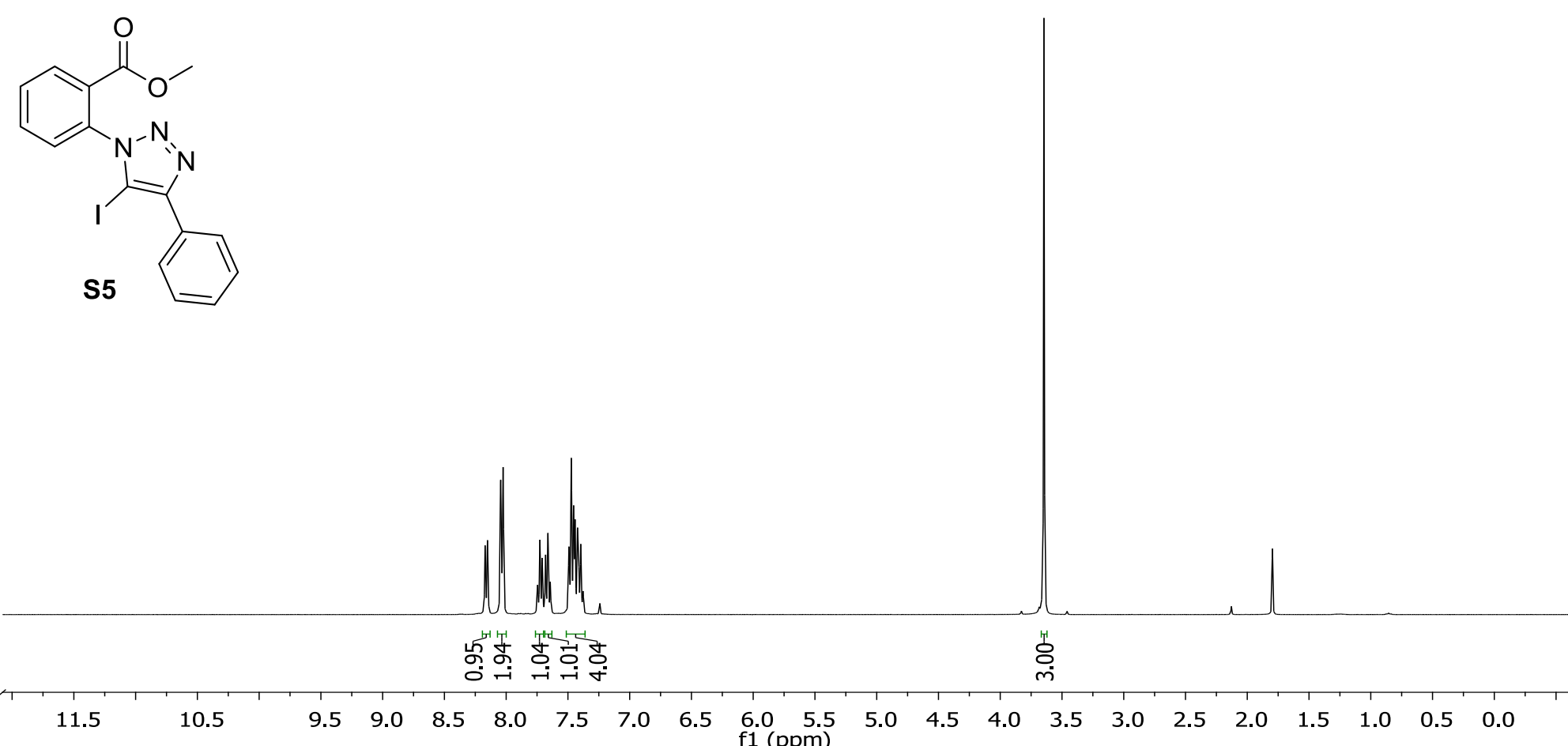

${ }^{1} \mathrm{H}$ NMR spectrum $\left(400 \mathrm{MHz}\right.$ ) of compound $\mathbf{S 5}$ in $\mathrm{CDCl}_{3}$ 


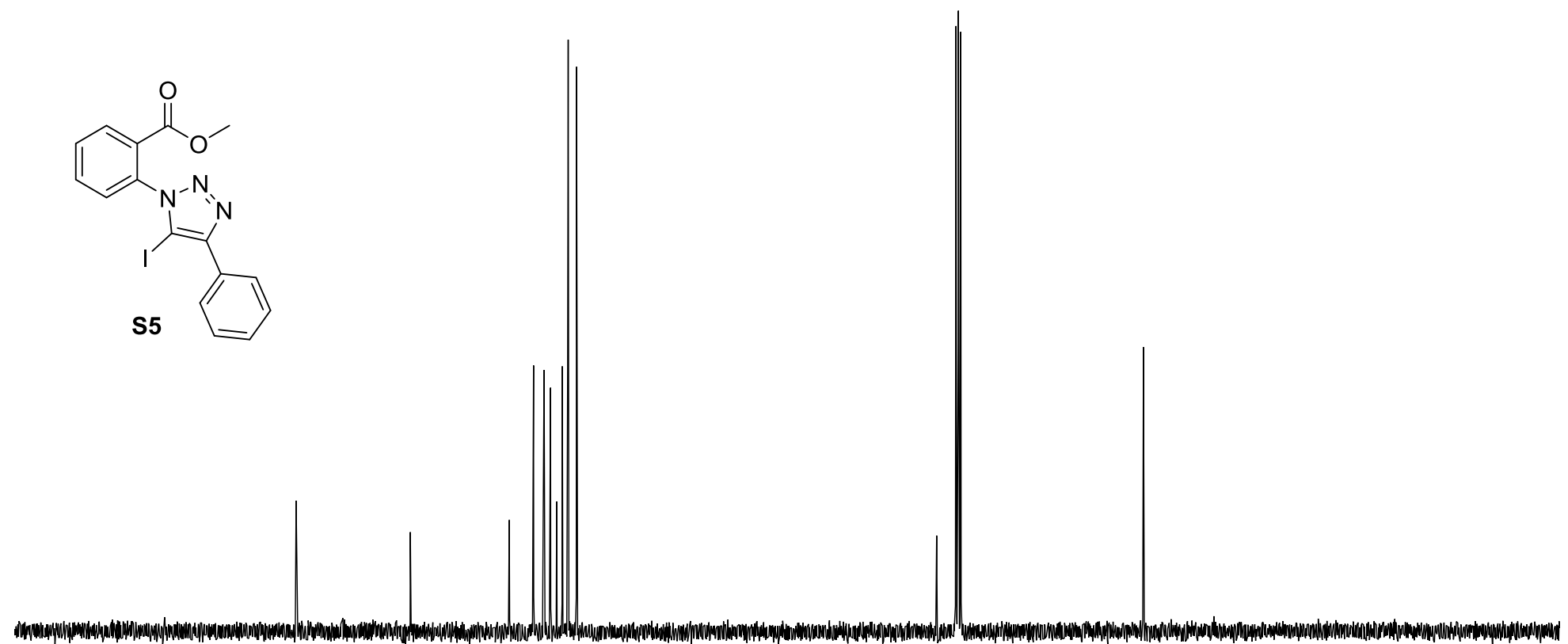

200 $190 \quad 180$ 160 $150 \quad 140$ 130 $110 \quad 100$ 80 60

${ }^{13} \mathrm{C}$ NMR spectrum $\left(101 \mathrm{MHz}\right.$ ) of compound $\mathbf{S 5}$ in $\mathrm{CDCl}_{3}$ 


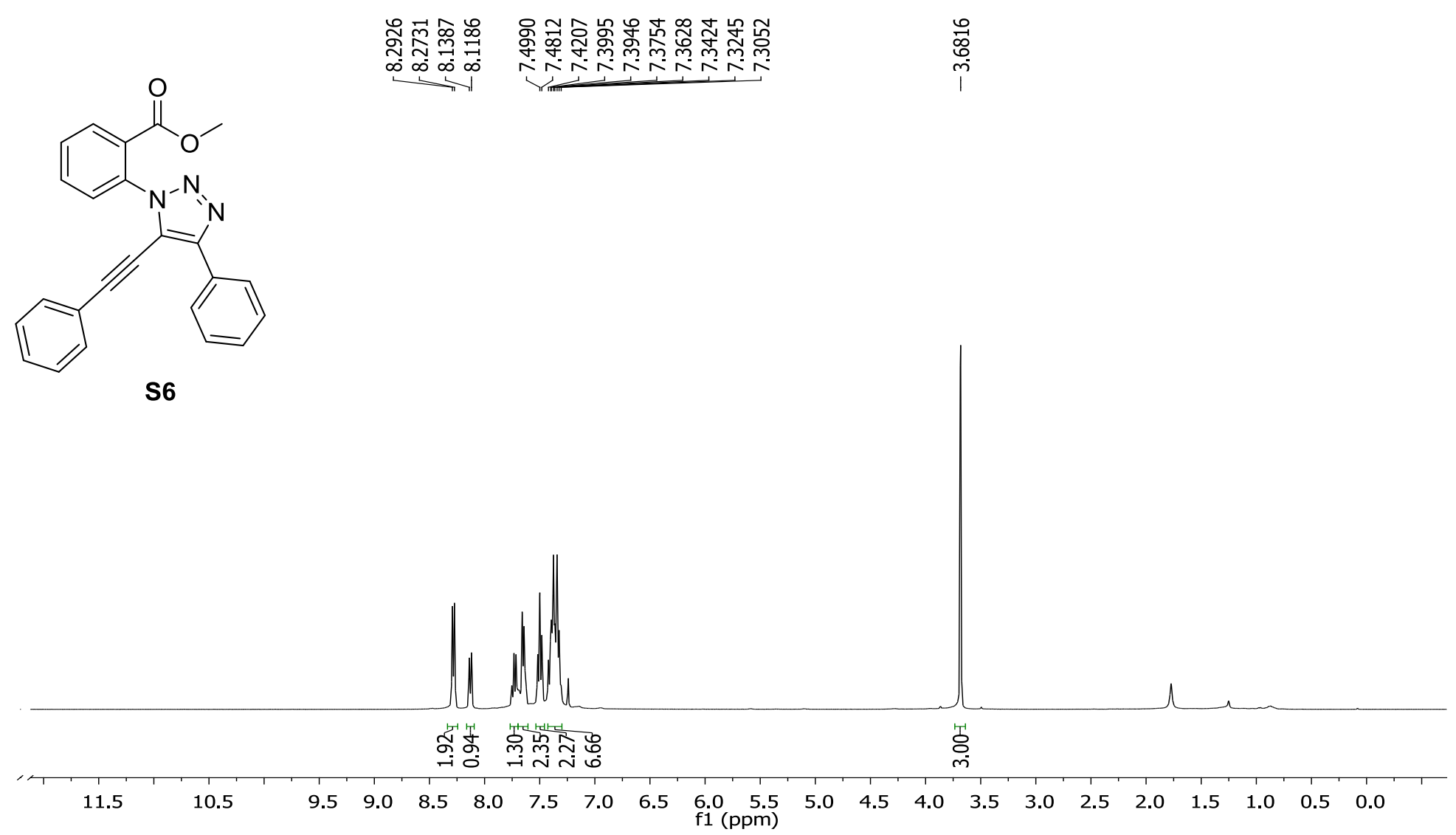

${ }^{1} \mathrm{H}$ NMR spectrum (400 MHz) of compound $\mathbf{S 6}$ in $\mathrm{CDCl}_{3}$ 


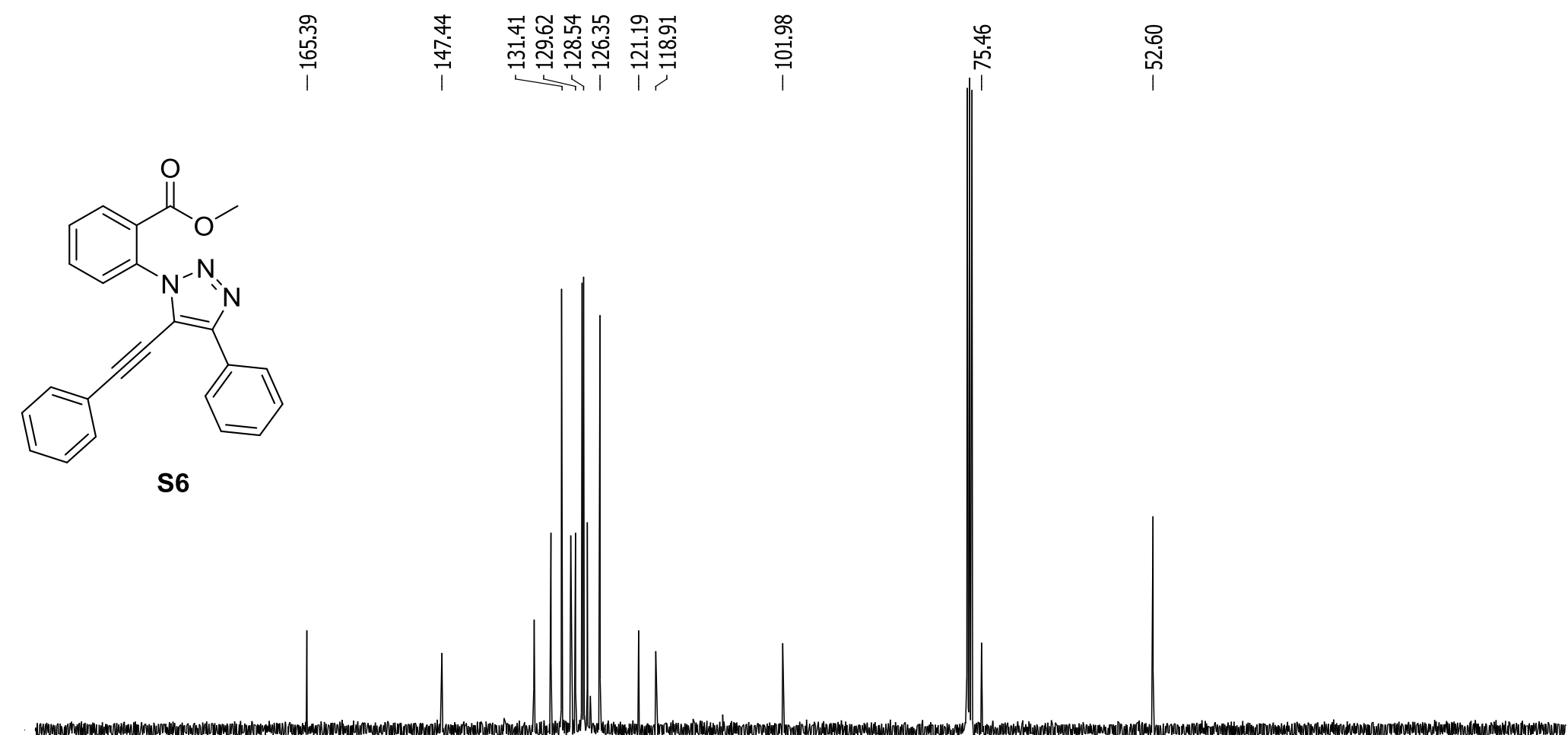

200

$\begin{array}{llll}180 & 170 & 160 & 150\end{array}$

$\begin{array}{llll}140 & 130 & 120 & 110 \\ \mathrm{f} 1 & \begin{array}{c}100 \\ (\mathrm{ppm})\end{array}\end{array}$

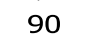

80

70

60

50

40

30

20

10

${ }^{13} \mathrm{C}$ NMR spectrum (101 MHz) of compound $\mathbf{S 6}$ in $\mathrm{CDCl}_{3}$ 

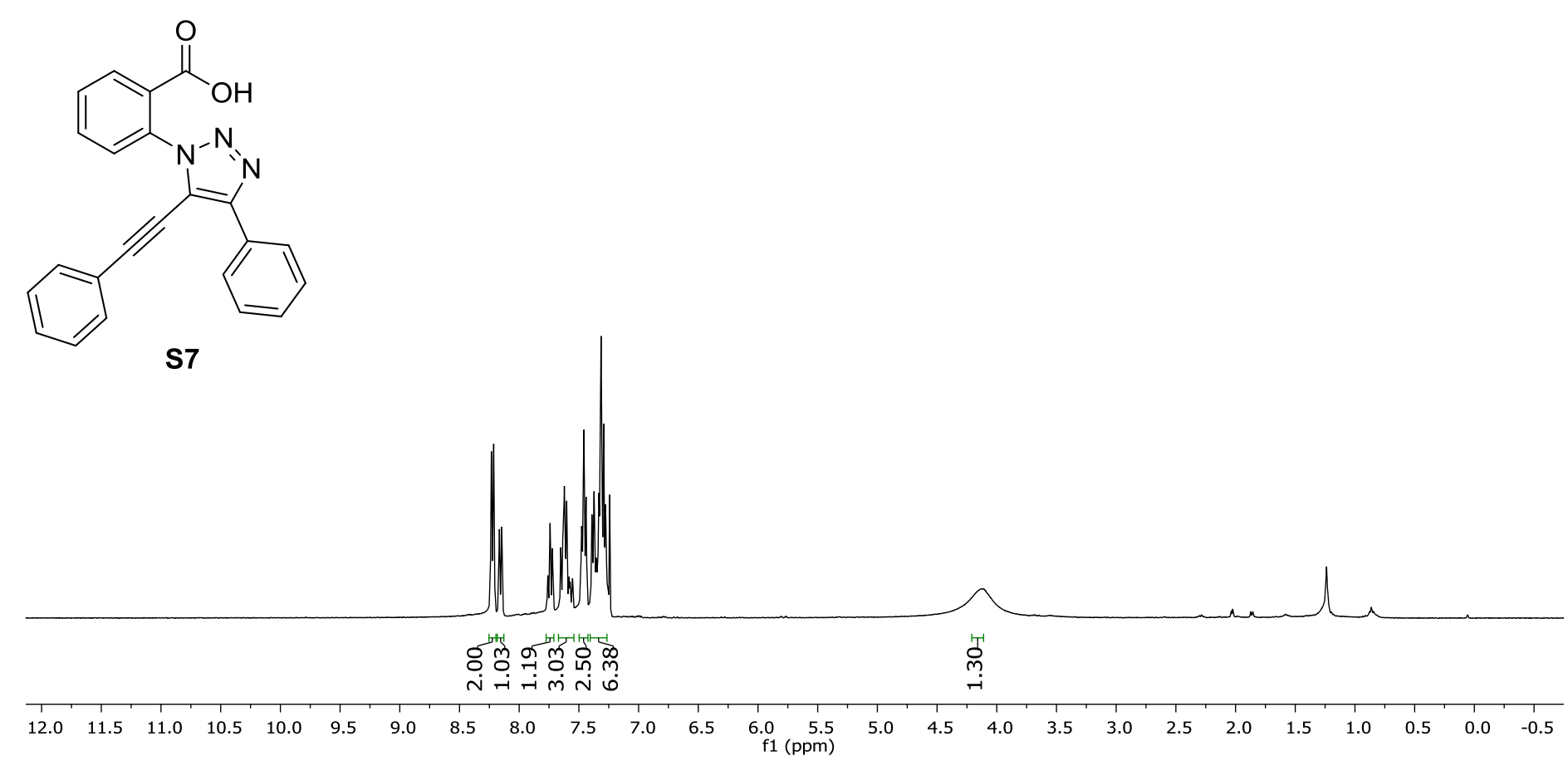

${ }^{1} \mathrm{H}$ NMR spectrum (400 MHz) of compound $\mathbf{S 7}$ in $\mathrm{CDCl}_{3}$ 


$$
11
$$




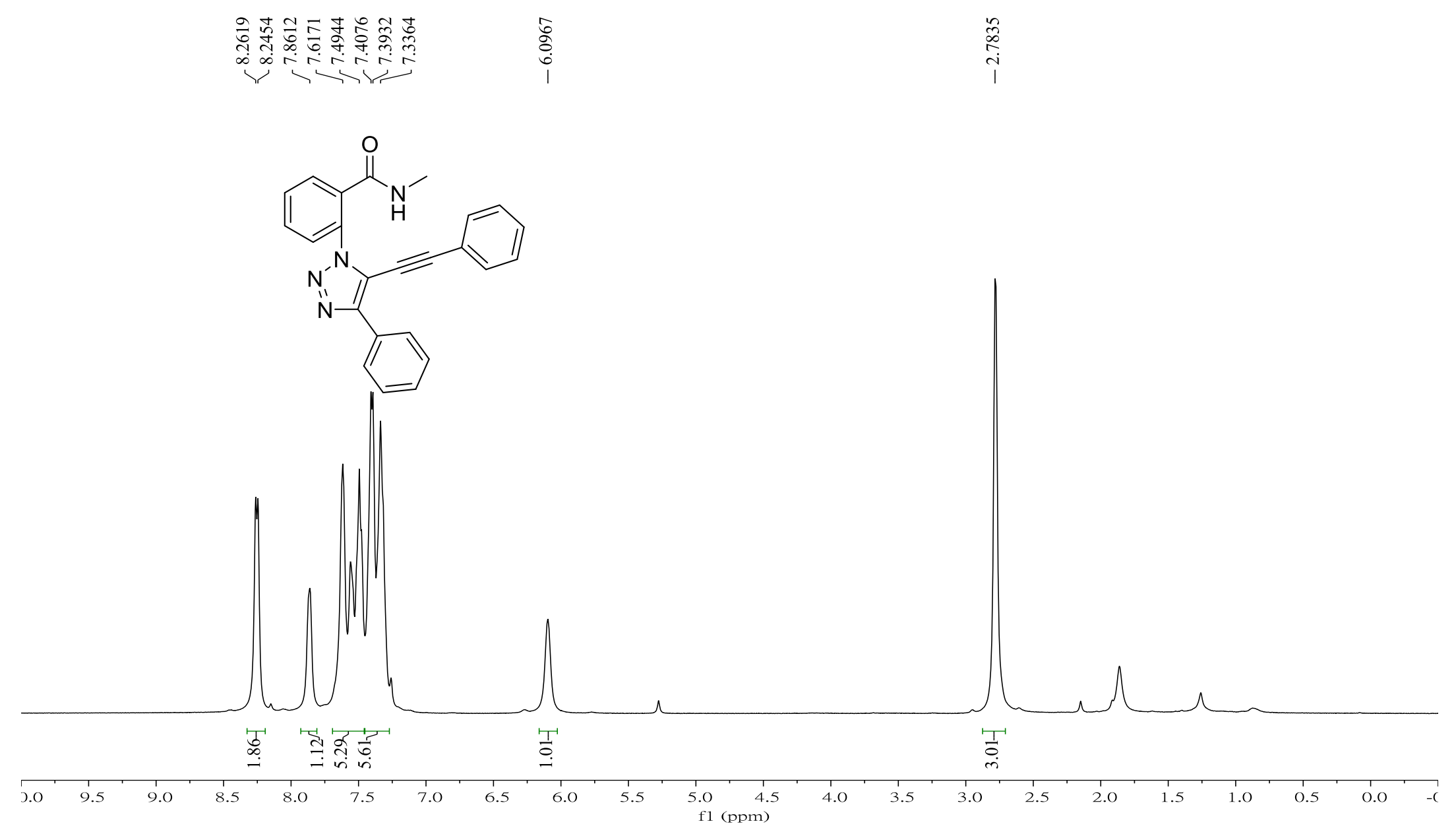

${ }^{1} \mathrm{H}$ NMR Spectrum (400 MHz) of compound $\mathbf{1 a}$ in $\mathrm{CDCl}_{3}$ 


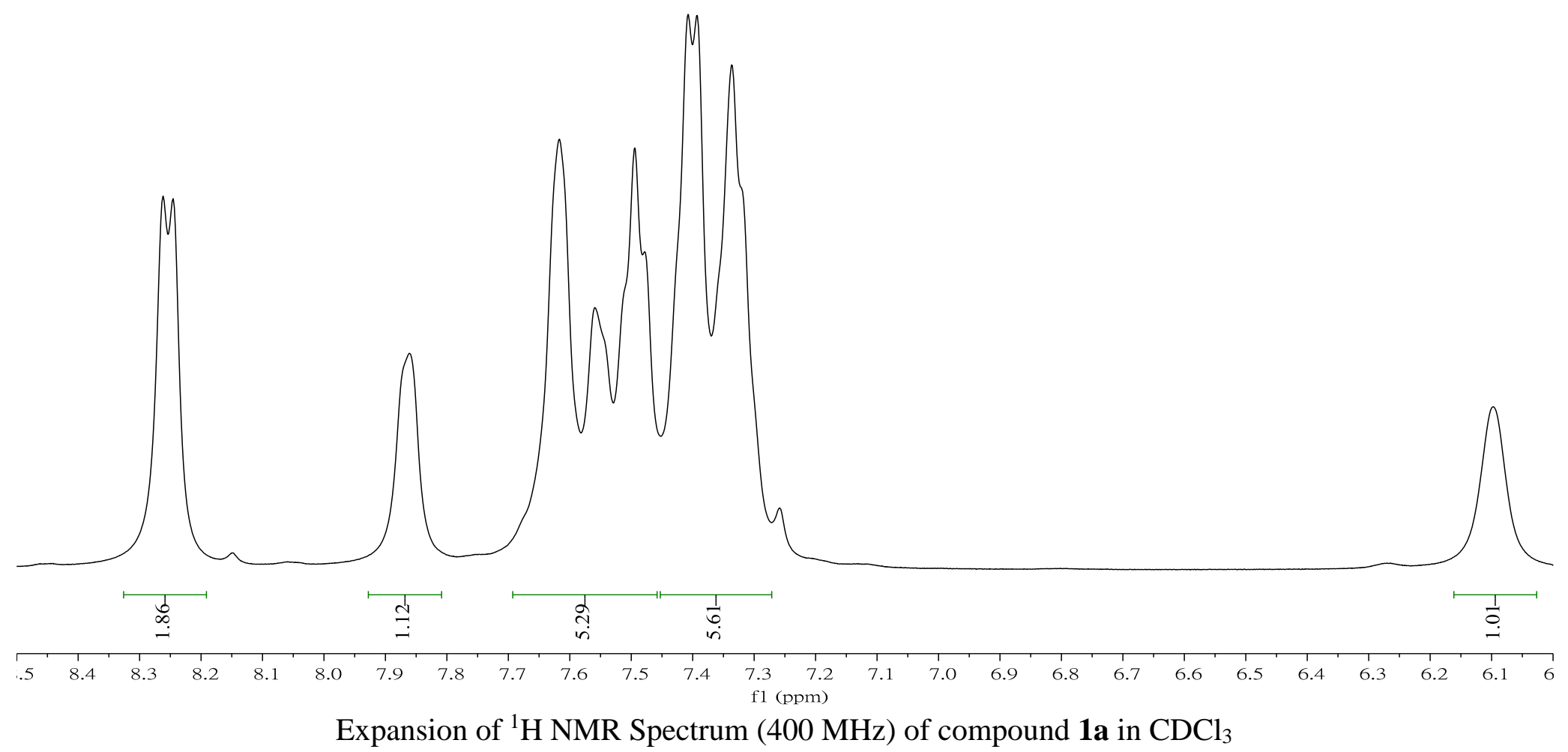




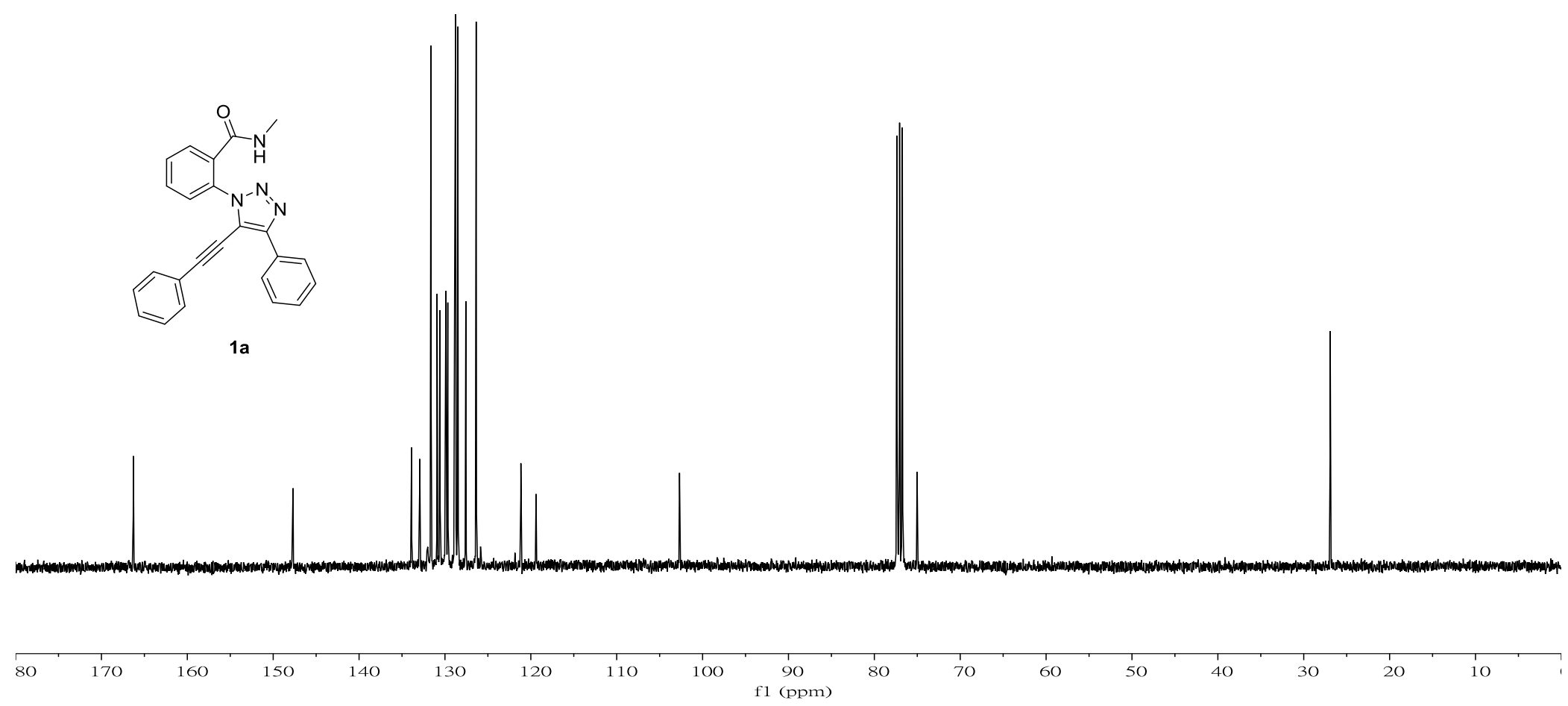

${ }^{13} \mathrm{C}$ NMR Spectrum $(101 \mathrm{MHz})$ of compound $\mathbf{1 a}$ in $\mathrm{CDCl}_{3}$ 


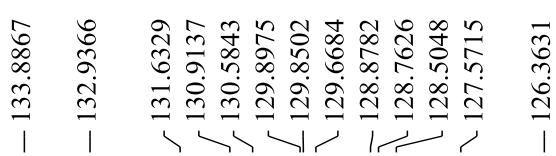

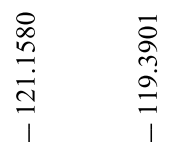

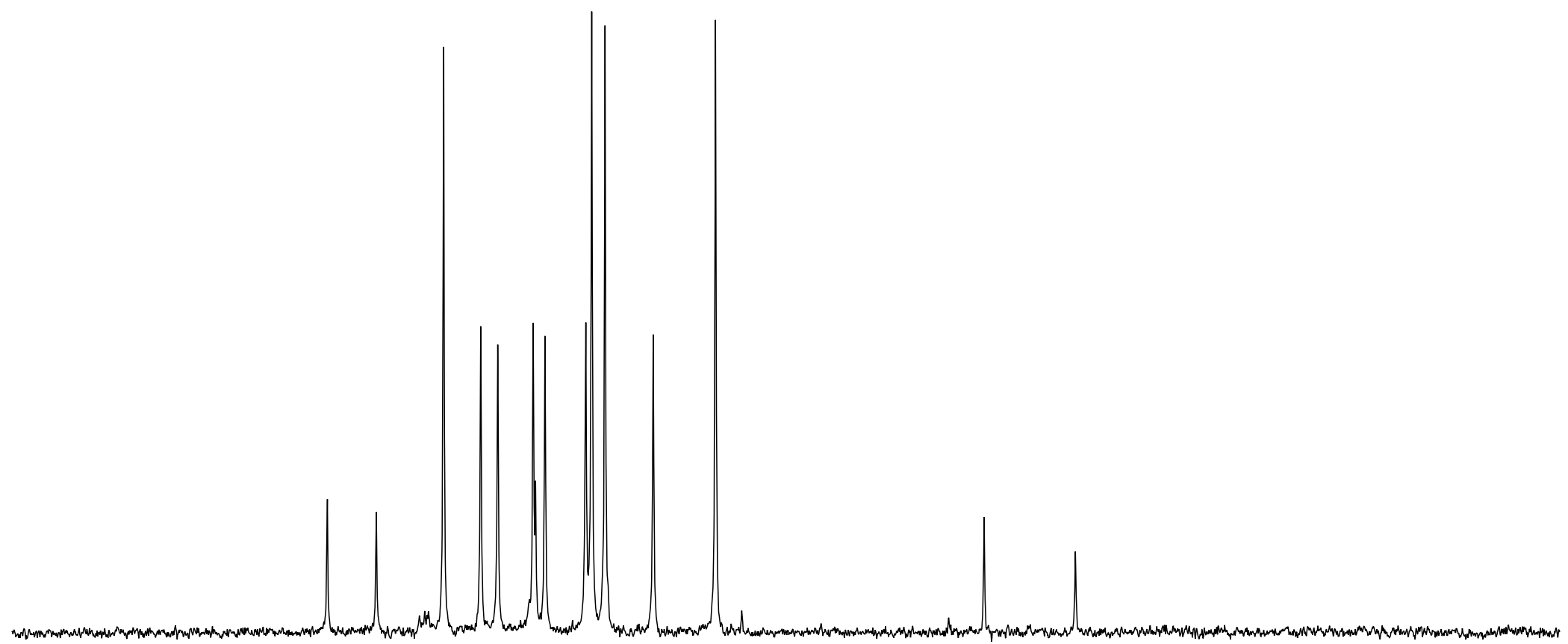

$\begin{array}{llllllllllllllllllllllllllllllllllllllll}40 & 139 & 138 & 137 & 136 & 135 & 134 & 133 & 132 & 131 & 130 & 129 & 128 & 127 & 126 & 125 & 124 & 123 & 122 & 121 & 120 & 119 & 118 & 117 & 116 & 115 & 114 & 113 & 112 & 111\end{array}$ f1 (ppm)

Expansion of ${ }^{13} \mathrm{C}$ NMR Spectrum (101 MHz) of compound 1a in $\mathrm{CDCl}_{3}$ 


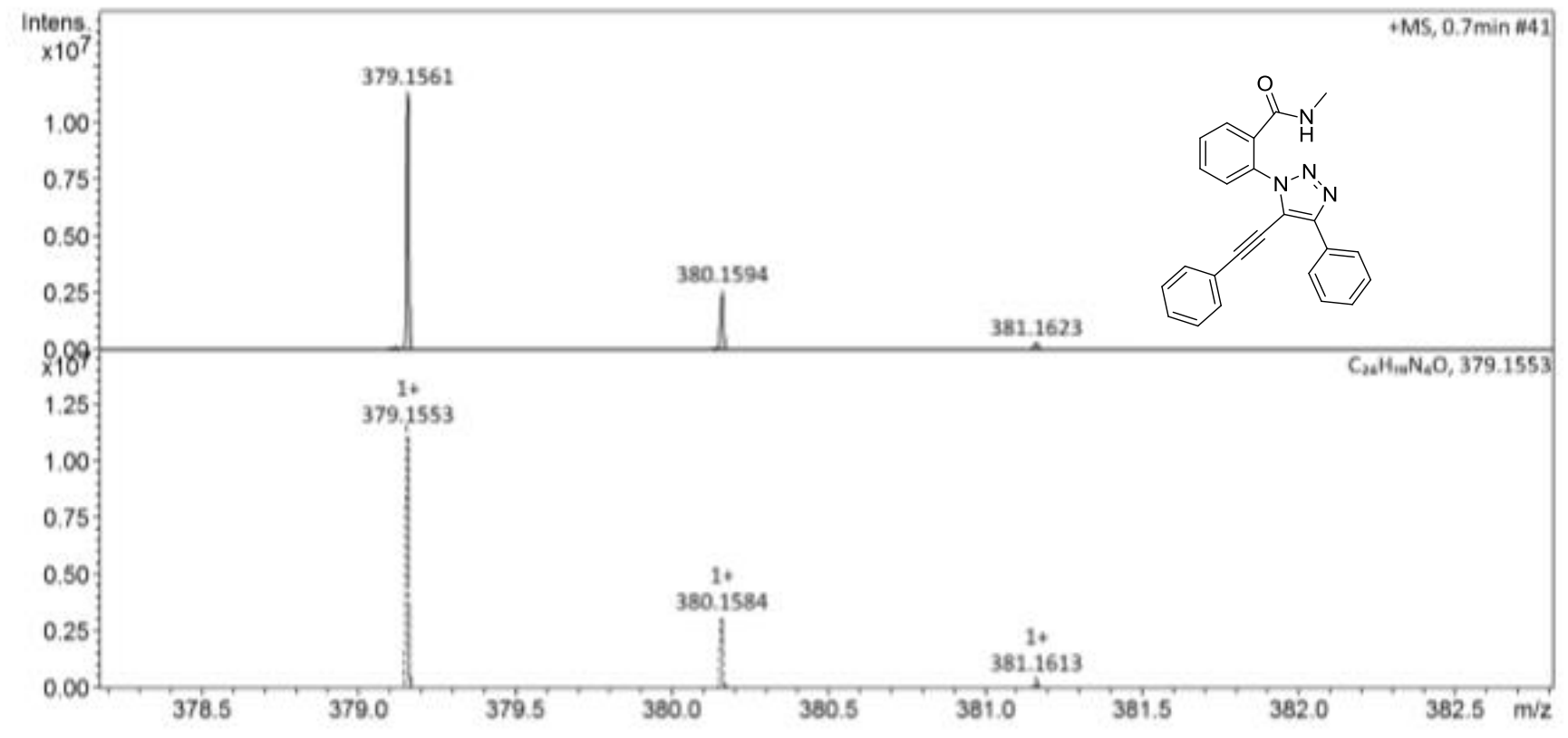

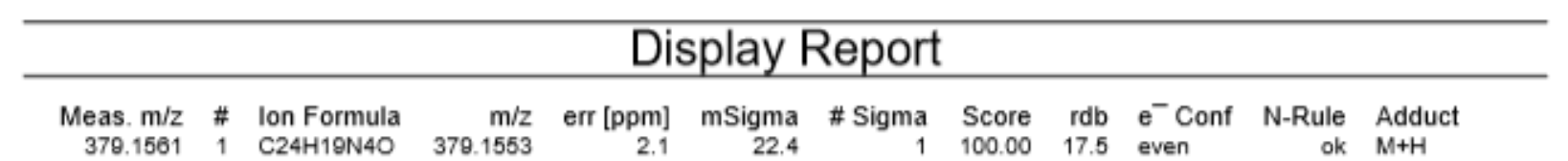

HRMS of compound 1a 


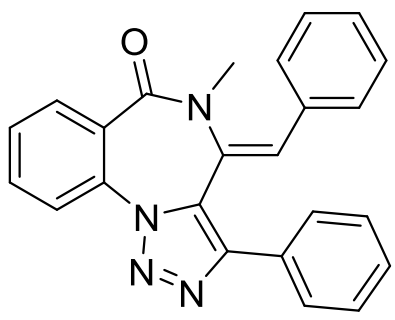

$2 a$

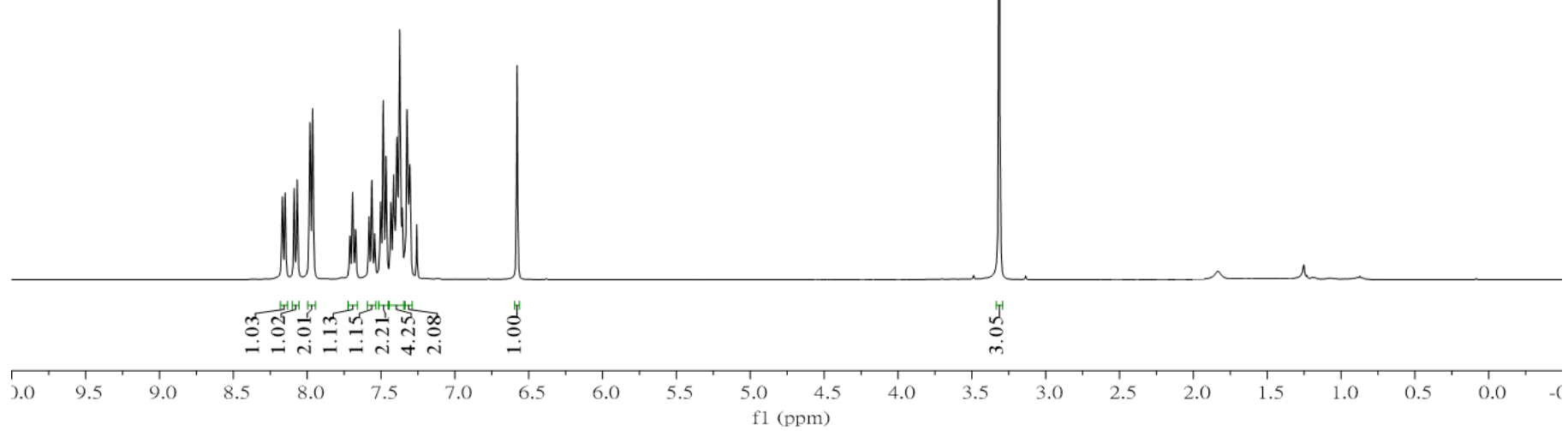

${ }^{1} \mathrm{H}$ NMR Spectrum (400 MHz) of compound 2a in $\mathrm{CDCl}_{3}$ 


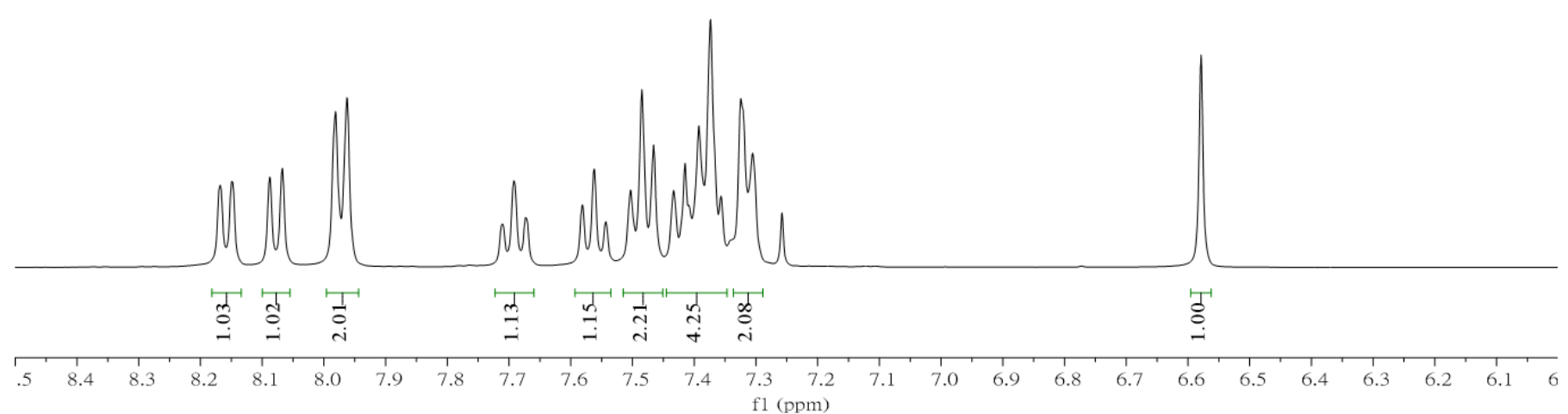

Expansion of ${ }^{1} \mathrm{H}$ NMR Spectrum $(400 \mathrm{MHz})$ of compound 2a in $\mathrm{CDCl}_{3}$ 


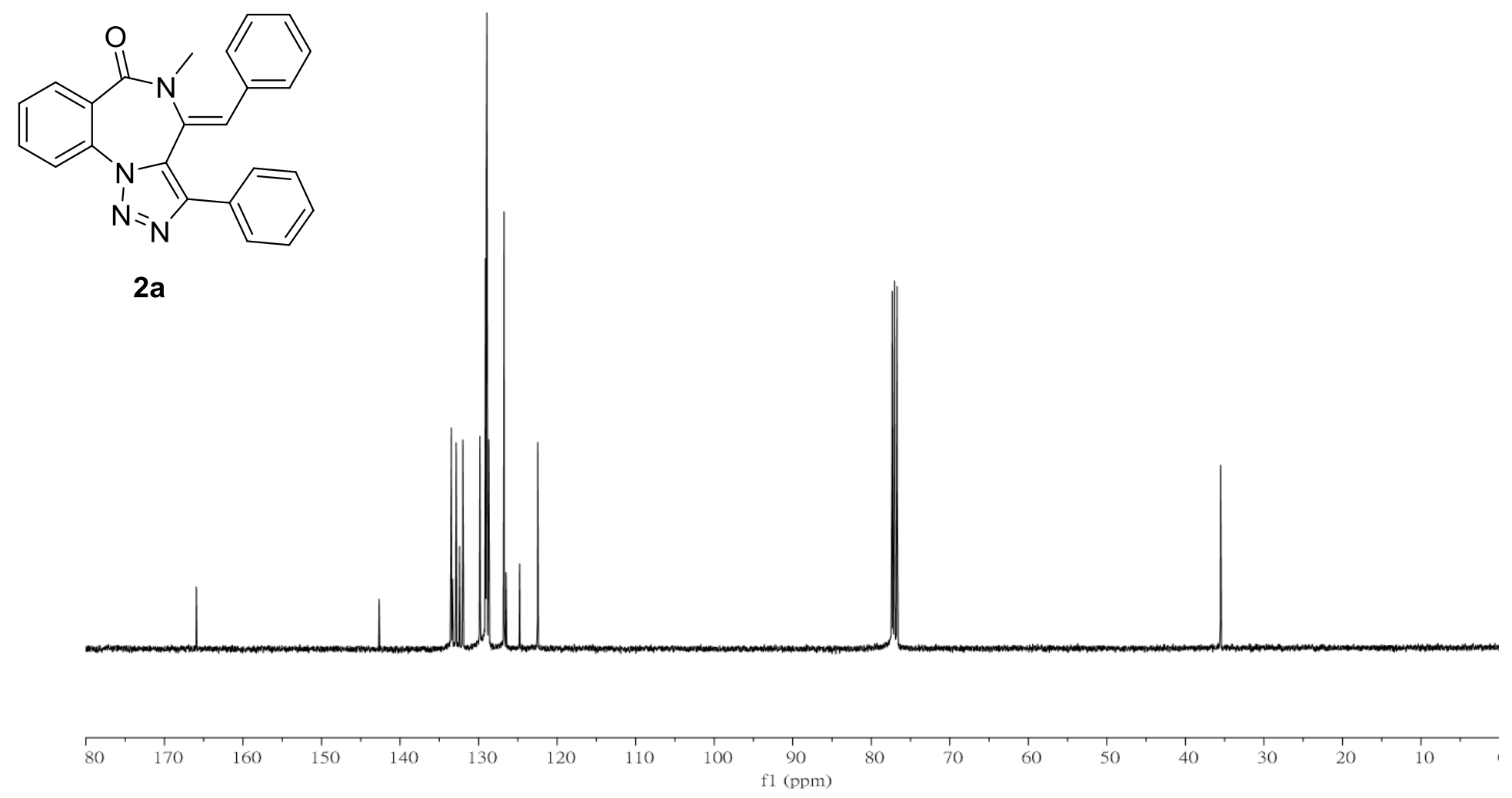

${ }^{13} \mathrm{C}$ NMR Spectrum (101 MHz) of compound 2a in $\mathrm{CDCl}_{3}$ 

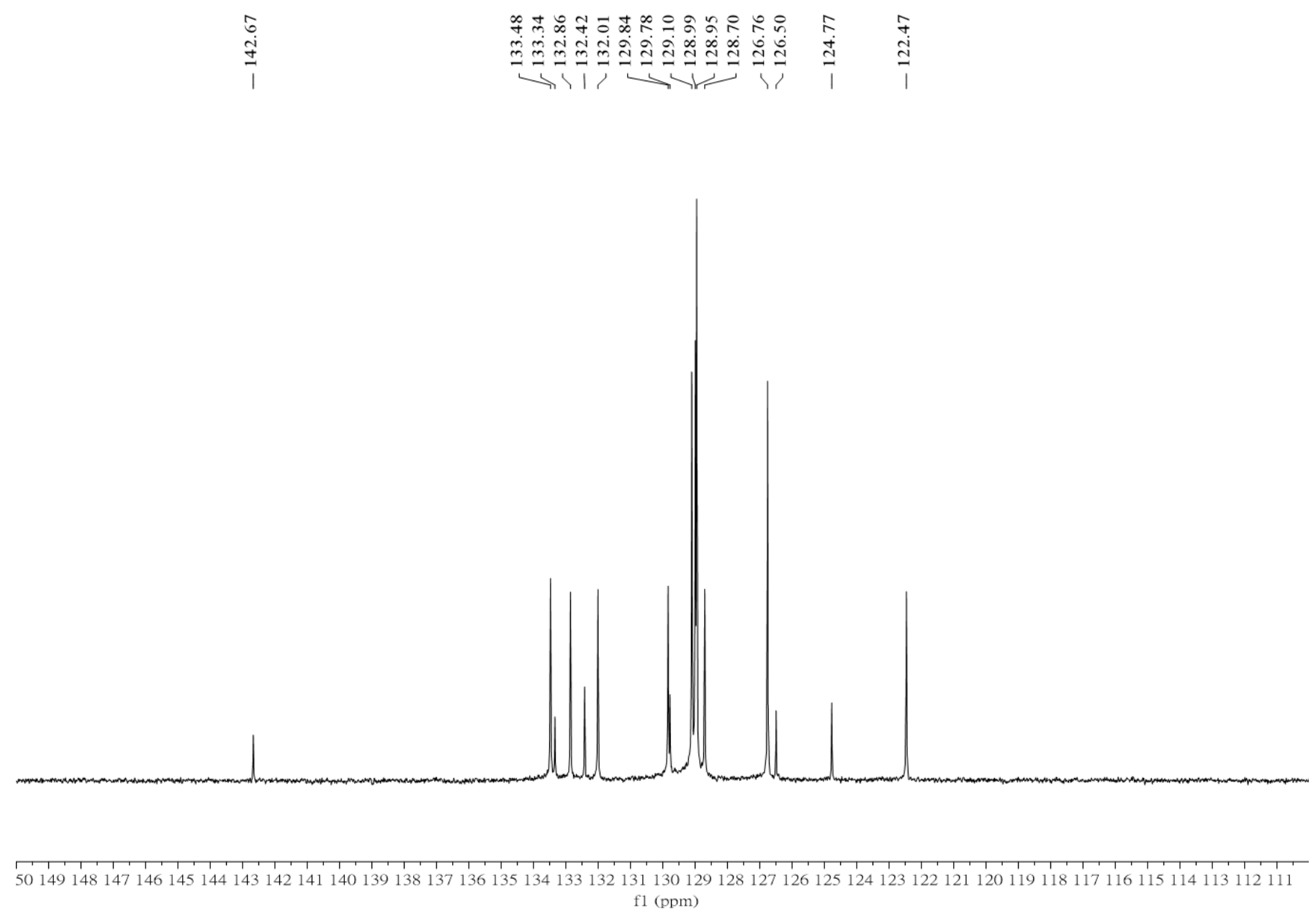

Expansion of ${ }^{13} \mathrm{C}$ NMR Spectrum (101 MHz) of compound 2a in $\mathrm{CDCl}_{3}$ 


\section{Display Report}

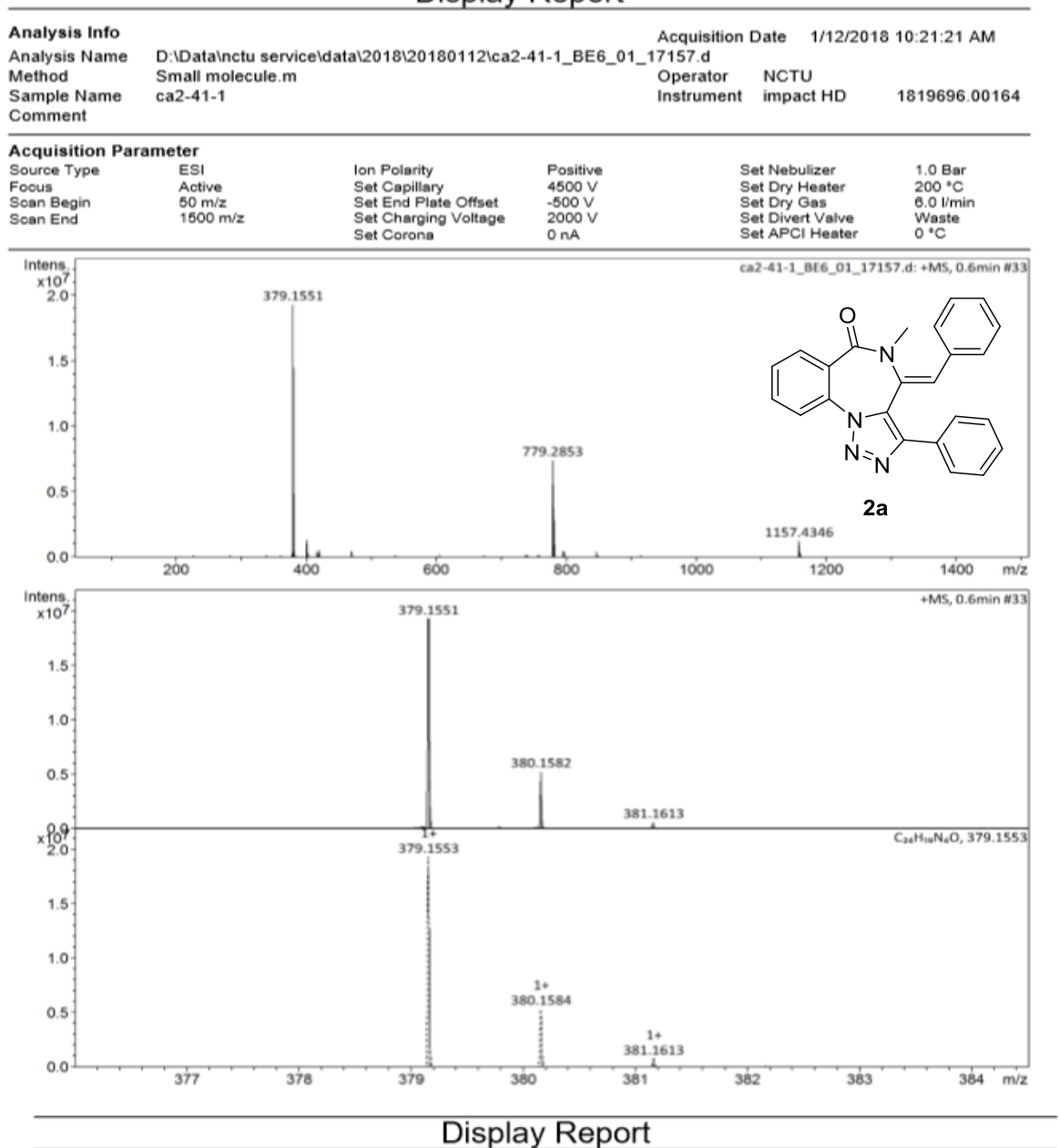

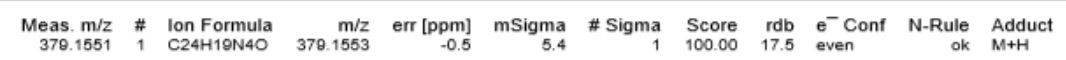

HRMS of compound 2a 


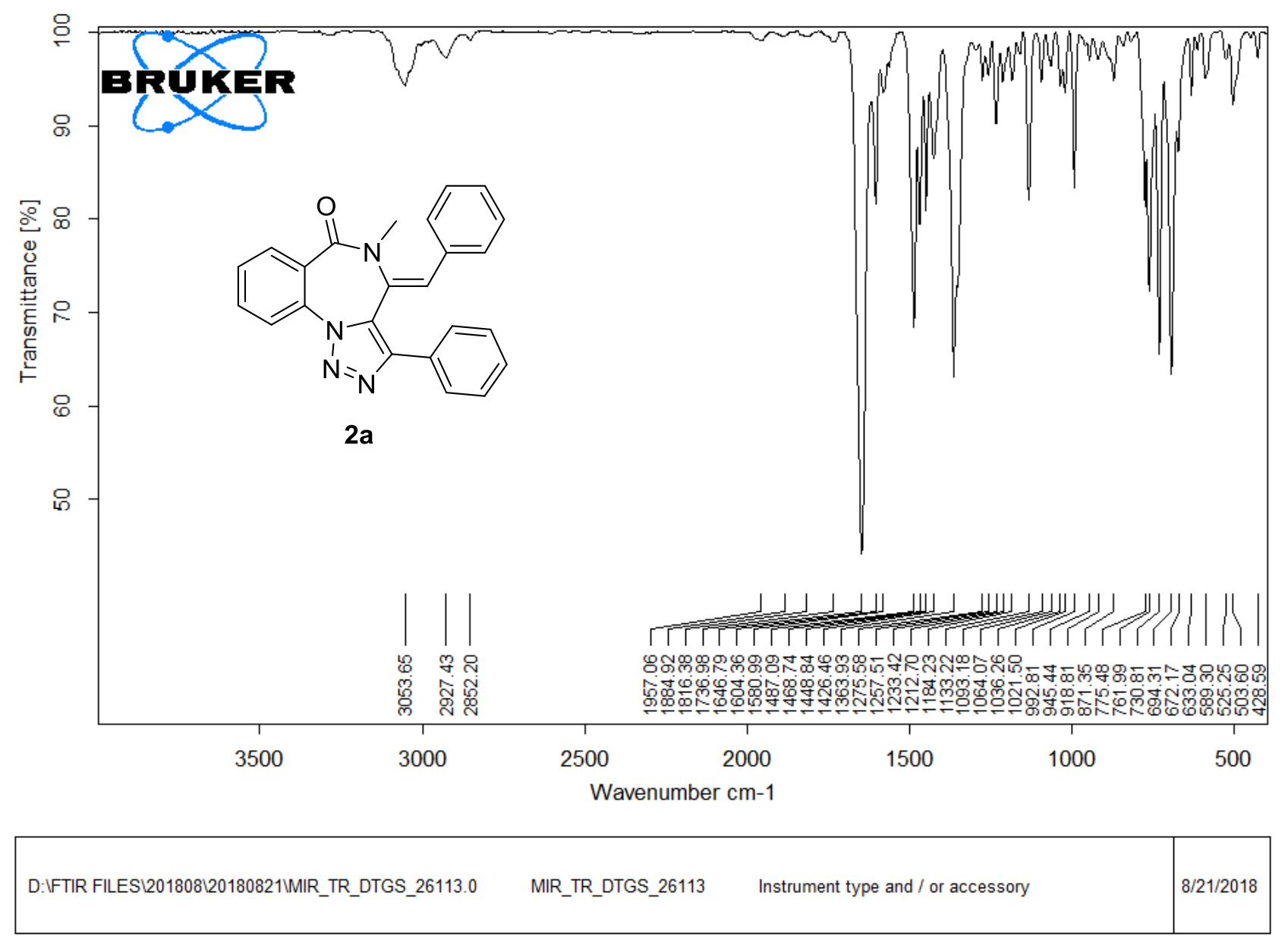

Page 1/1

IR of compound $\mathbf{2 a}$ 


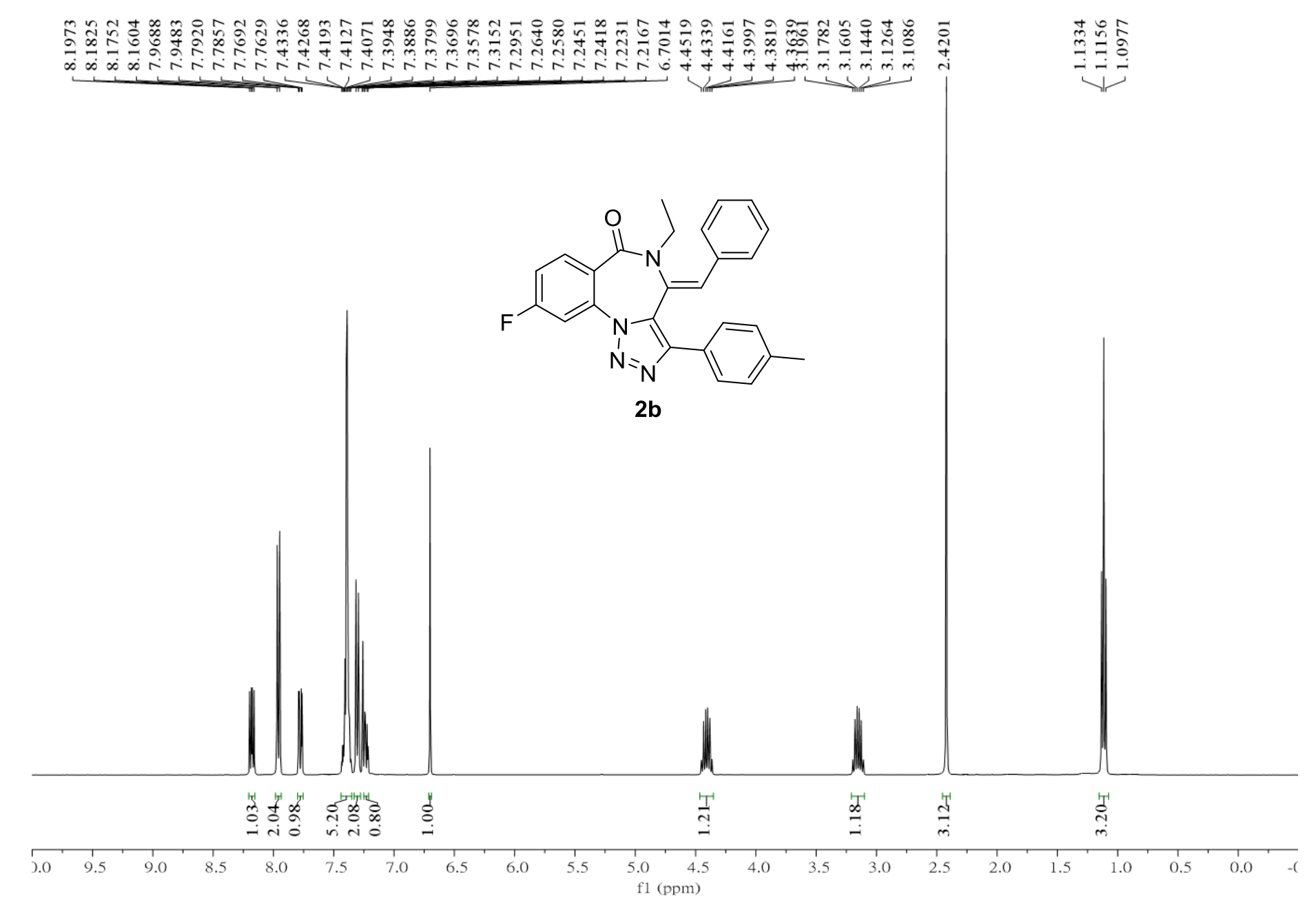

${ }^{1} \mathrm{H}$ NMR Spectrum (400 MHz) of compound $\mathbf{2} \mathbf{b}$ in $\mathrm{CDCl}_{3}$ 


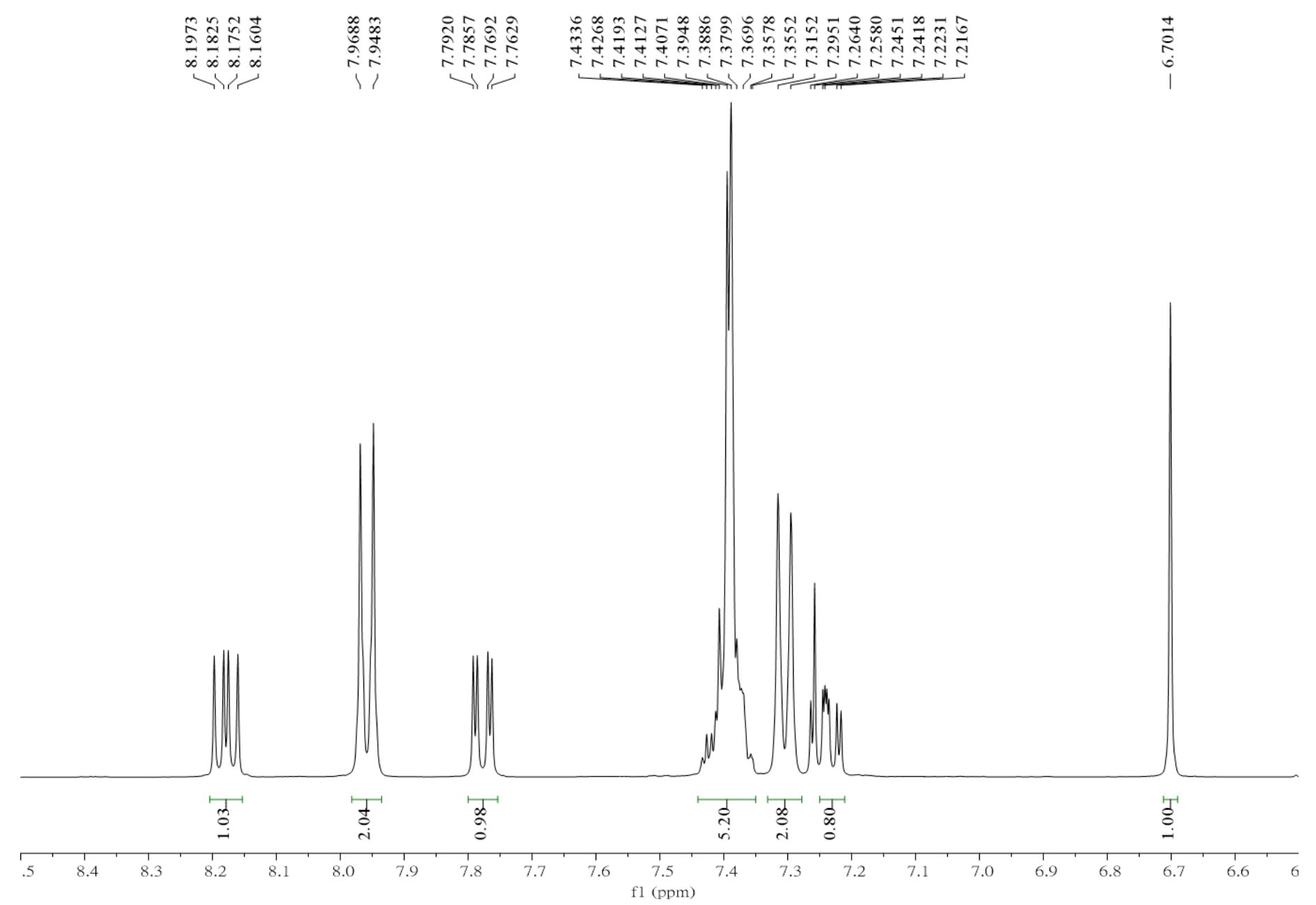

Expansion of ${ }^{1} \mathrm{H}$ NMR Spectrum (400 MHz) of compound $\mathbf{2 b}$ in $\mathrm{CDCl}_{3}$ 


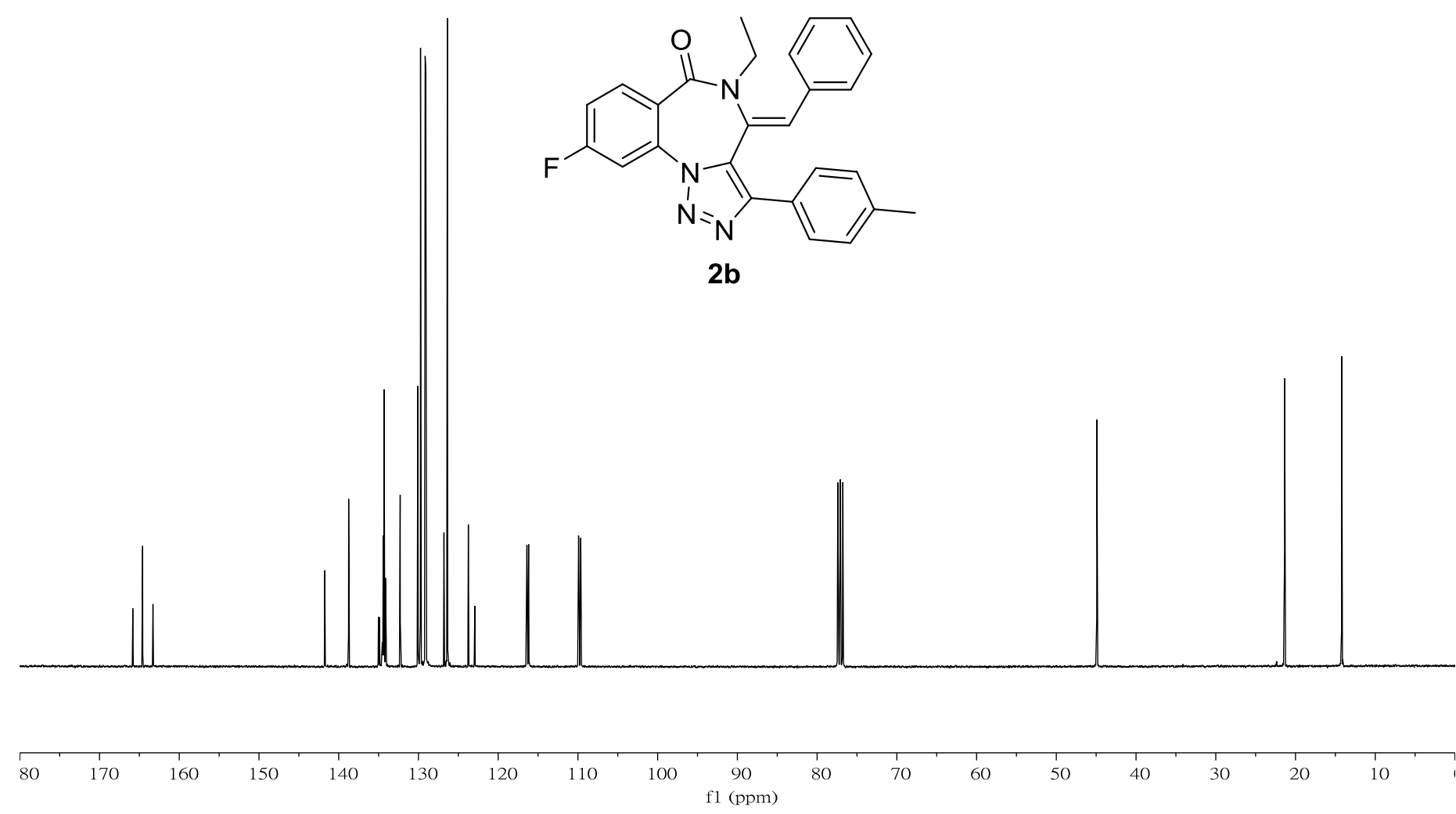

${ }^{13} \mathrm{C}$ NMR Spectrum (101 MHz) of compound $\mathbf{2 b}$ in $\mathrm{CDCl}_{3}$ 


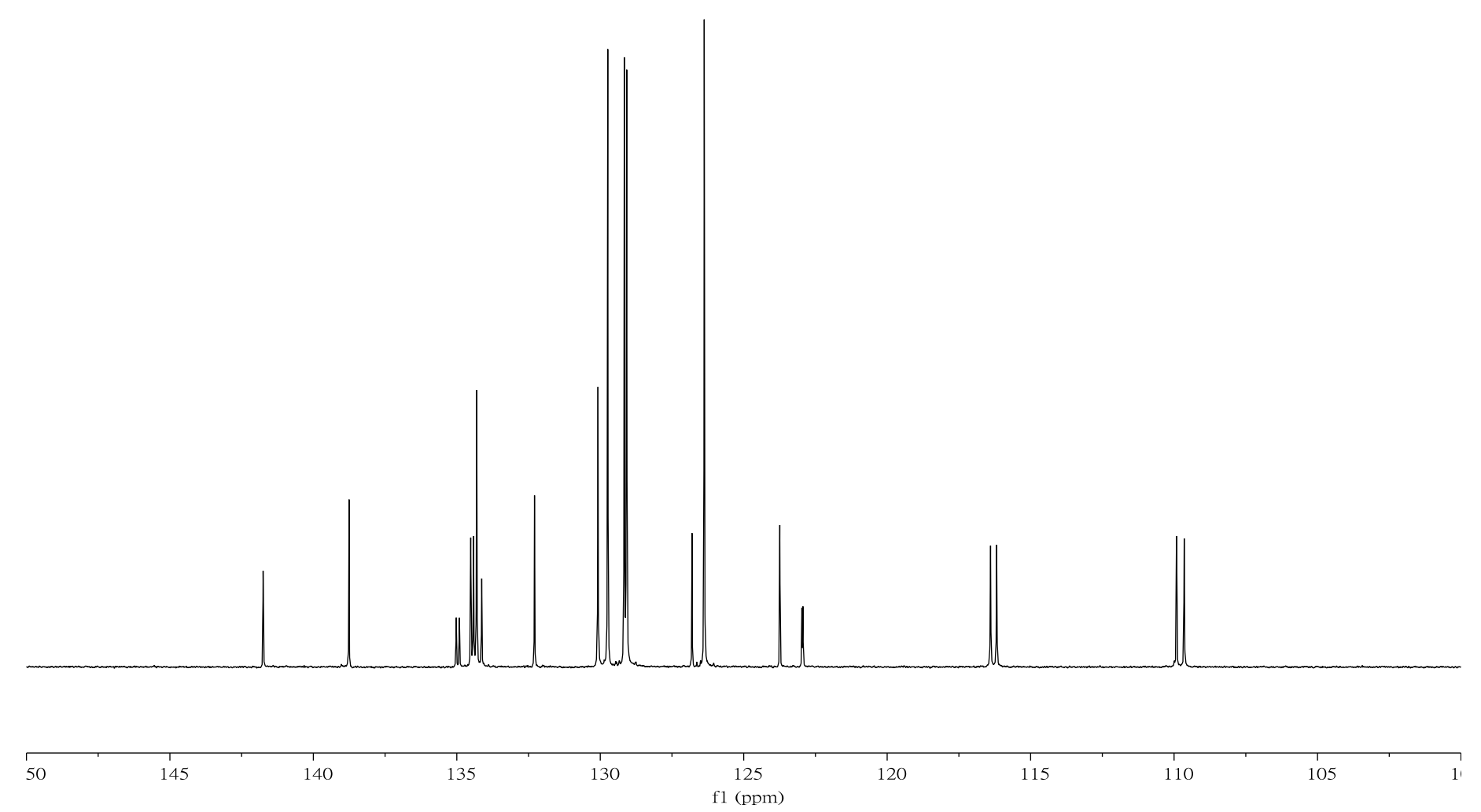

Expansion of ${ }^{13} \mathrm{C}$ NMR Spectrum $(101 \mathrm{MHz})$ of compound $\mathbf{2 b}$ in $\mathrm{CDCl}_{3}$ 


\section{Display Report}

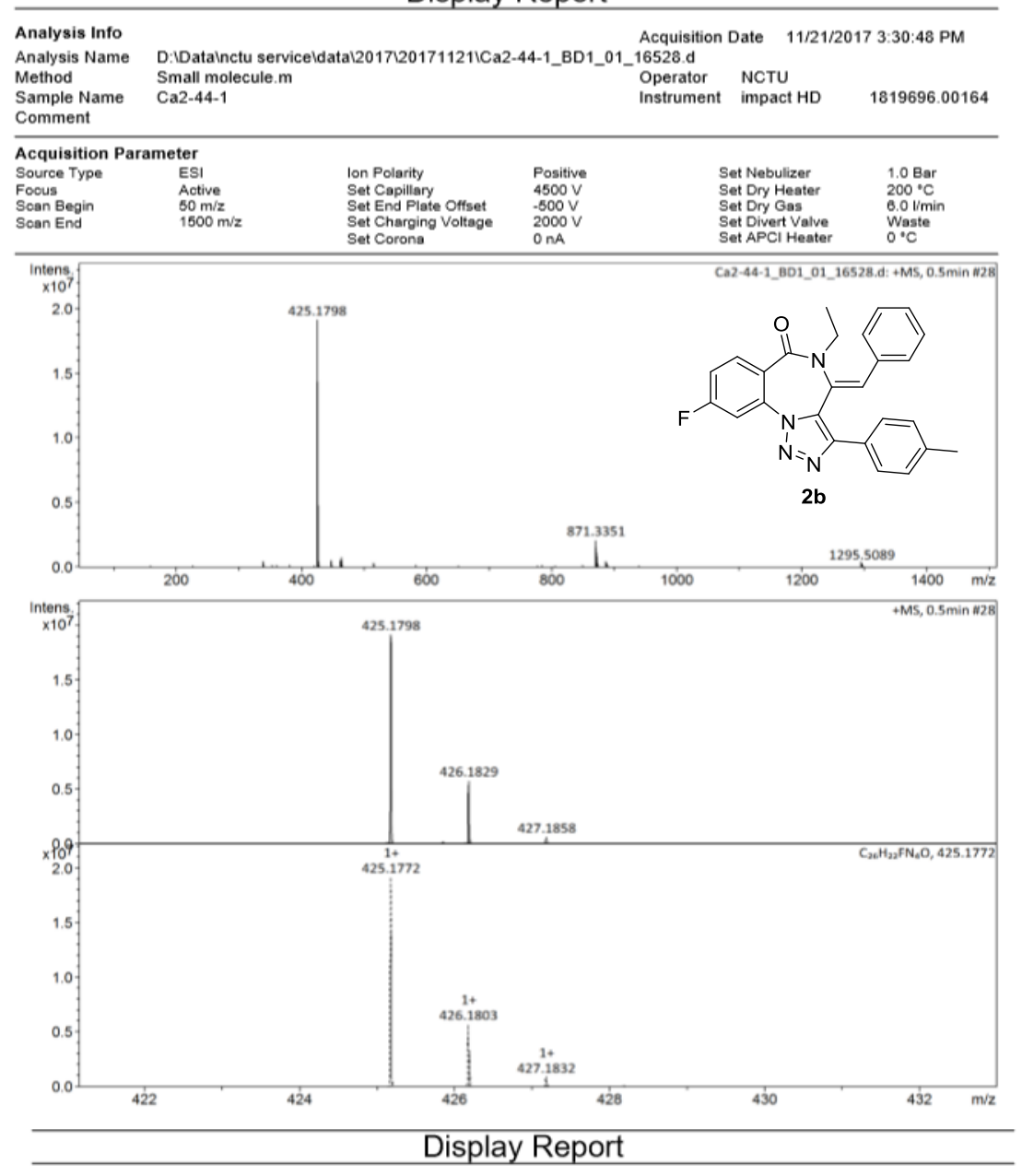

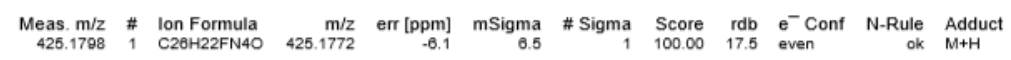

HRMS of compound $\mathbf{2 b}$ 


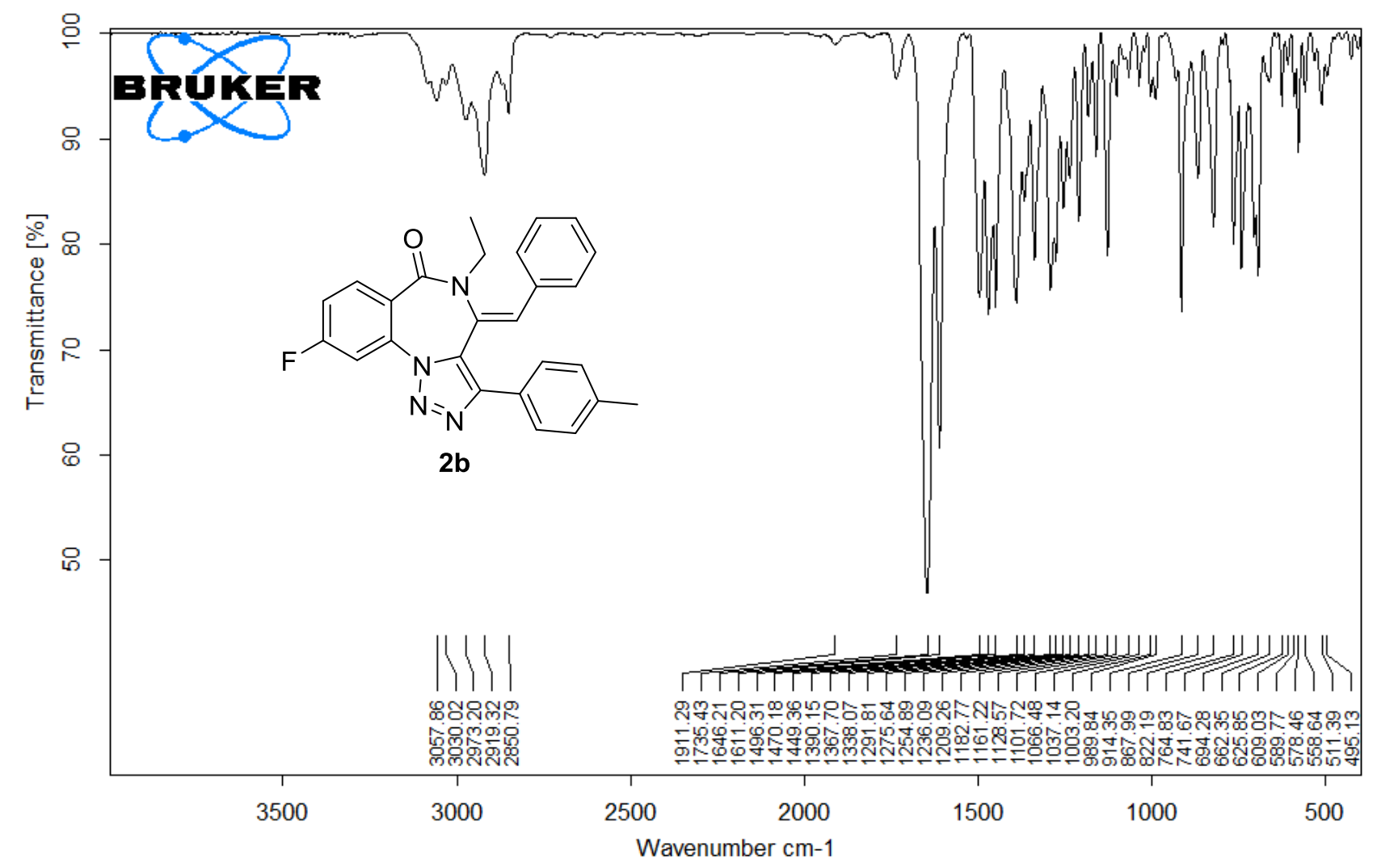

\begin{tabular}{|ll|l|l|}
\hline D:IFTIR FILESI201808120180821MIR_TR_DTGS_26114.0 $\quad$ MIR_TR_DTGS_26114 & Instrument type and / or accessory & $8 / 21 / 2018$ \\
\hline
\end{tabular}

Page 1/1

IR of compound $\mathbf{2 b}$ 


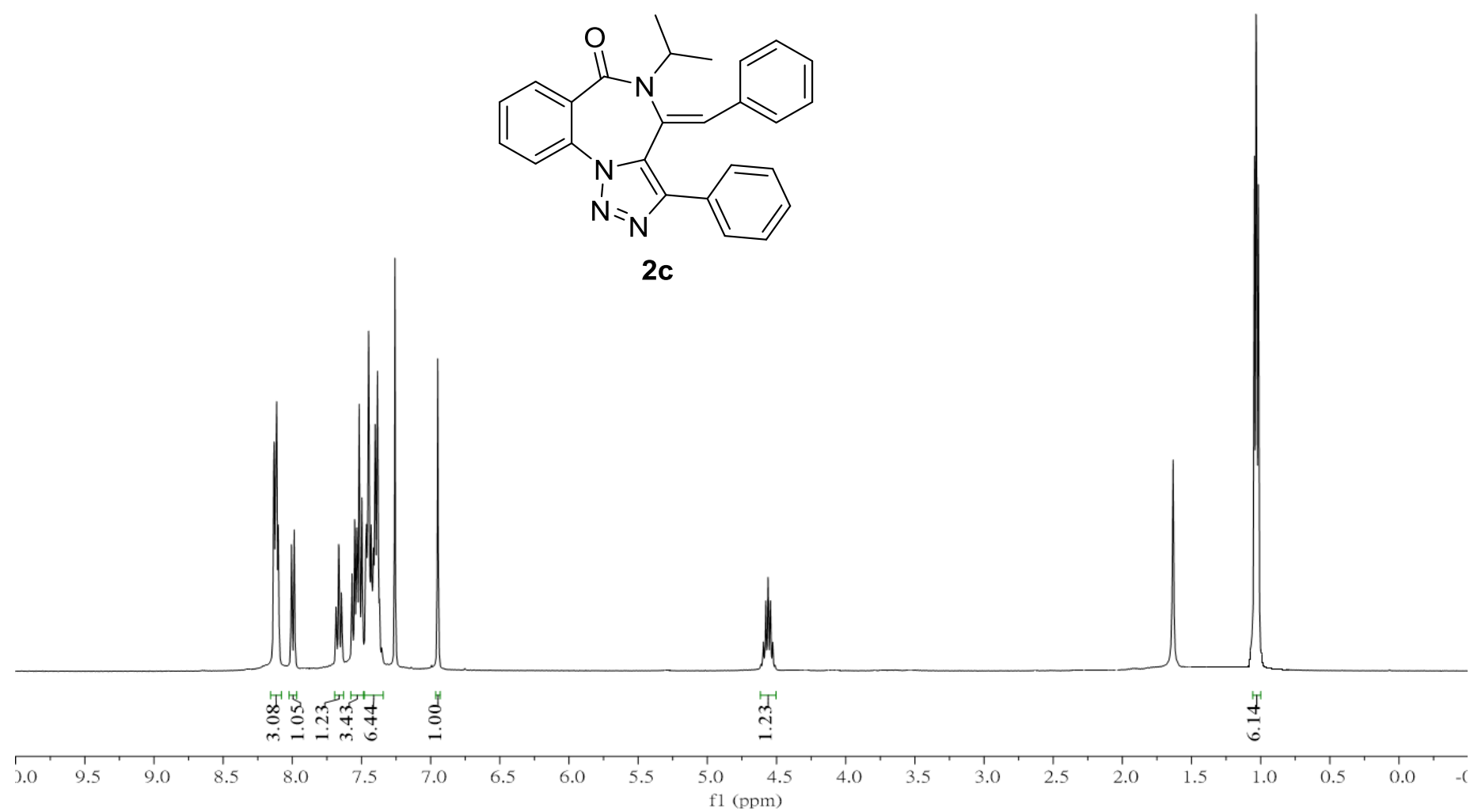

${ }^{1} \mathrm{H}$ NMR Spectrum (400 MHz) of compound $2 \mathbf{c}$ in $\mathrm{CDCl}_{3}$ 

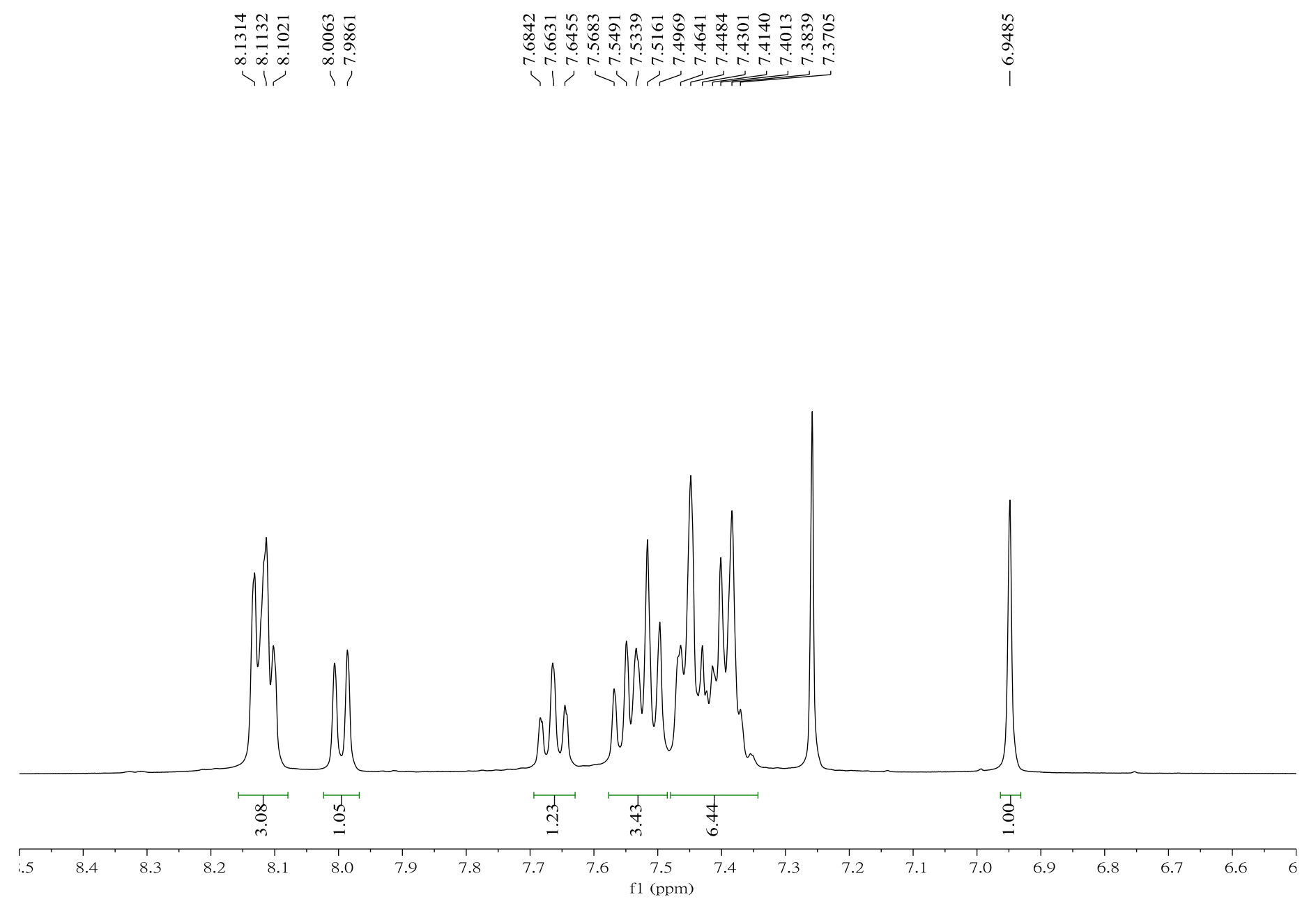

Expansion of ${ }^{1} \mathrm{H}$ NMR Spectrum $\left(400 \mathrm{MHz}\right.$ ) of compound $2 \mathrm{c}$ in $\mathrm{CDCl}_{3}$ 


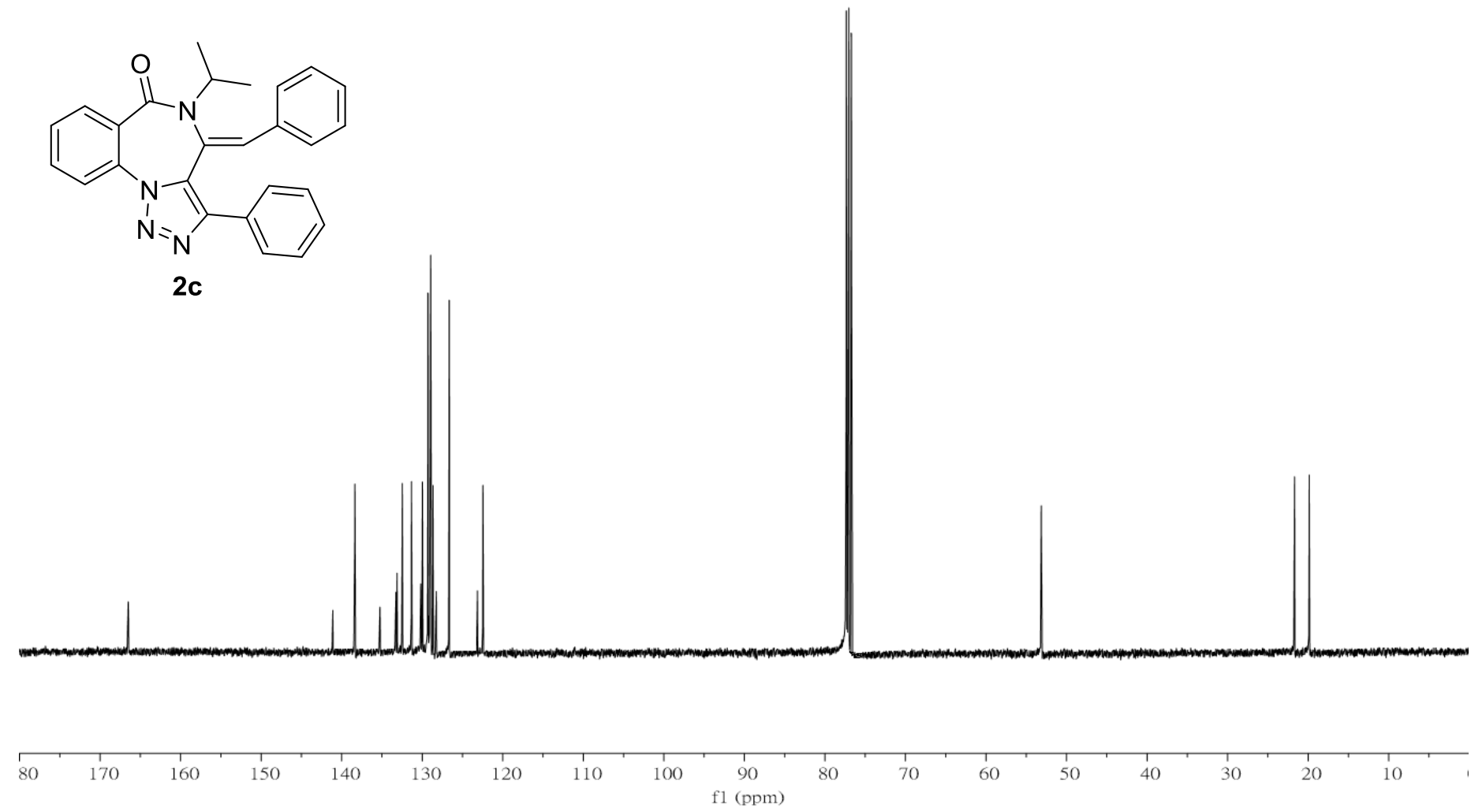

${ }^{13} \mathrm{C}$ NMR Spectrum (101 MHz) of compound $\mathbf{2 c}$ in $\mathrm{CDCl}_{3}$ 

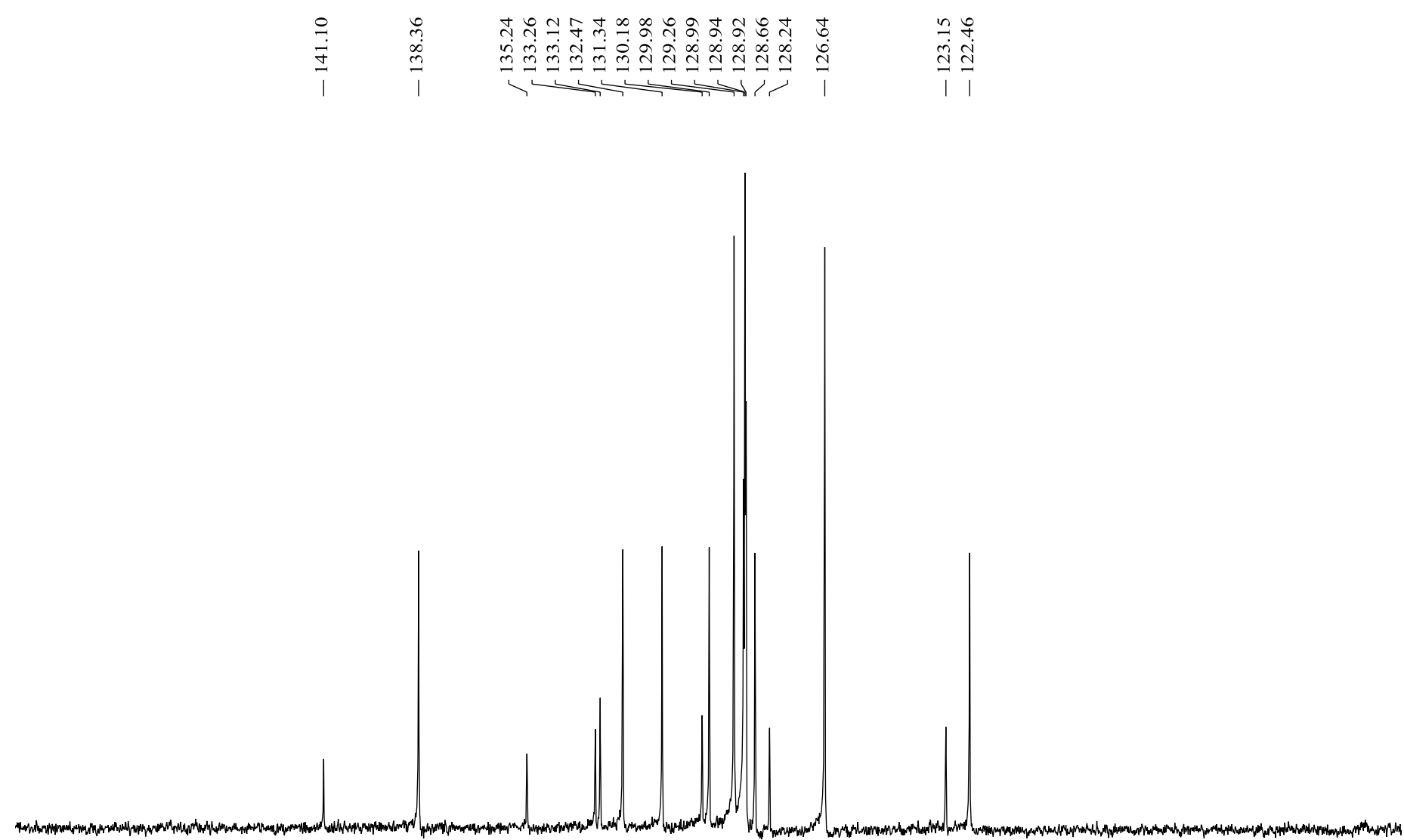

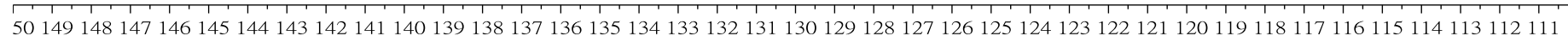
f1 (ppm)

Expansion of ${ }^{13} \mathrm{C}$ NMR Spectrum $(101 \mathrm{MHz})$ of compound $2 \mathrm{c}$ in $\mathrm{CDCl}_{3}$ 
Display Report

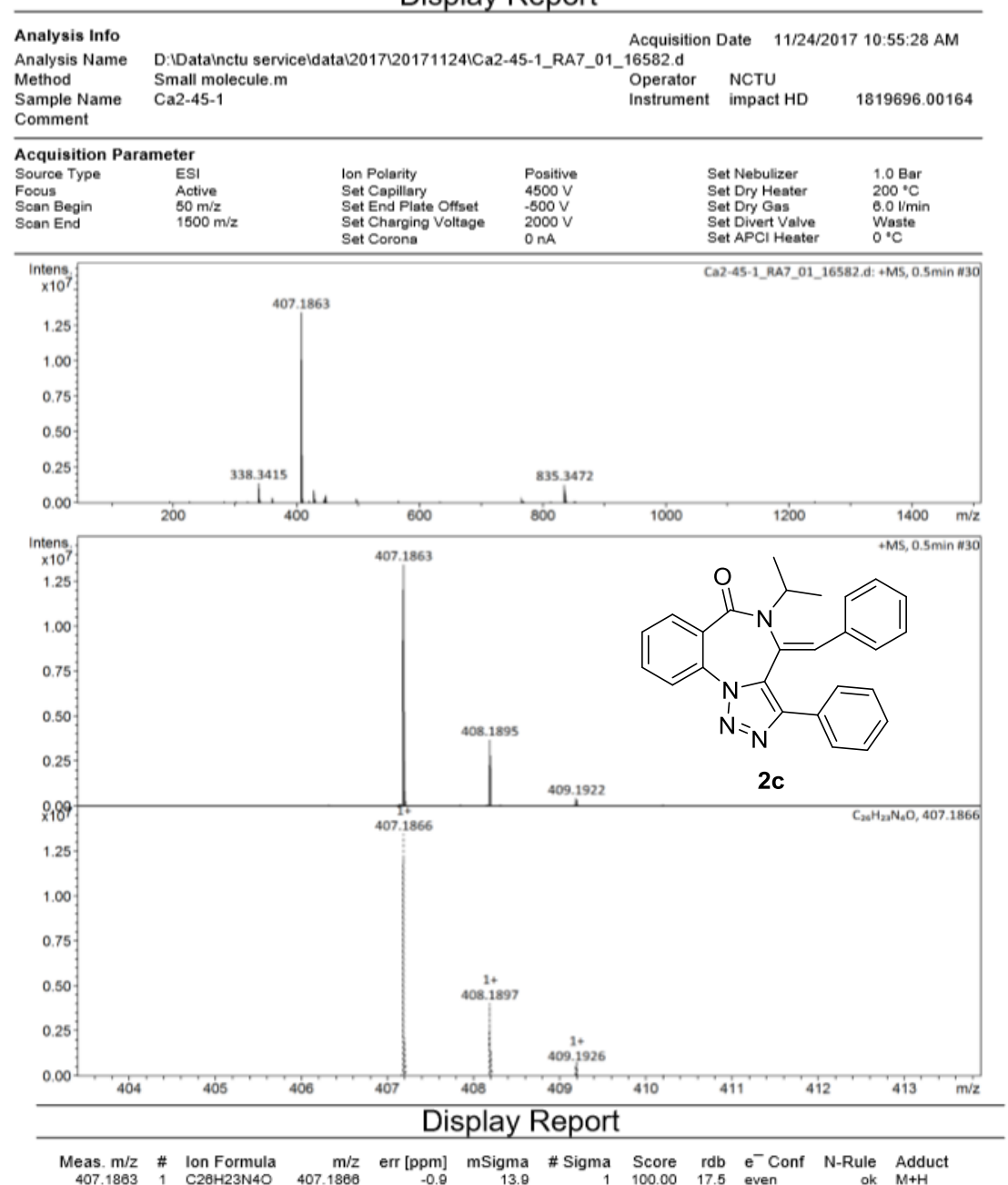

HRMS of compound 2c 


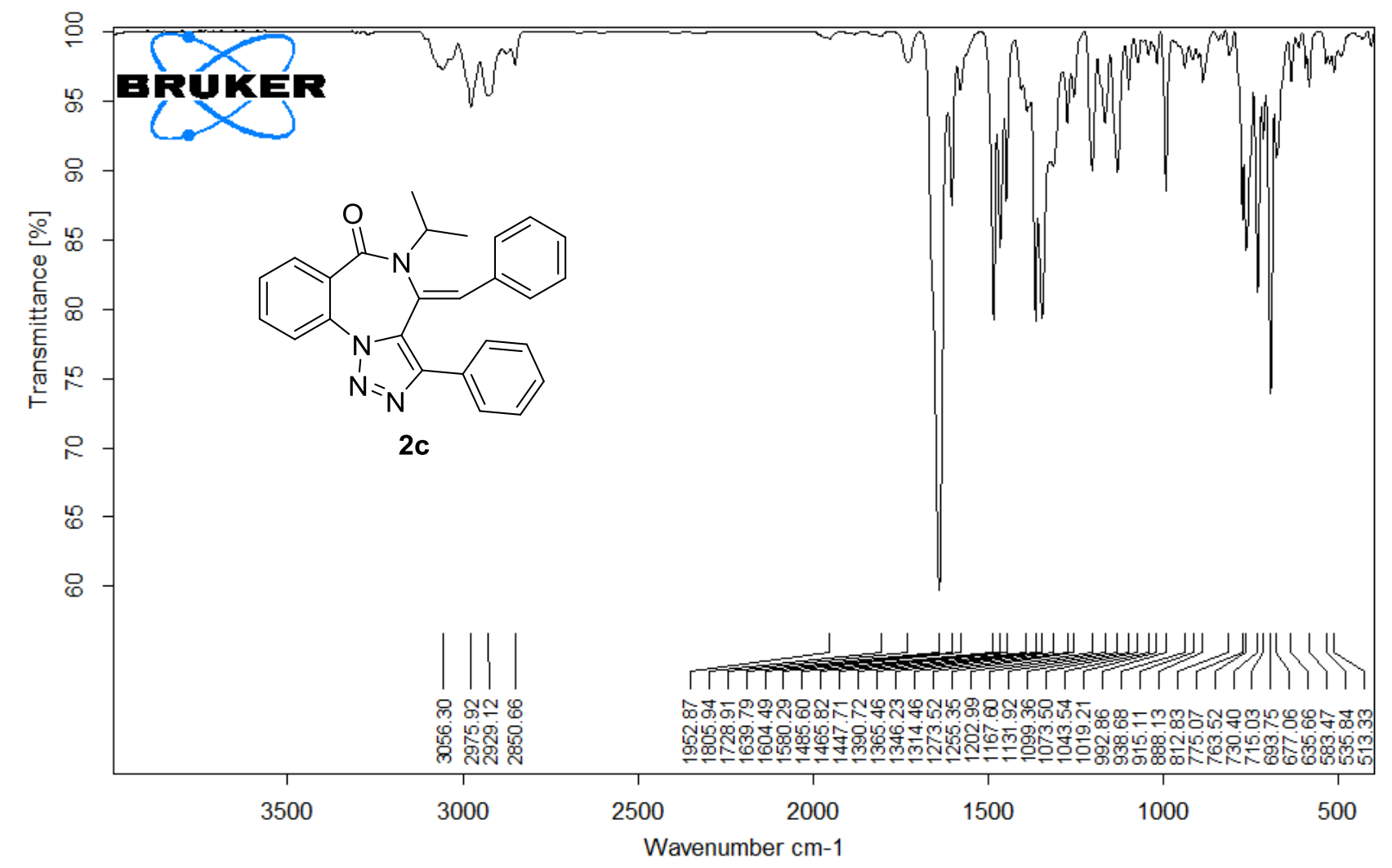

\begin{tabular}{|lcl|l|}
\hline D:IFTIR FILESI201808120180821IMIR_TR_DTGS_26115.0 $\quad$ MIR_TR_DTGS_26115 $\quad$ Instrument type and / or accessory & $8 / 21 / 2018$ \\
\hline
\end{tabular}

Page 1/1

IR of compound $\mathbf{2 c}$ 


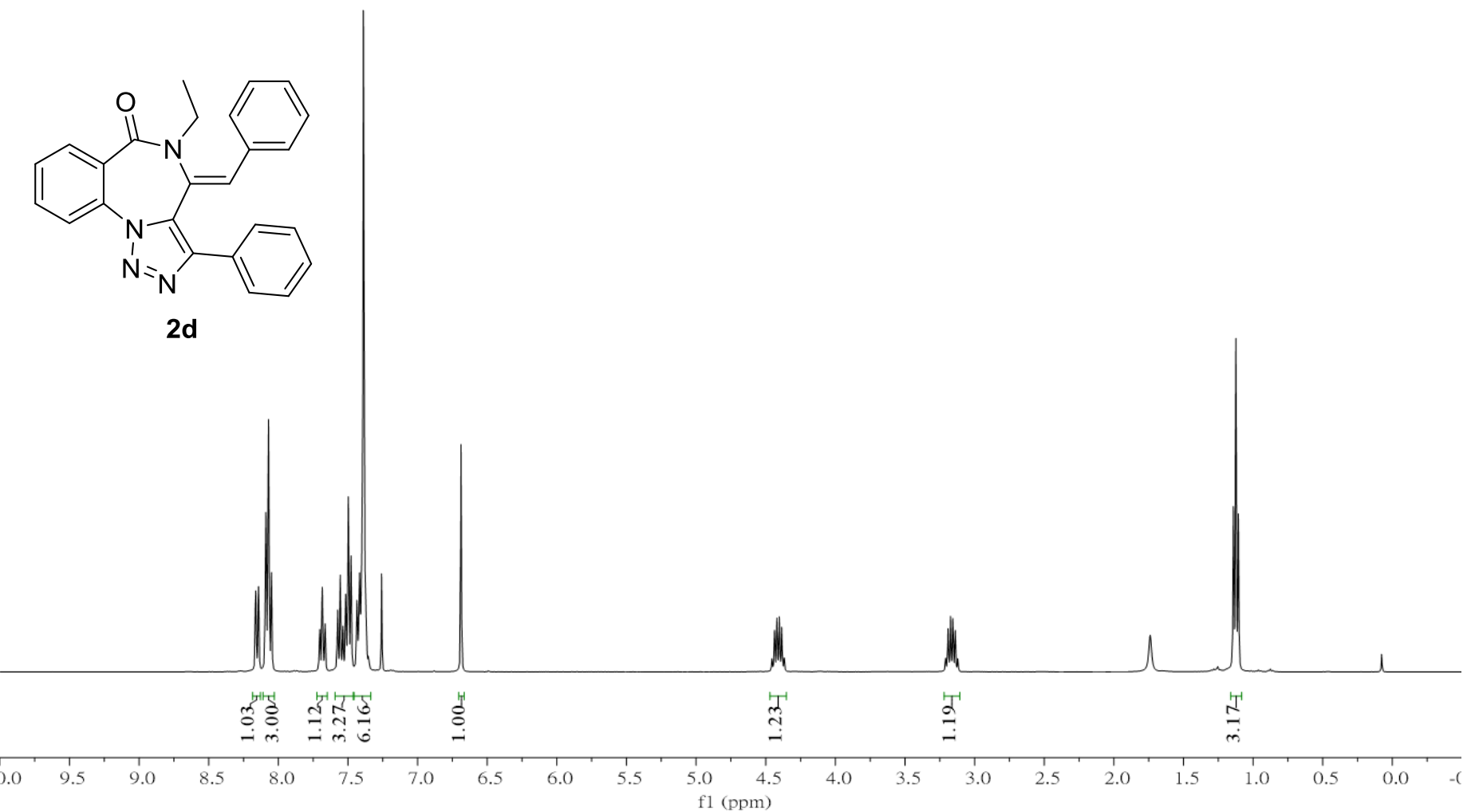

${ }^{1} \mathrm{H}$ NMR Spectrum $\left(400 \mathrm{MHz}\right.$ ) of compound $\mathbf{2 d}$ in $\mathrm{CDCl}_{3}$ 


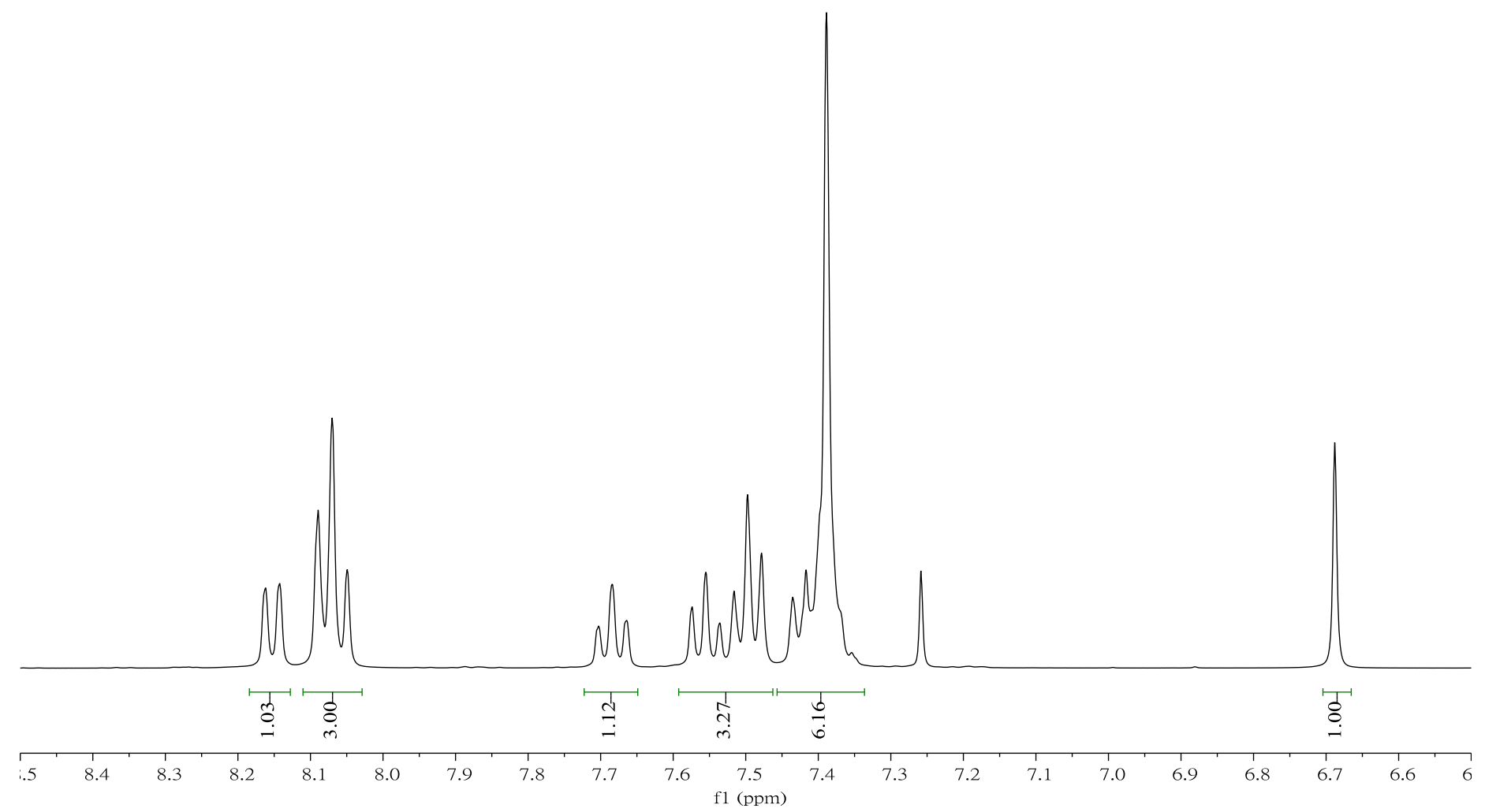

Expansion of ${ }^{1} \mathrm{H}$ NMR Spectrum (400 MHz) of compound 2d in $\mathrm{CDCl}_{3}$ 

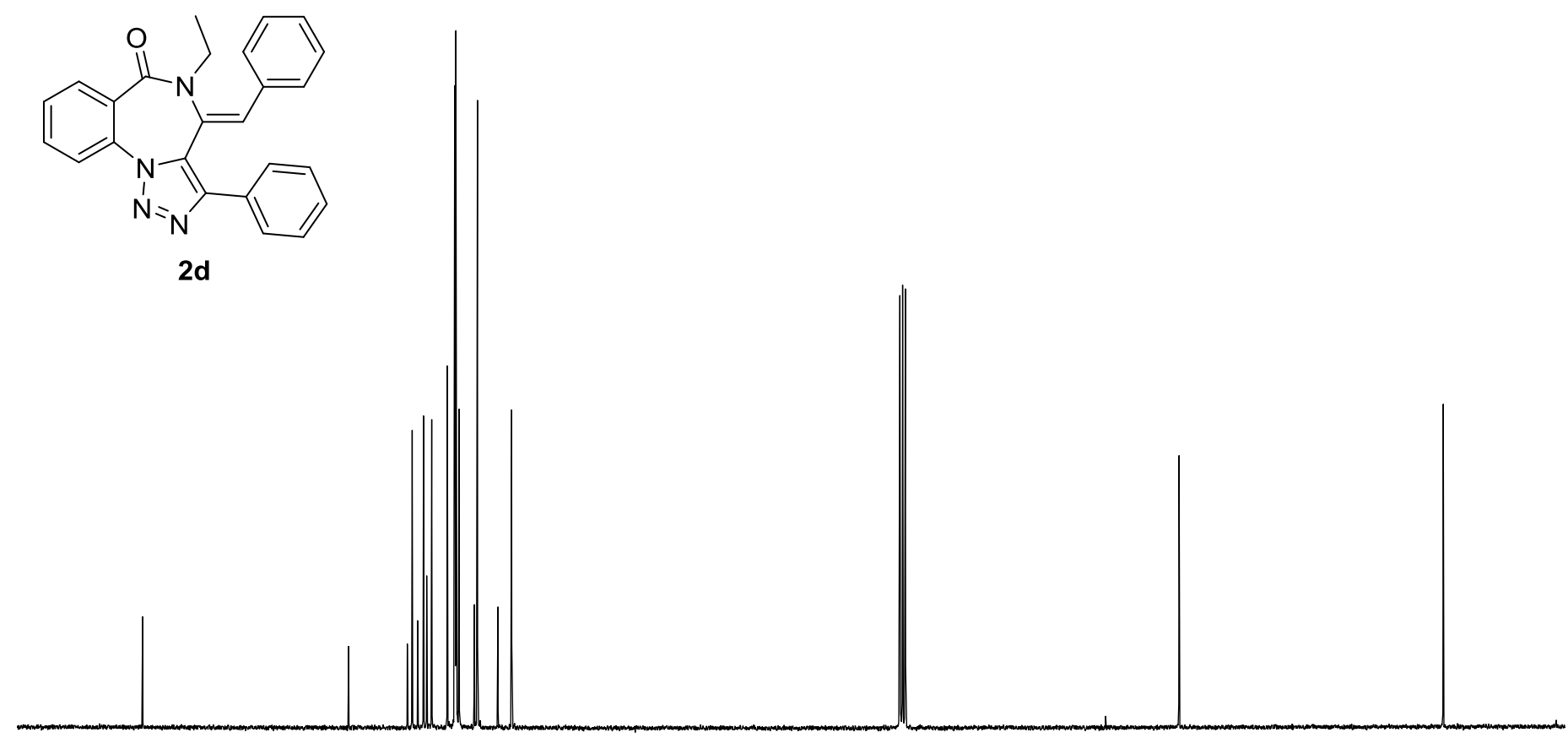

80

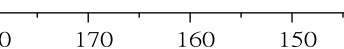

140 $130 \quad 120$ 110 100 90

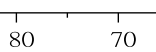

$60 \quad 50$

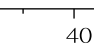

$30 \quad 20$

10

${ }^{13} \mathrm{C}$ NMR Spectrum (101 MHz) of compound $2 \mathbf{d}$ in $\mathrm{CDCl}_{3}$ 


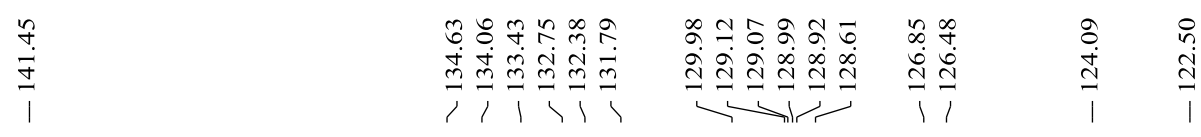

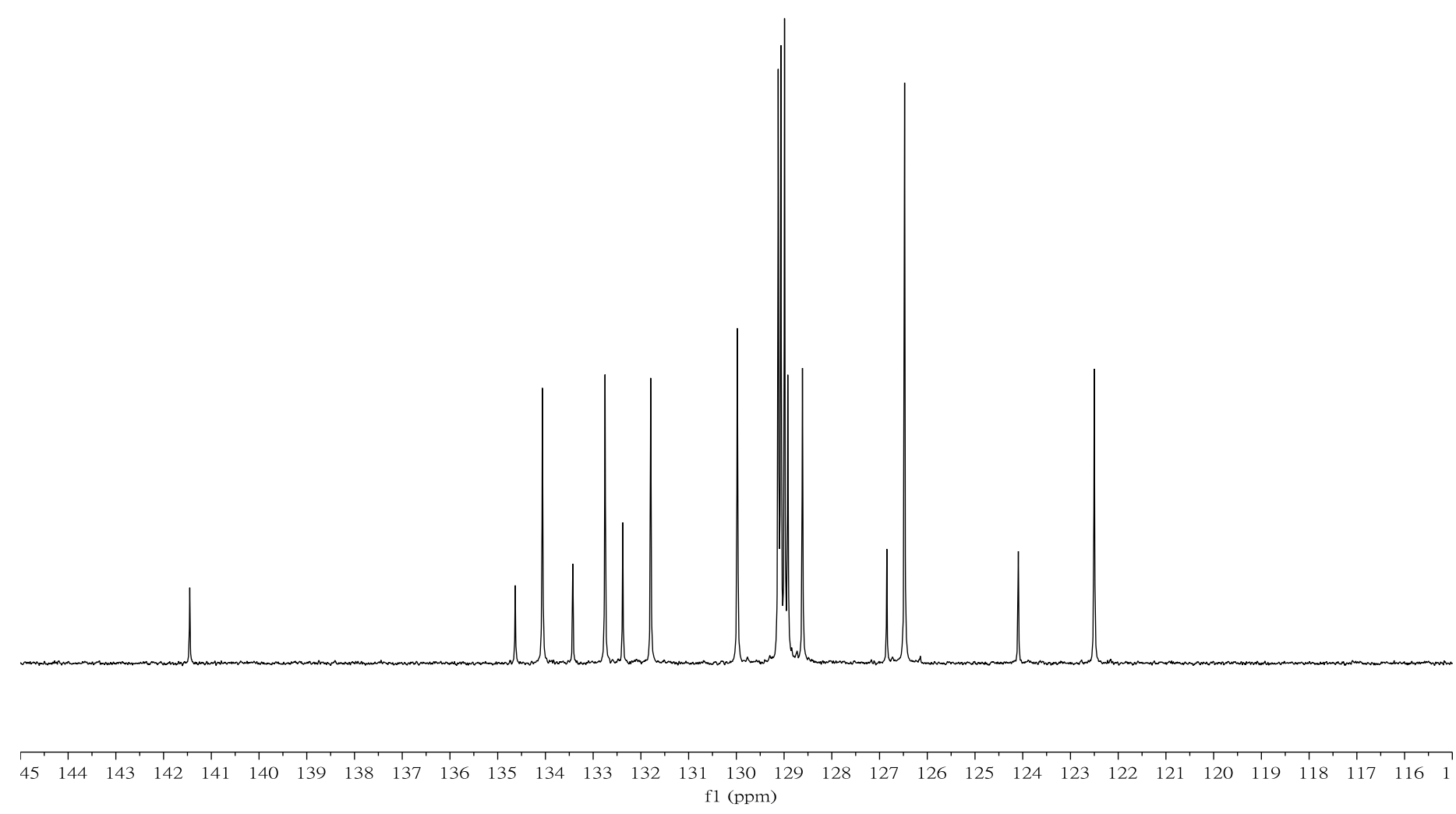

Expansion of ${ }^{13} \mathrm{C}$ NMR Spectrum $\left(101 \mathrm{MHz}\right.$ ) of compound 2d in $\mathrm{CDCl}_{3}$ 


\section{Display Report}

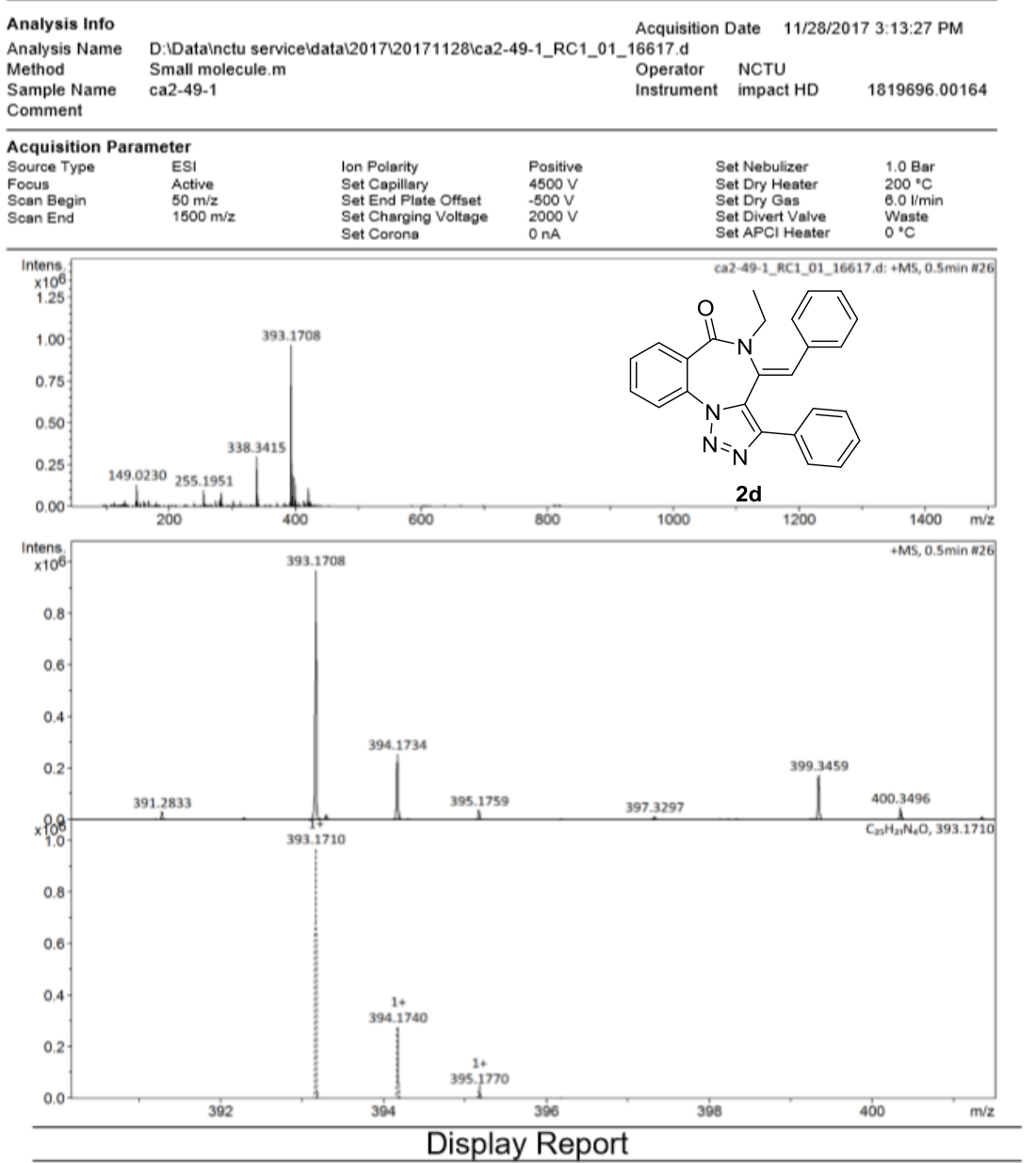

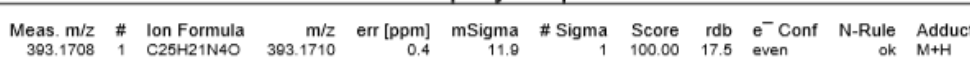

HRMS of compound 2d 


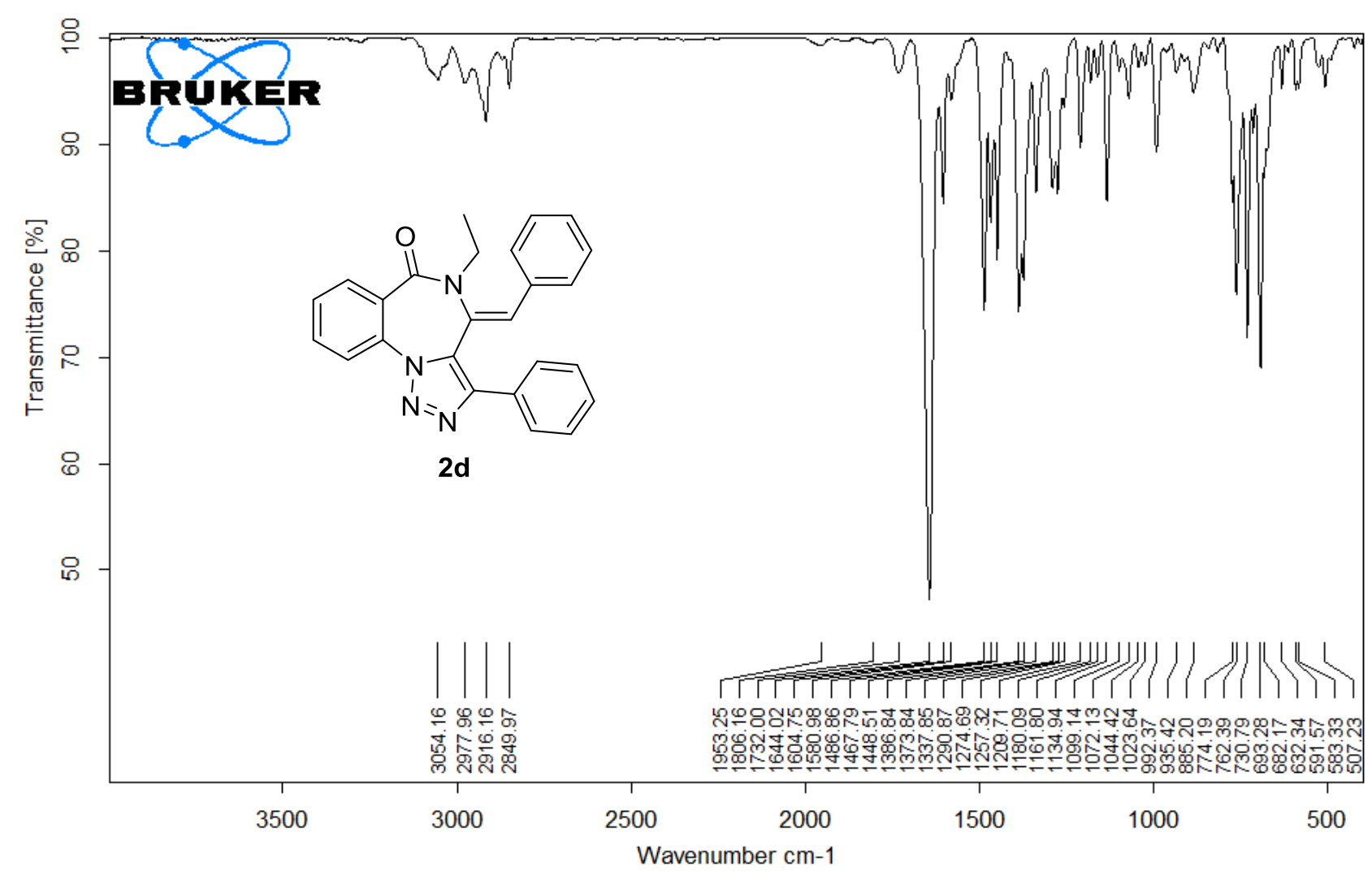

\begin{tabular}{|lcc|c|}
\hline D:IFTIR FILESI201808L20180821MIR_TR_DTGS_26116.0 MIR_TR_DTGS_26116 Instrument type and / or accessory & $8 / 21 / 2018$ \\
\hline
\end{tabular}

Page 1/1

IR of compound $\mathbf{2 d}$ 


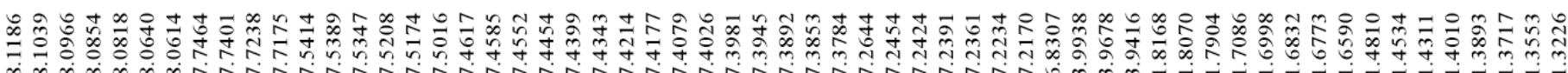 \\ Lind}

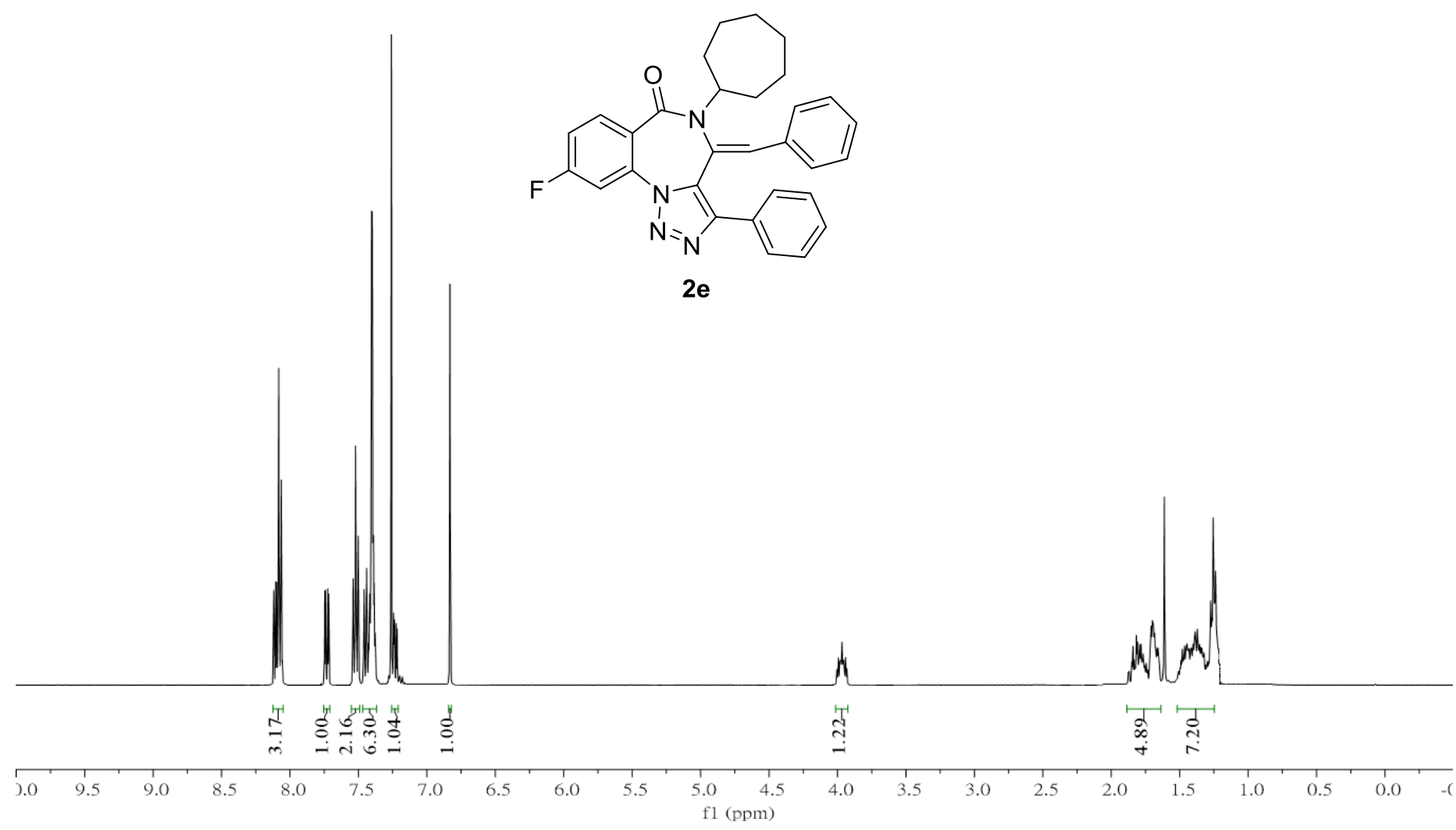

${ }^{1} \mathrm{H}$ NMR Spectrum (400 MHz) of compound $2 \mathbf{e}$ in $\mathrm{CDCl}_{3}$ 

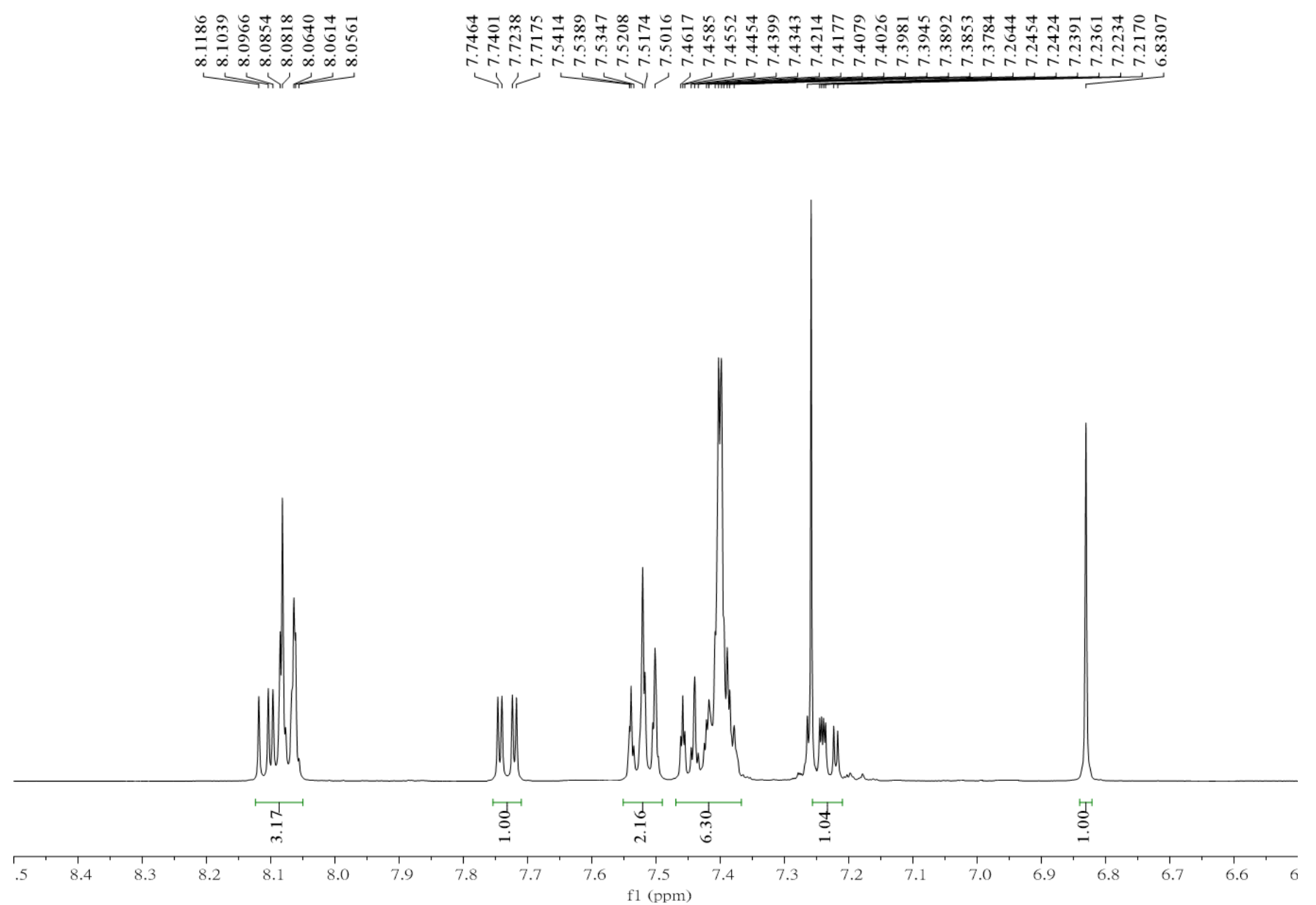

Expansion of ${ }^{1} \mathrm{H}$ NMR Spectrum (400 MHz) of compound $2 \mathbf{e}$ in $\mathrm{CDCl}_{3}$ 

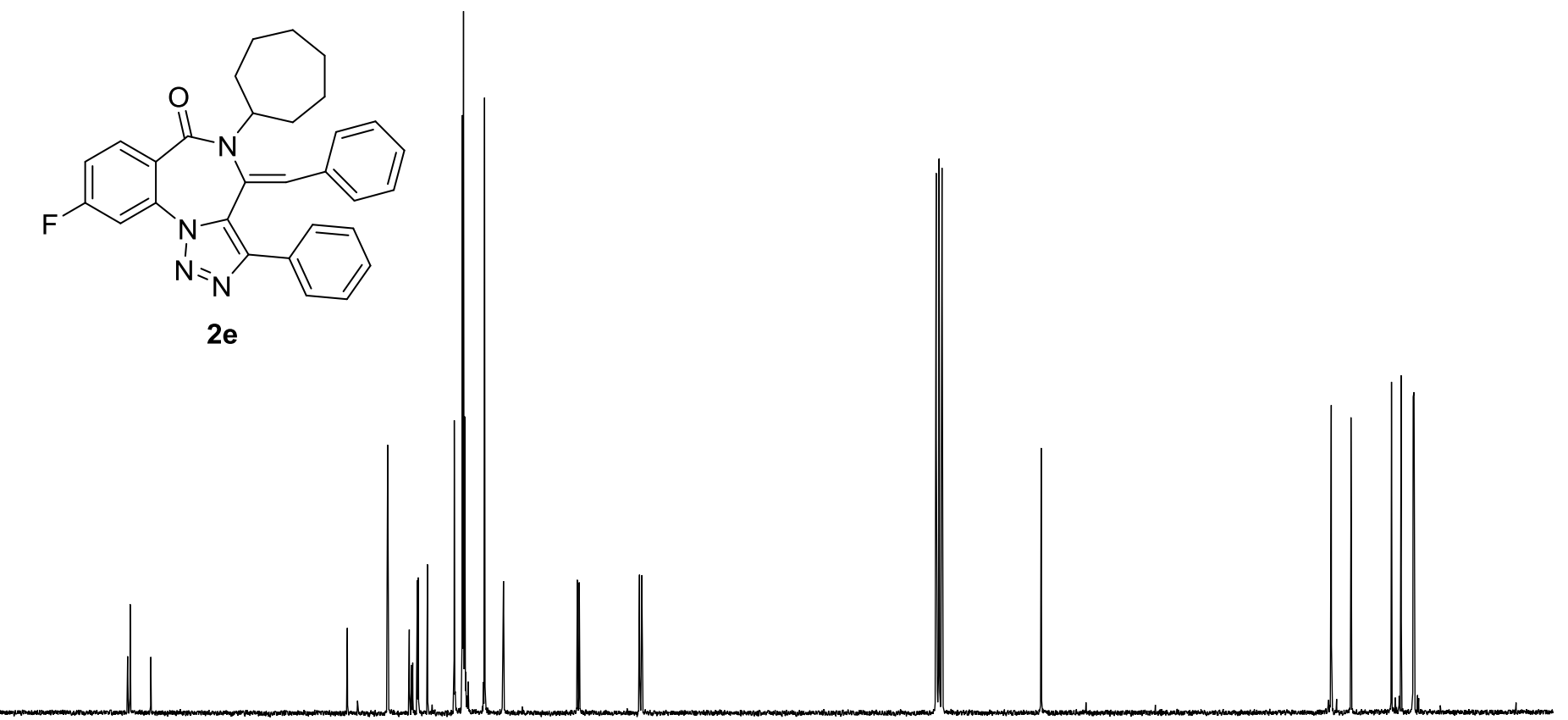

80
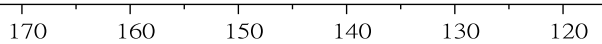

110

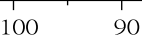

80

40

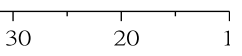

${ }^{13} \mathrm{C}$ NMR Spectrum (101 MHz) of compound $\mathbf{2 e}$ in $\mathrm{CDCl}_{3}$ 


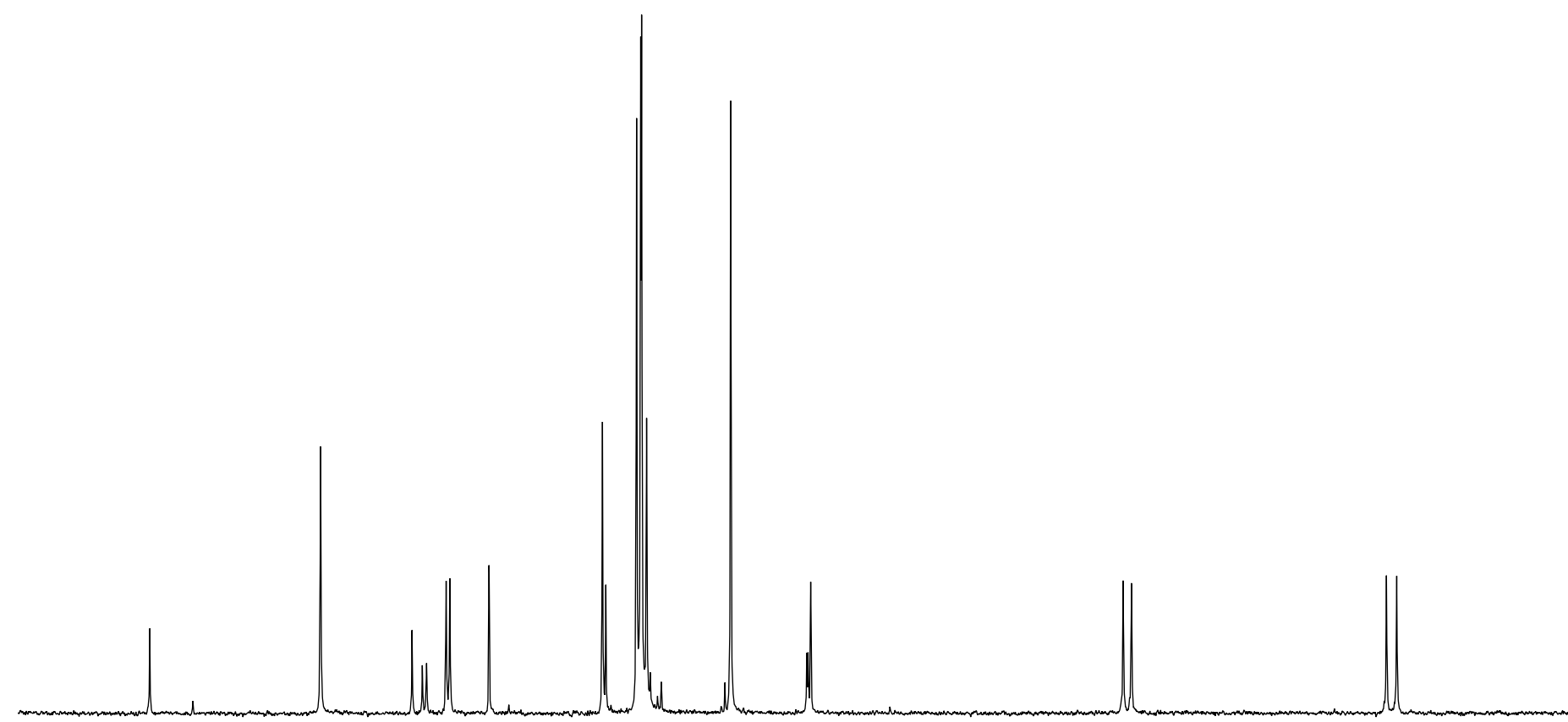

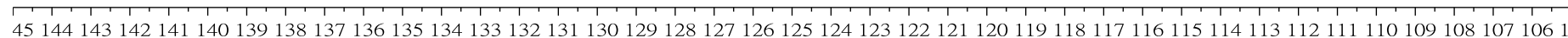
f1 (ppm)

Expansion of ${ }^{13} \mathrm{C}$ NMR Spectrum (101 MHz) of compound 2e in $\mathrm{CDCl}_{3}$ 
Display Report

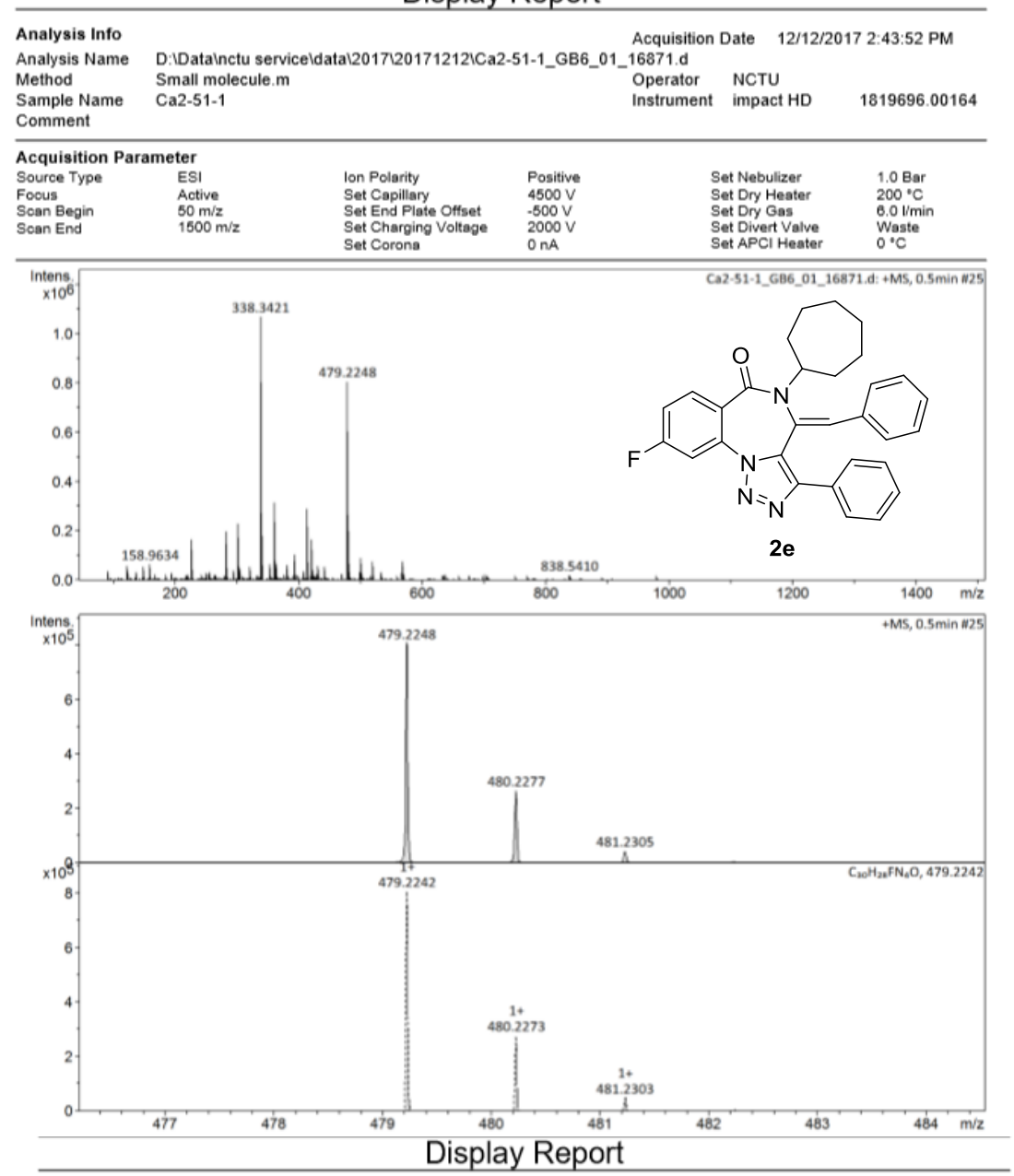

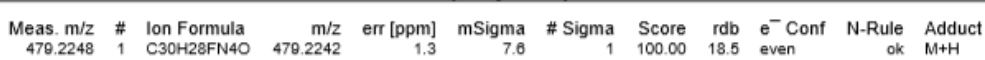

HRMS of compound $\mathbf{2 e}$ 


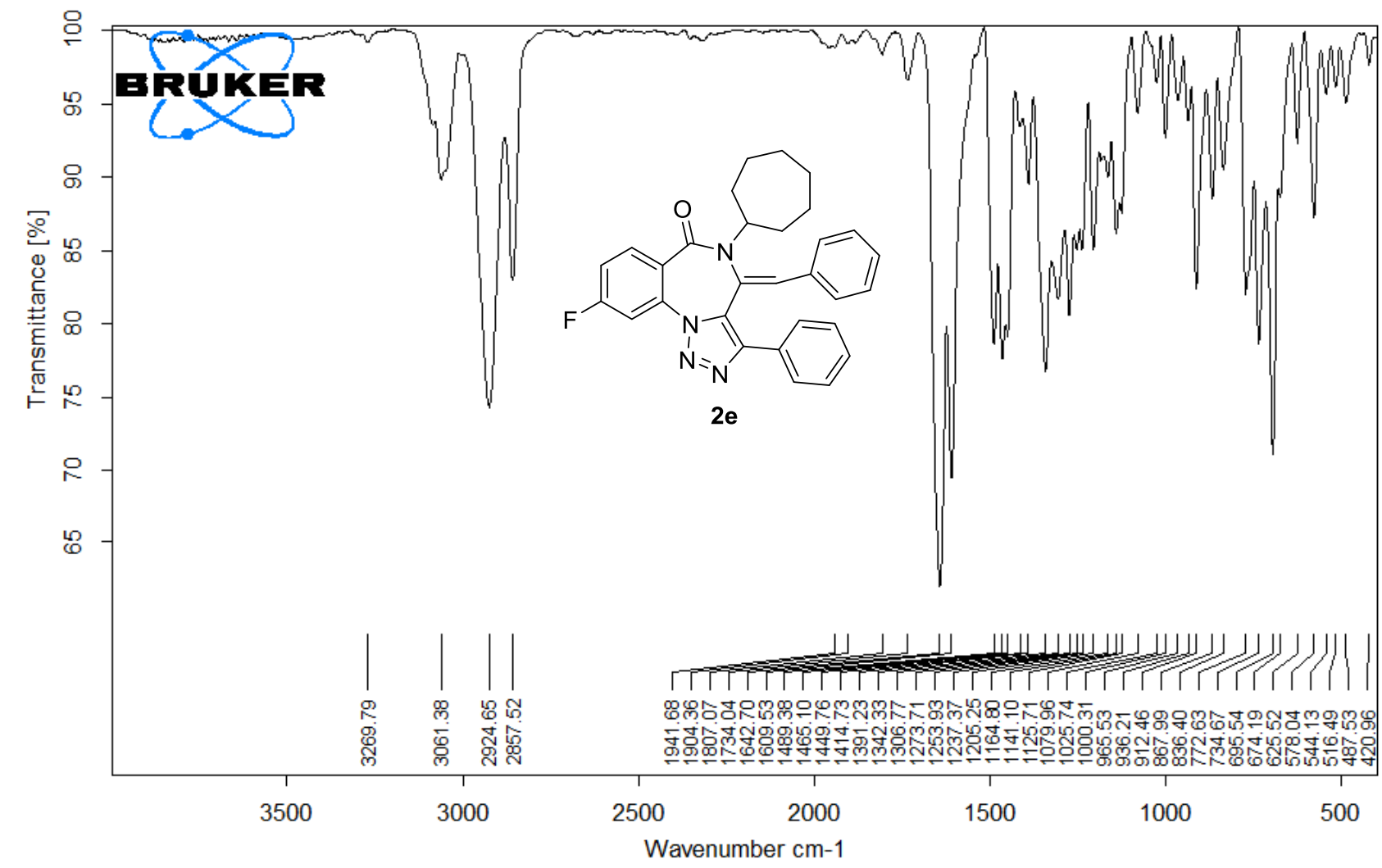

\begin{tabular}{|lcr|l|l|}
\hline D:IFTIR FILESI201808120180821IMIR_TR_DTGS_26117.0 $\quad$ MIR_TR_DTGS_26117 $\quad$ Instrument type and / or accessory & $8 / 21 / 2018$ \\
\hline
\end{tabular}

Page 1/1

IR of compound $\mathbf{2 e}$ 


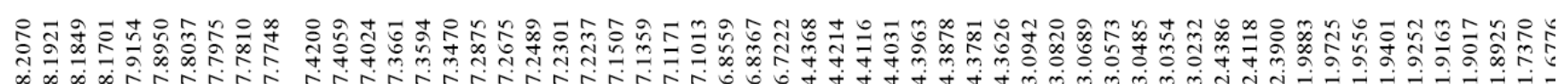

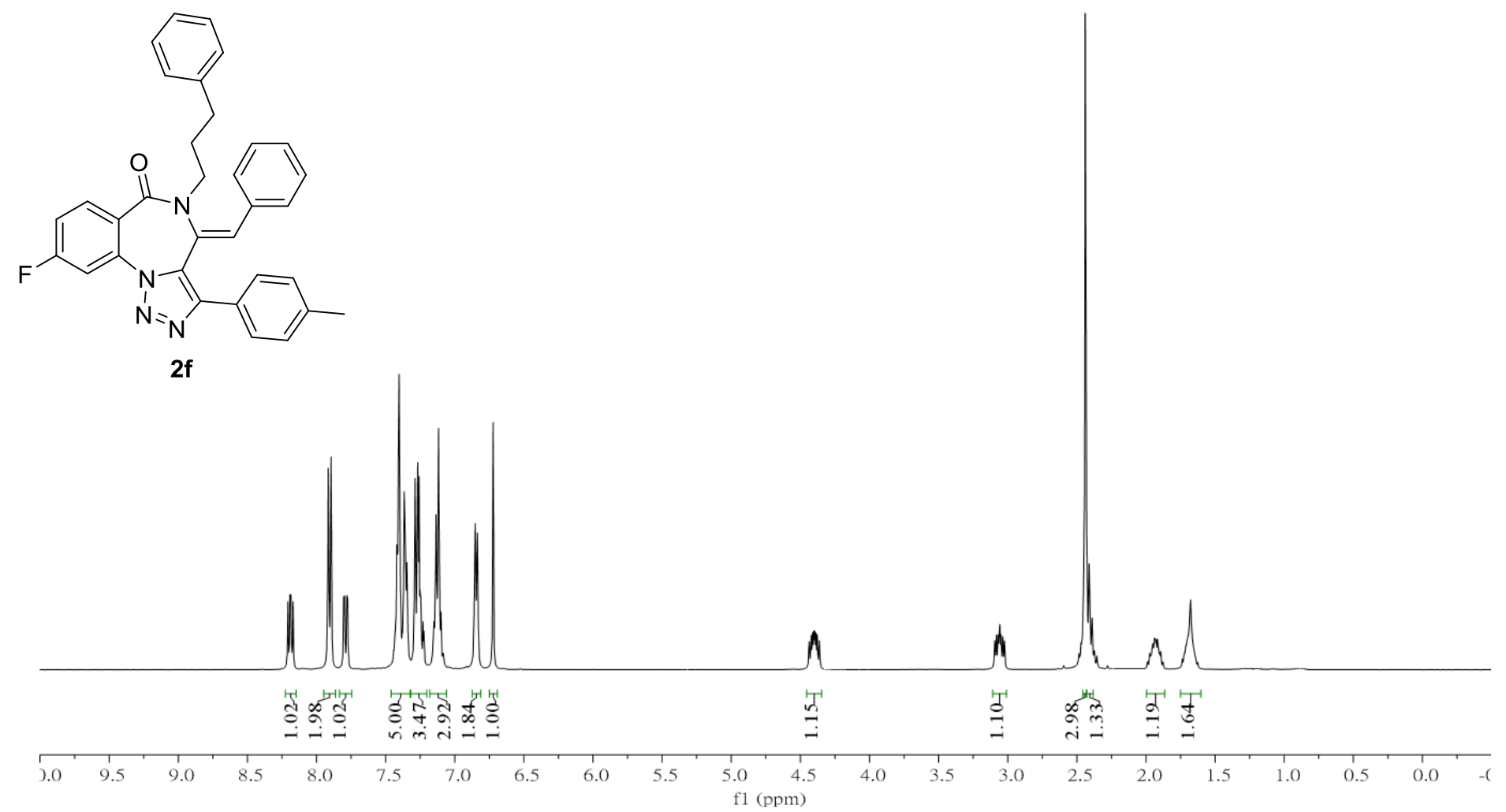

${ }^{1} \mathrm{H}$ NMR Spectrum (400 MHz) of compound $2 \mathbf{f}$ in $\mathrm{CDCl}_{3}$ 

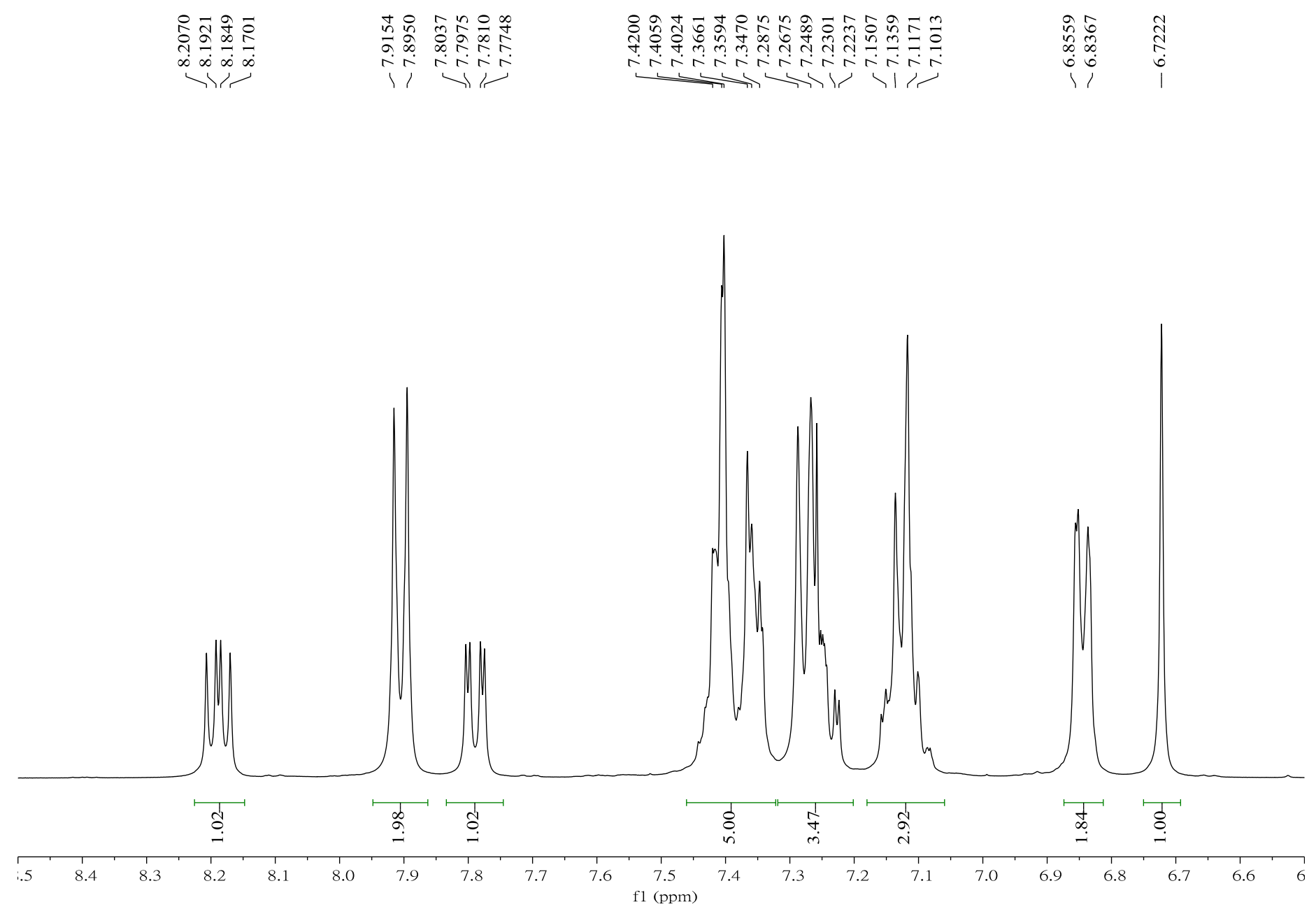

Expansion of ${ }^{1} \mathrm{H}$ NMR Spectrum $(400 \mathrm{MHz})$ of compound $2 \mathbf{f}$ in $\mathrm{CDCl}_{3}$ 


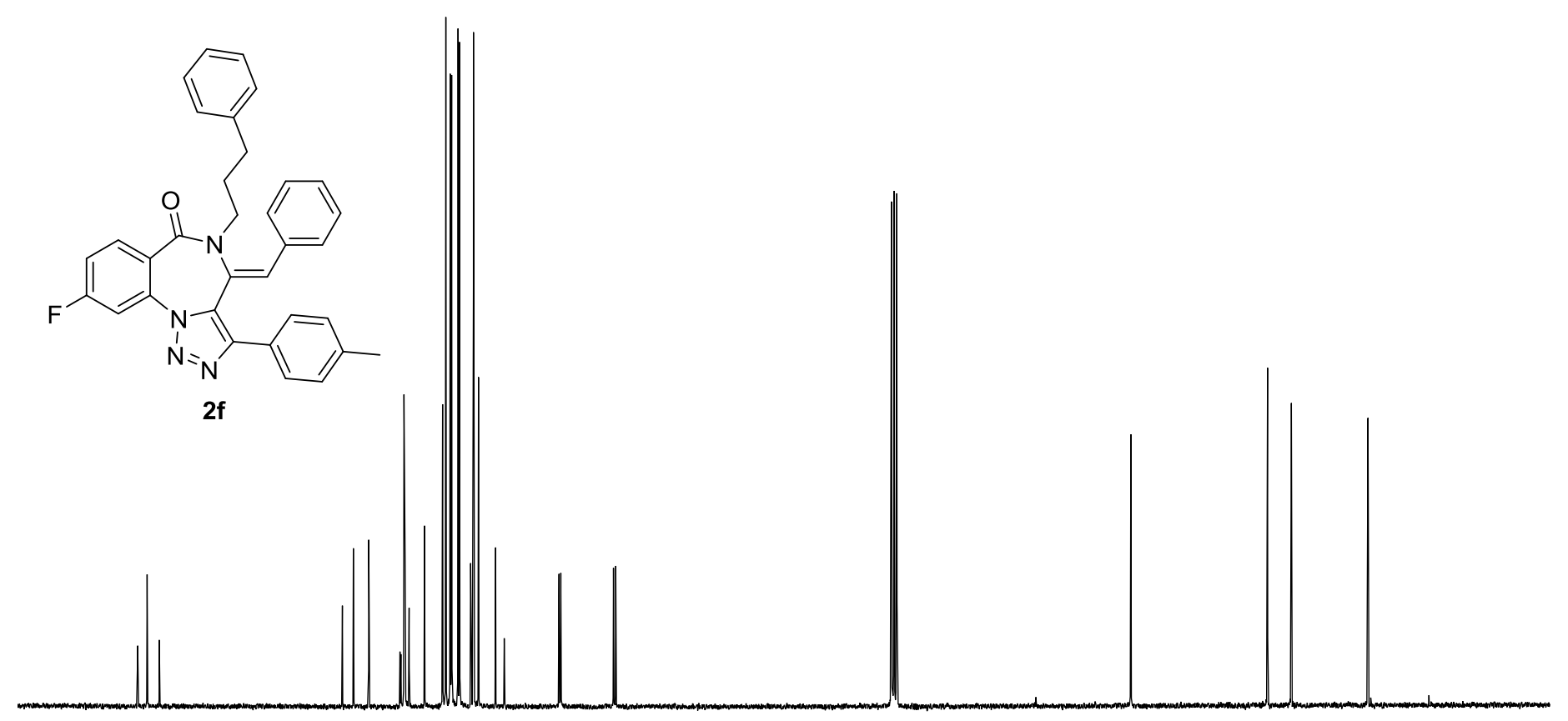

\begin{tabular}{lllll}
\hline 80 & 170 & 160 & 150 & 140
\end{tabular} $130 \quad 120$ 110

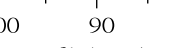

$80 \quad 70$

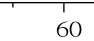

50 40

${ }^{13} \mathrm{C}$ NMR Spectrum (101 MHz) of compound $2 \mathbf{f}$ in $\mathrm{CDCl}_{3}$ 


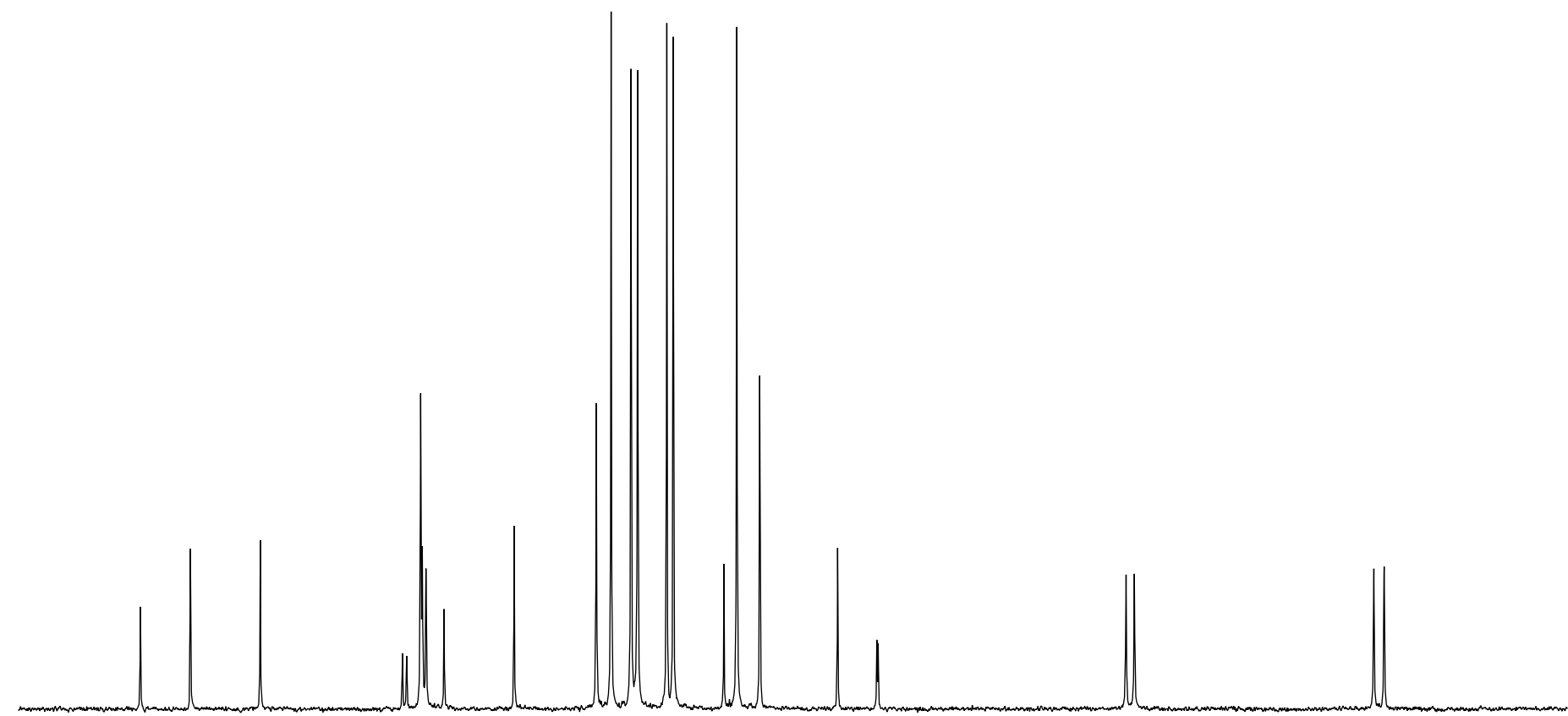

451441431421411401391381371361351341331321311301291281271261251241231221211201191181171161151141131121111101091081071061 f1 (ppm)

Expansion of ${ }^{13} \mathrm{C}$ NMR Spectrum $(101 \mathrm{MHz})$ of compound $2 \mathbf{f}$ in $\mathrm{CDCl}_{3}$ 


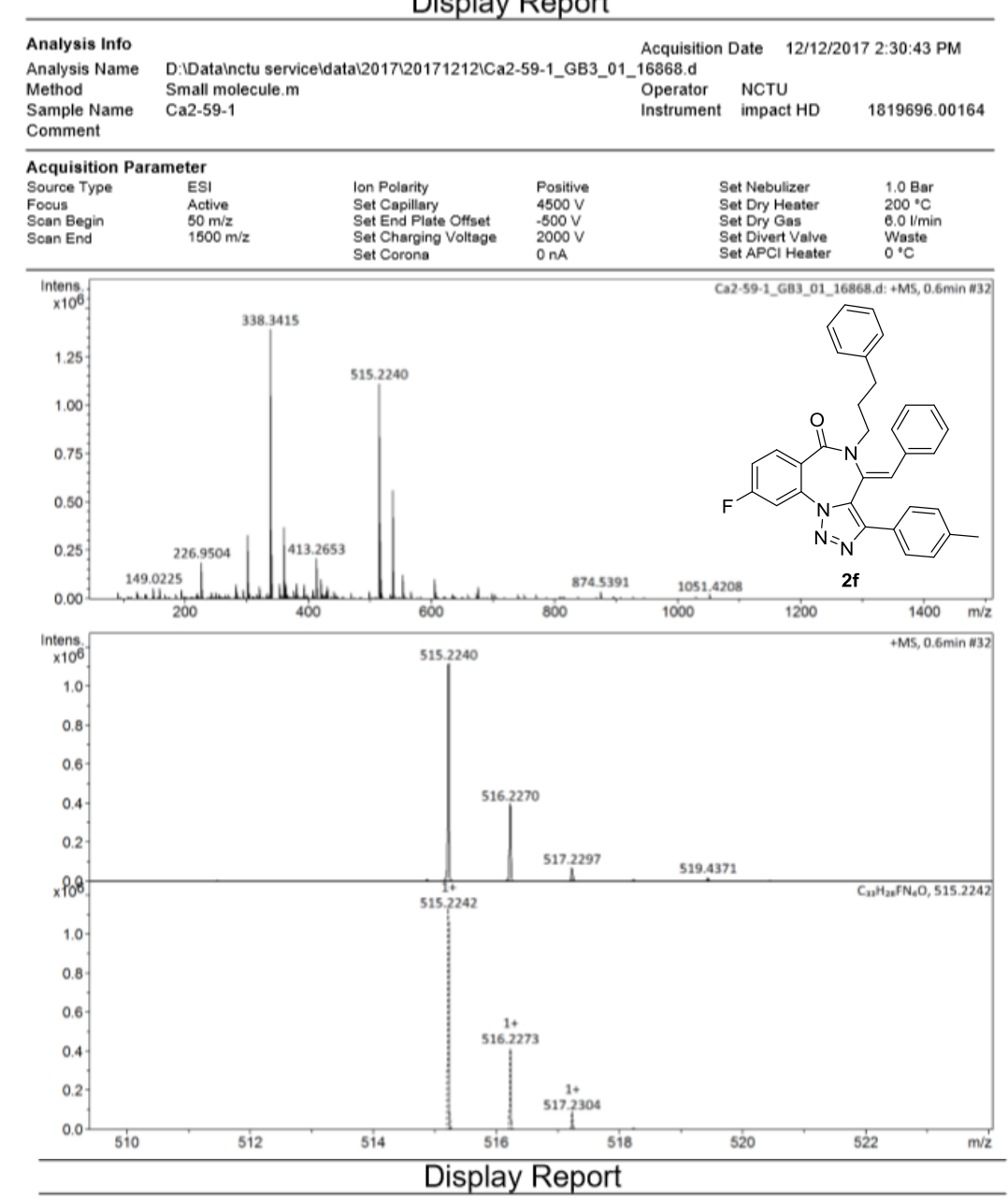

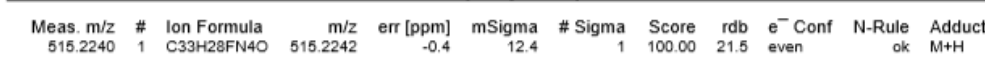

HRMS of compound $2 \mathbf{f}$ 


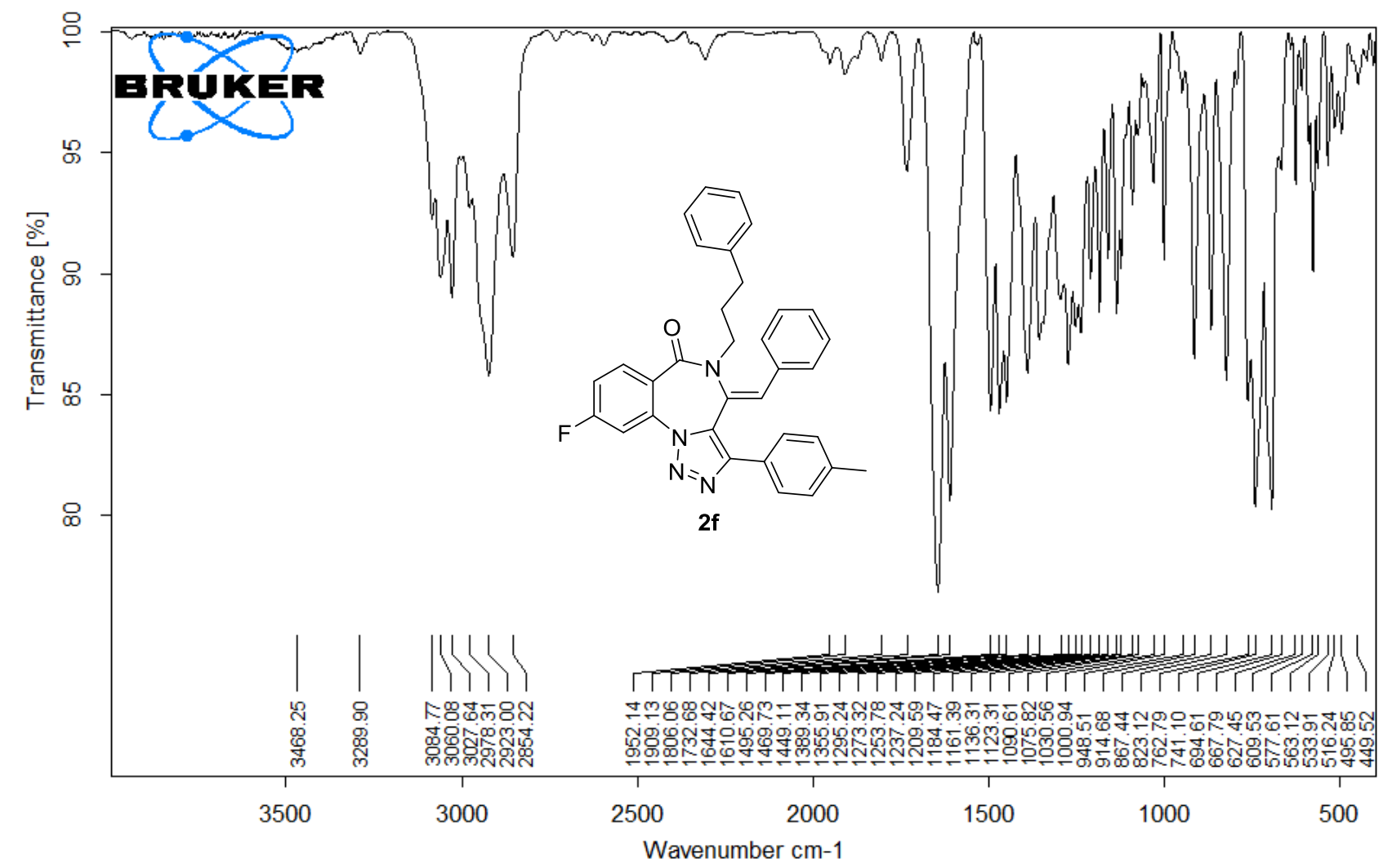

\begin{tabular}{|lcr|l|}
\hline D:IFTIR FILESI201808120180821MIR_TR_DTGS_26118.0 $\quad$ MIR_TR_DTGS_26118 $\quad$ Instrument type and / or accessory & $8 / 21 / 2018$ \\
\hline
\end{tabular}

Page 1/1

IR of compound $\mathbf{2 f}$ 


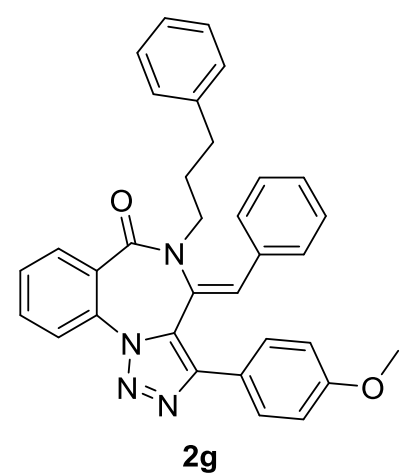

$2 \mathrm{~g}$

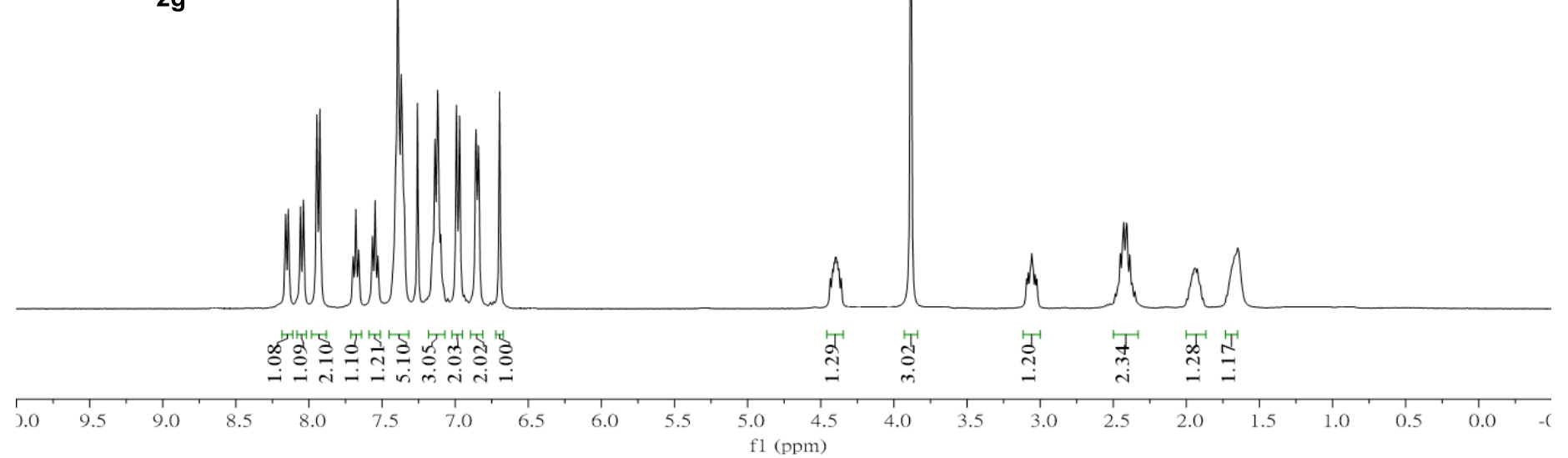

${ }^{1} \mathrm{H}$ NMR Spectrum (400 MHz) of compound $\mathbf{2 g}$ in $\mathrm{CDCl}_{3}$ 


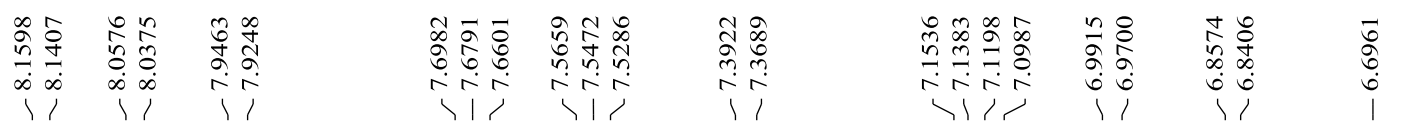

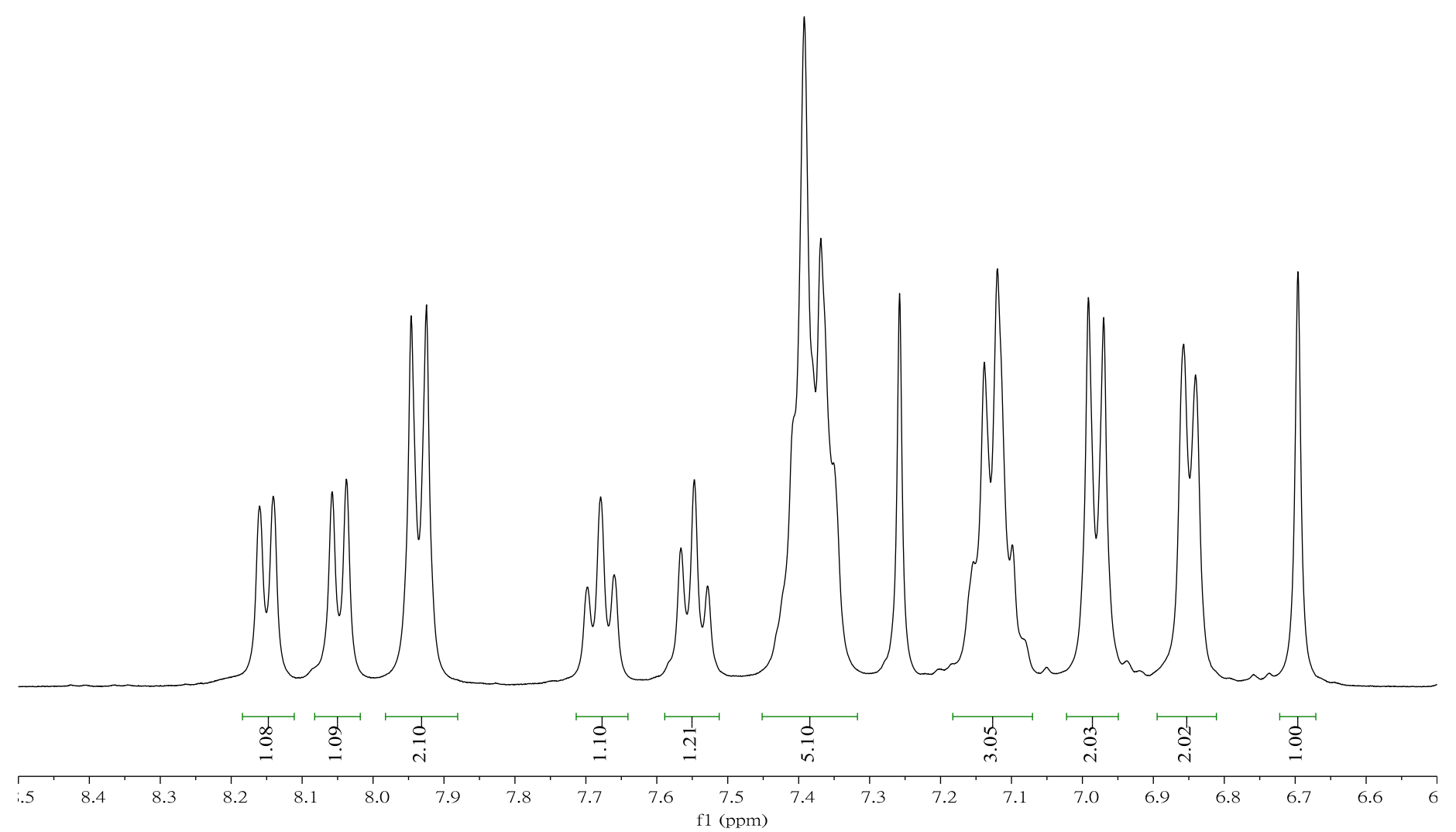

Expansion of ${ }^{1} \mathrm{H}$ NMR Spectrum (400 MHz) of compound $\mathbf{2 g}$ in $\mathrm{CDCl}_{3}$ 


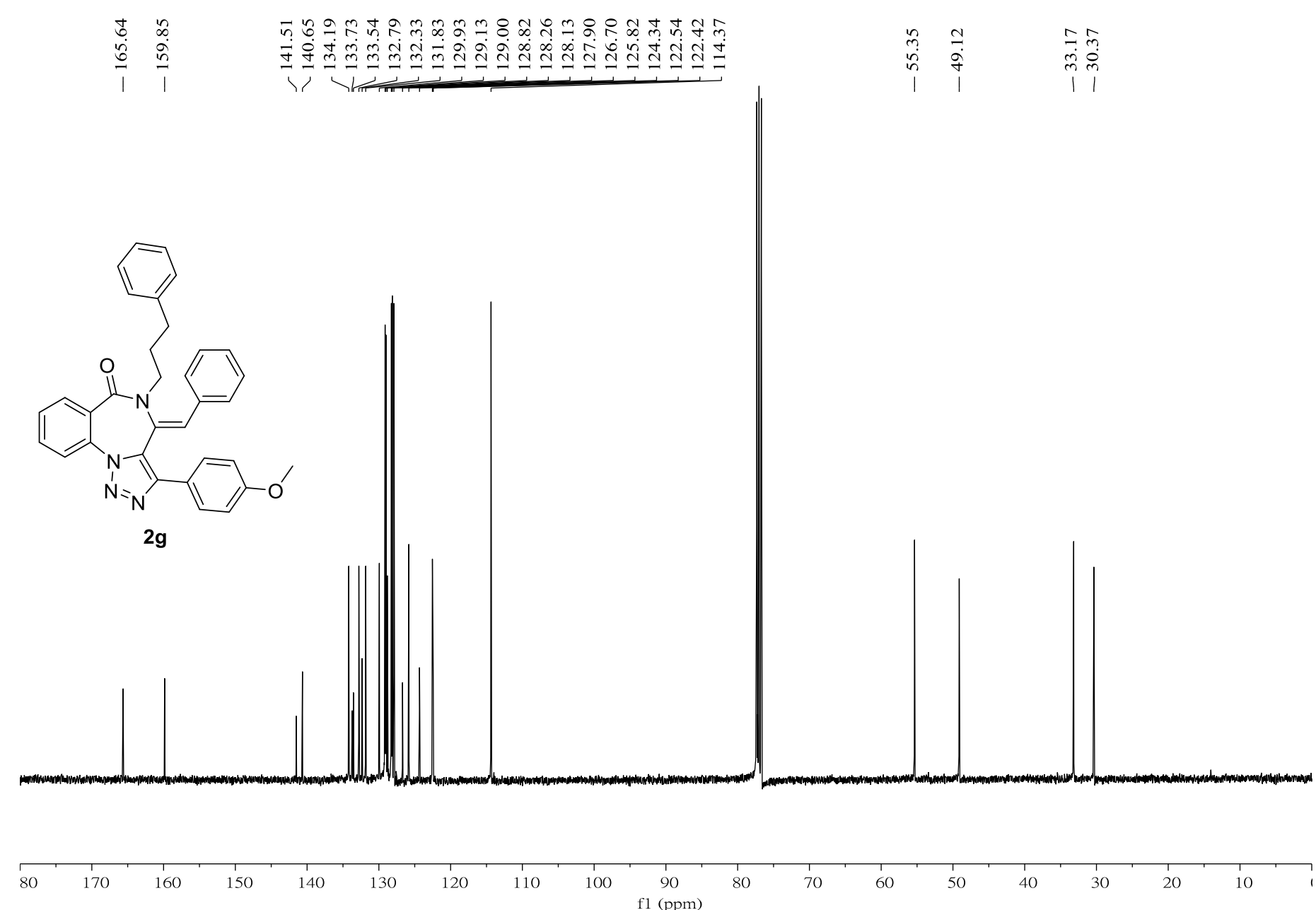

${ }^{13} \mathrm{C}$ NMR Spectrum (101 MHz) of compound $2 \mathrm{~g}$ in $\mathrm{CDCl}_{3}$ 


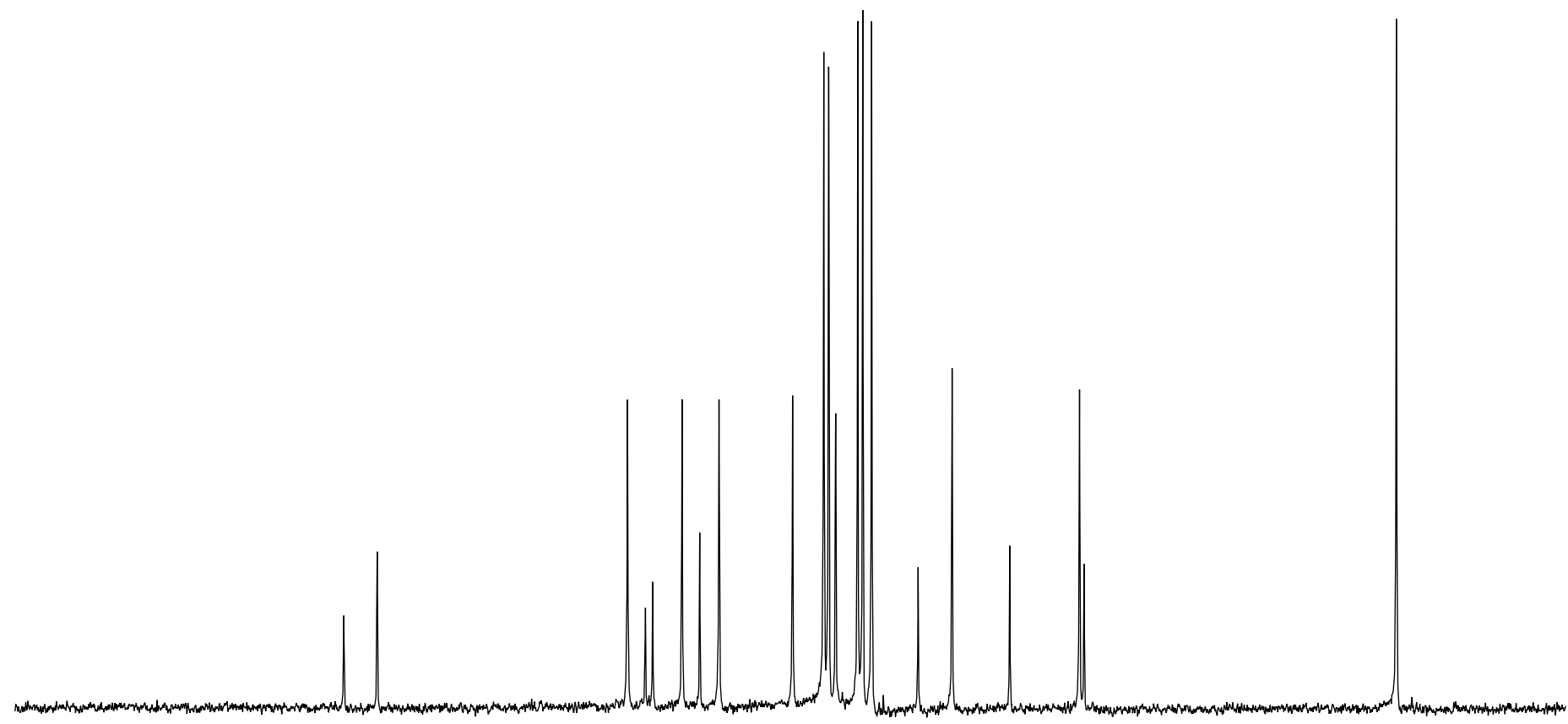

50149148147146145144143142141140139138137136135134133132131130129128127126125124123122121120119118117116115114113112111 $\mathrm{f} 1$ (ppm)

Expansion of ${ }^{13} \mathrm{C}$ NMR Spectrum (101 MHz) of compound $2 \mathrm{~g}$ in $\mathrm{CDCl}_{3}$ 
Display Report

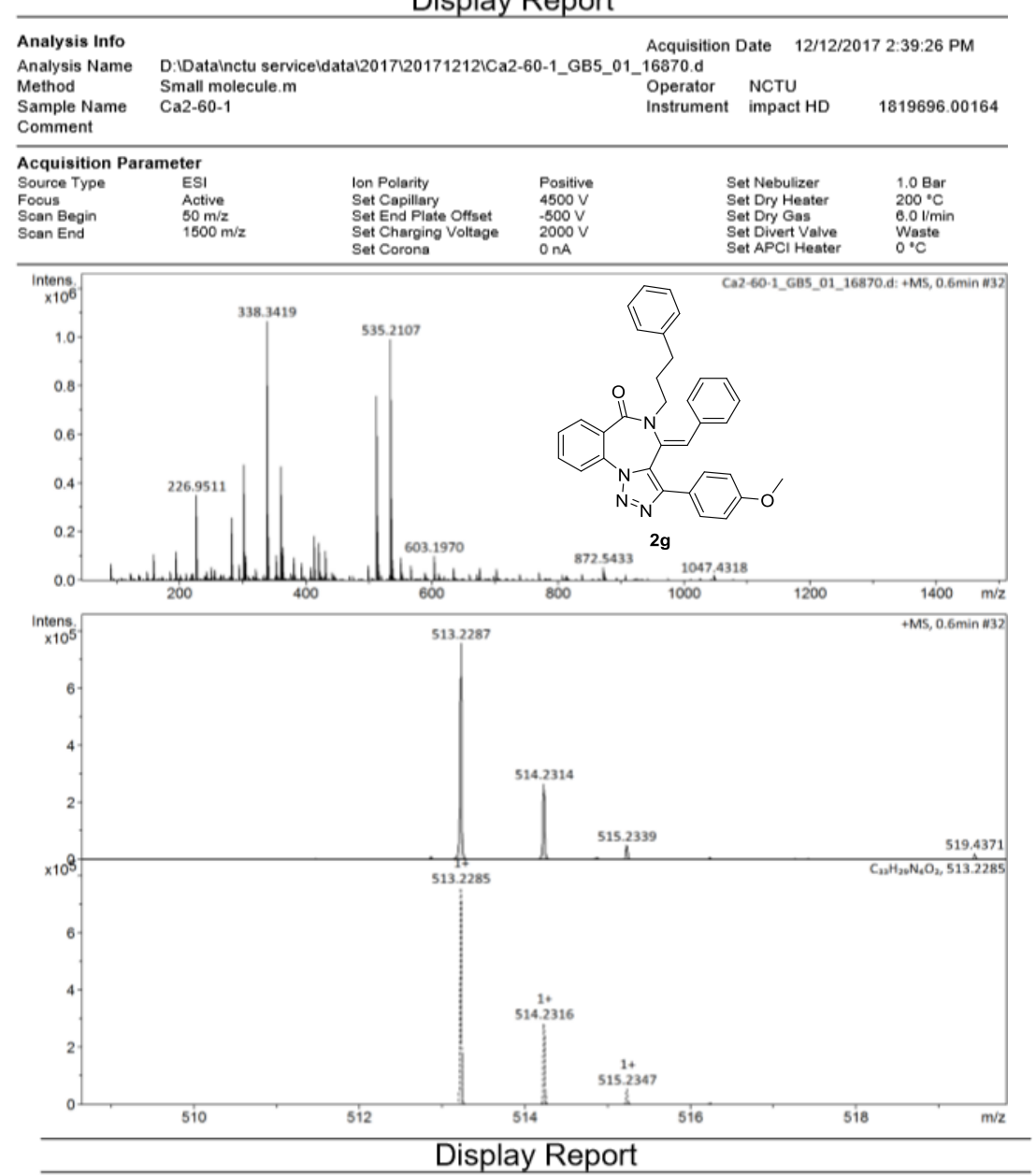

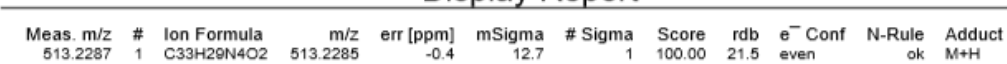

HRMS of compound $\mathbf{2 g}$ 


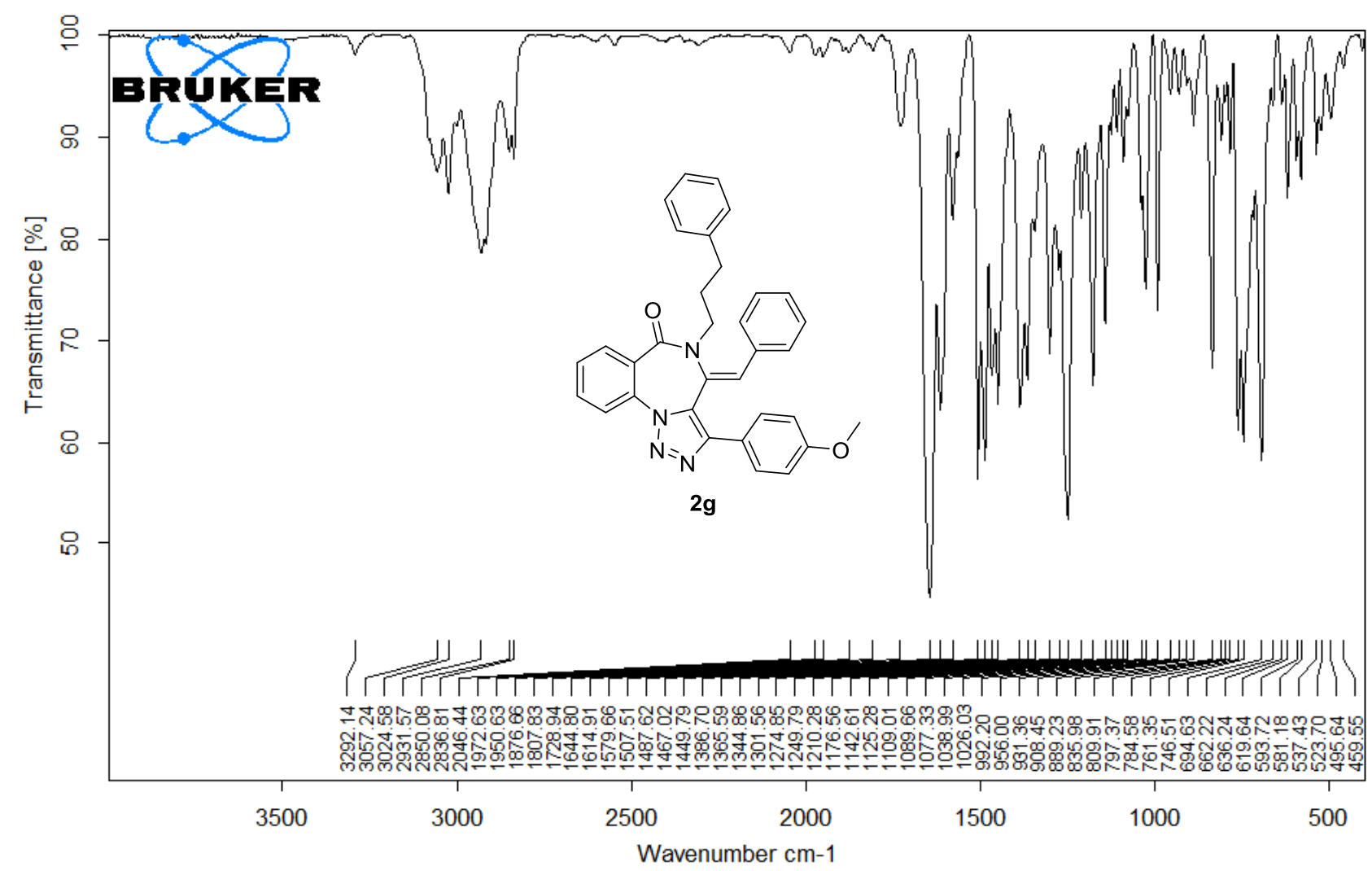

\begin{tabular}{|lcl|l|}
\hline D:IFTIR FILESI201808120180821IMIR_TR_DTGS_26119.0 $\quad$ MIR_TR_DTGS_26119 $\quad$ Instrument type and / or accessory & $8 / 21 / 2018$ \\
\hline
\end{tabular}

Page 1/1

IR of compound $\mathbf{2 g}$ 
L

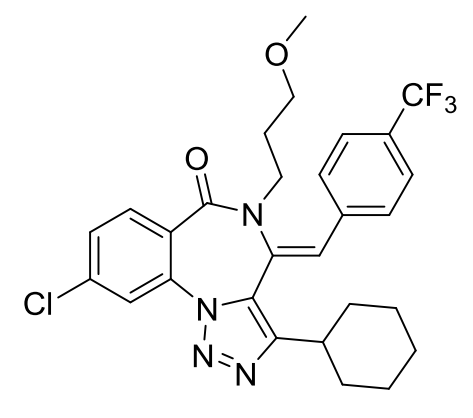

2h

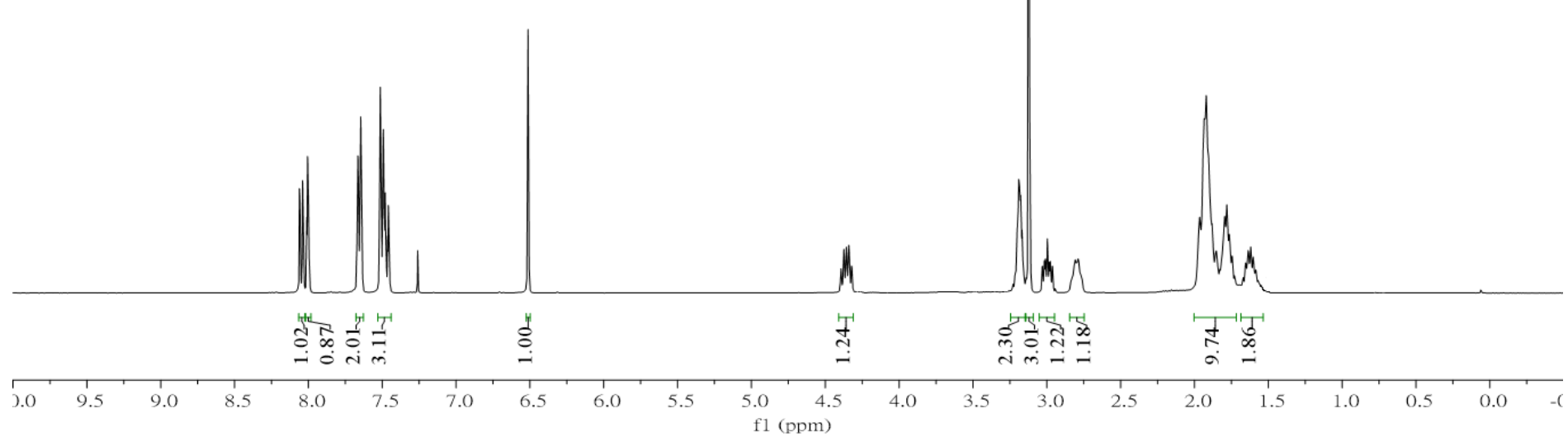

${ }^{1} \mathrm{H}$ NMR Spectrum (400 MHz) of compound $\mathbf{2 h}$ in $\mathrm{CDCl}_{3}$ 


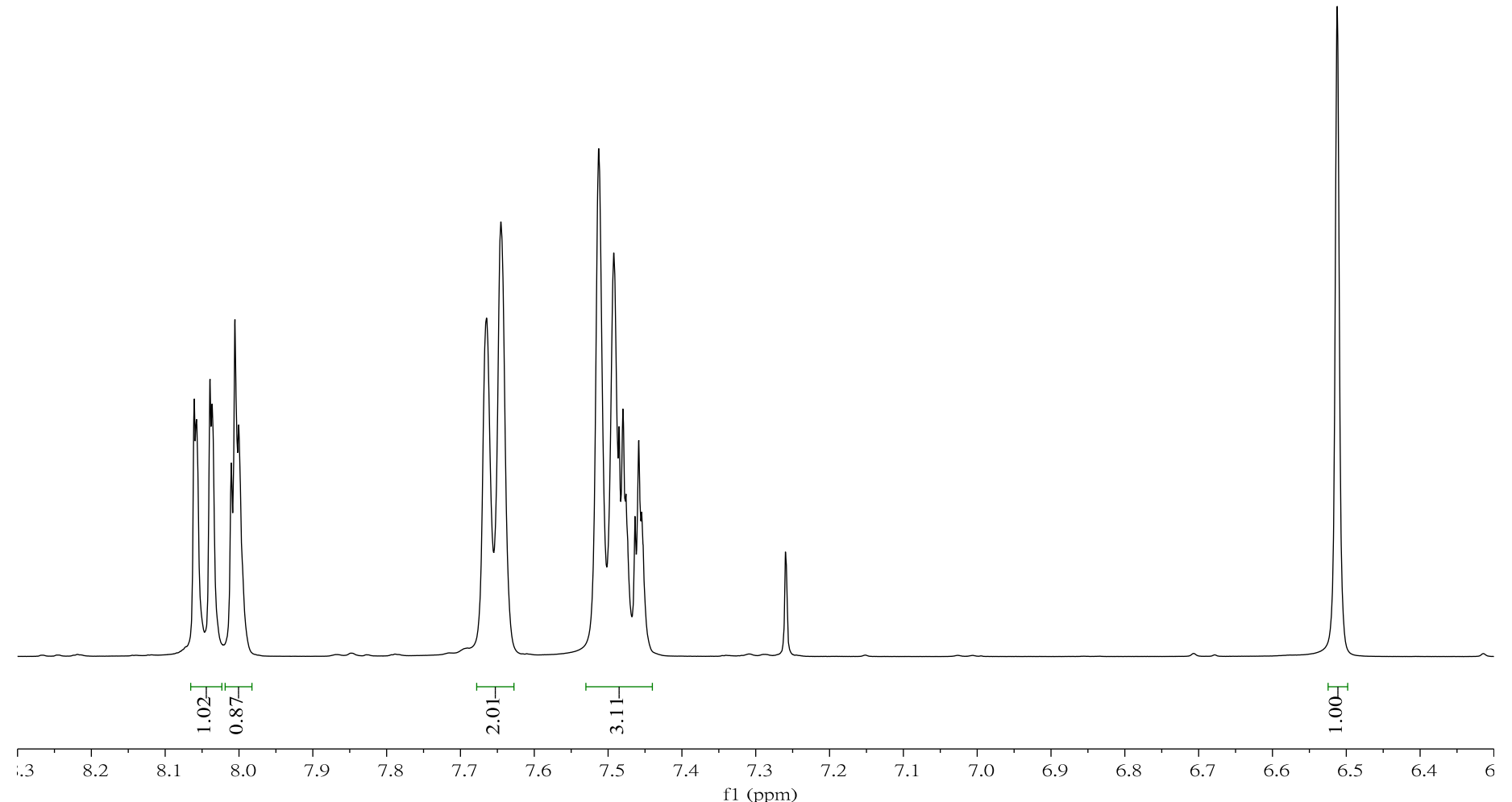

Expansion of ${ }^{1} \mathrm{H}$ NMR Spectrum (400 MHz) of compound $\mathbf{2 h}$ in $\mathrm{CDCl}_{3}$ 


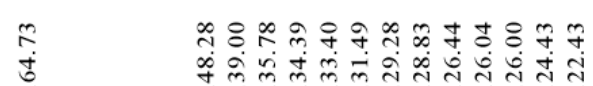

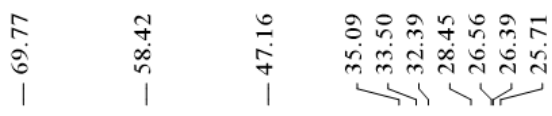

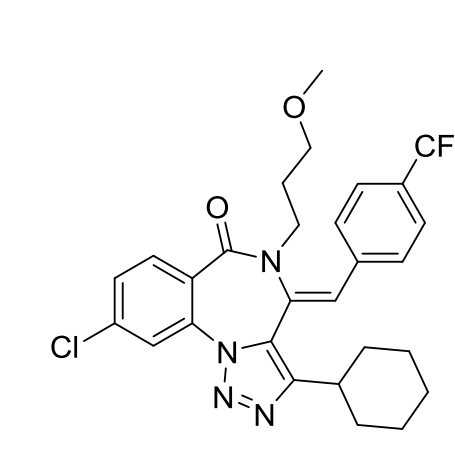

$2 h$

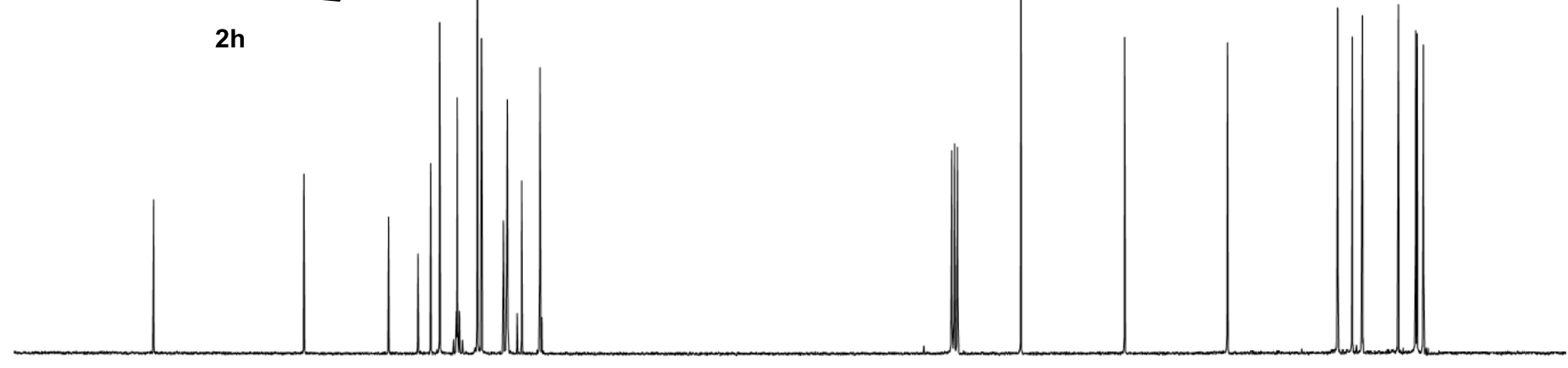

$80 \quad 170 \quad 160 \quad 150 \quad 140$
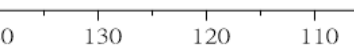

$100 \quad 90$

80

70

60

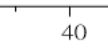

30 201

${ }^{13} \mathrm{C}$ NMR Spectrum (101 MHz) of compound $\mathbf{2 h}$ in $\mathrm{CDCl}_{3}$ 


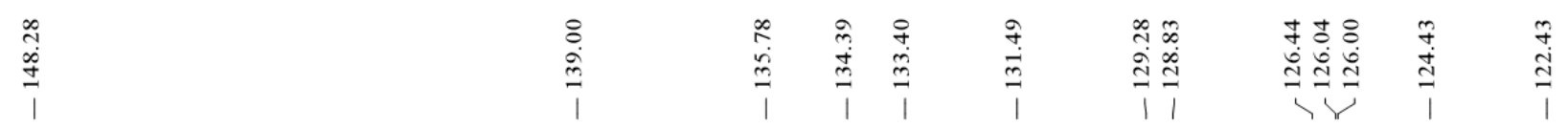

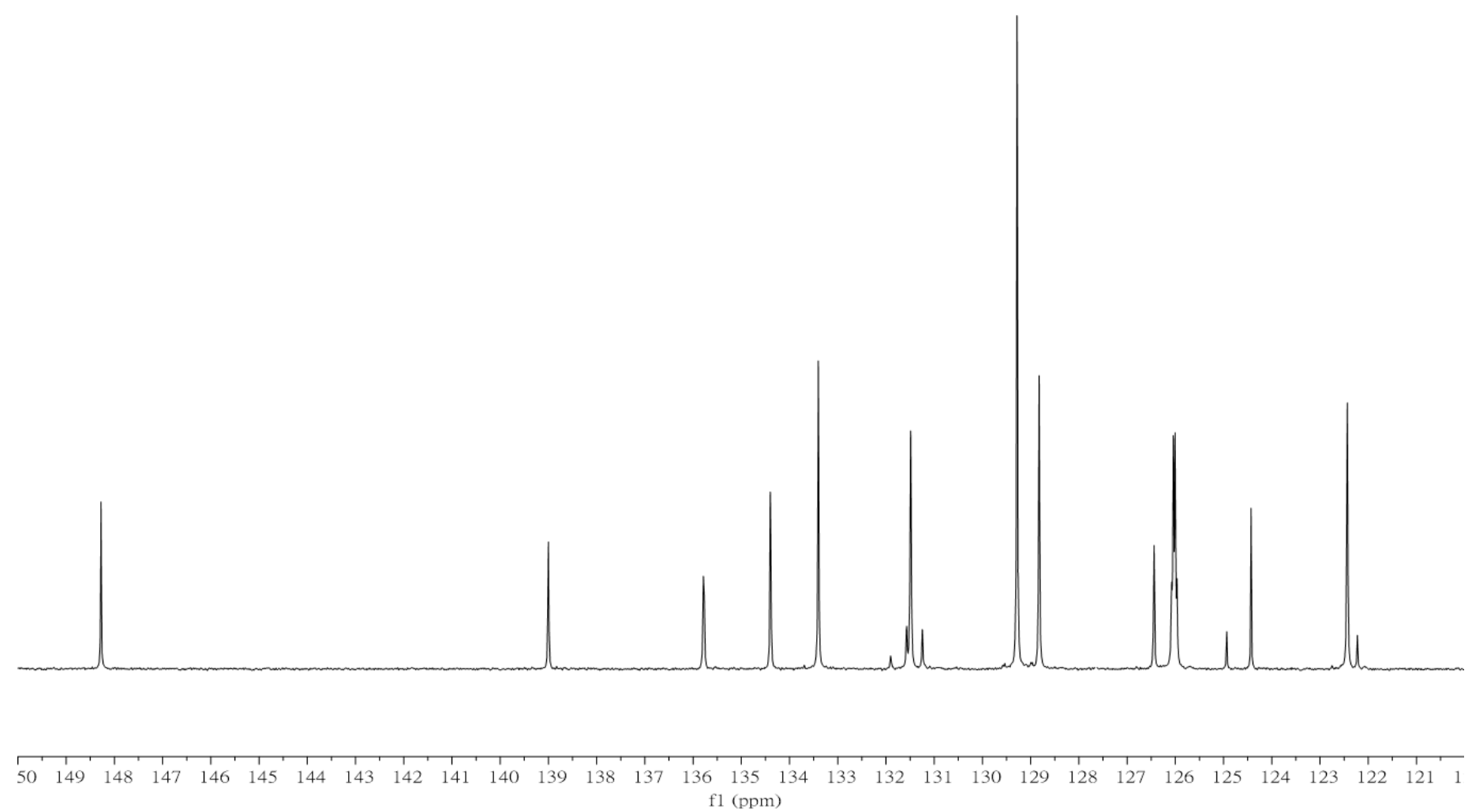

Expansion of ${ }^{13} \mathrm{C}$ NMR Spectrum $(101 \mathrm{MHz})$ of compound $\mathbf{2 h}$ in $\mathrm{CDCl}_{3}$ 


\section{Display Report}

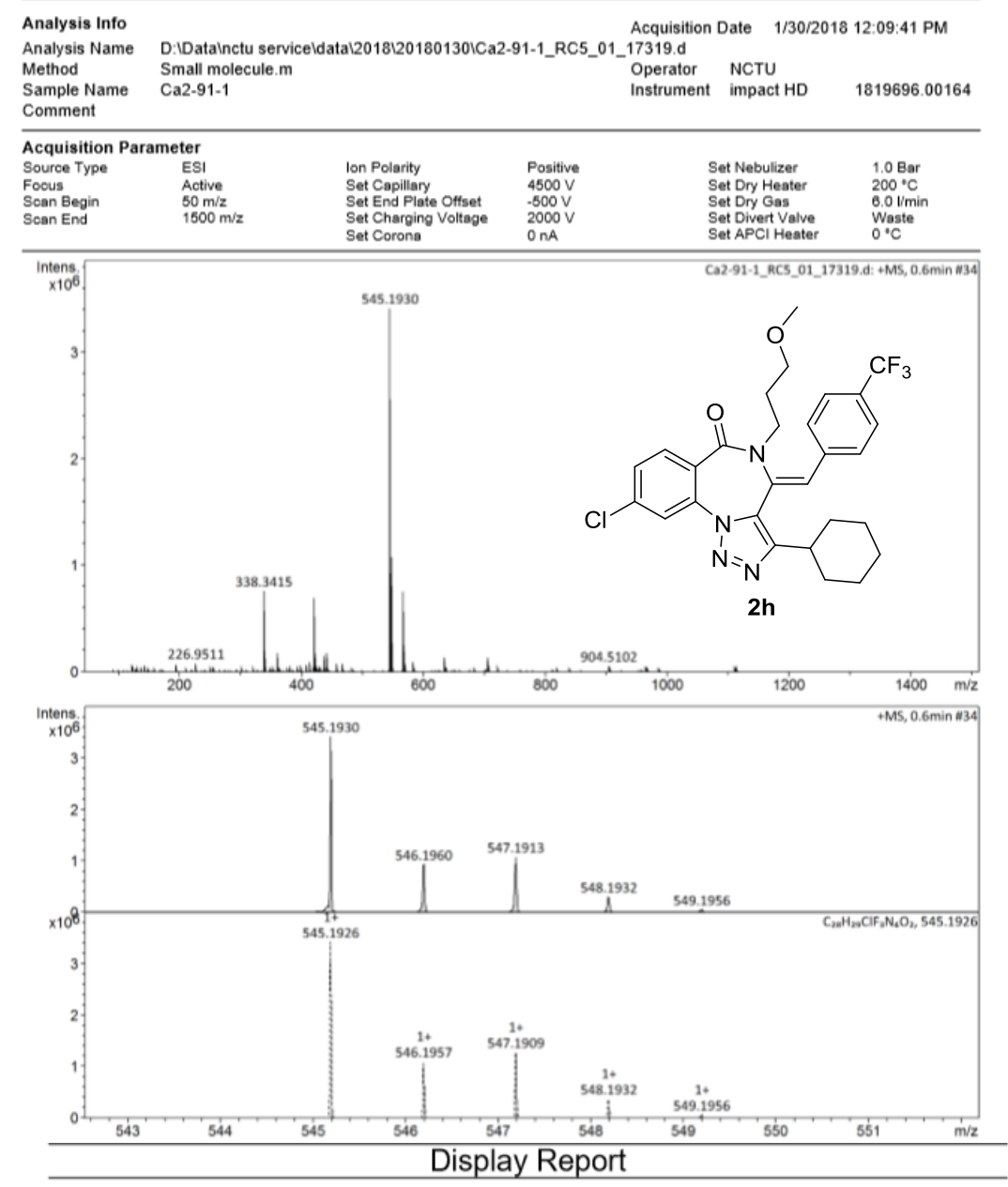

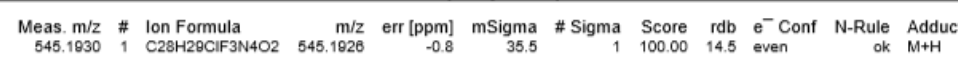

HRMS of compound $\mathbf{2 h}$ 


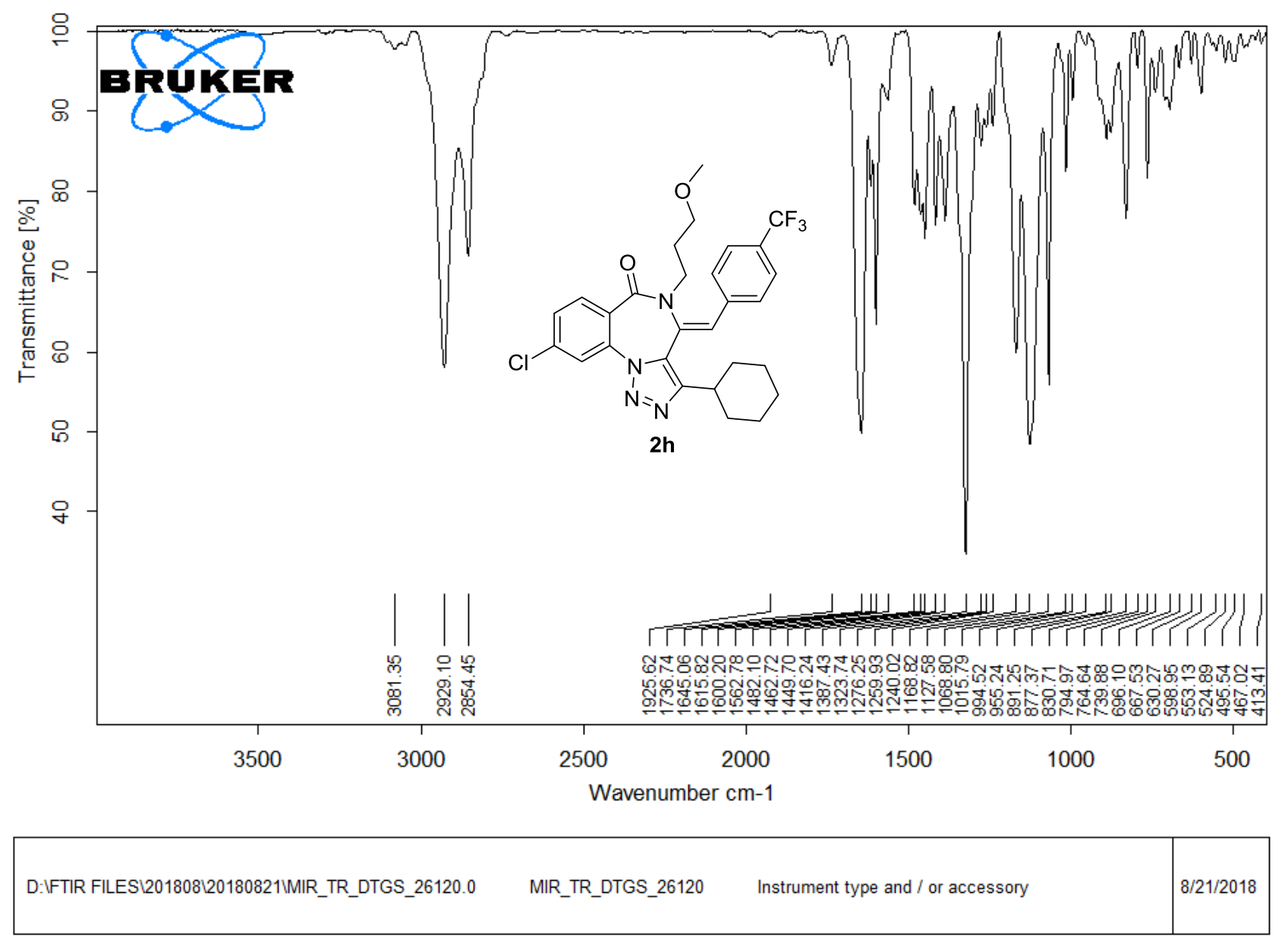

Page 1/1

IR of compound $\mathbf{2 h}$ 


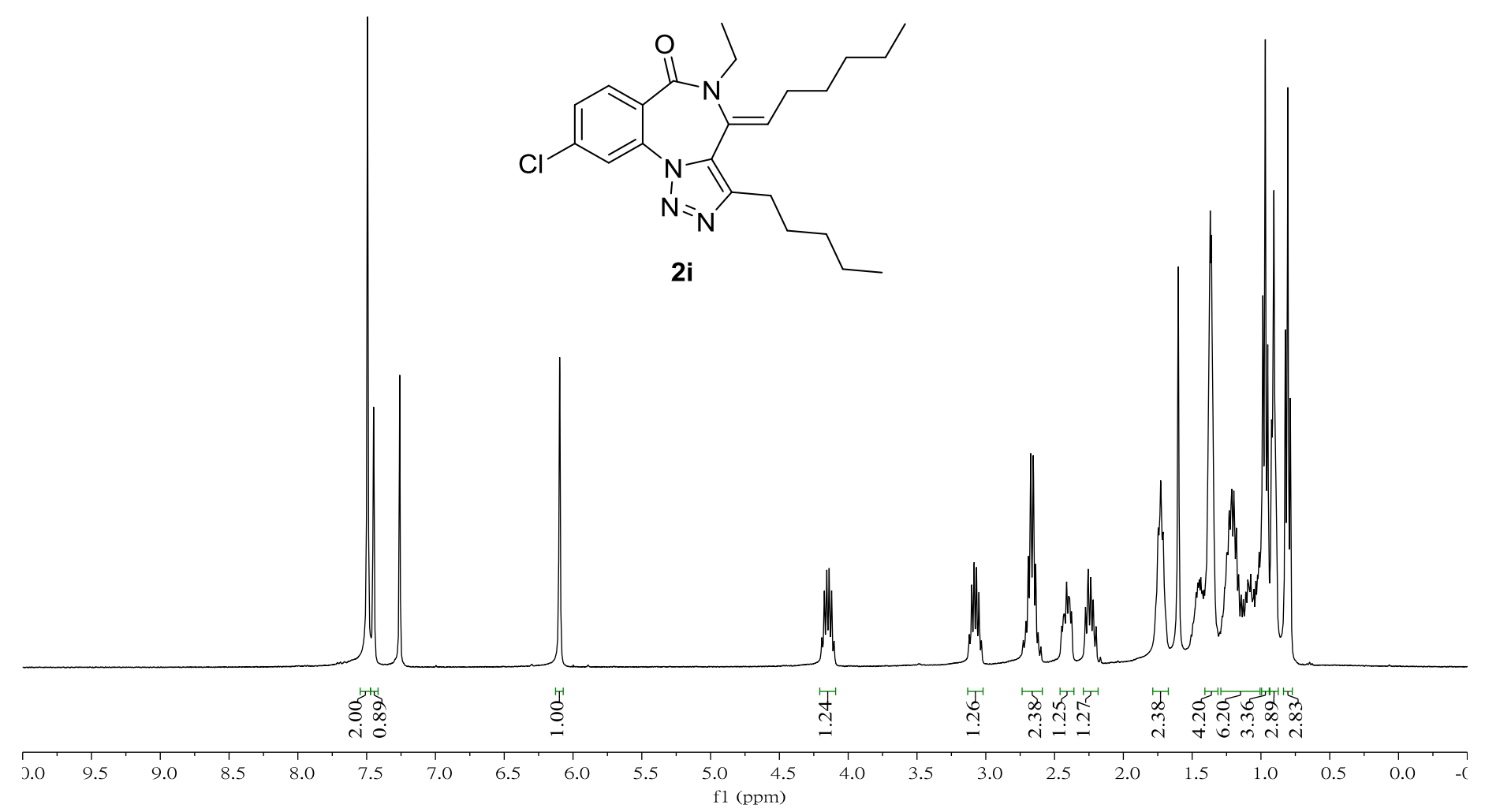

${ }^{1} \mathrm{H}$ NMR Spectrum (400 MHz) of compound $2 \mathbf{i}$ in $\mathrm{CDCl}_{3}$ 


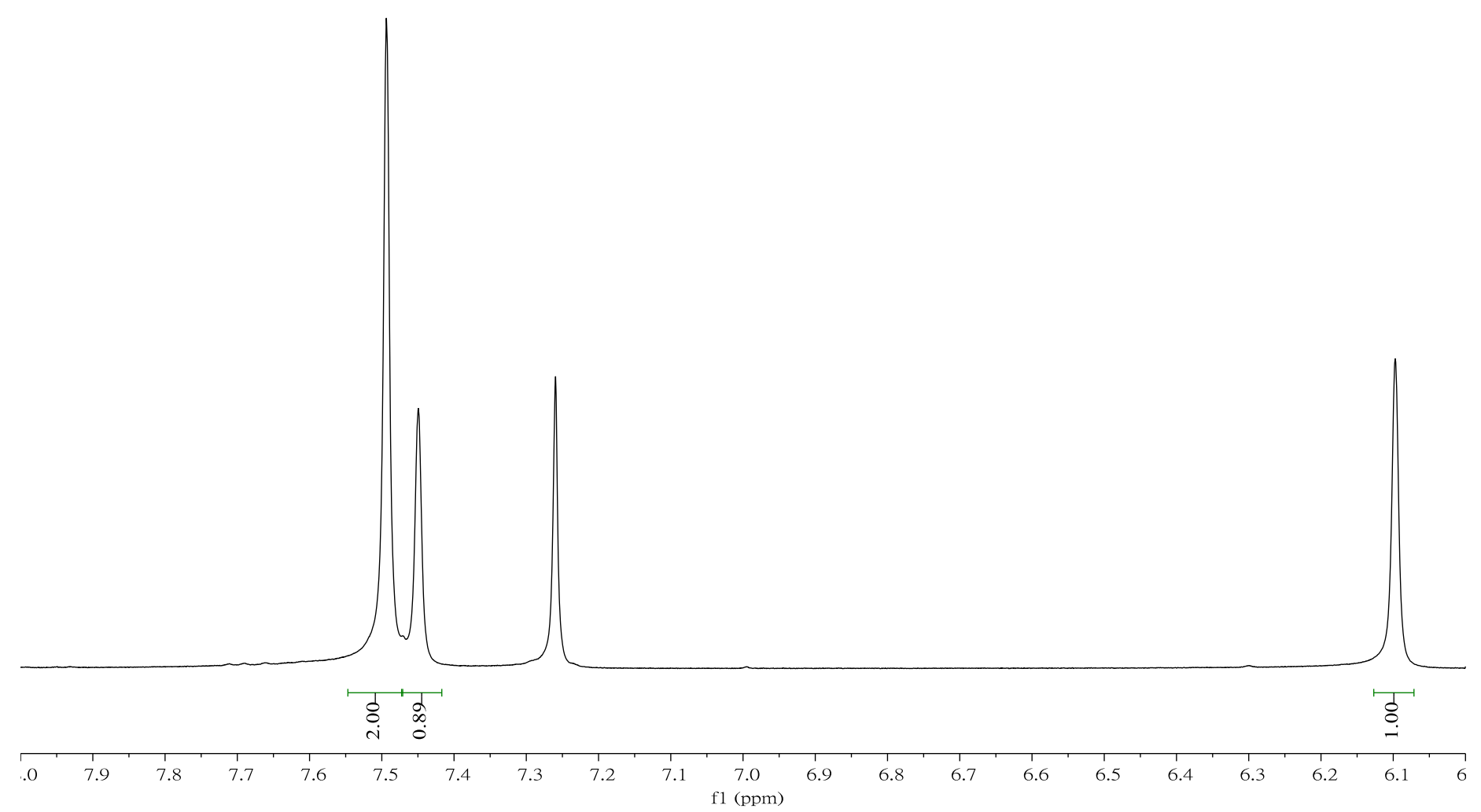

Expansion of ${ }^{1} \mathrm{H}$ NMR Spectrum (400 MHz) of compound $\mathbf{2 i}$ in $\mathrm{CDCl}_{3}$ 


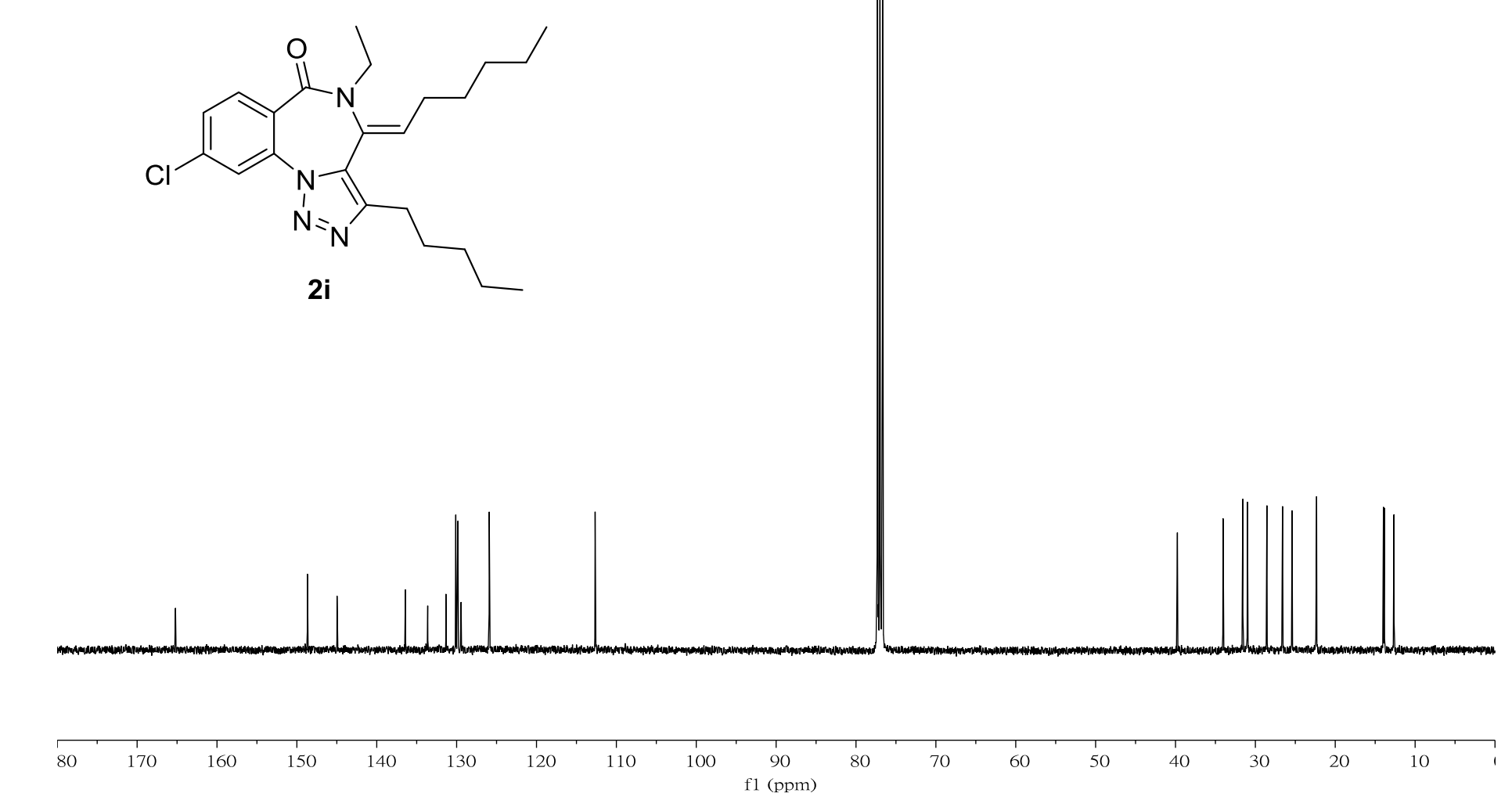

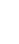

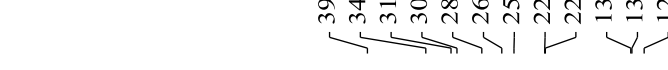

${ }^{13} \mathrm{C}$ NMR Spectrum $(101 \mathrm{MHz})$ of compound $\mathbf{2} \mathbf{i}$ in $\mathrm{CDCl}_{3}$ 


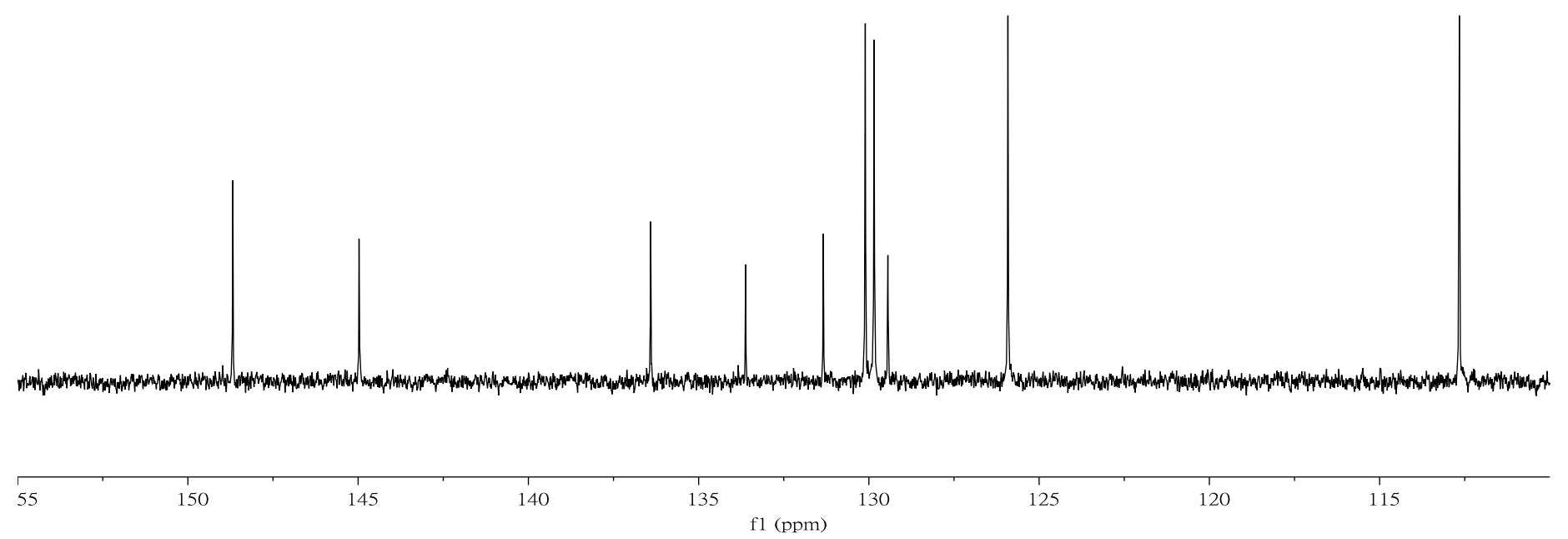

Expansion of ${ }^{13} \mathrm{C}$ NMR Spectrum $(101 \mathrm{MHz})$ of compound $2 \mathbf{i}$ in $\mathrm{CDCl}_{3}$ 
Display Report

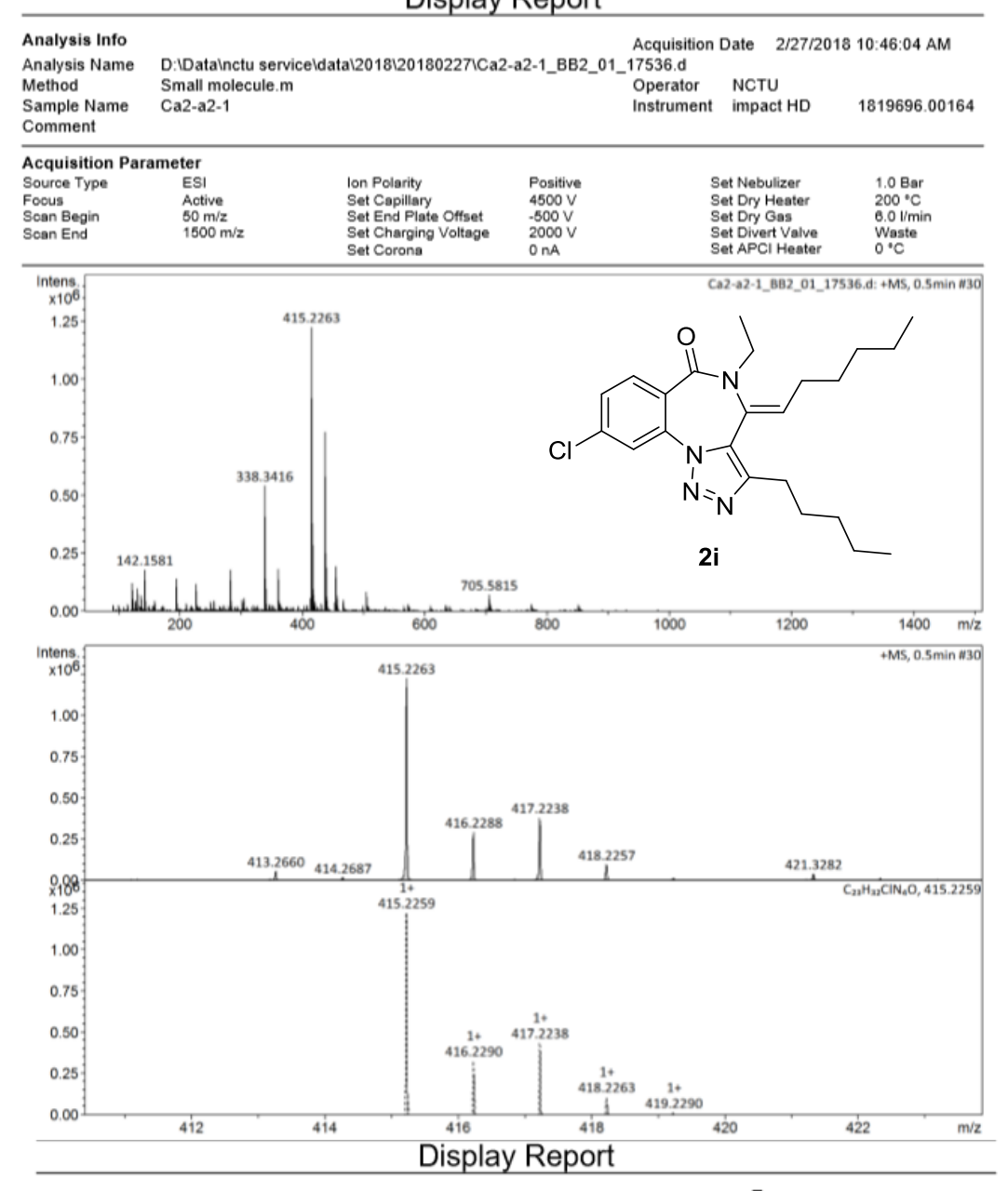

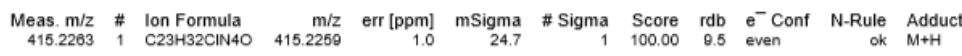

HRMS of compound $2 \mathbf{i}$ 


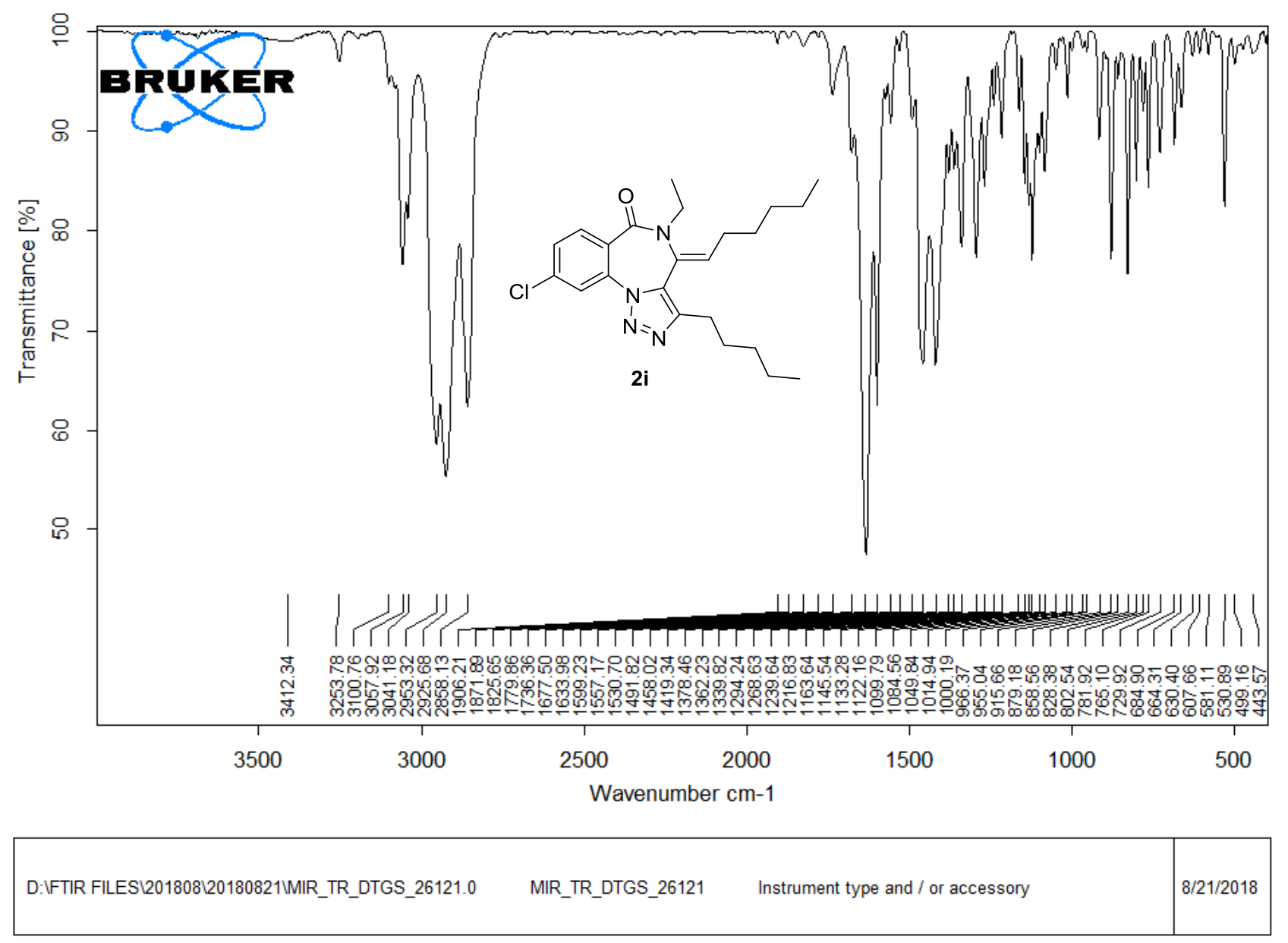

Page 1/1

IR of compound $2 \mathbf{i}$ 

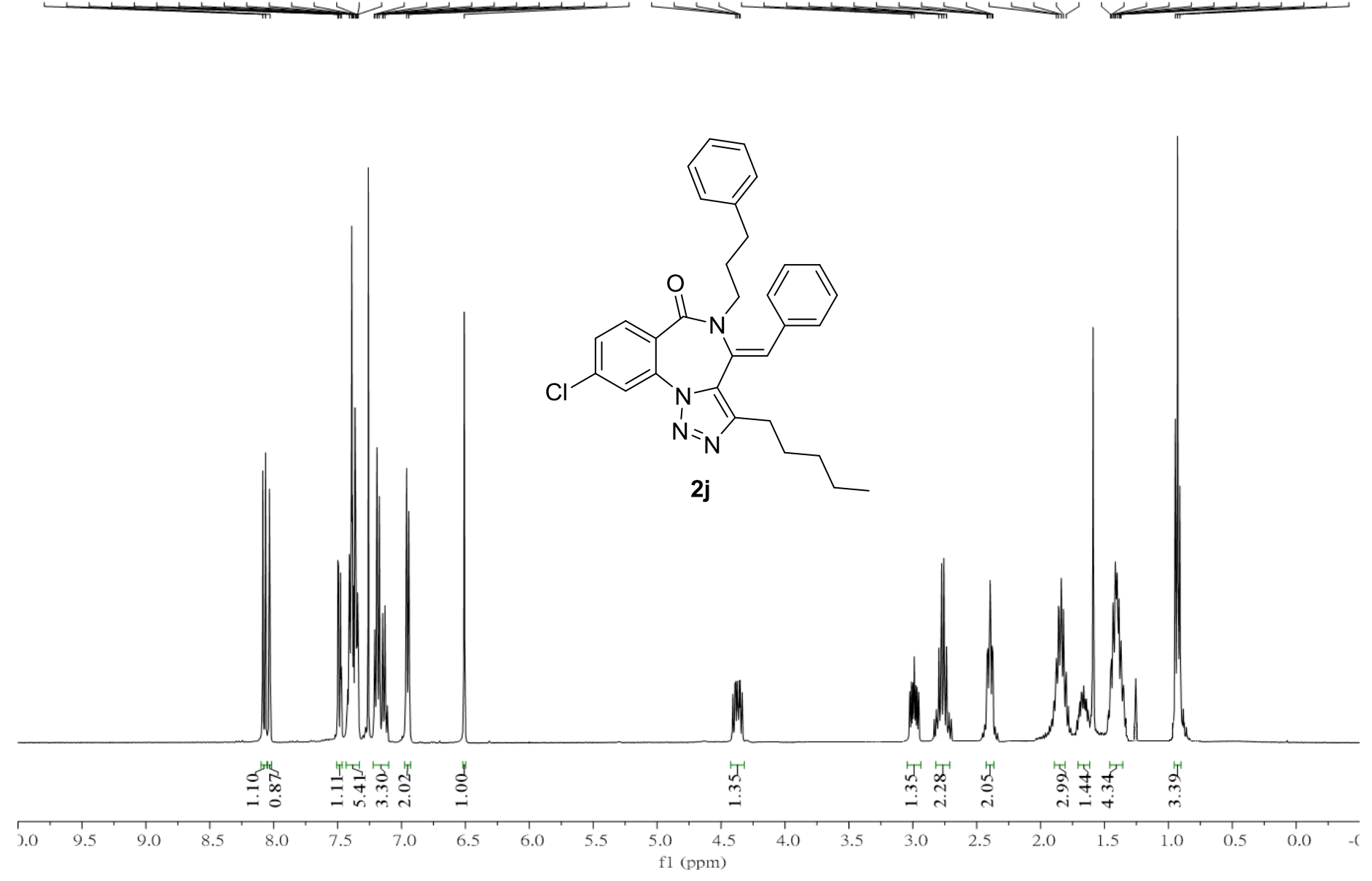

${ }^{1} \mathrm{H}$ NMR Spectrum (400 MHz) of compound $\mathbf{2} \mathbf{j}$ in $\mathrm{CDCl}_{3}$ 


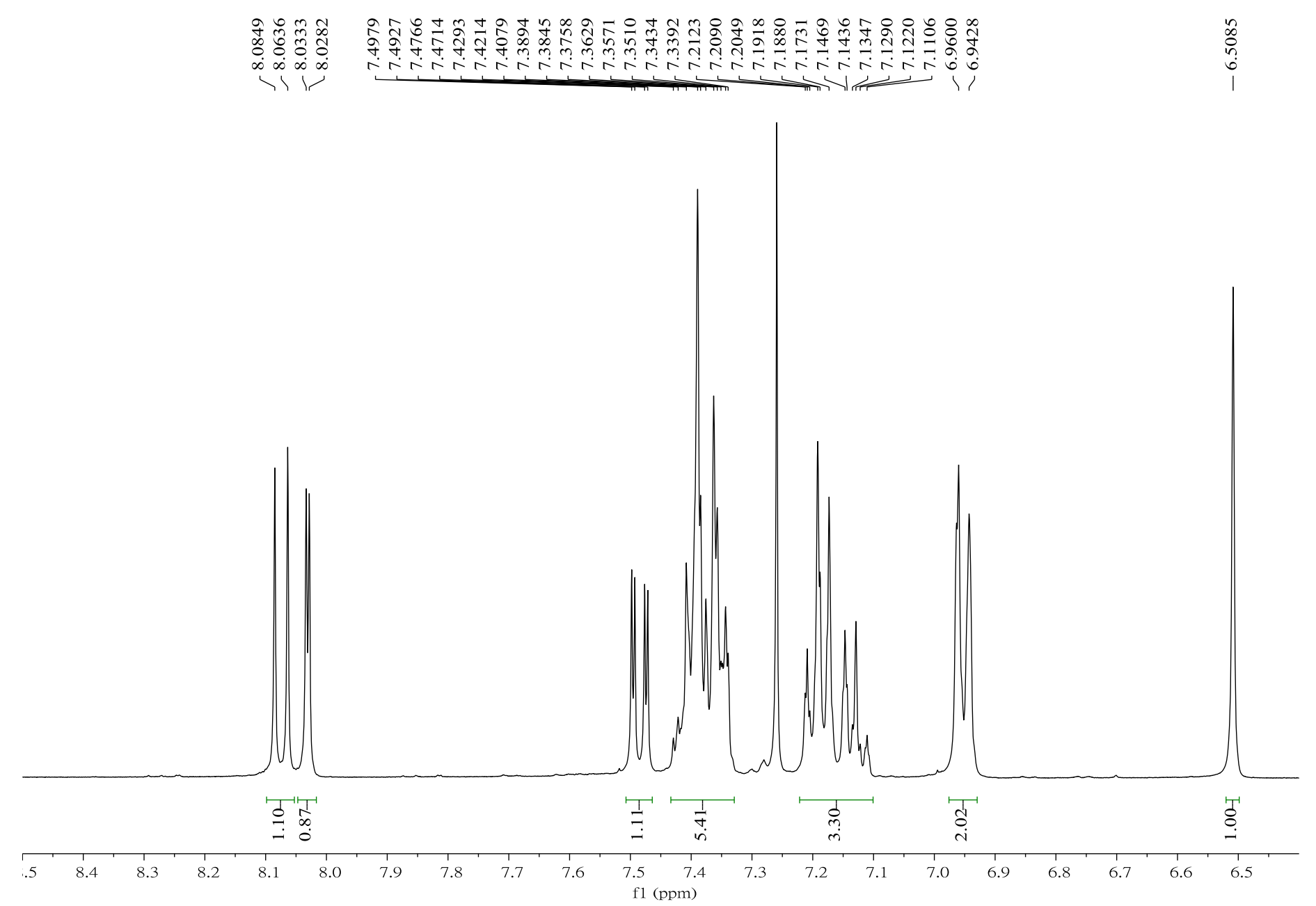

Expansion of ${ }^{1} \mathrm{H}$ NMR Spectrum $\left(400 \mathrm{MHz}\right.$ ) of compound $\mathbf{2} \mathbf{j}$ in $\mathrm{CDCl}_{3}$ 


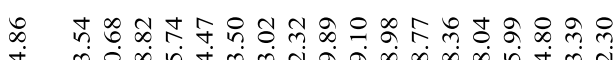

萡

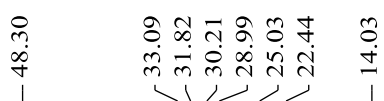

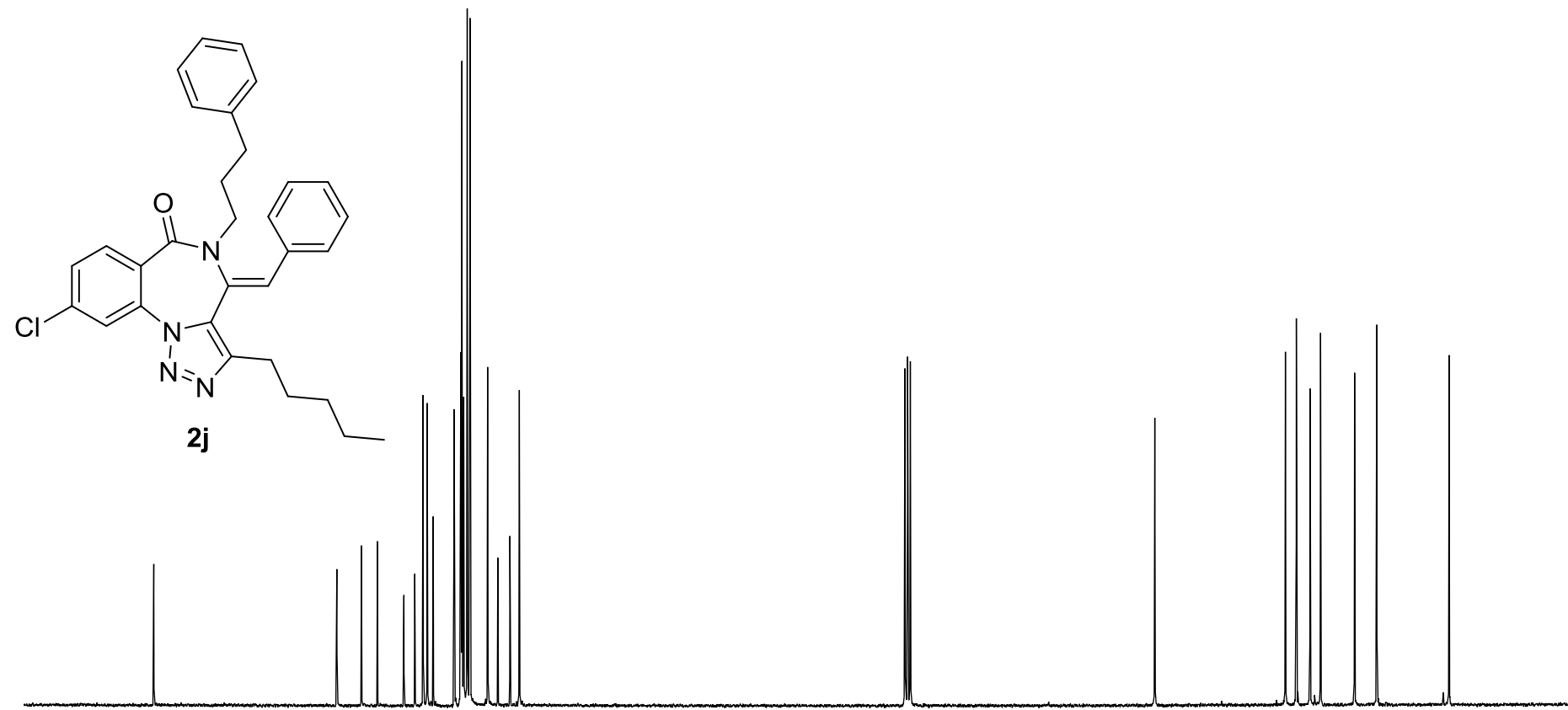

$80 \cdot 170 \cdot 160$

140 $130 \quad 120$

100

90

$80 \quad 70 \quad 60 \quad 50$

40 $20 \div \quad 10$

${ }^{13} \mathrm{C}$ NMR Spectrum (101 MHz) of compound $\mathbf{2 j}$ in $\mathrm{CDCl}_{3}$ 


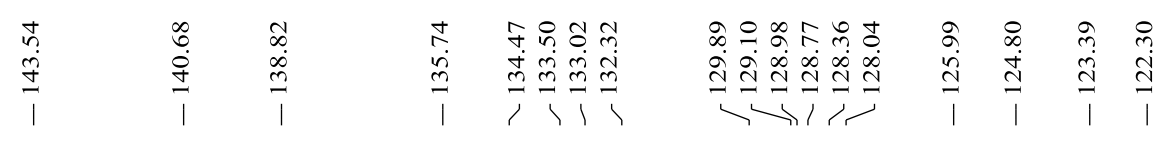

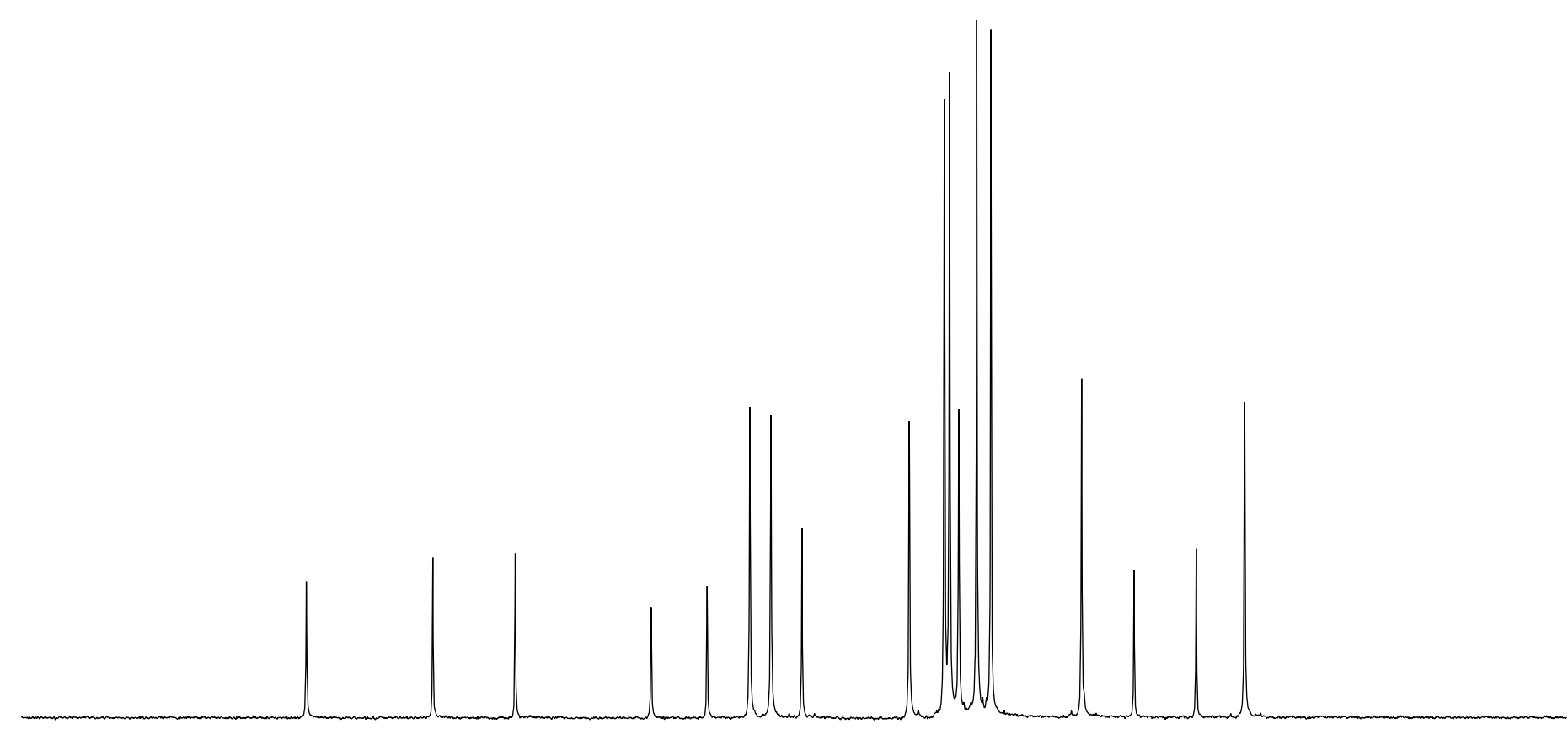

$\begin{array}{llllllllllllllllllllllllllllllllllllllllllllll}50 & 149 & 148 & 147 & 146 & 145 & 144 & 143 & 142 & 141 & 140 & 139 & 138 & 137 & 136 & 135 & 134 & 133 & 132 & 131 & 130 & 129 & 128 & 127 & 126 & 125 & 124 & 123 & 122 & 121 & 120 & 119 & 118 & 117 & 116 & 1\end{array}$ f1 (ppm)

Expansion of ${ }^{13} \mathrm{C}$ NMR Spectrum (101 MHz) of compound $\mathbf{2 j}$ in $\mathrm{CDCl}_{3}$ 
Display Report

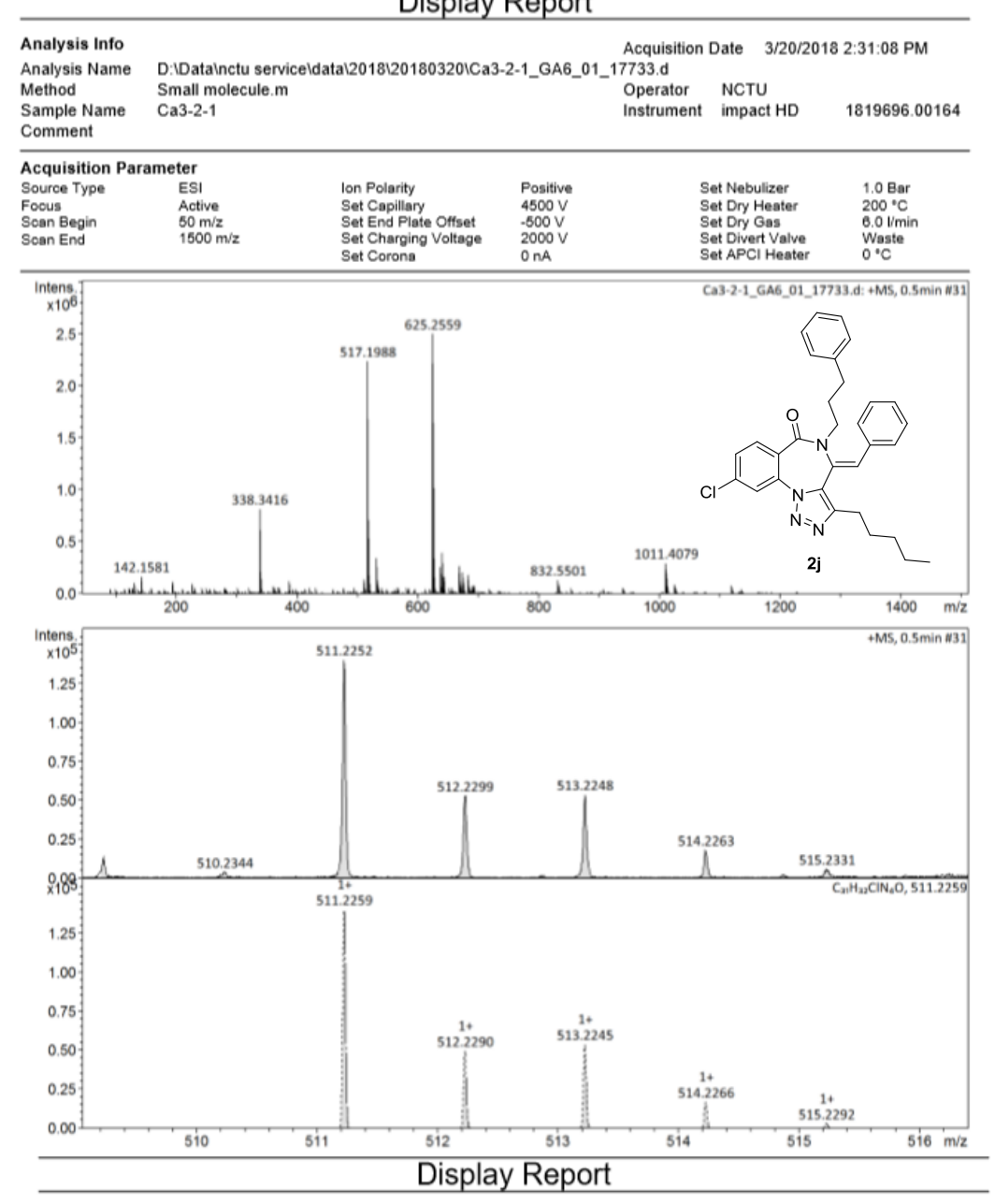

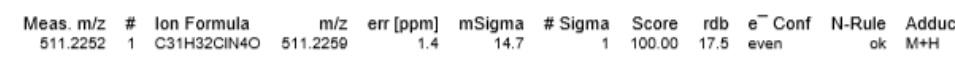

HRMS of compound $\mathbf{2} \mathbf{j}$ 


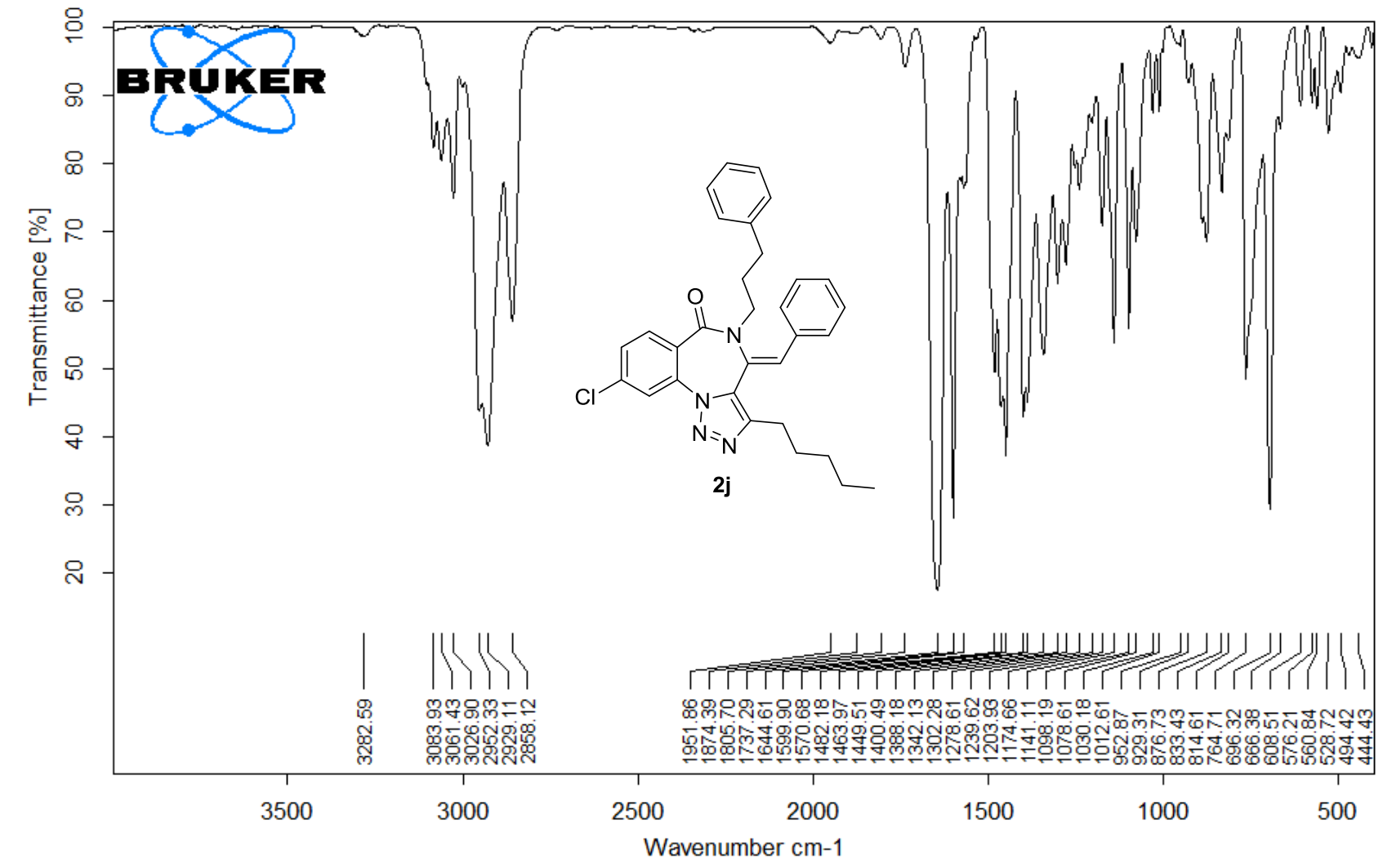

\begin{tabular}{|lcc|c|}
\hline D:IFTIR FILESI201808120180821IMIR_TR_DTGS_26122.0 MIR_TR_DTGS_26122 $\quad$ Instrument type and / or accessory & $8 / 21 / 2018$ \\
\hline
\end{tabular}

Page 1/1

IR of compound $\mathbf{2 j}$ 


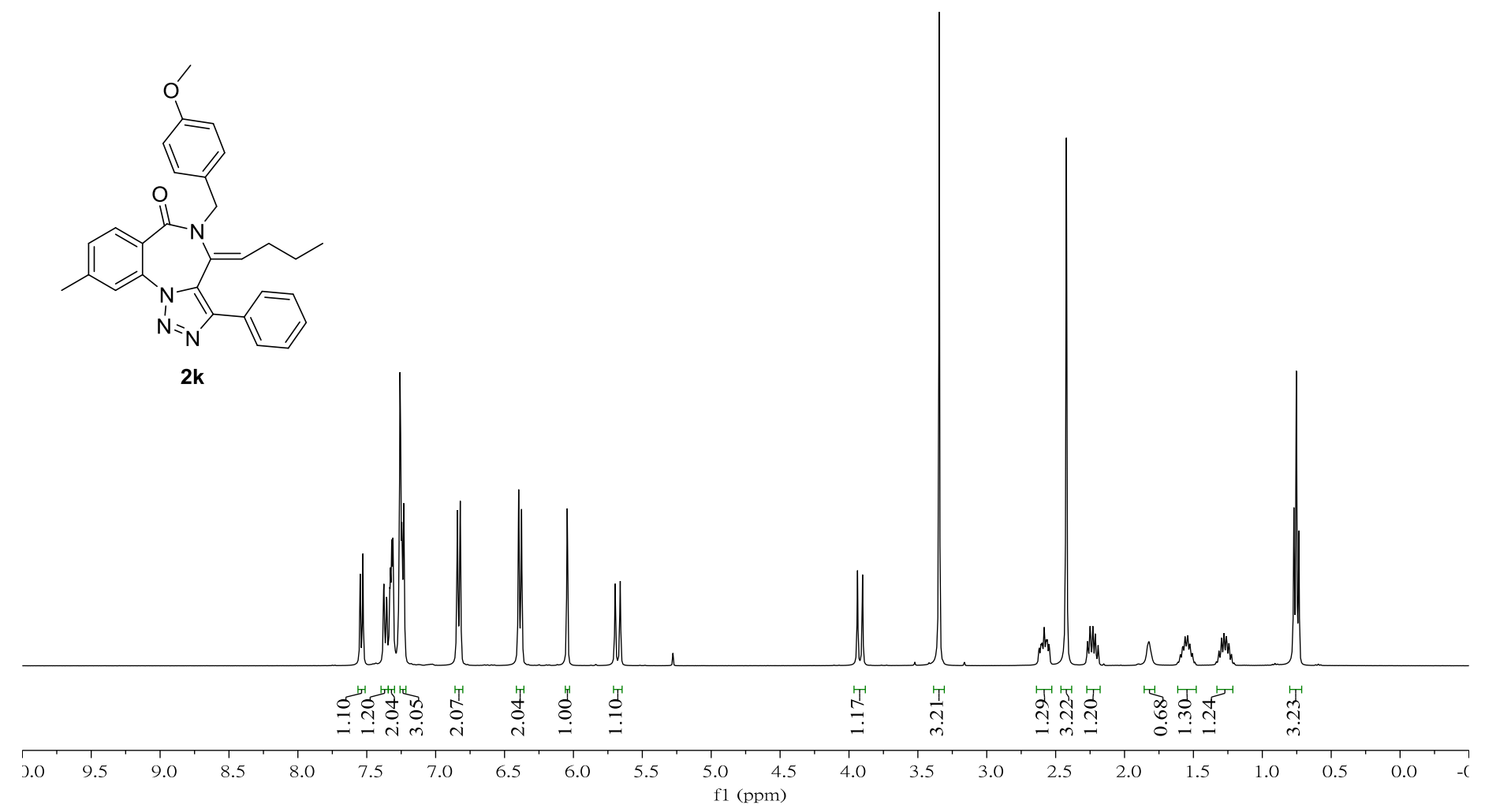

${ }^{1} \mathrm{H}$ NMR Spectrum (400 MHz) of compound $\mathbf{2 k}$ in $\mathrm{CDCl}_{3}$ 


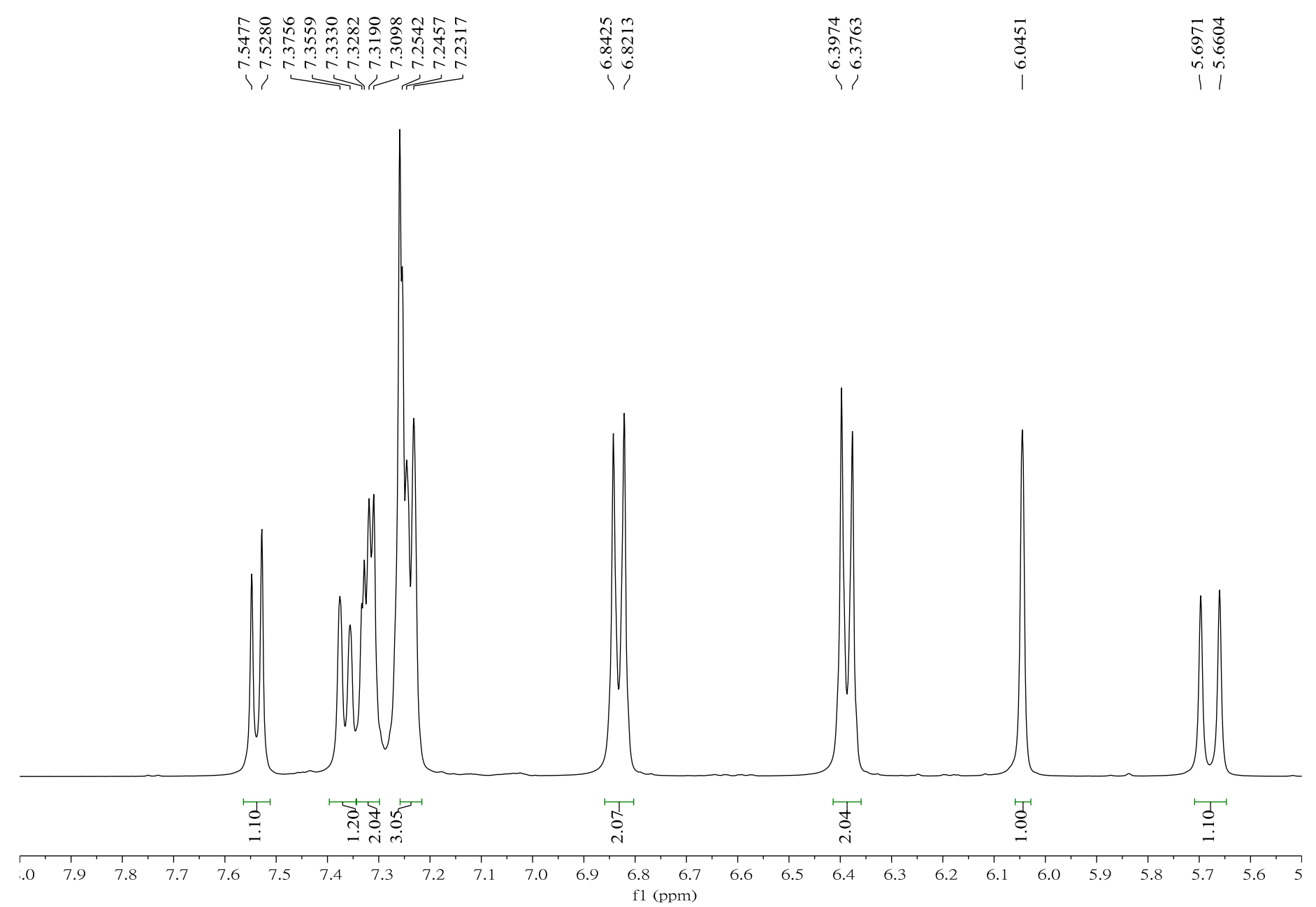

Expansion of ${ }^{1} \mathrm{H}$ NMR Spectrum (400 MHz) of compound $\mathbf{2 k}$ in $\mathrm{CDCl}_{3}$ 


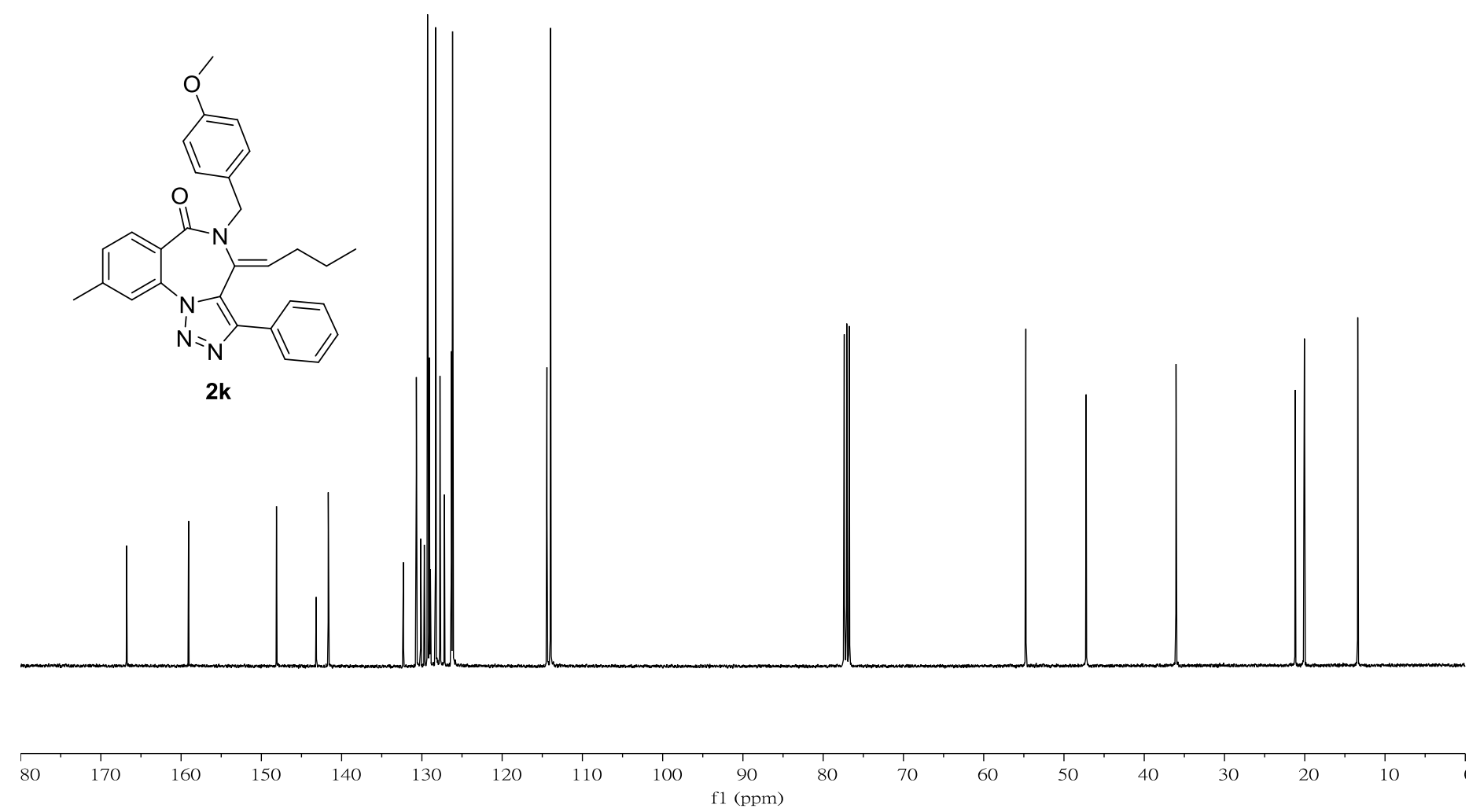

${ }^{13} \mathrm{C}$ NMR Spectrum (101 MHz) of compound $\mathbf{2 k}$ in $\mathrm{CDCl}_{3}$ 


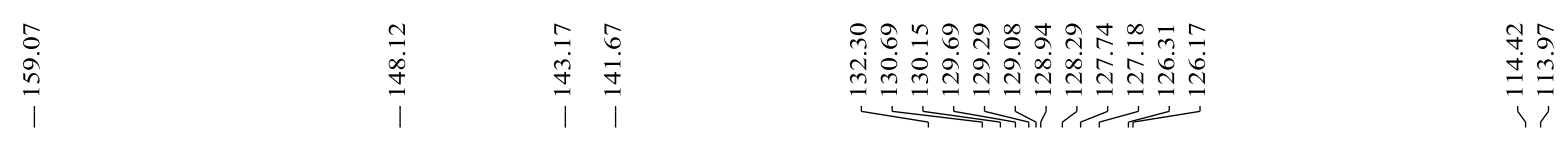

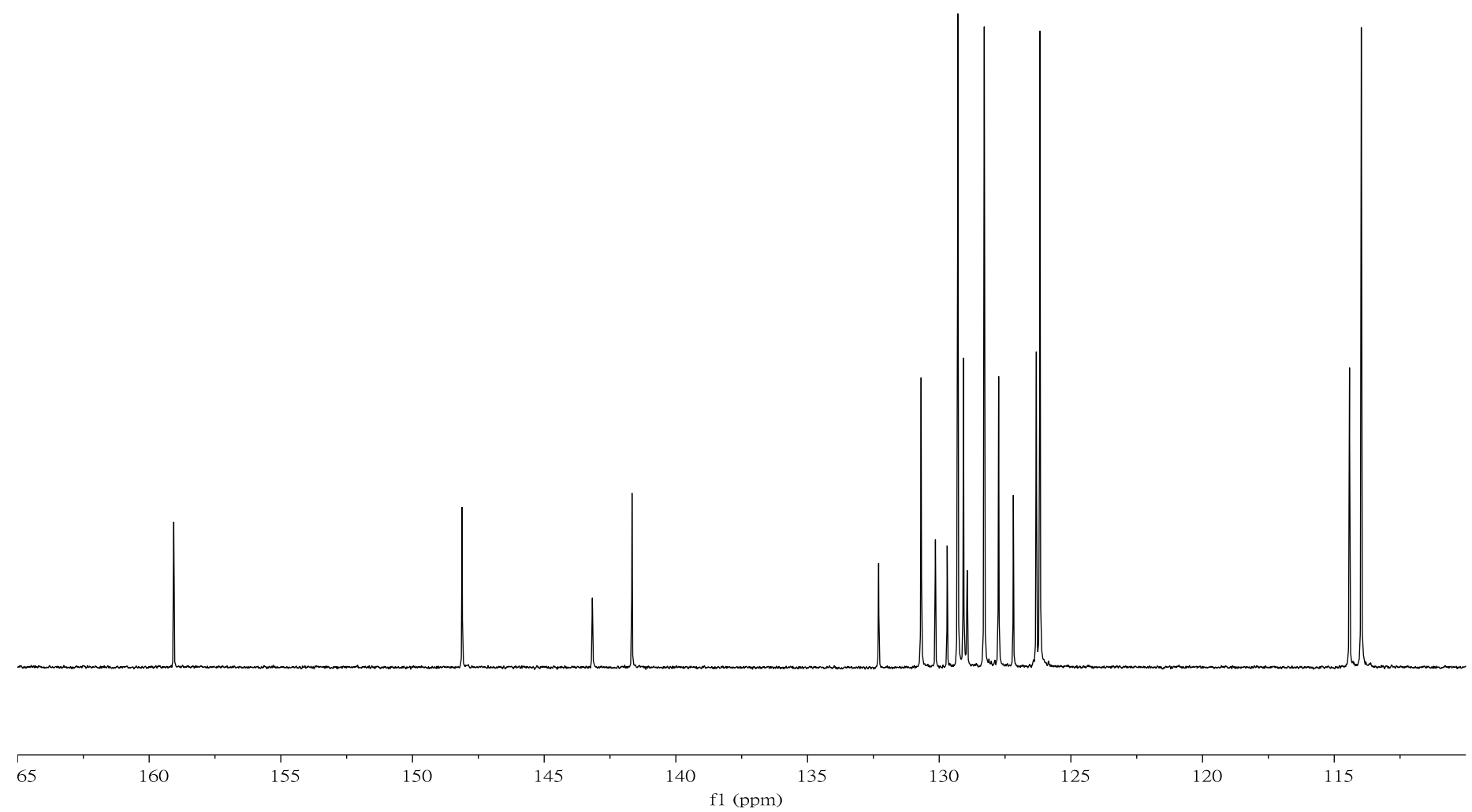

Expansion of ${ }^{13} \mathrm{C}$ NMR Spectrum $(101 \mathrm{MHz})$ of compound $\mathbf{2 k}$ in $\mathrm{CDCl}_{3}$ 
Display Report

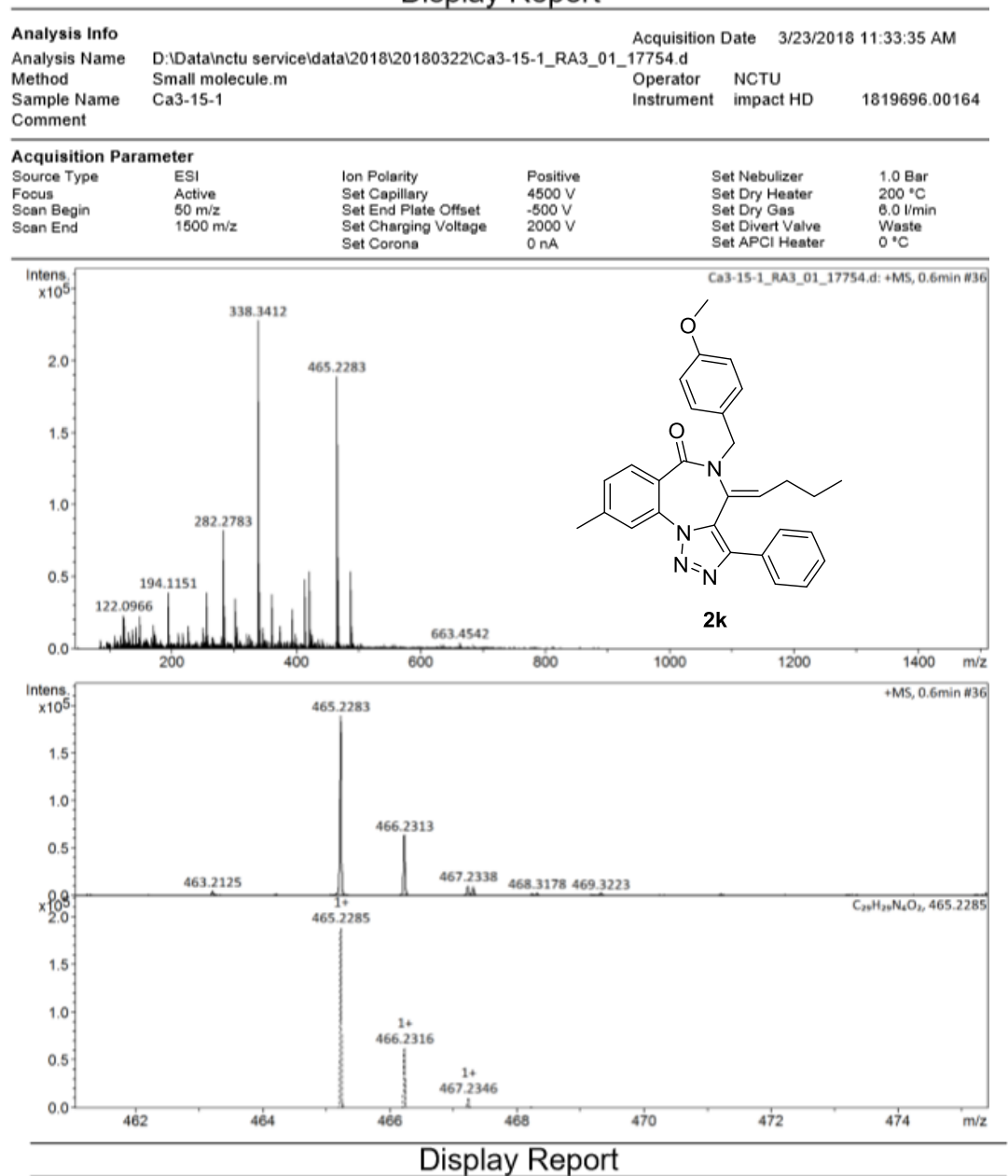

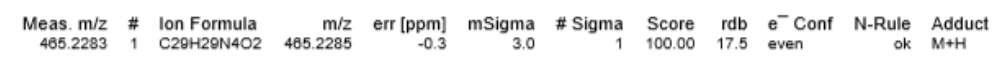

HRMS of compound $\mathbf{2 k}$ 


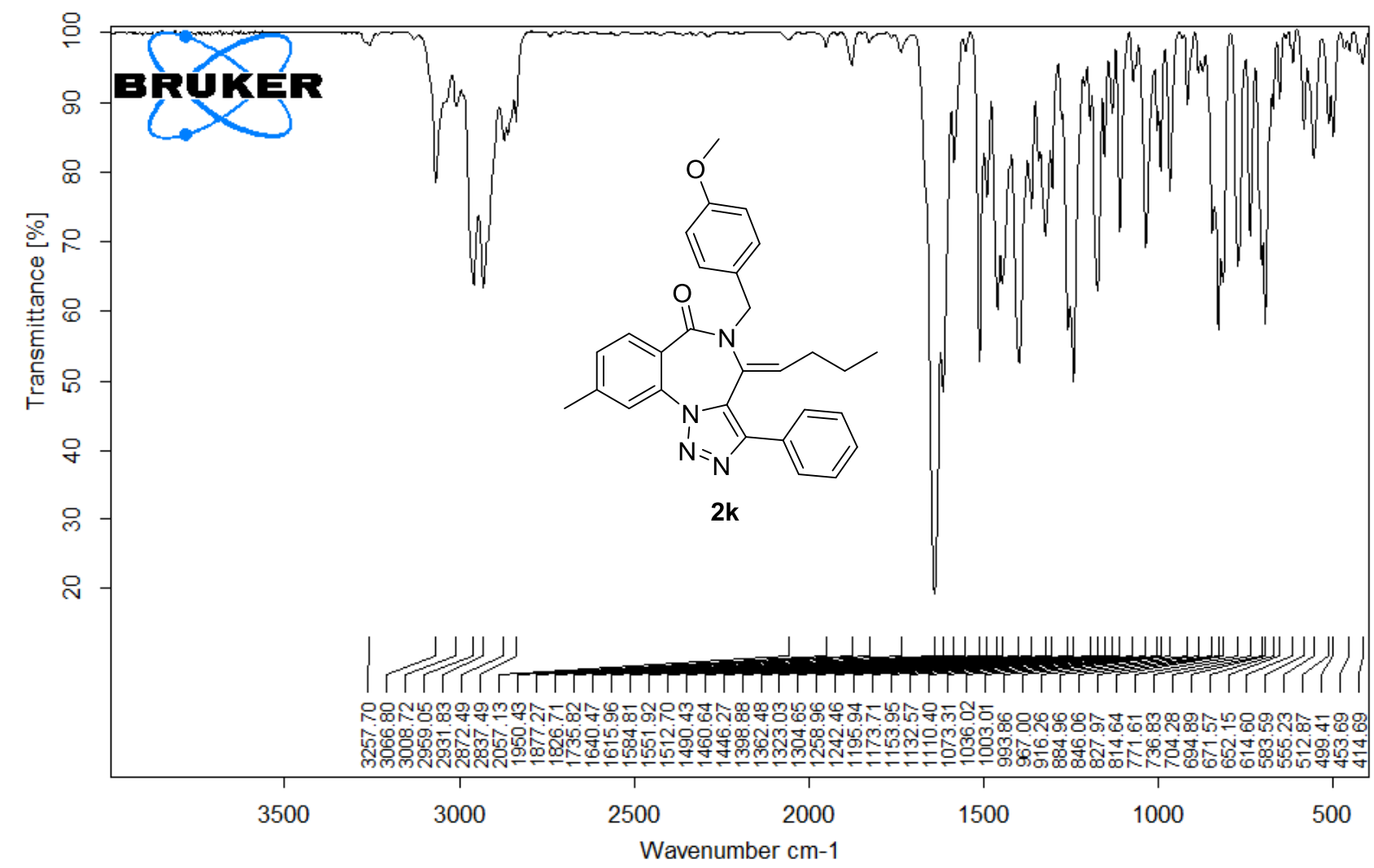

\begin{tabular}{|lcl|l|}
\hline D:IFTIR FILESI201808120180821MIR_TR_DTGS_26123.0 $\quad$ MIR_TR_DTGS_26123 $\quad$ Instrument type and / or accessory & $8 / 21 / 2018$ \\
\hline
\end{tabular}

Page 1/1

IR of compound $\mathbf{2 k}$ 


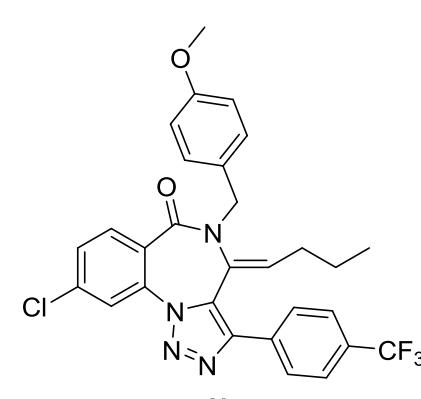

21

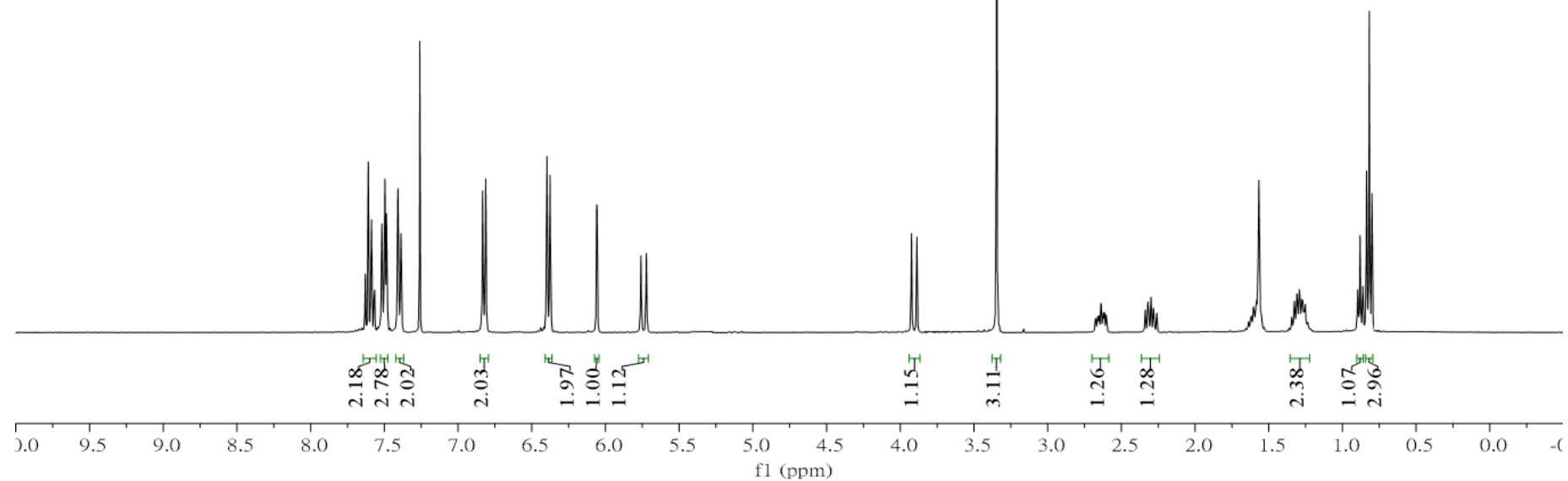

${ }^{1} \mathrm{H}$ NMR Spectrum (400 MHz) of compound $2 \mathbf{l}$ in $\mathrm{CDCl}_{3}$ 


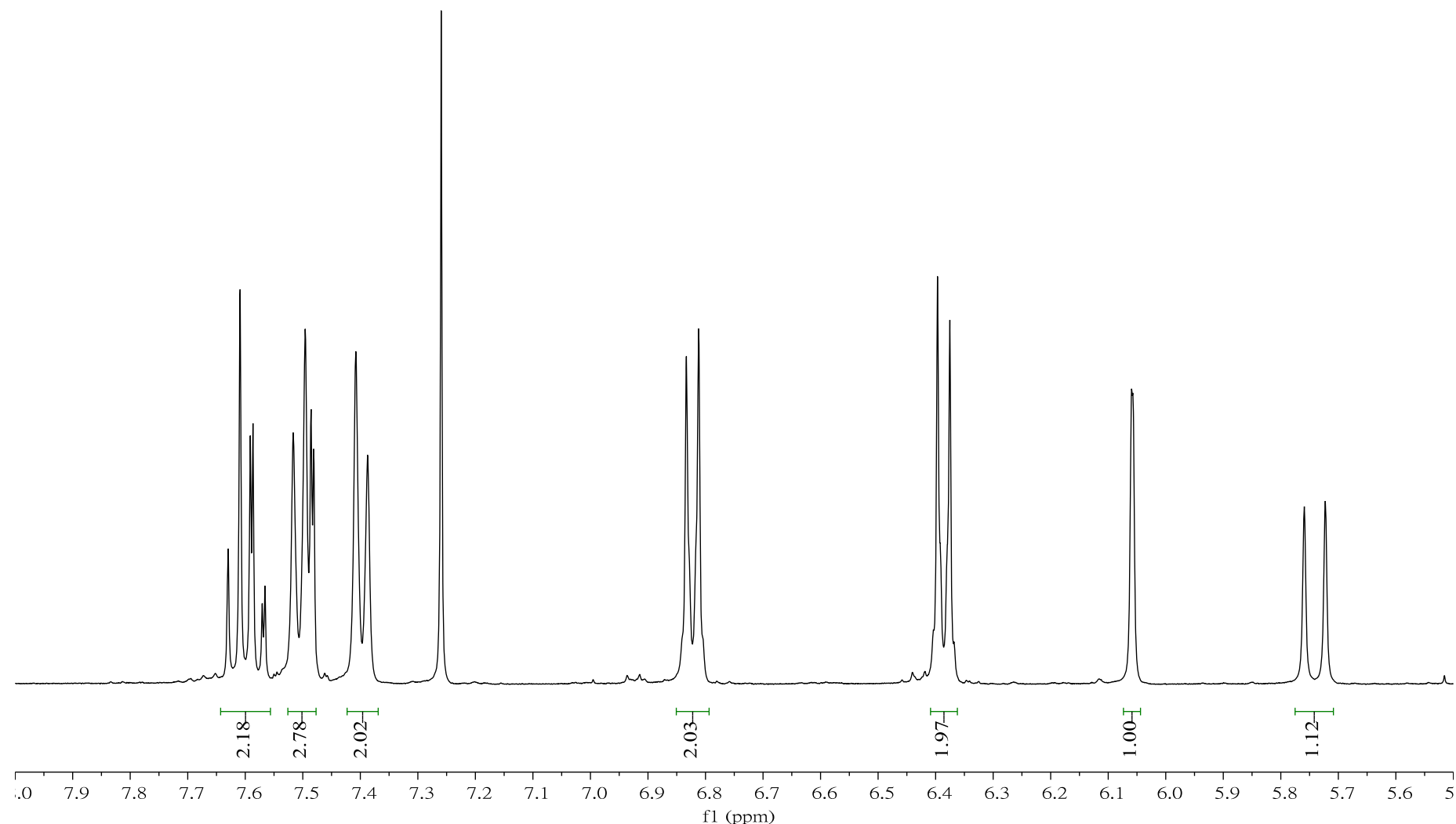

Expansion of ${ }^{1} \mathrm{H}$ NMR Spectrum $\left(400 \mathrm{MHz}\right.$ ) of compound $2 \mathbf{l}$ in $\mathrm{CDCl}_{3}$ 


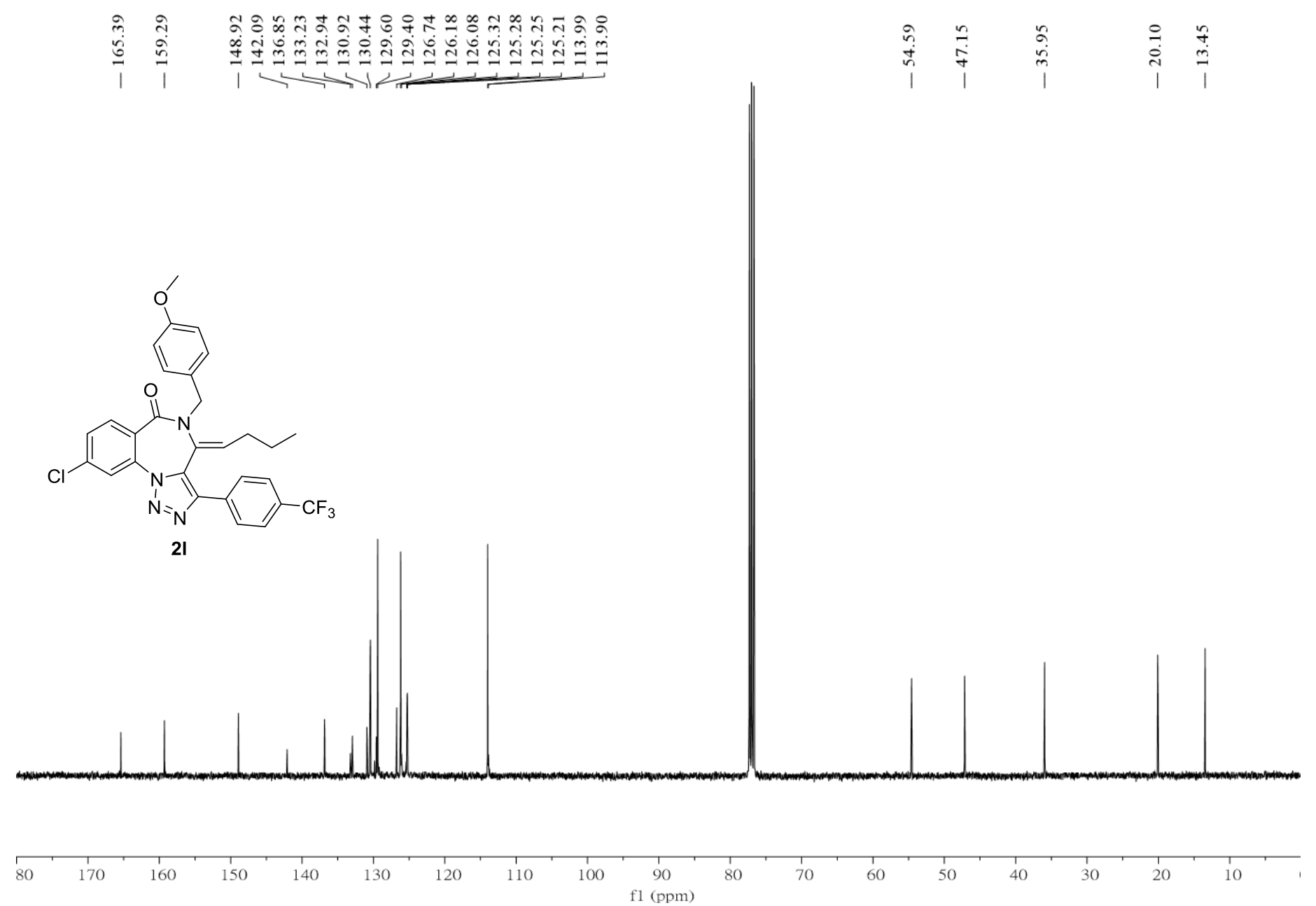

${ }^{13} \mathrm{C}$ NMR Spectrum (101 MHz) of compound $2 \mathbf{l}$ in $\mathrm{CDCl}_{3}$ 


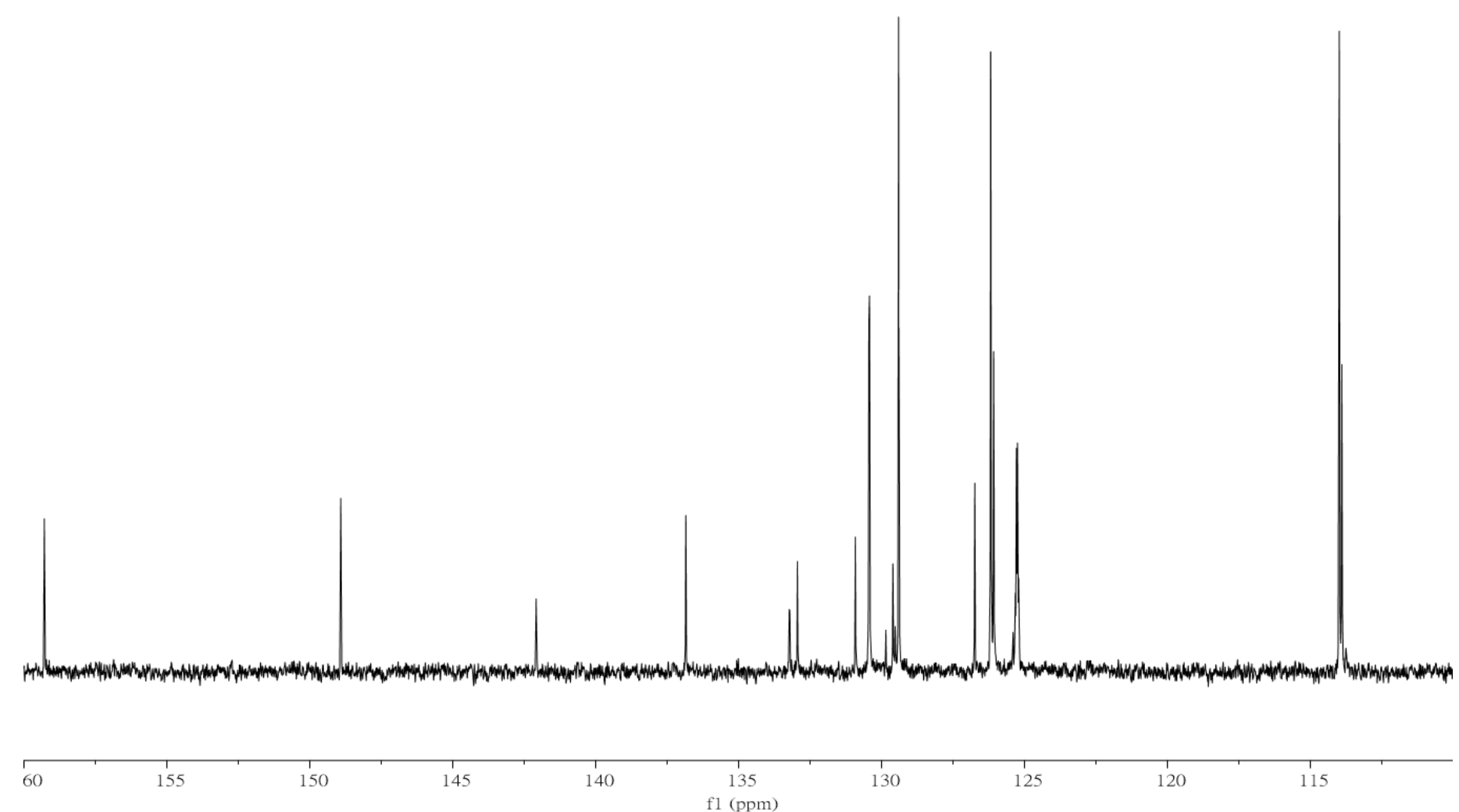

Expansion of ${ }^{13} \mathrm{C}$ NMR Spectrum (101 MHz) of compound $2 \mathrm{l}$ in $\mathrm{CDCl}_{3}$ 
Display Report

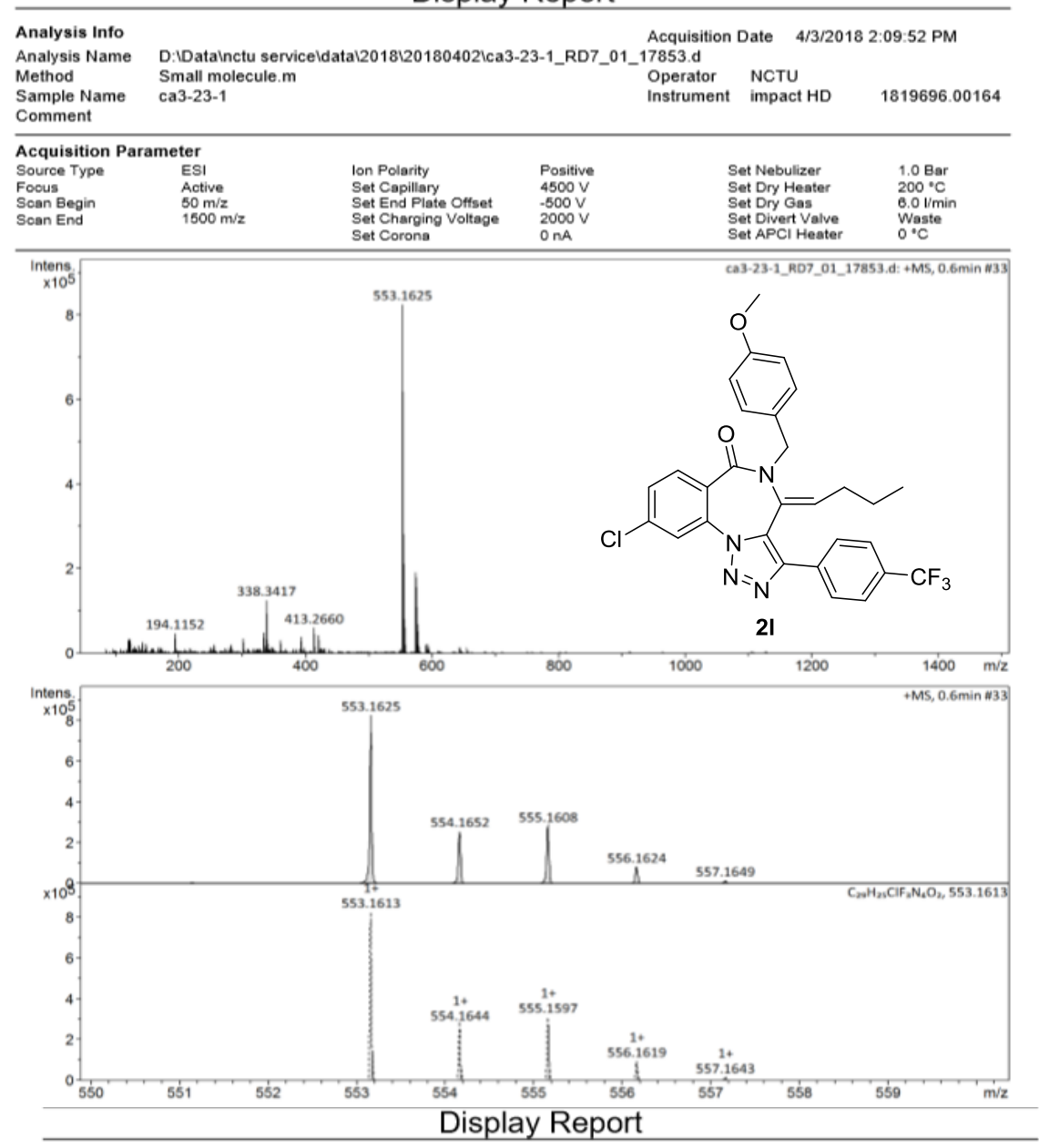

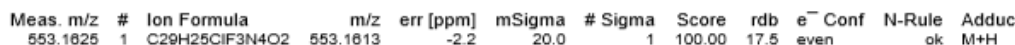

HRMS of compound 21 


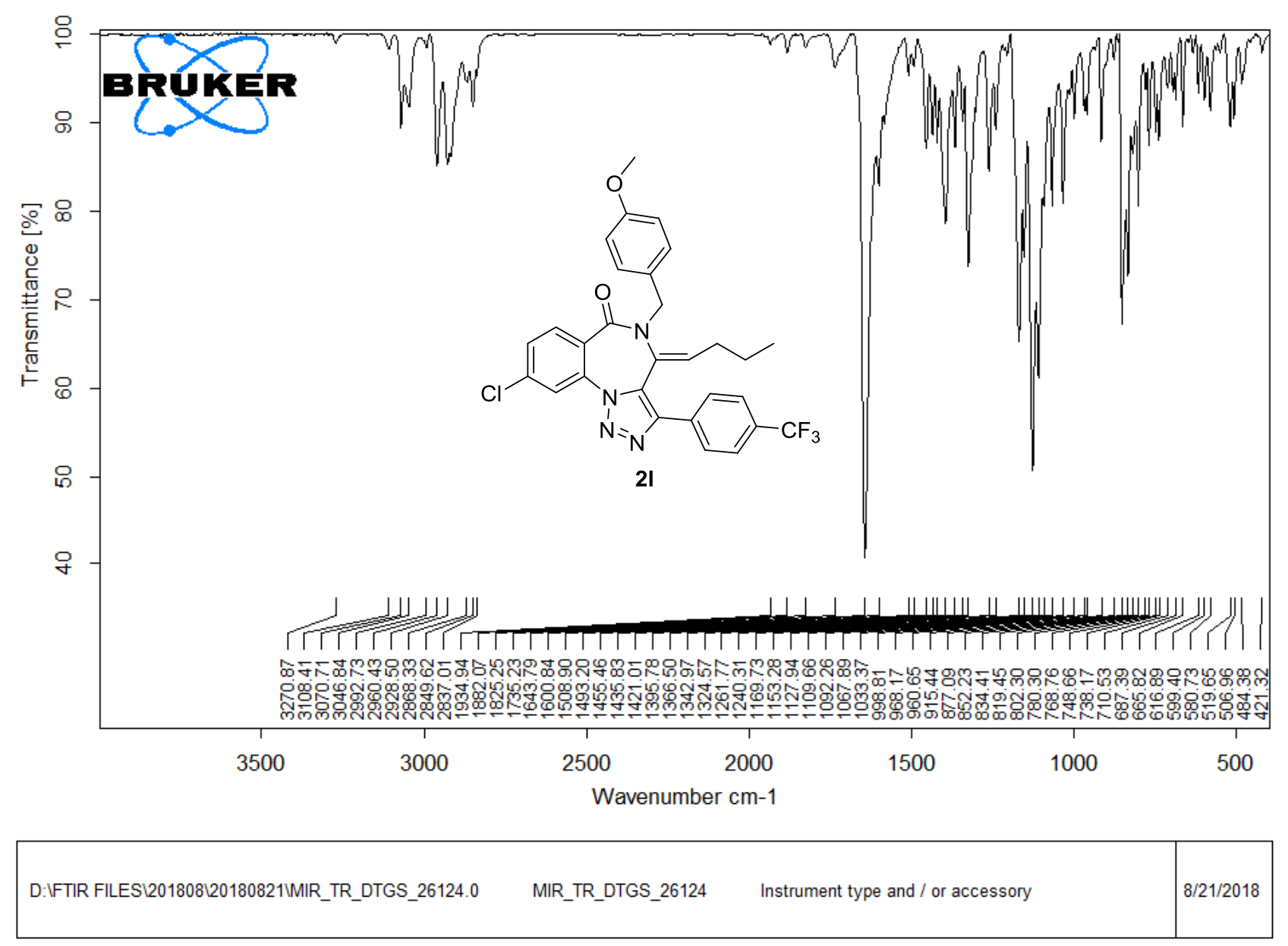

Page 1/1

IR of compound 2l 


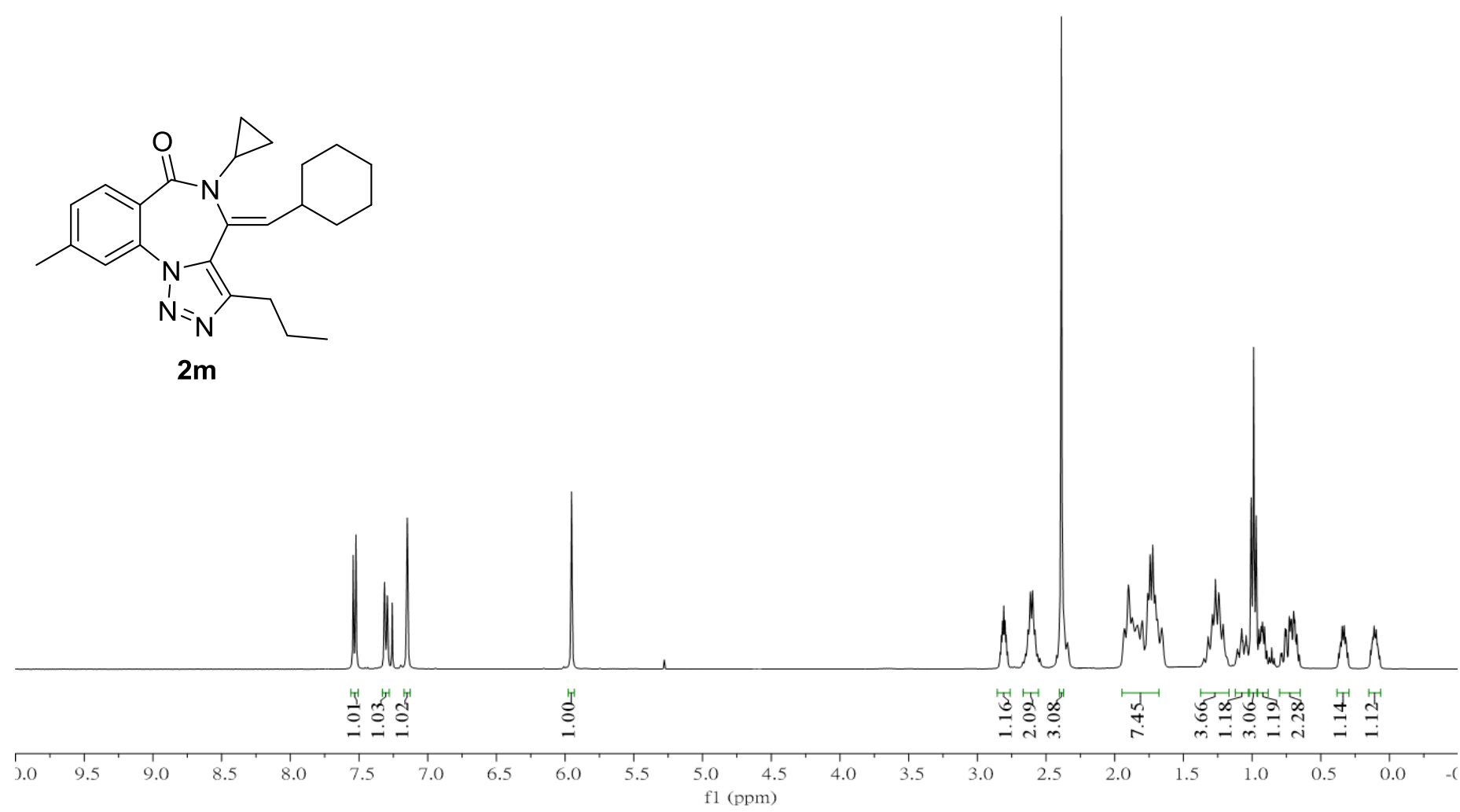

${ }^{1} \mathrm{H}$ NMR Spectrum (400 MHz) of compound $\mathbf{2 m}$ in $\mathrm{CDCl}_{3}$ 


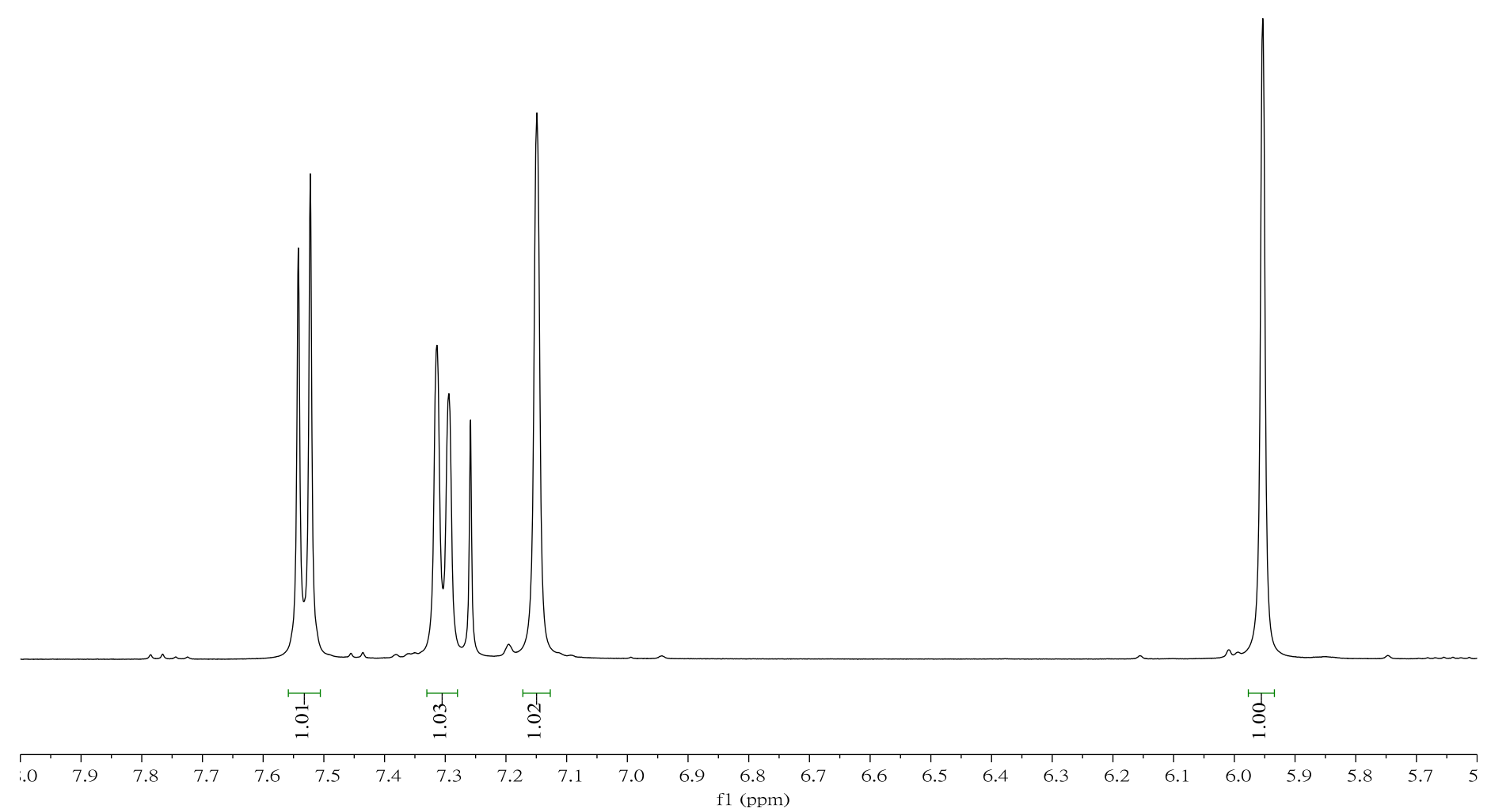

Expansion of ${ }^{1} \mathrm{H}$ NMR Spectrum (400 MHz) of compound $\mathbf{2 m}$ in $\mathrm{CDCl}_{3}$ 


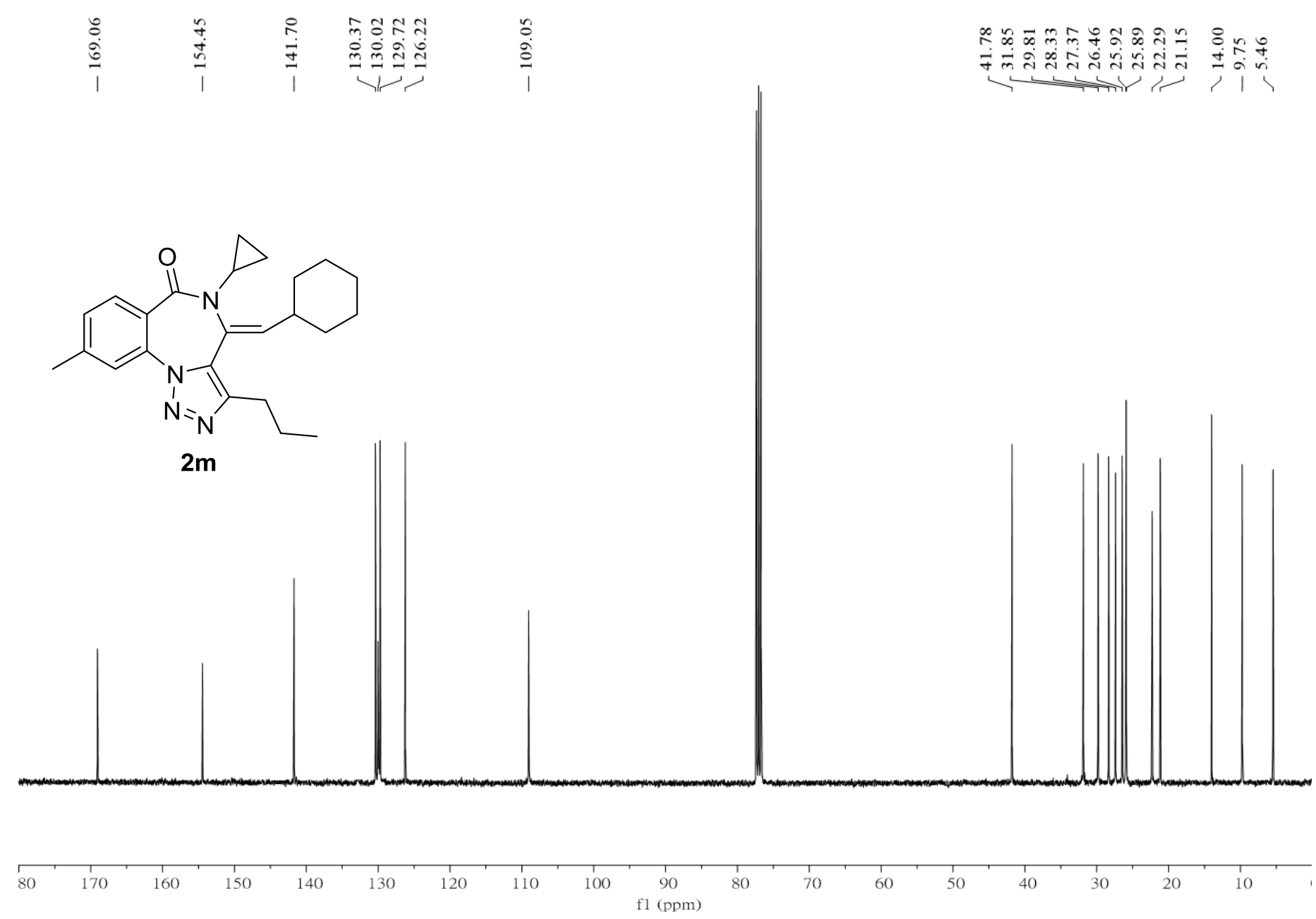

${ }^{13} \mathrm{C}$ NMR Spectrum (101 MHz) of compound $\mathbf{2 m}$ in $\mathrm{CDCl}_{3}$ 

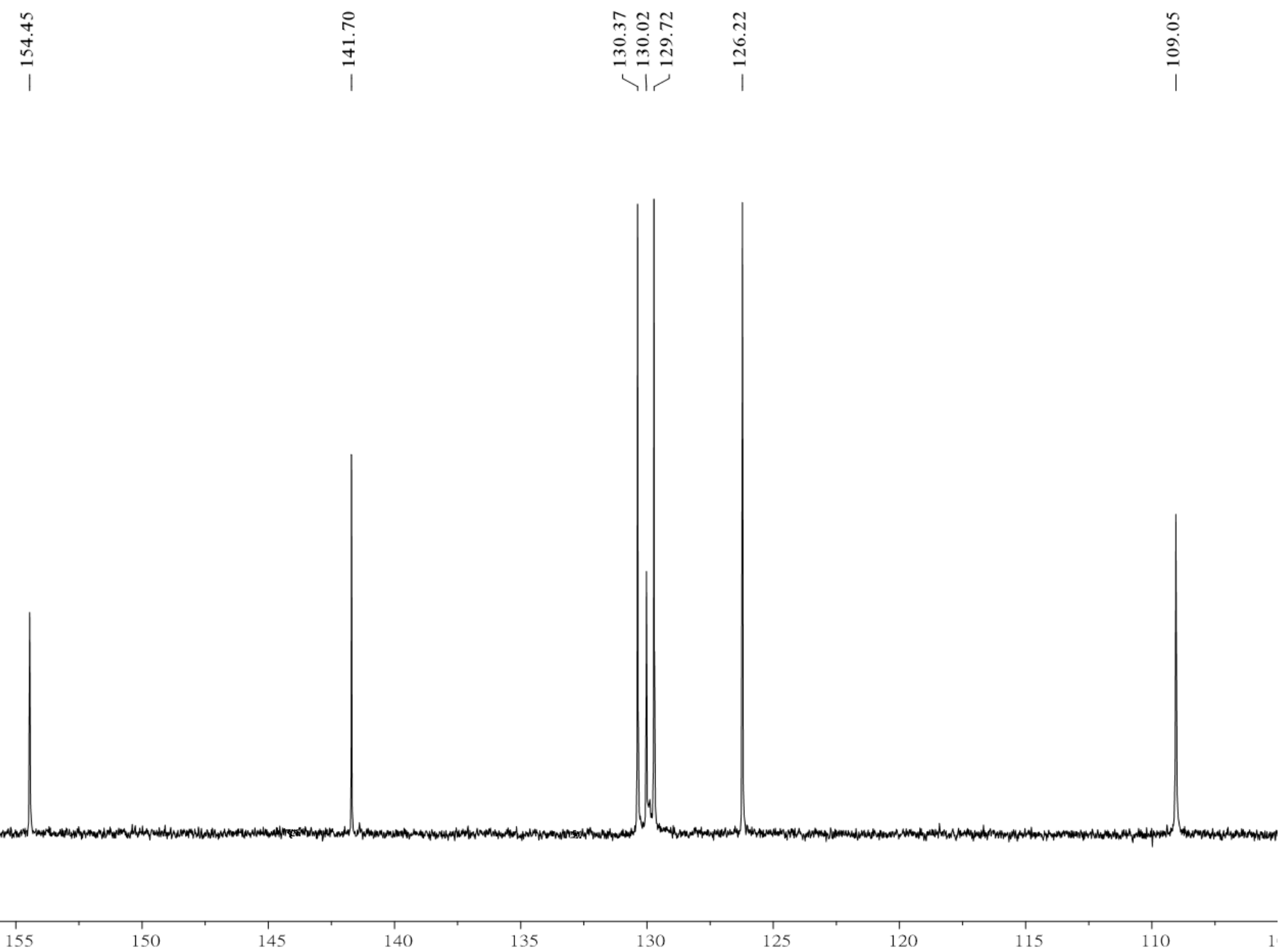

140

13

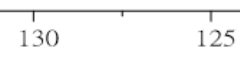

120

115

110

Expansion of ${ }^{13} \mathrm{C}$ NMR Spectrum $\left(101 \mathrm{MHz}\right.$ ) of compound $\mathbf{2 m}$ in $\mathrm{CDCl}_{3}$ 
Display Report

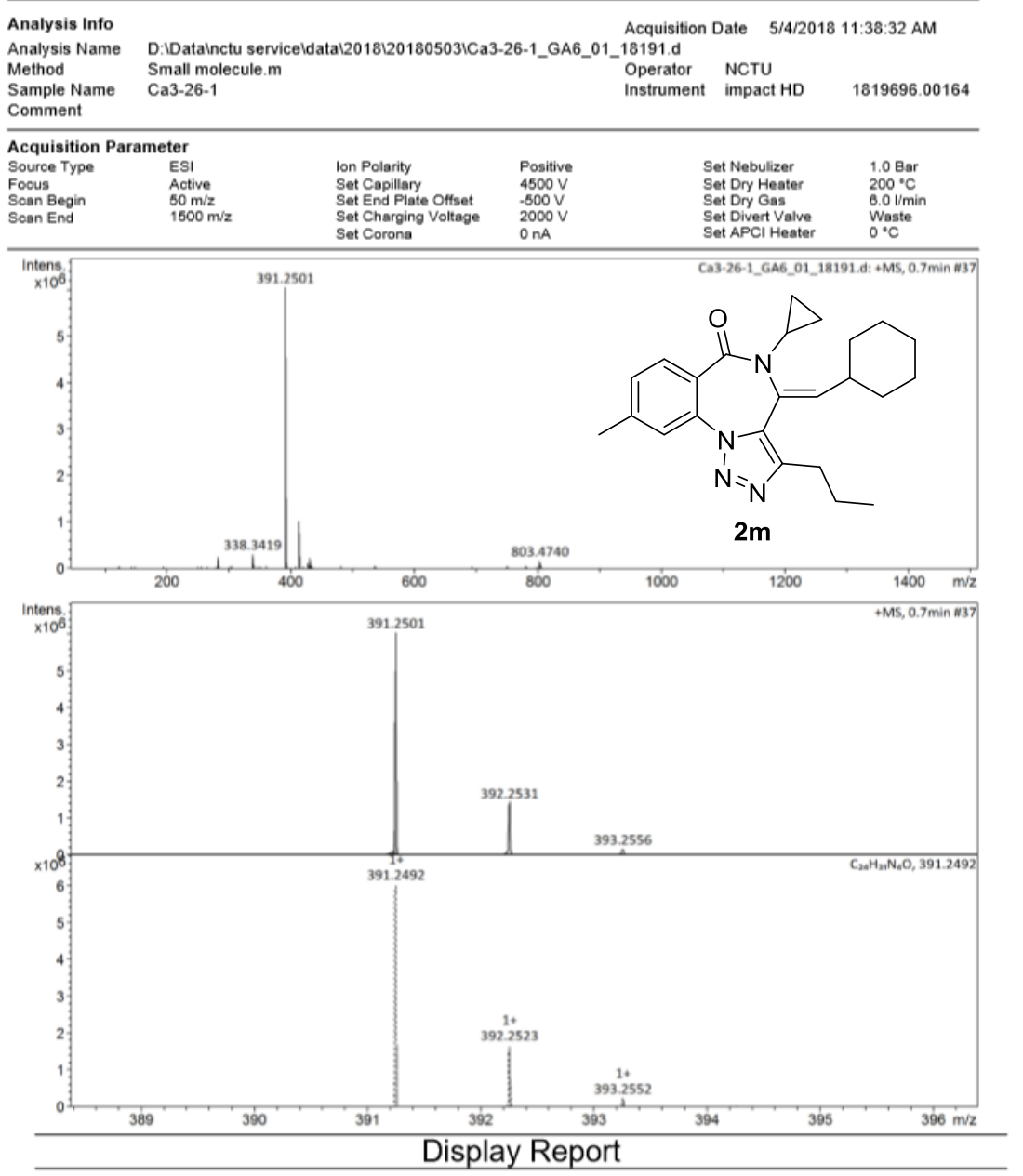

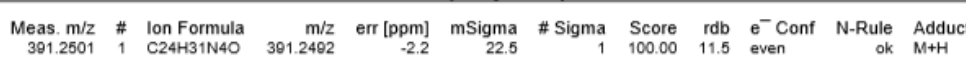

HRMS of compound $\mathbf{2 m}$ 


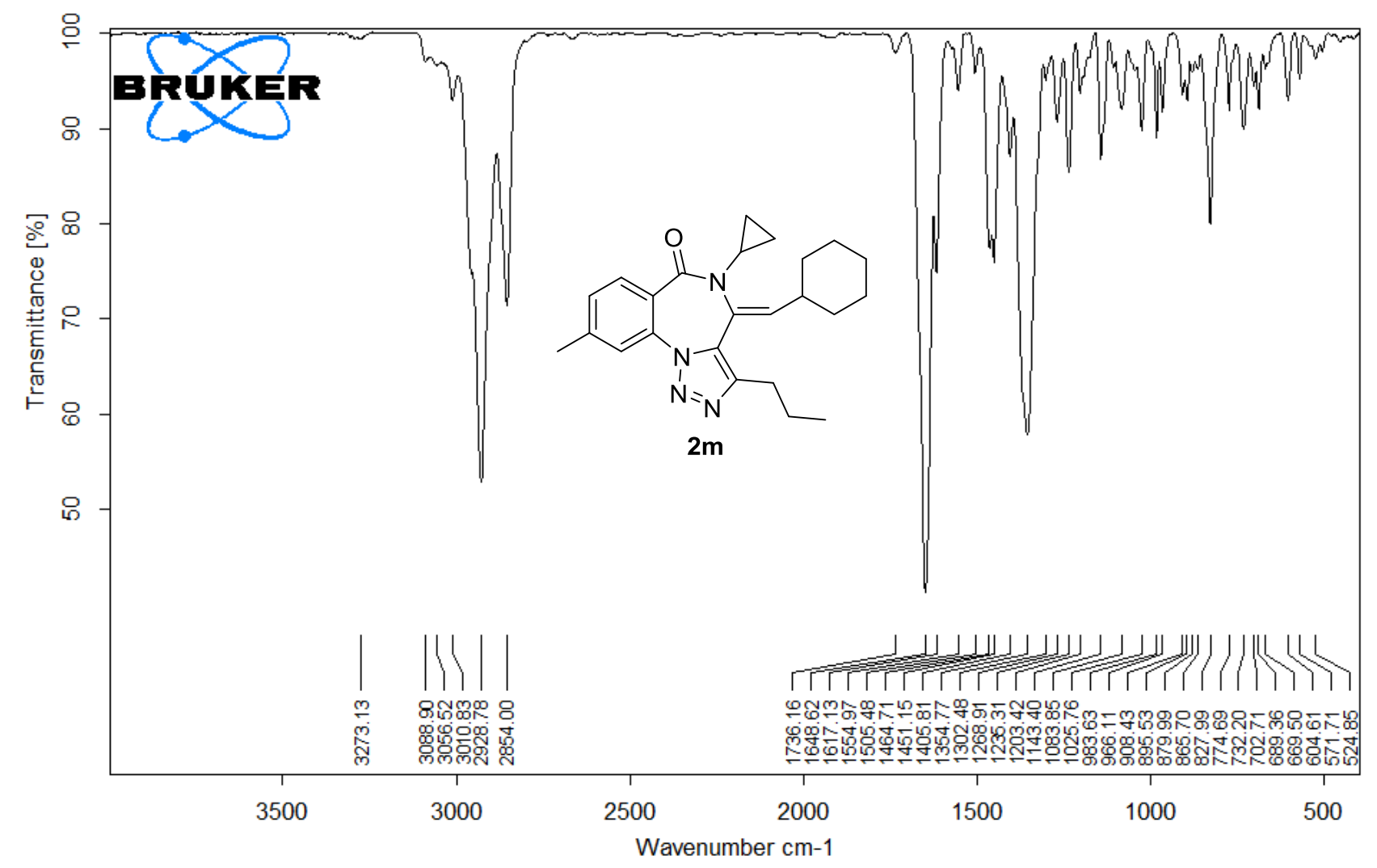

\begin{tabular}{|lcc|c|}
\hline D:IFTIR FILESI201808120180821MIR_TR_DTGS_26125.0 MIR_TR_DTGS_26125 $\quad$ Instrument type and / or accessory & $8 / 21 / 2018$ \\
\hline
\end{tabular}

Page 1/1

IR of compound $\mathbf{2 m}$ 


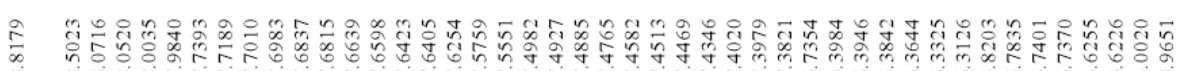

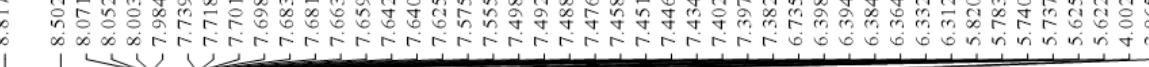
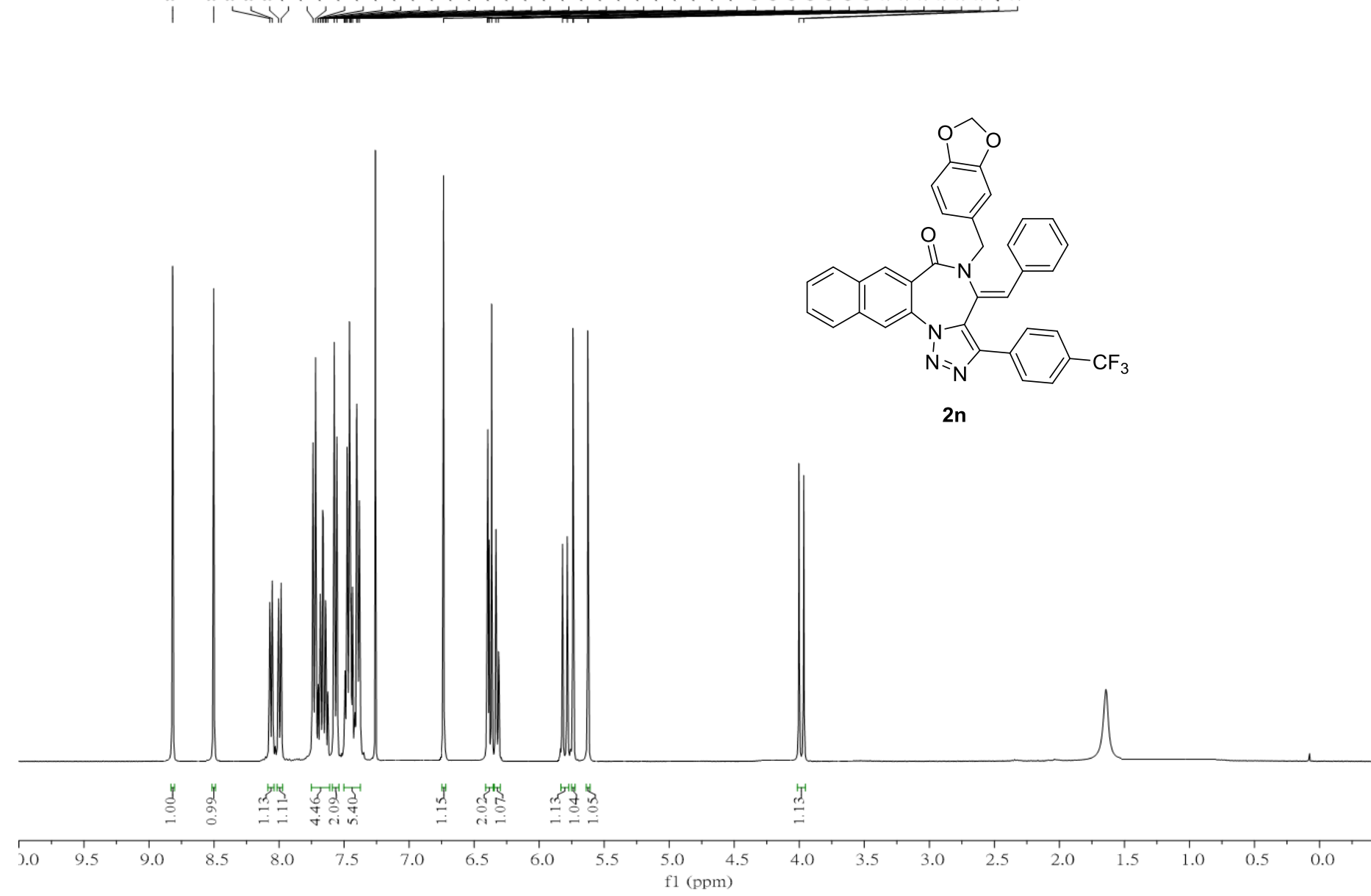

${ }^{1} \mathrm{H}$ NMR Spectrum (400 MHz) of compound $\mathbf{2 n}$ in $\mathrm{CDCl}_{3}$ 

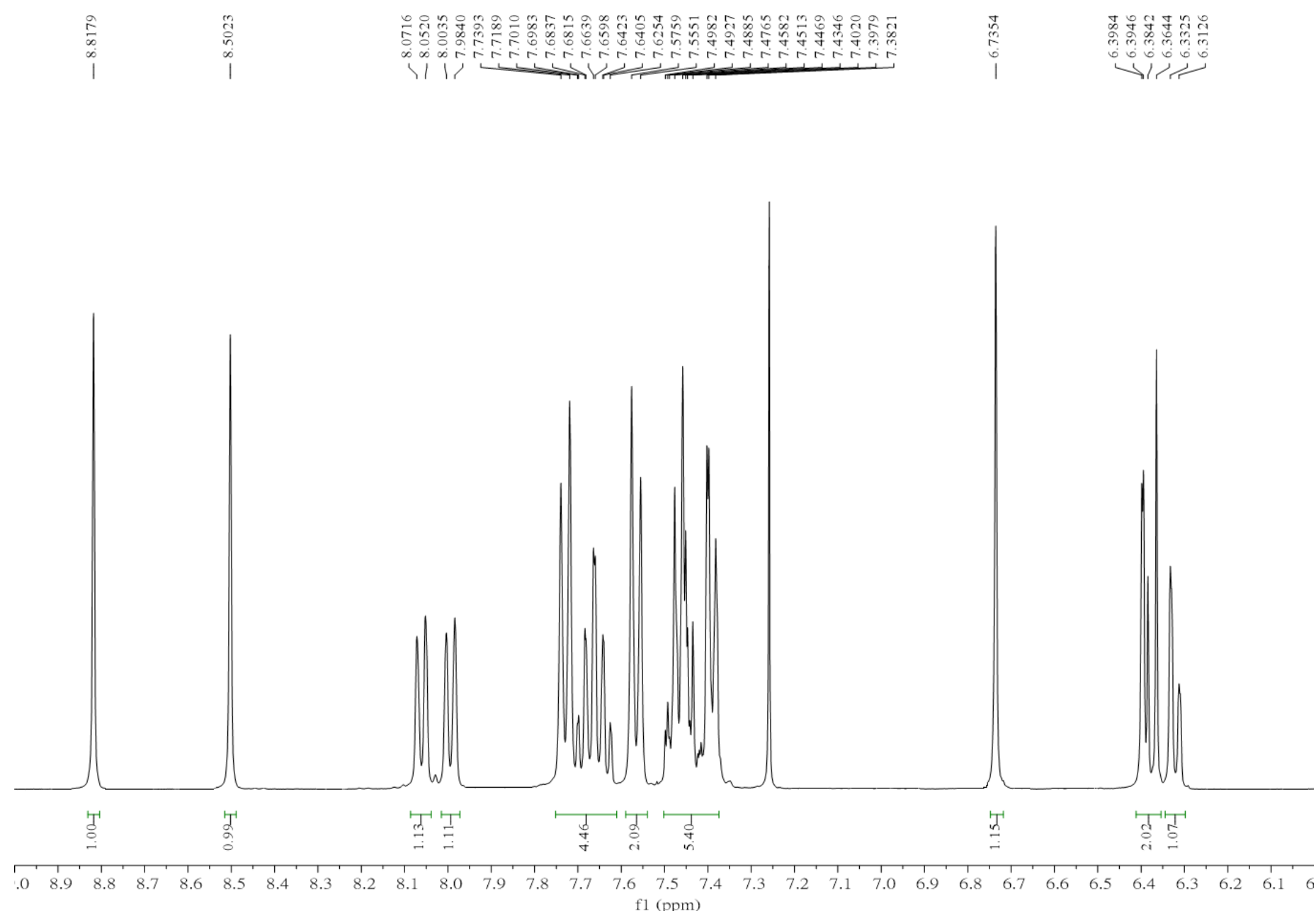

Expansion of ${ }^{1} \mathrm{H}$ NMR Spectrum (400 MHz) of compound $\mathbf{2 n}$ in $\mathrm{CDCl}_{3}$ 


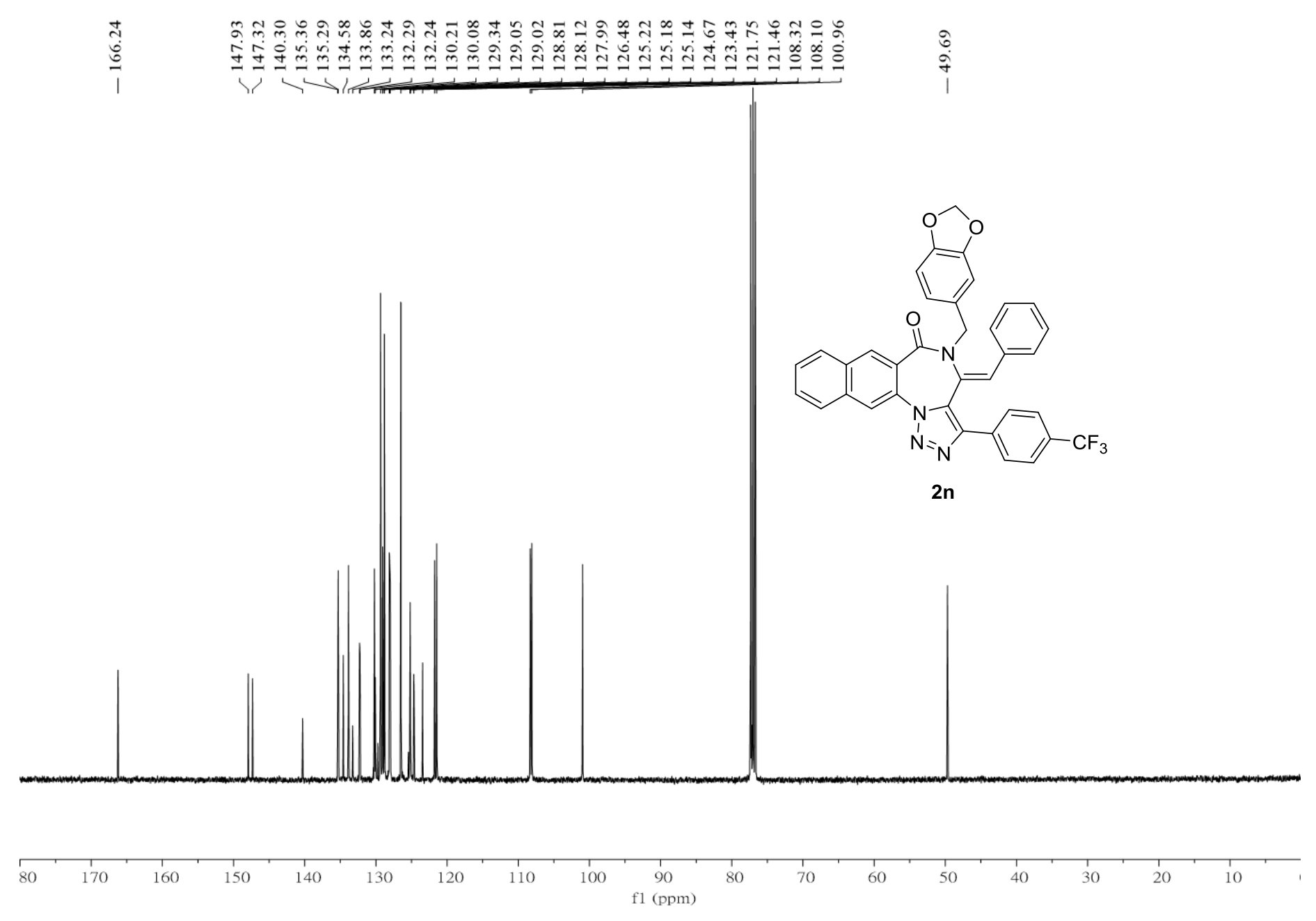

${ }^{13} \mathrm{C}$ NMR Spectrum (101 MHz) of compound $\mathbf{2 n}$ in $\mathrm{CDCl}_{3}$ 

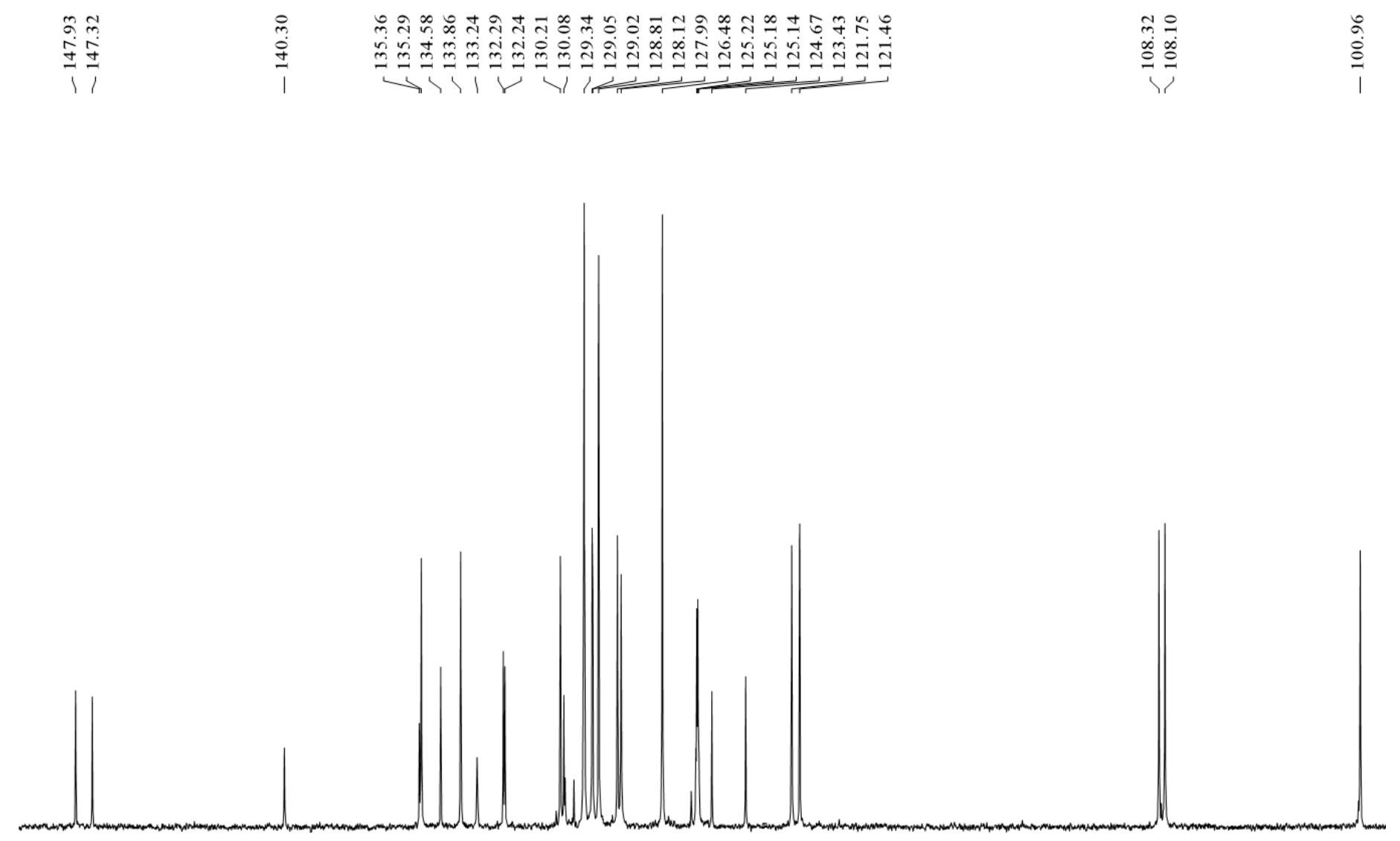

50 140 135 130 125
f1 $(\mathrm{ppm})$ 120 115 110 105

Expansion of ${ }^{13} \mathrm{C}$ NMR Spectrum $(101 \mathrm{MHz})$ of compound $\mathbf{2 n}$ in $\mathrm{CDCl}_{3}$ 
Display Report

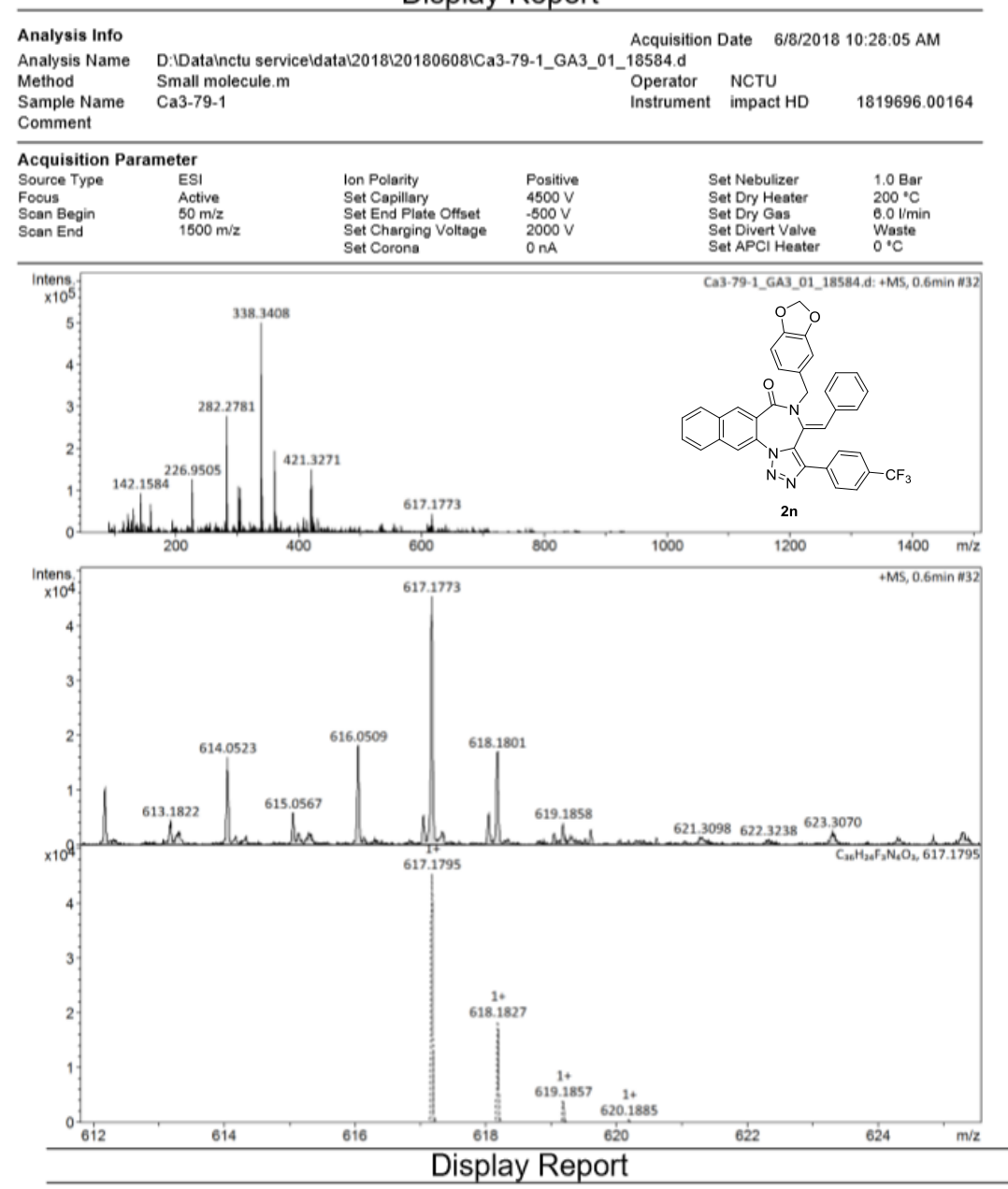

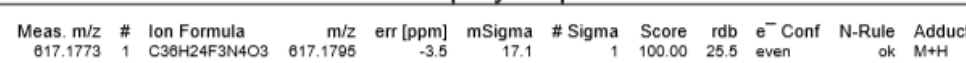

HRMS of compound 2n 


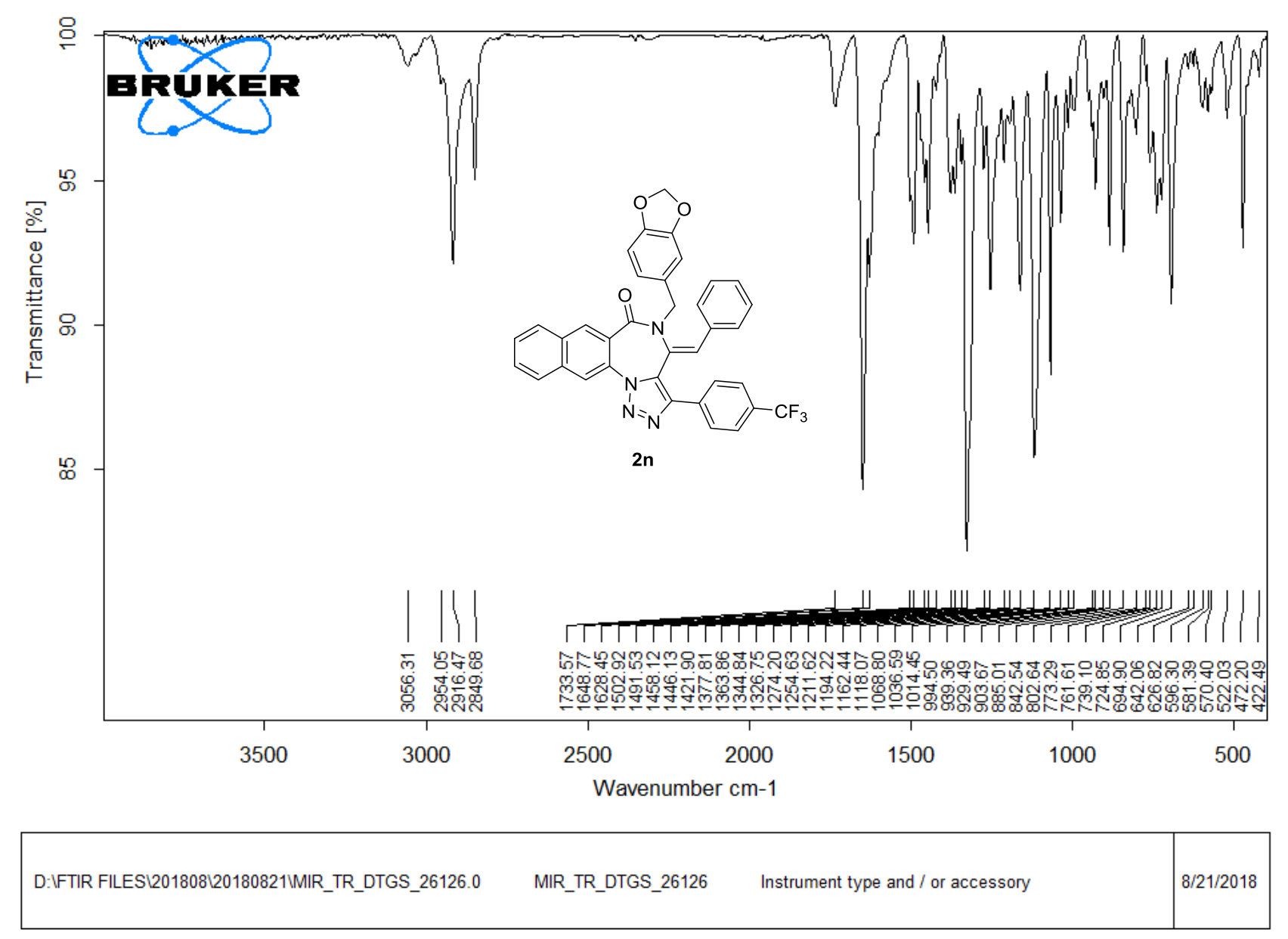

Page 1/1

IR of compound $\mathbf{2 n}$ 


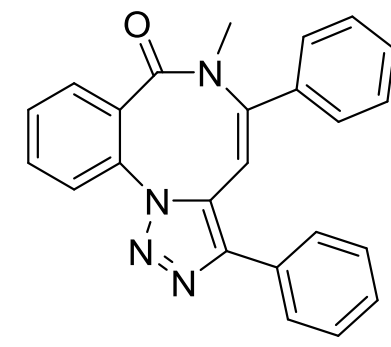

$3 a$

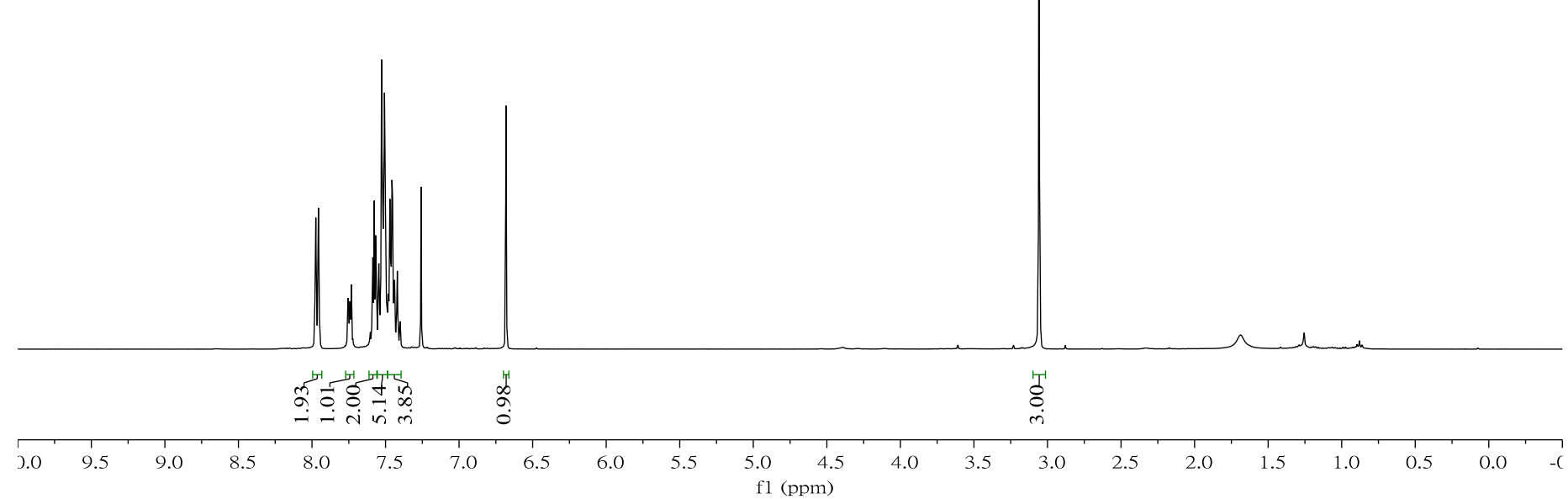

${ }^{1} \mathrm{H}$ NMR Spectrum (400 MHz) of compound 3a in $\mathrm{CDCl}_{3}$ 


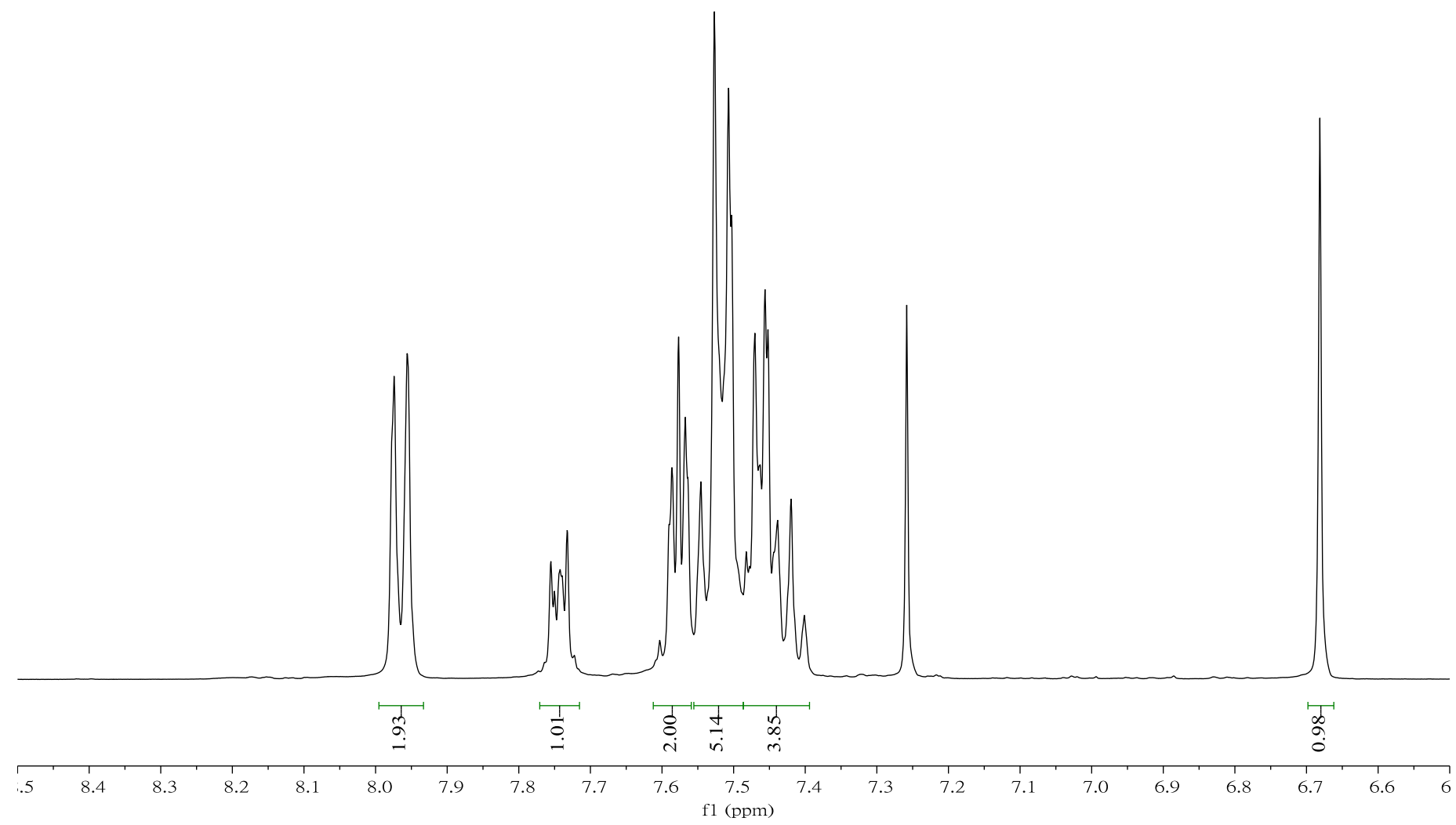

Expansion of ${ }^{1} \mathrm{H}$ NMR Spectrum (400 MHz) of compound 3a in $\mathrm{CDCl}_{3}$ 


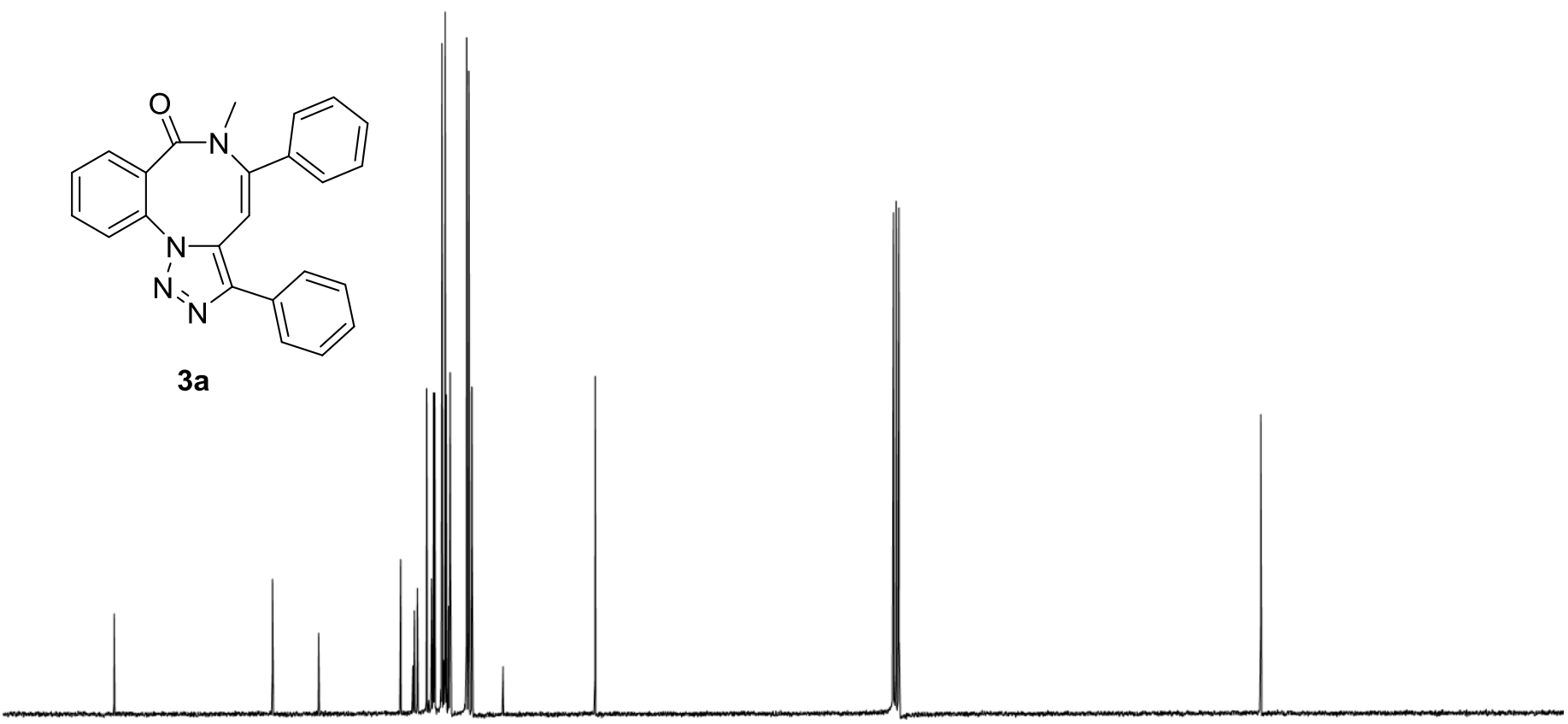

\section{0}

140 130 120 110

${ }^{13} \mathrm{C}$ NMR Spectrum (101 MHz) of compound $3 \mathbf{a}$ in $\mathrm{CDCl}_{3}$ 


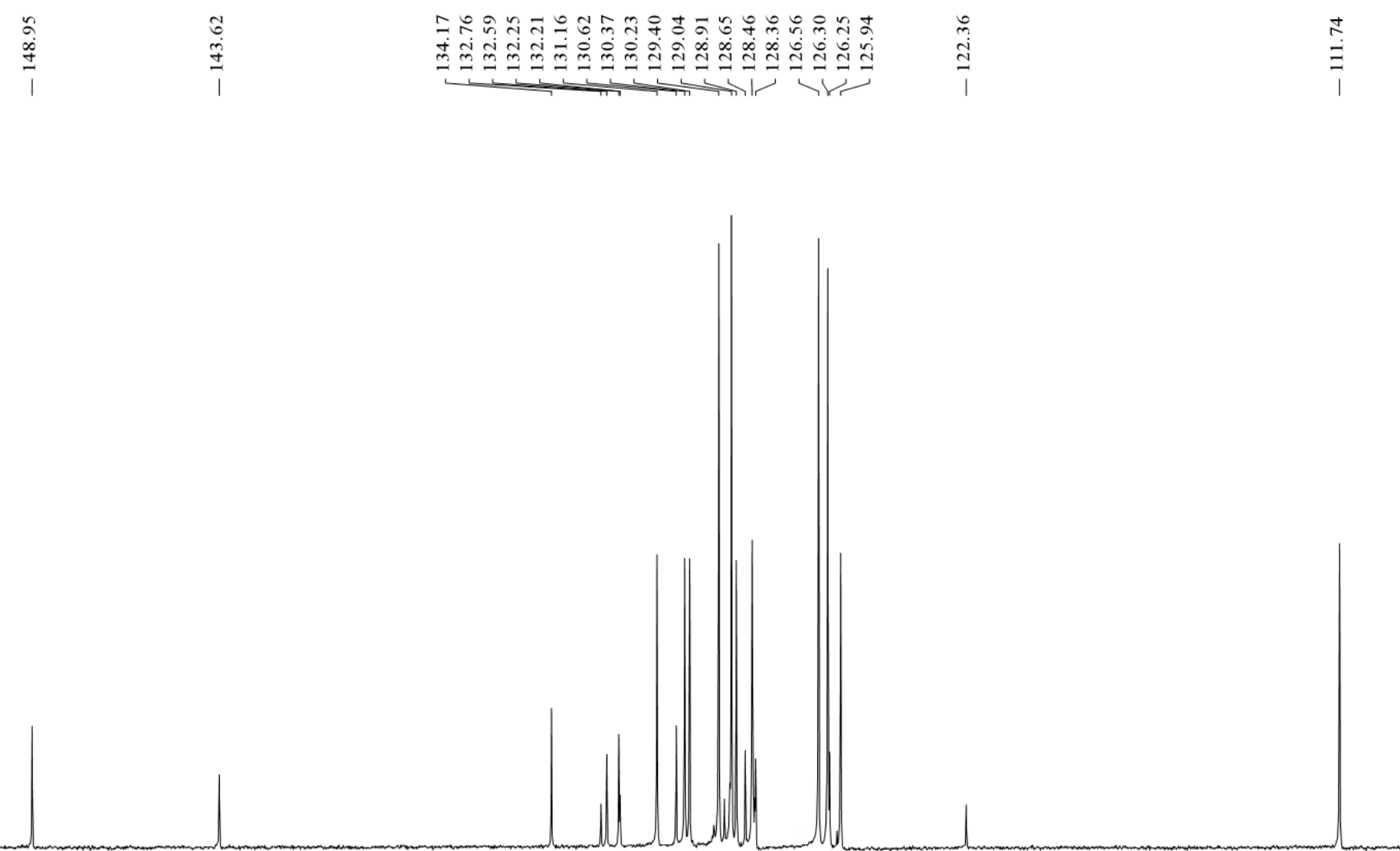

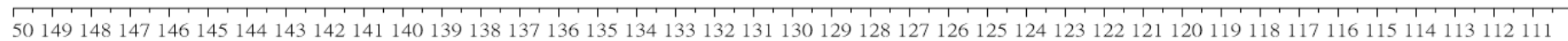
f1 (ppm)

Expansion of ${ }^{13} \mathrm{C}$ NMR Spectrum (101 MHz) of compound 3a in $\mathrm{CDCl}_{3}$ 
Display Report

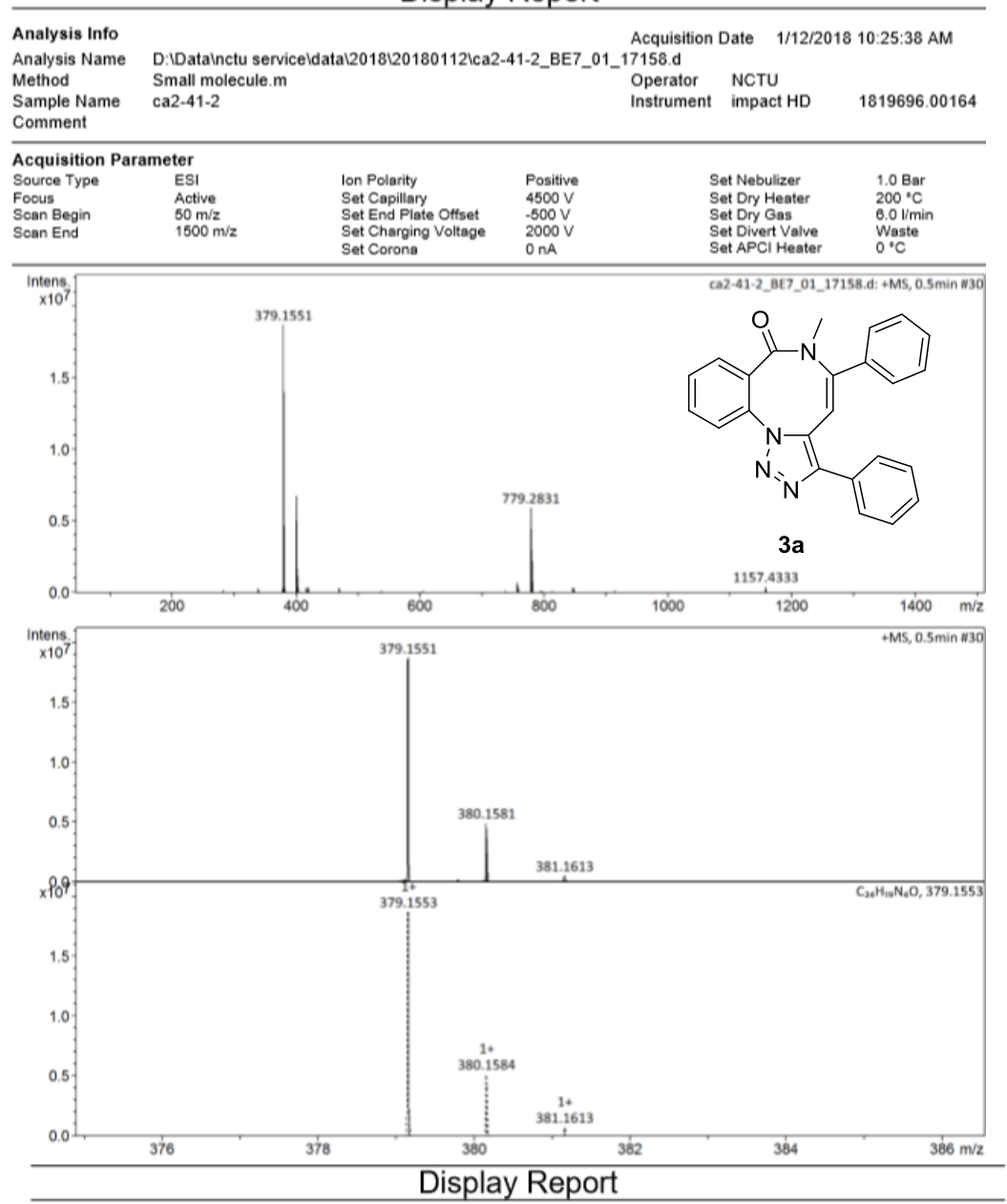

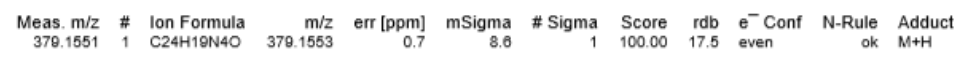

HRMS of compound $\mathbf{3 a}$ 


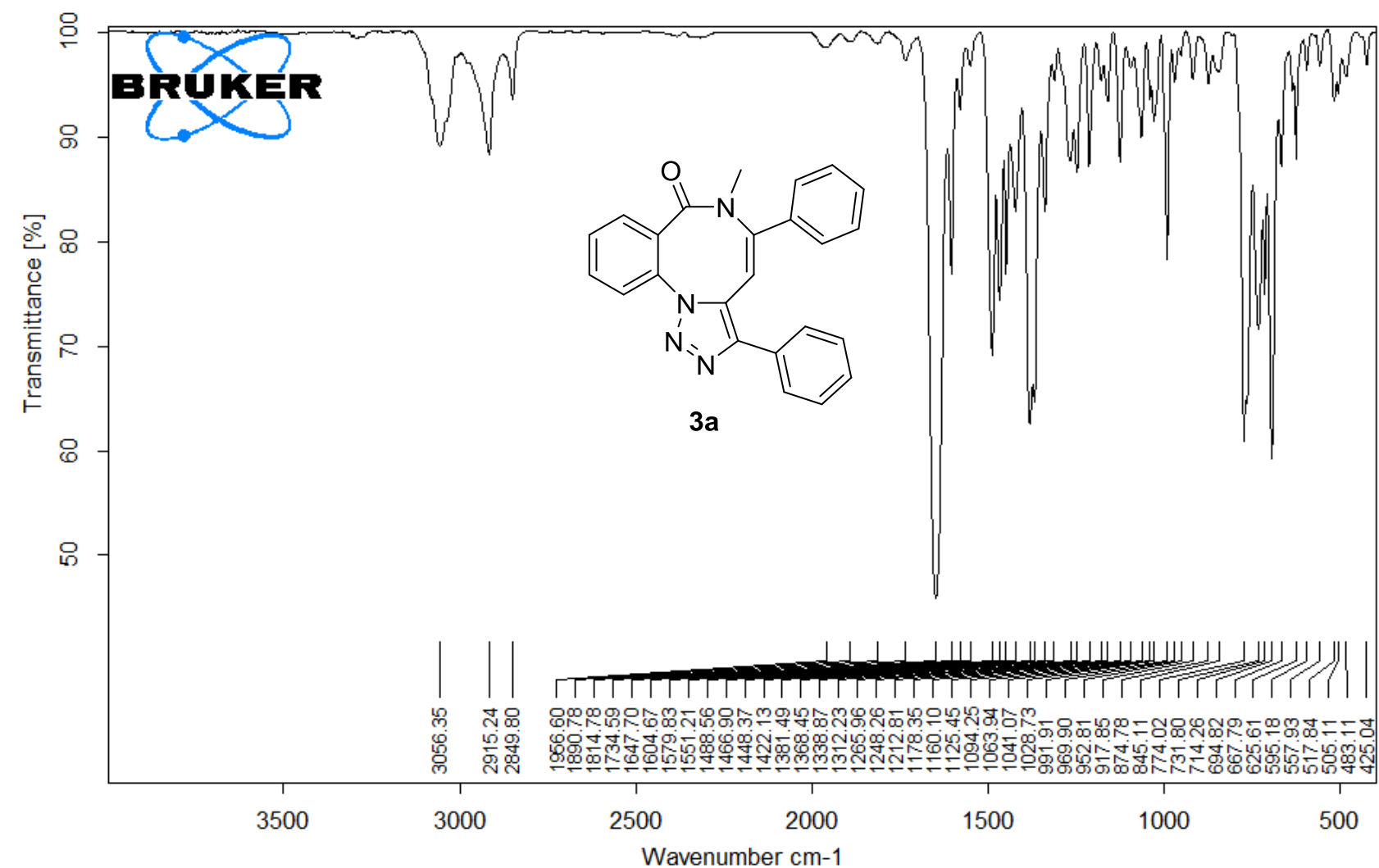

\begin{tabular}{|c|c|c|c|}
\hline 180821 & MIR_TR_DTGS_26128 & Instrument type and / or accessory & 8/21/2018 \\
\hline
\end{tabular}

Page 1/1

IR of compound $\mathbf{3 a}$ 


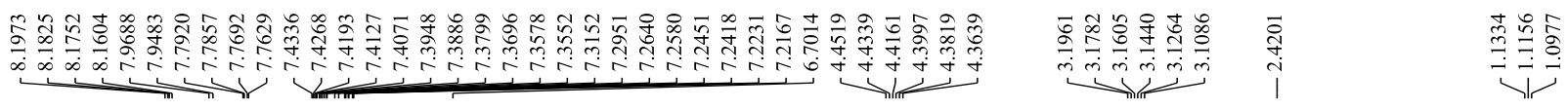

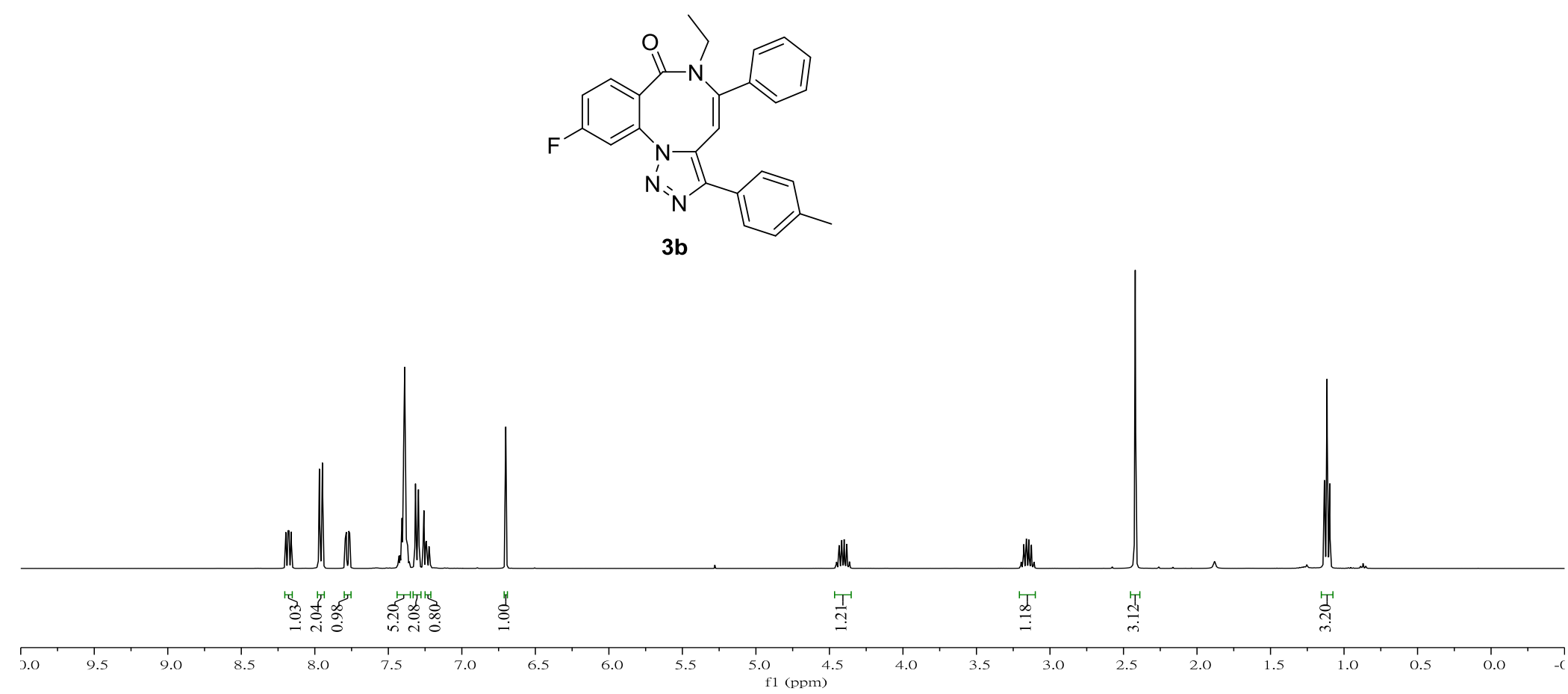

${ }^{1} \mathrm{H}$ NMR Spectrum (400 MHz) of compound $\mathbf{3 b}$ in $\mathrm{CDCl}_{3}$ 


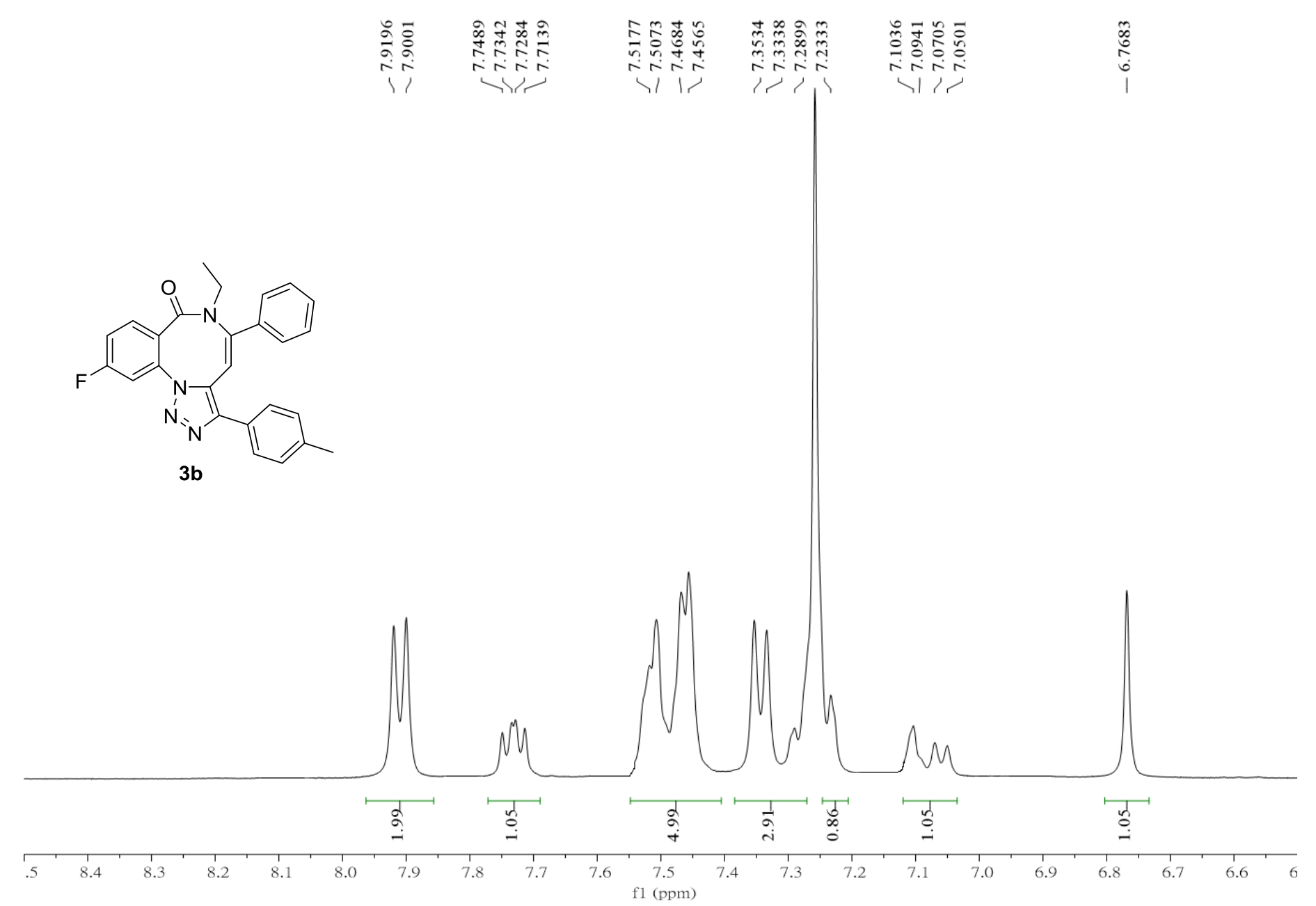

Expansion of ${ }^{1} \mathrm{H}$ NMR Spectrum (400 MHz) of compound $\mathbf{3 b}$ in $\mathrm{CDCl}_{3}$ 


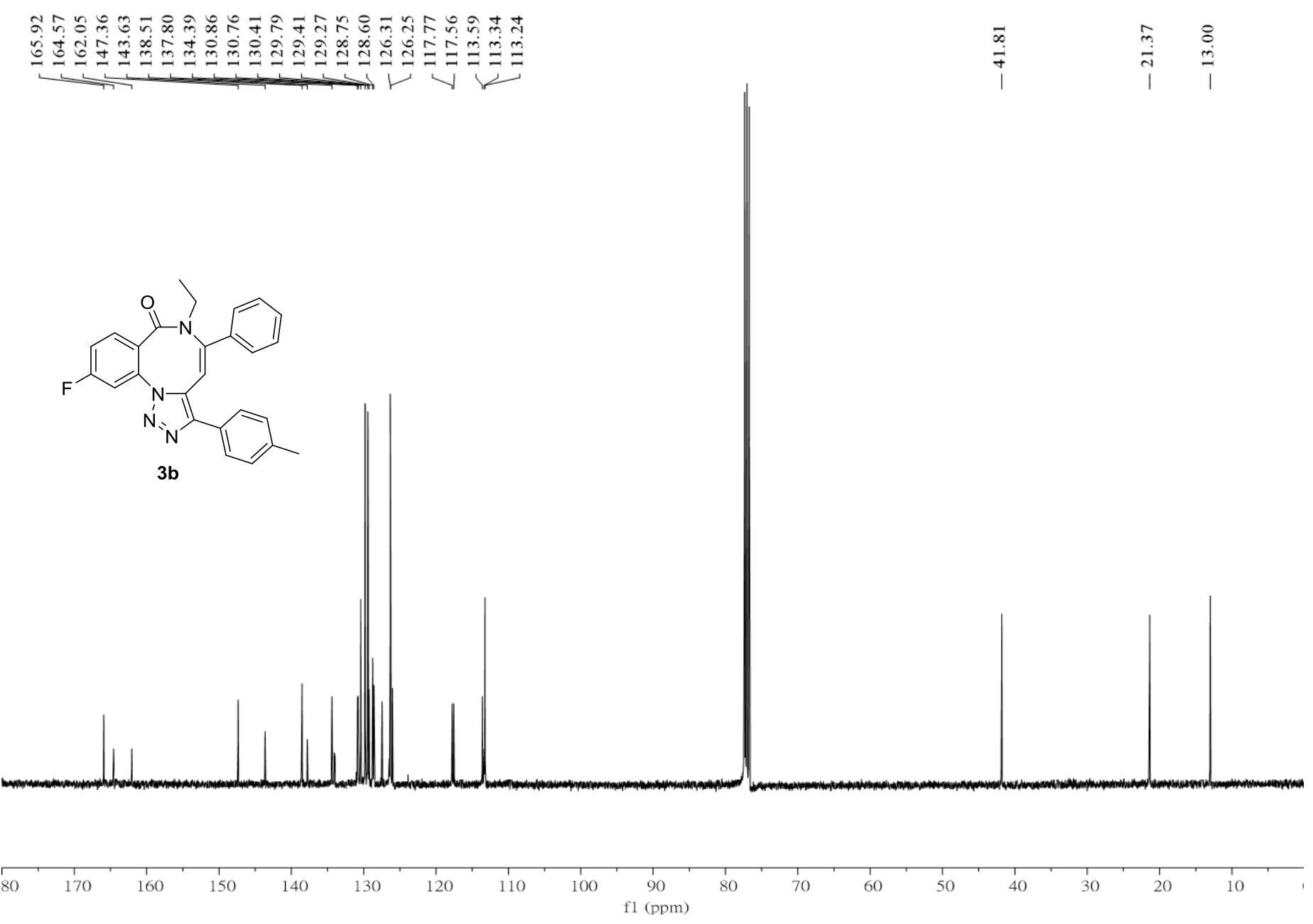

${ }^{13} \mathrm{C}$ NMR Spectrum (101 MHz) of compound $\mathbf{3 b}$ in $\mathrm{CDCl}_{3}$ 


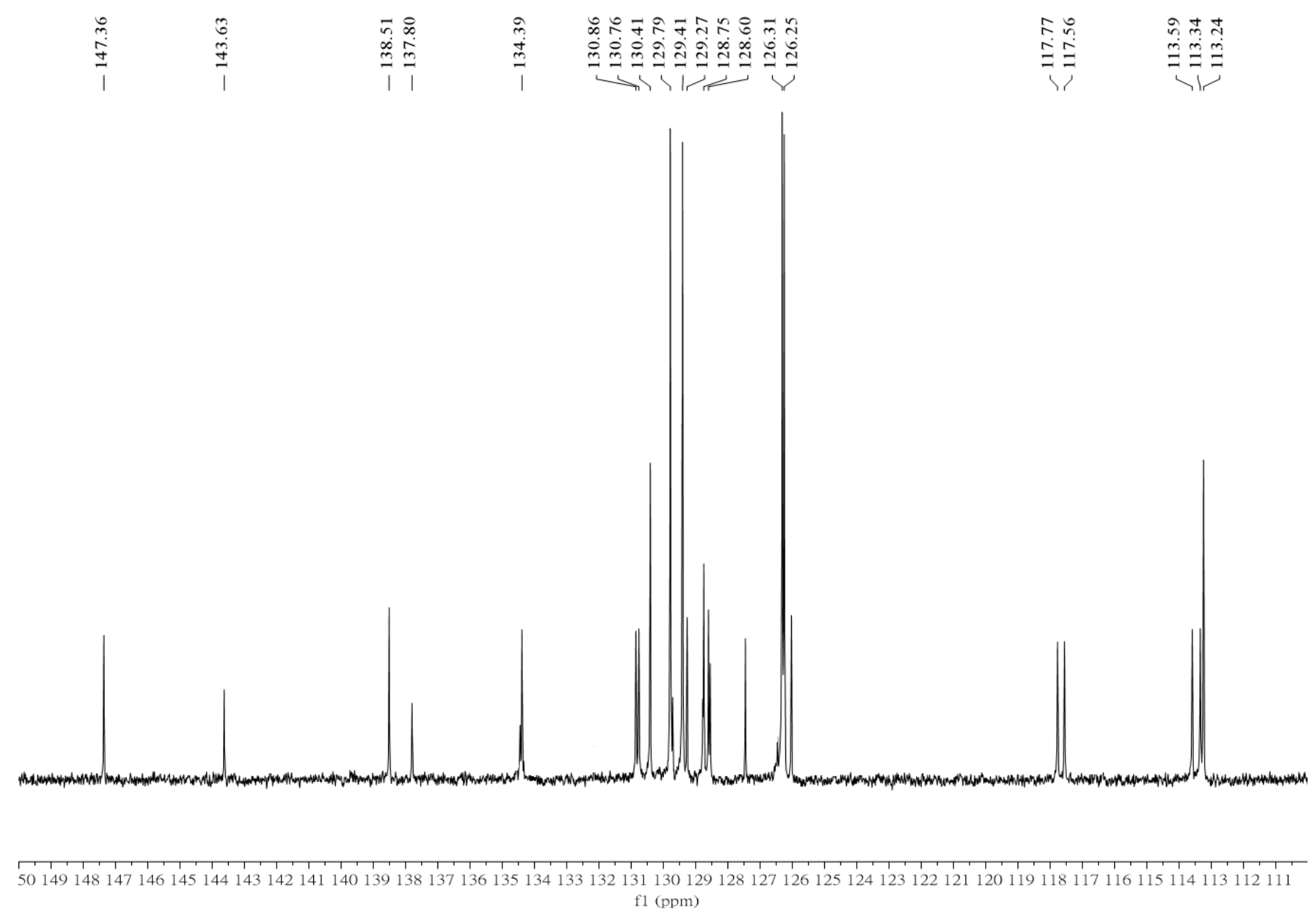

Expansion of ${ }^{13} \mathrm{C}$ NMR Spectrum (101 MHz) of compound $\mathbf{3 b}$ in $\mathrm{CDCl}_{3}$ 
Display Report

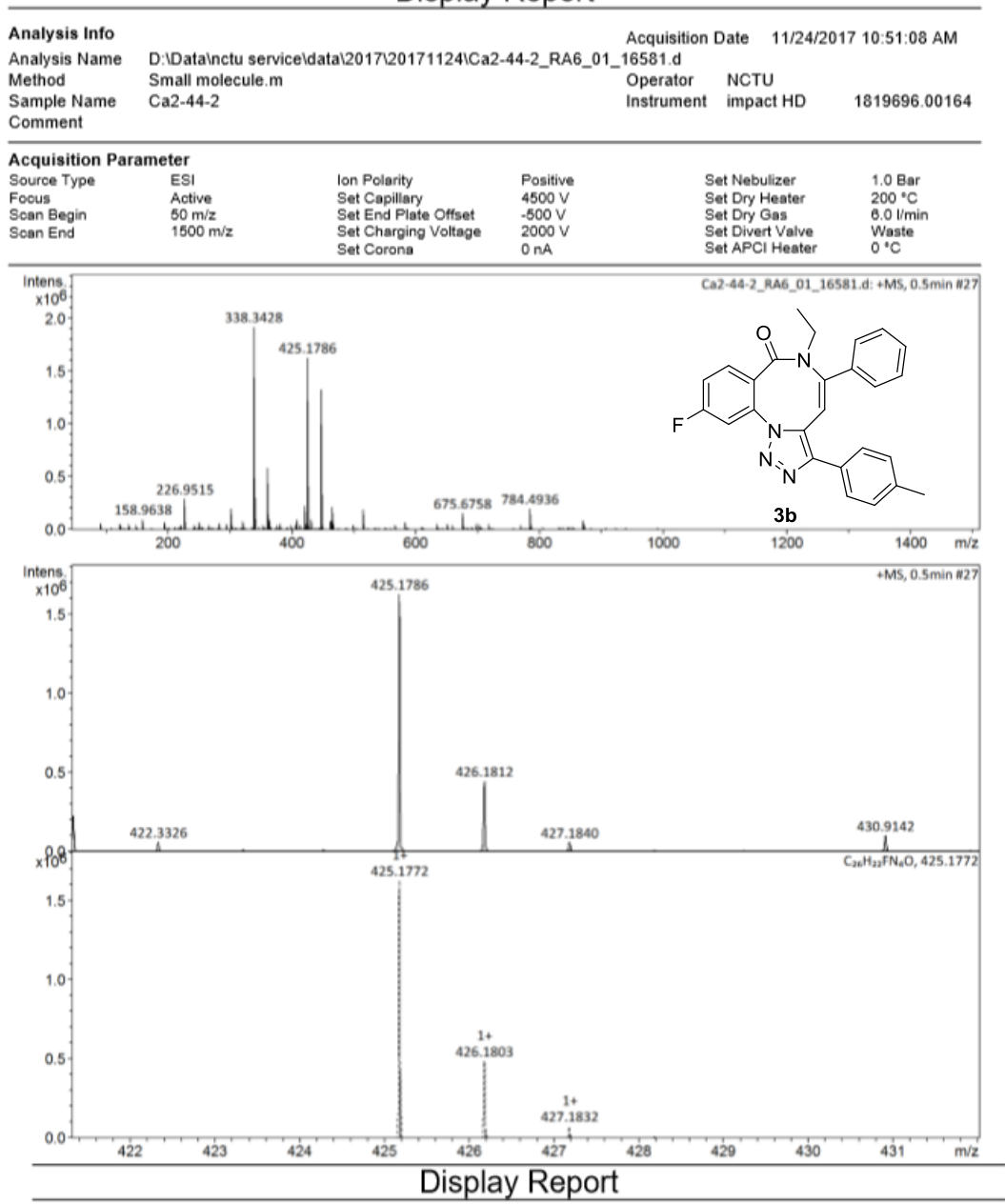

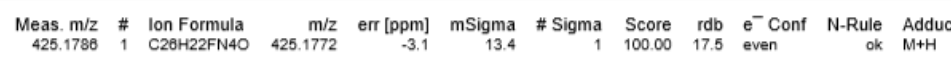

HRMS of compound $\mathbf{3 b}$ 


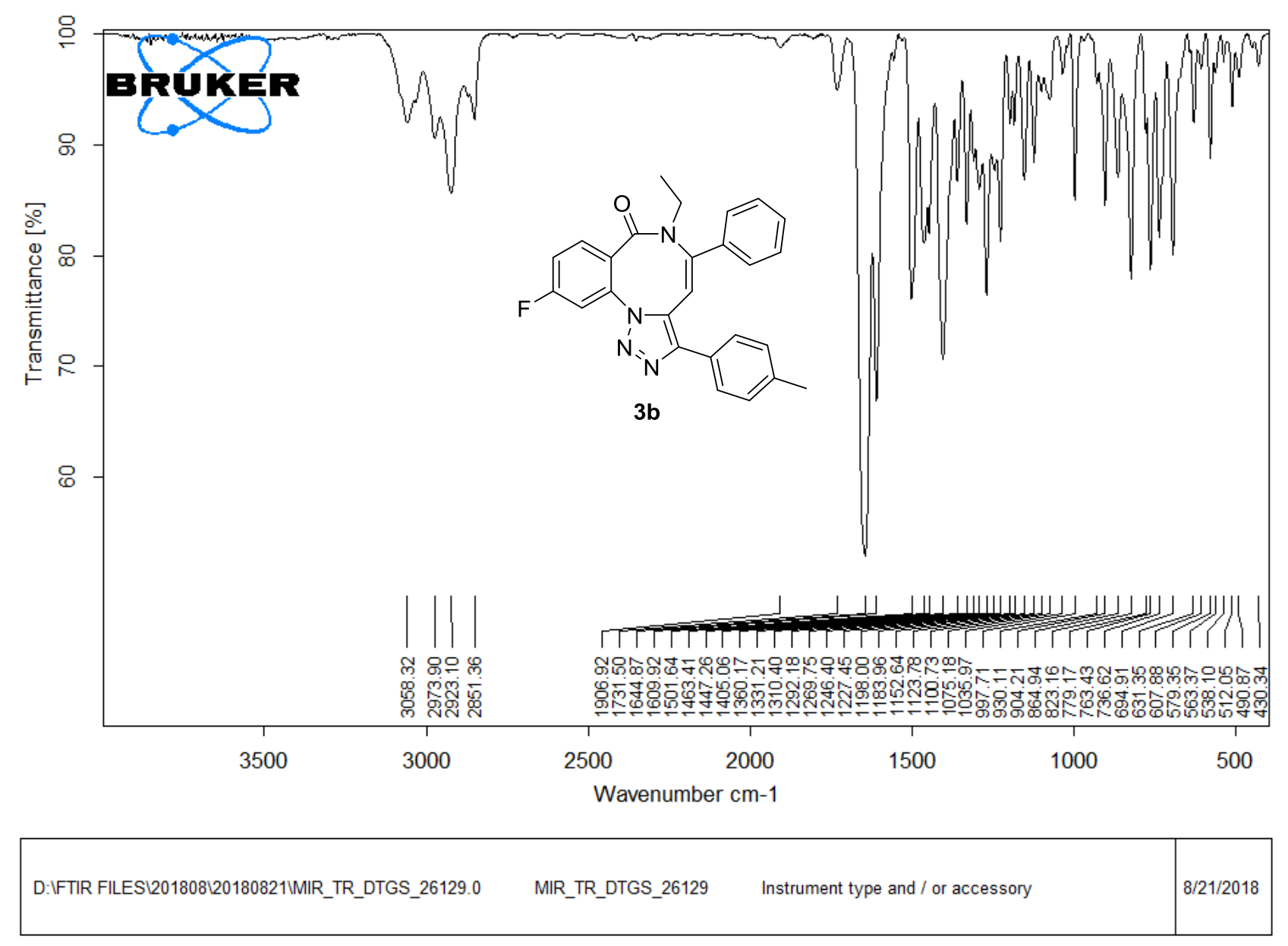

Page 1/1

\section{IR of compound $\mathbf{3 b}$}




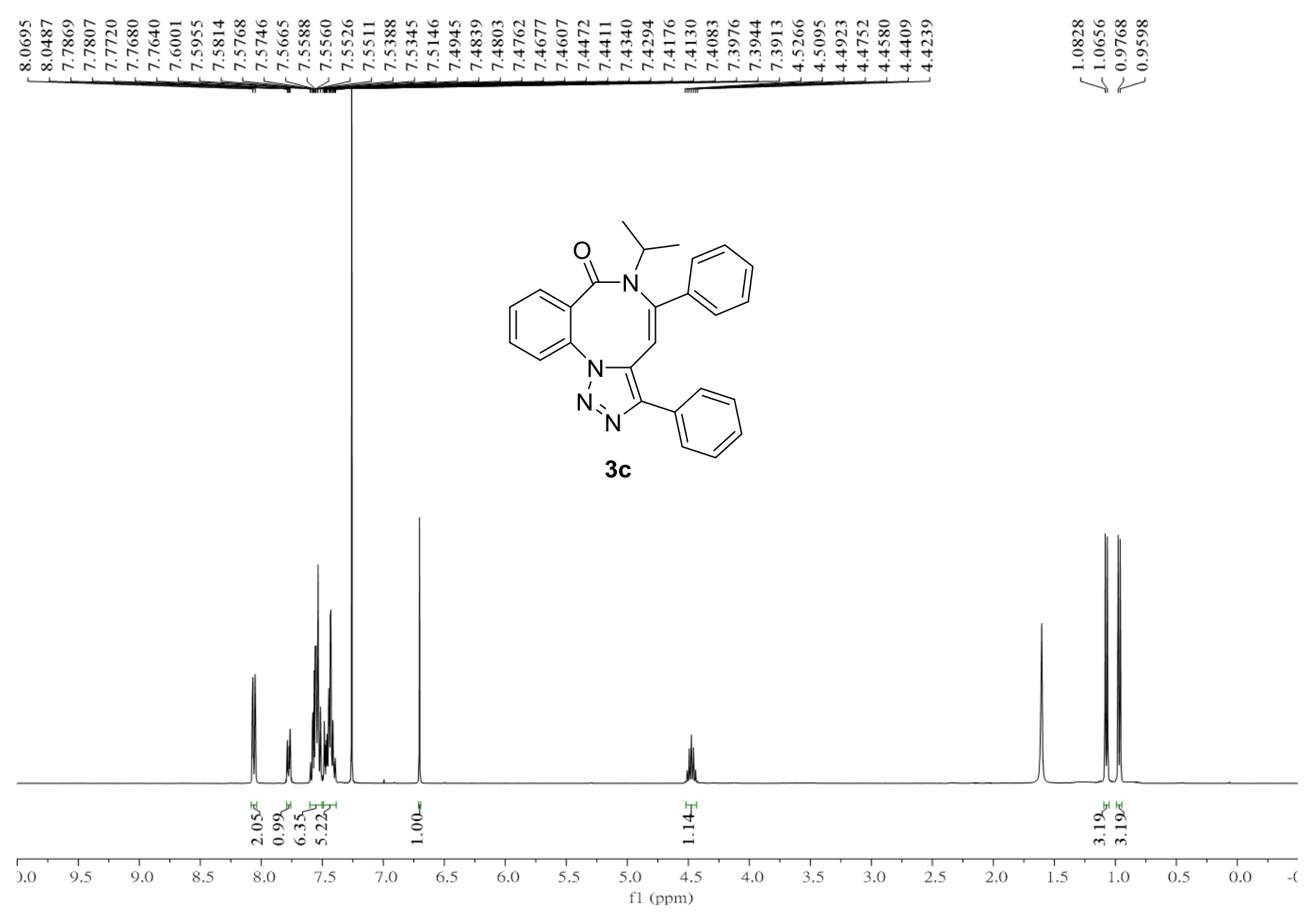

${ }^{1} \mathrm{H}$ NMR Spectrum (400 MHz) of compound $\mathbf{3 c}$ in $\mathrm{CDCl}_{3}$ 


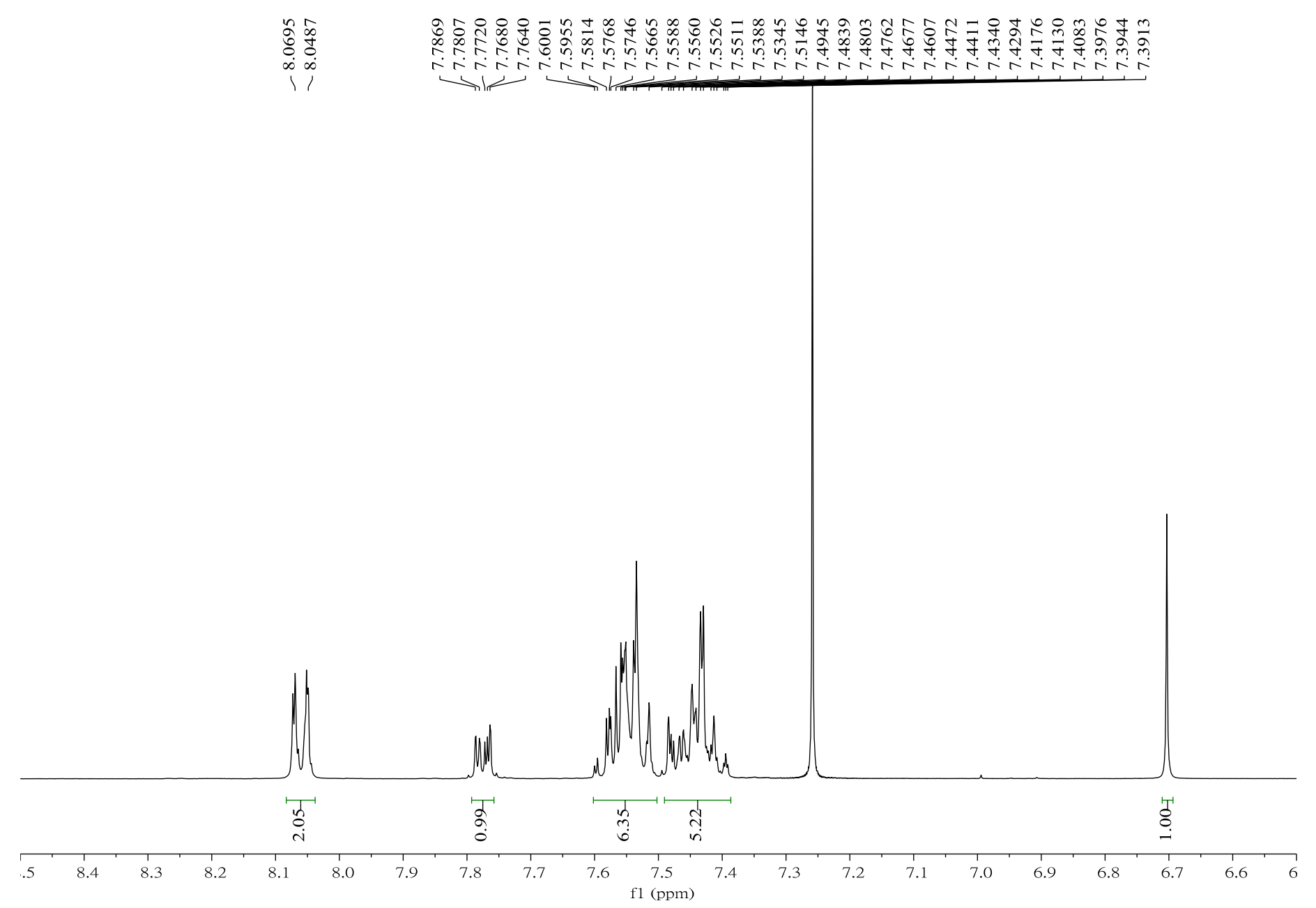

Expansion of ${ }^{1} \mathrm{H}$ NMR Spectrum (400 MHz) of compound $3 \mathbf{c}$ in $\mathrm{CDCl}_{3}$ 


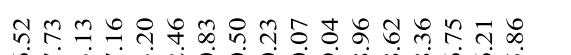

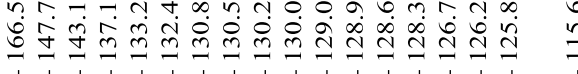

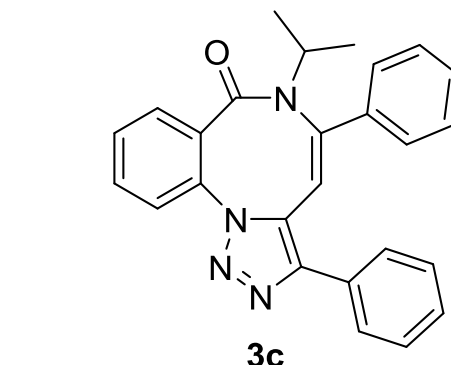

$3 c$
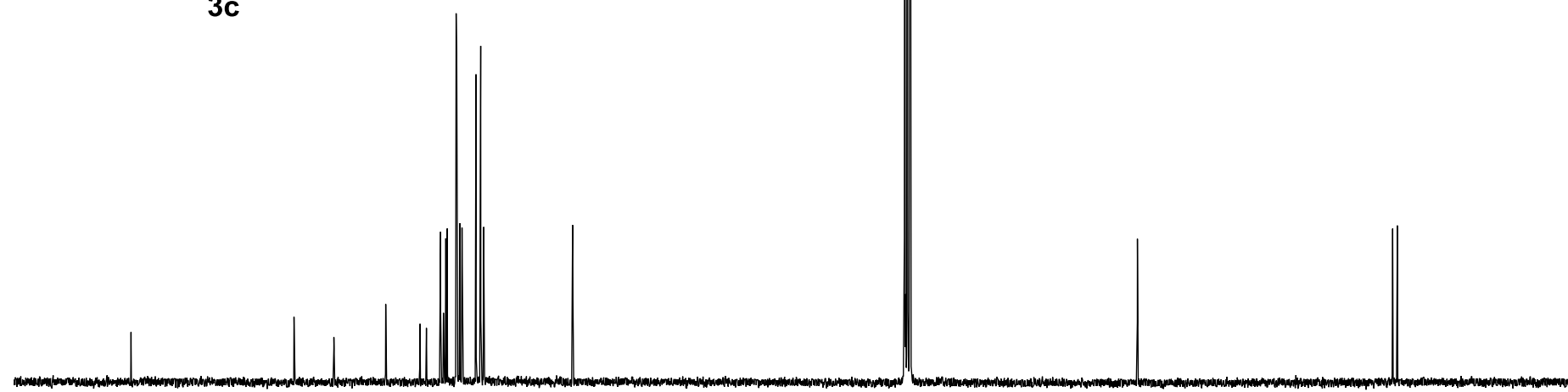

80

170

$160 \quad 150$

140

$130 \quad 120$

110

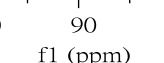

$80 \quad 70$

60

40

${ }^{13} \mathrm{C}$ NMR Spectrum $\left(101 \mathrm{MHz}\right.$ ) of compound $\mathbf{3 c}$ in $\mathrm{CDCl}_{3}$ 


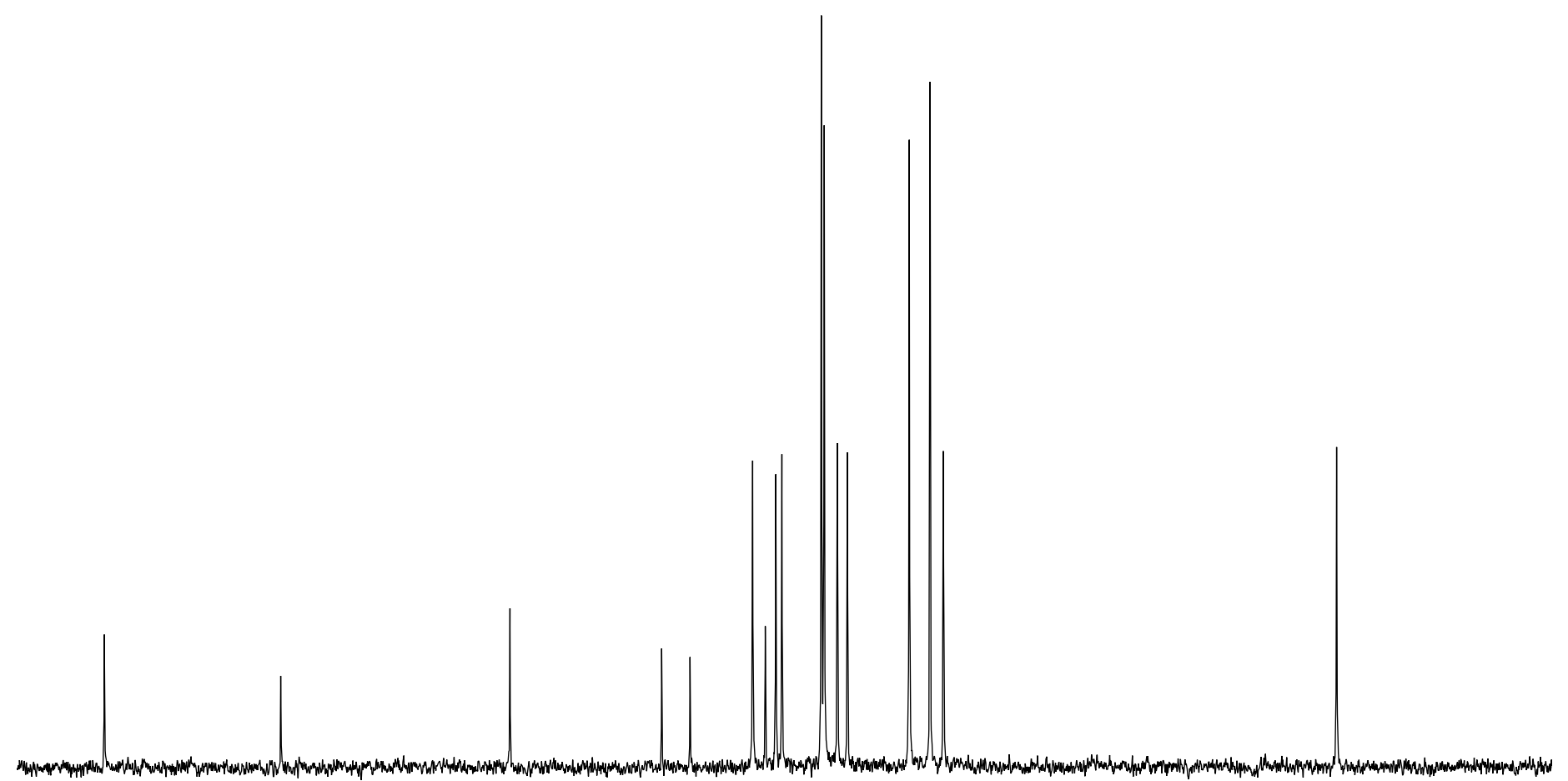

50149148147146145144143142141140139138137136135134133132131130129128127126125124123122121120119118117116115114113112111 f1 (ppm)

Expansion of ${ }^{13} \mathrm{C}$ NMR Spectrum (101 MHz) of compound $\mathbf{3 c}$ in $\mathrm{CDCl}_{3}$ 
Display Report

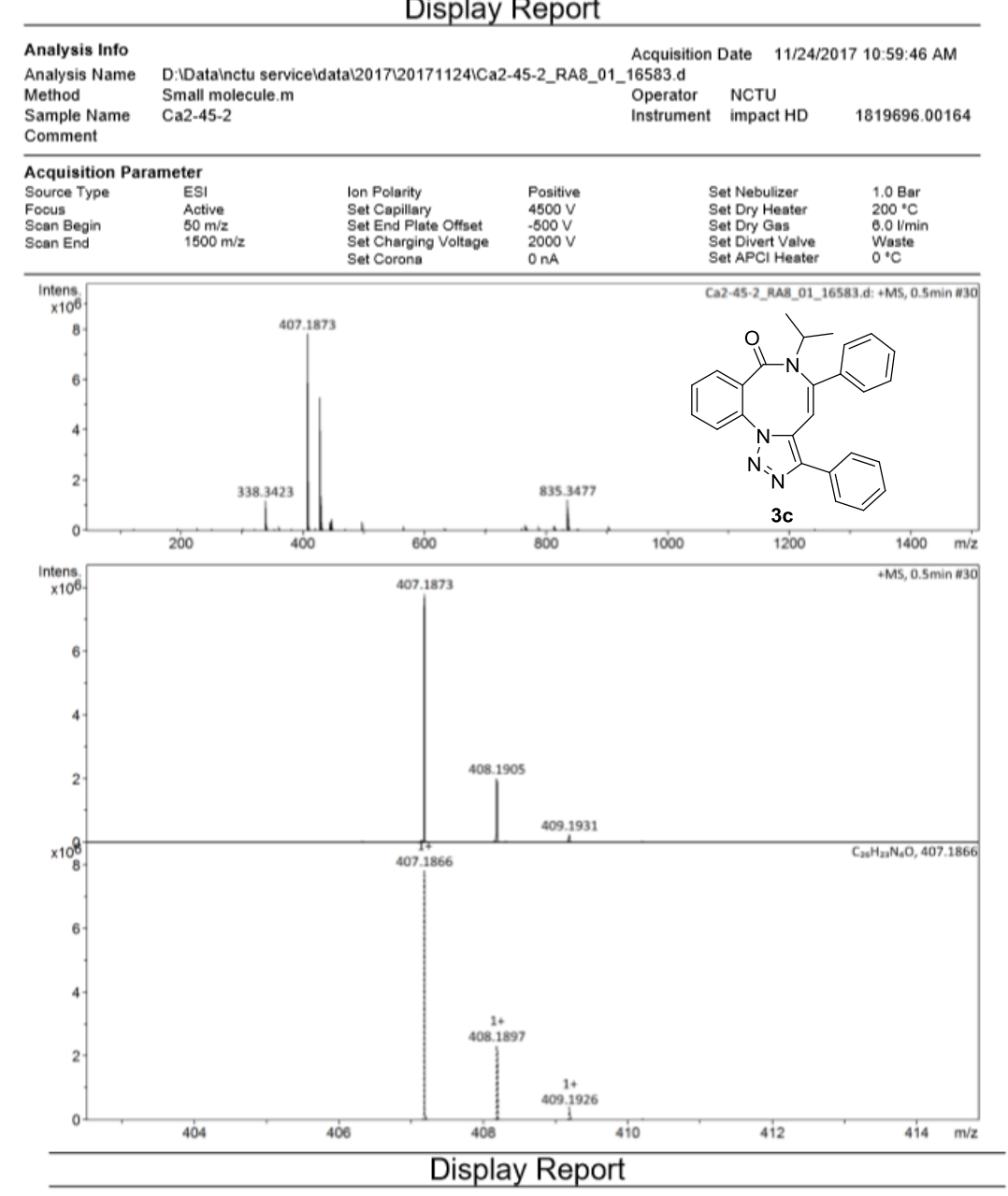

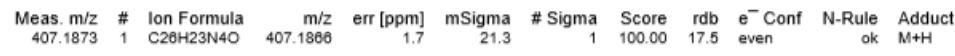

HRMS of compound $\mathbf{3 c}$ 


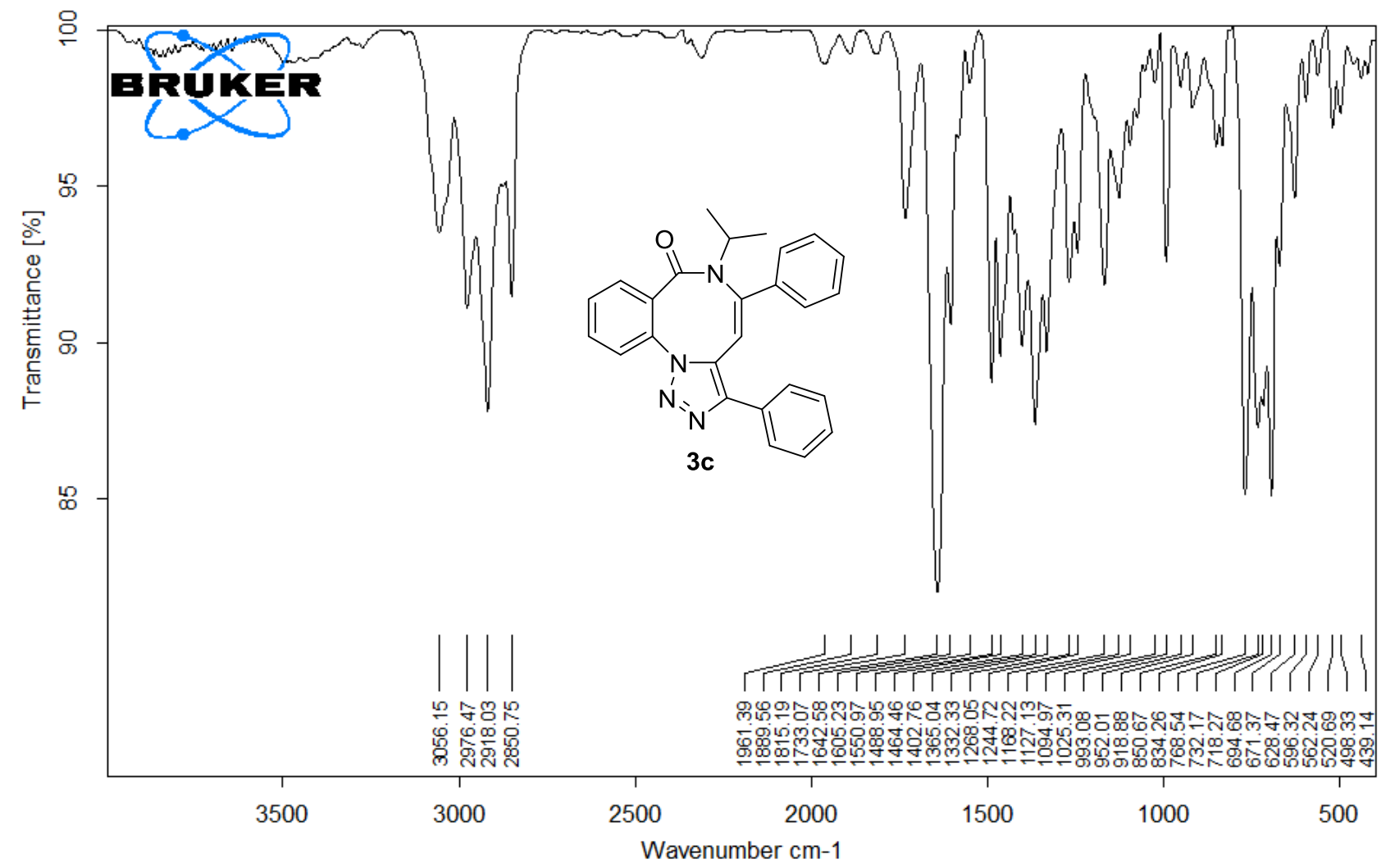

\begin{tabular}{|lcl|l|}
\hline D:IFTIR FILESL201808120180821MMIR_TR_DTGS_26130.0 MIR_TR_DTGS_26130 Instrument type and / or accessory & $8 / 21 / 2018$ \\
\hline
\end{tabular}

Page 1/1

IR of compound $\mathbf{3 c}$ 


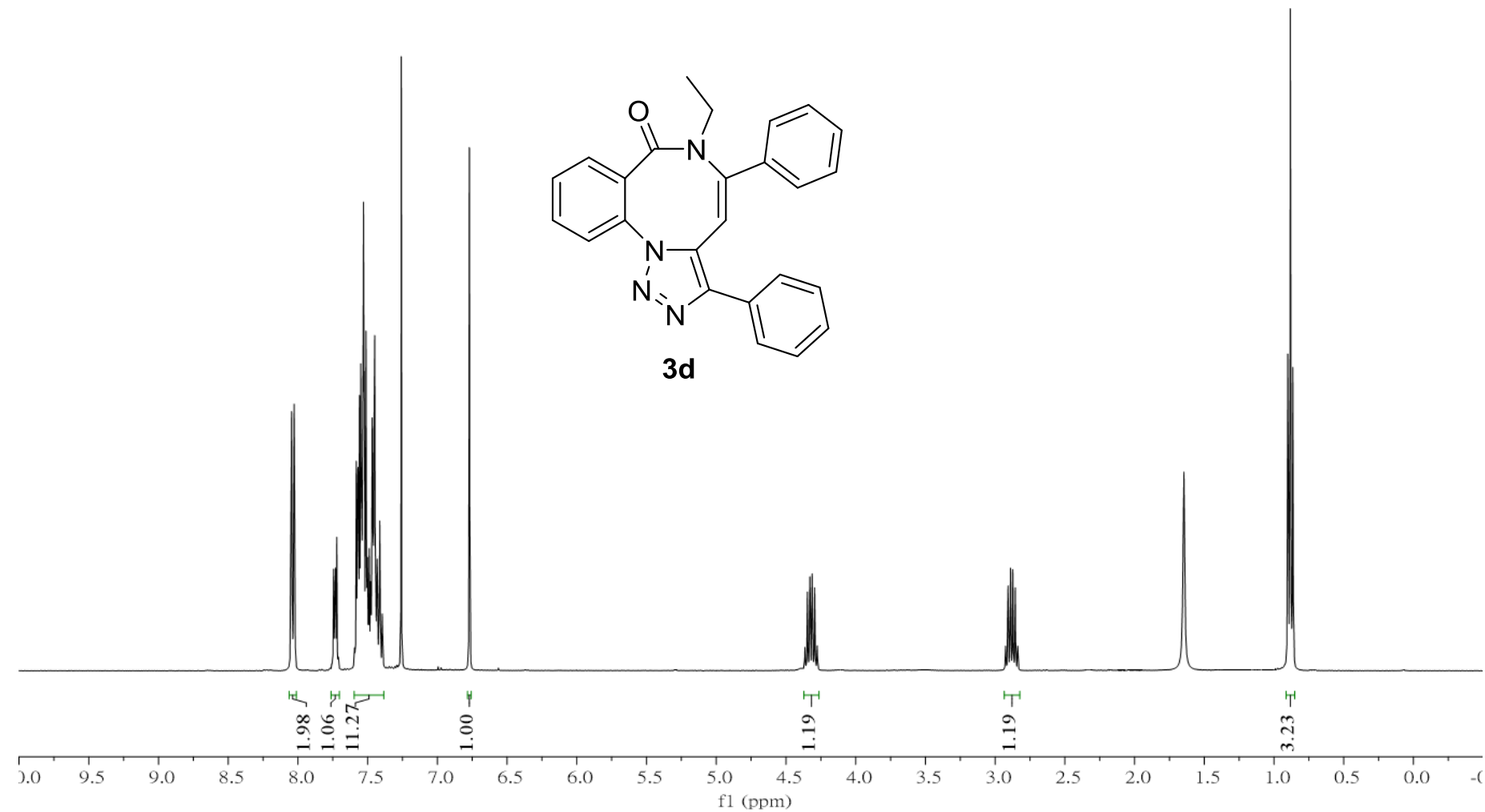

${ }^{1} \mathrm{H}$ NMR Spectrum $\left(400 \mathrm{MHz}\right.$ ) of compound $\mathbf{3 d}$ in $\mathrm{CDCl}_{3}$ 


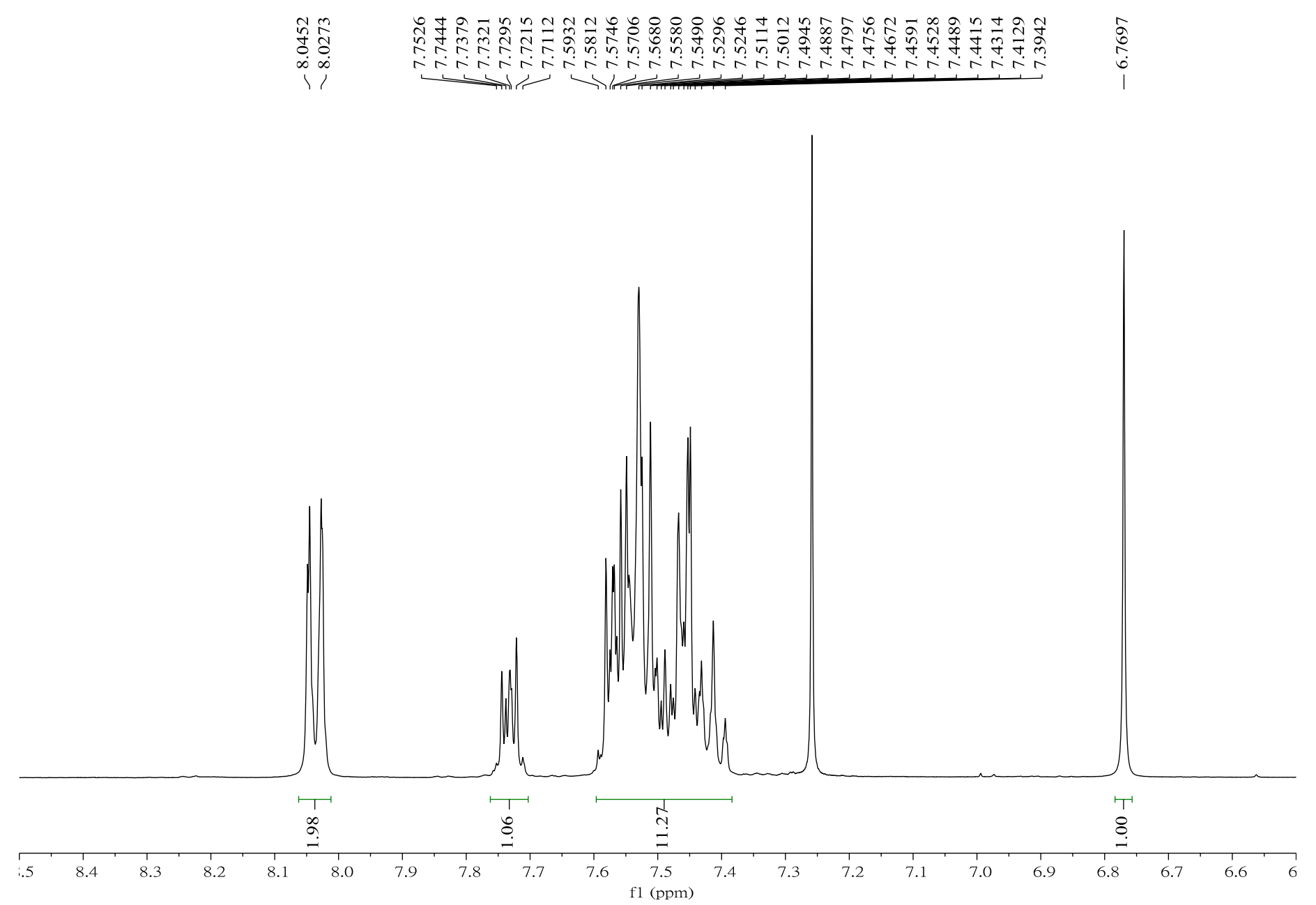

Expansion of ${ }^{1} \mathrm{H}$ NMR Spectrum (400 MHz) of compound 3d in $\mathrm{CDCl}_{3}$ 
ঢ.

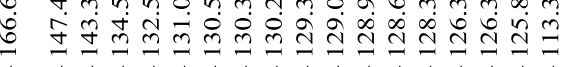

$1+1$

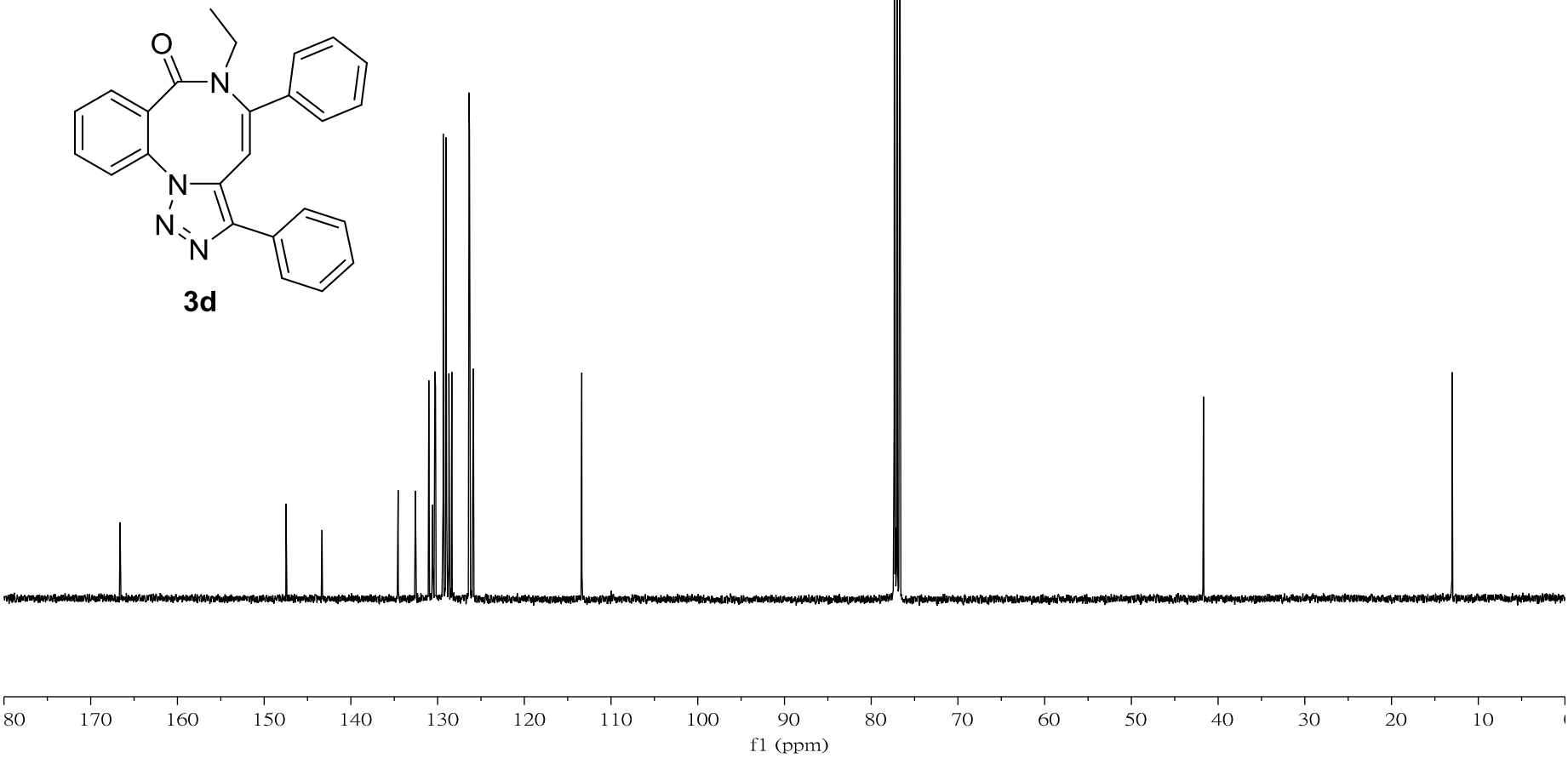

${ }^{13} \mathrm{C}$ NMR Spectrum (101 MHz) of compound $\mathbf{3 d}$ in $\mathrm{CDCl}_{3}$ 


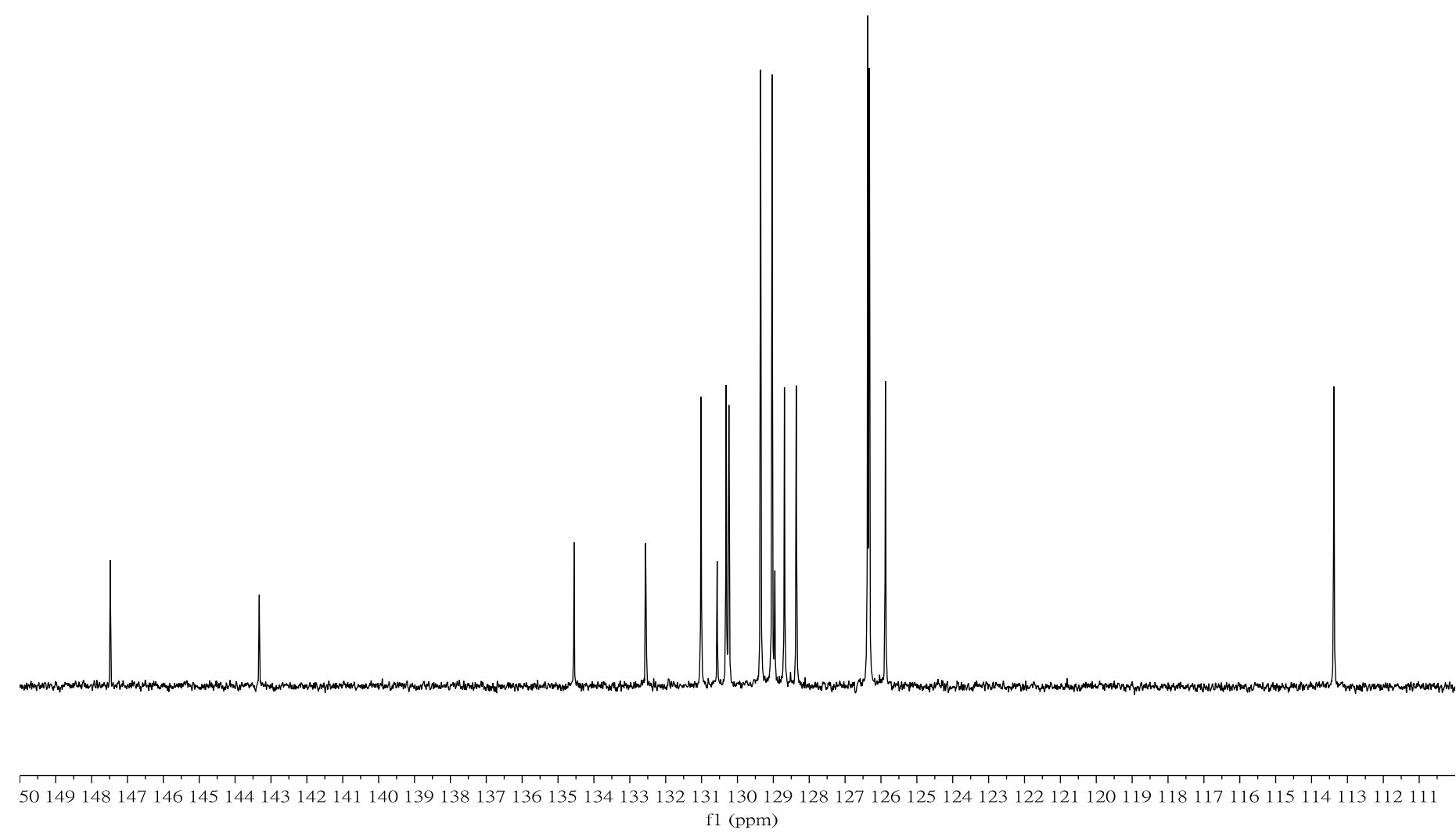

Expansion of ${ }^{13} \mathrm{C}$ NMR Spectrum (101 MHz) of compound 3d in $\mathrm{CDCl}_{3}$ 
Display Report

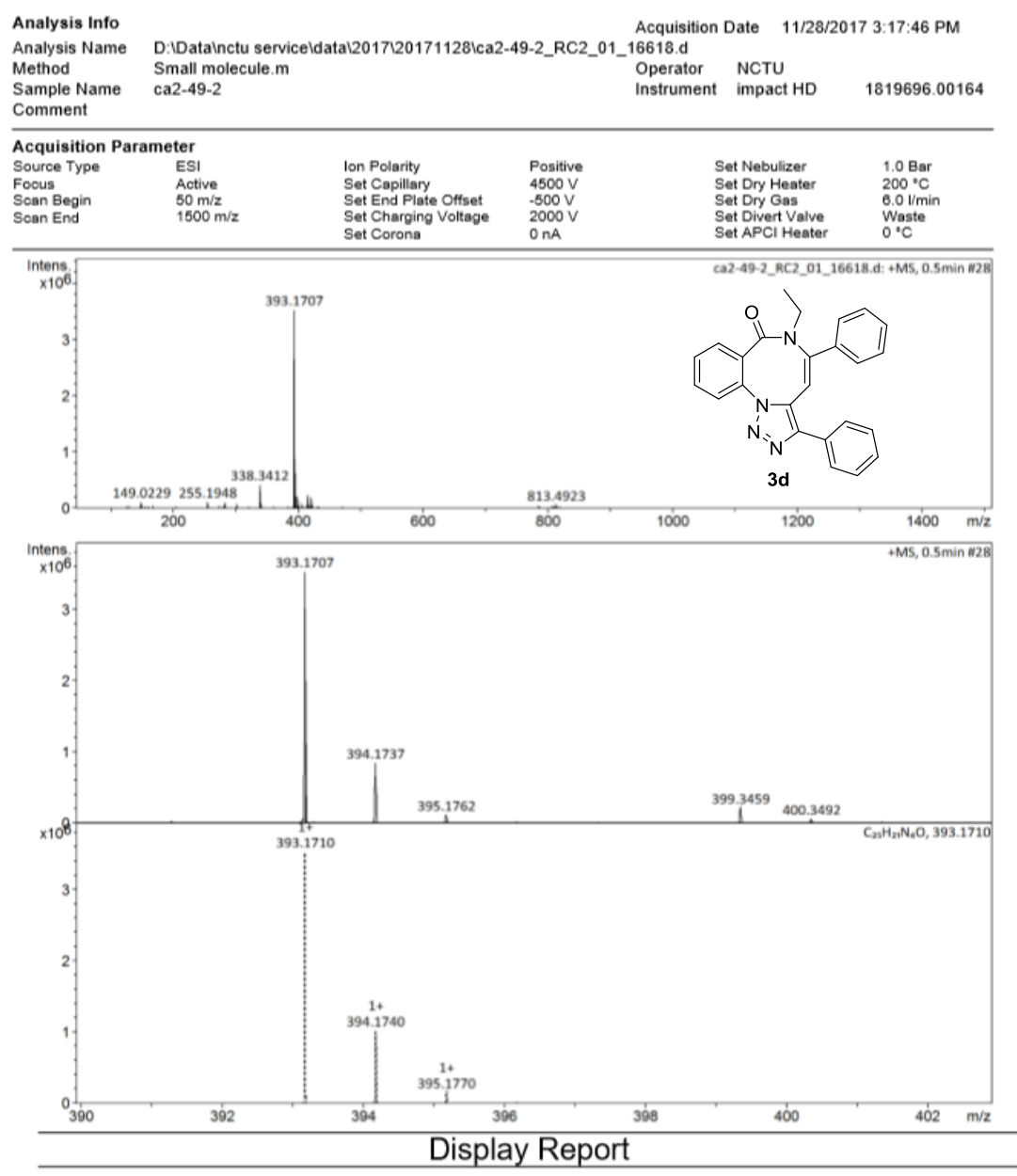

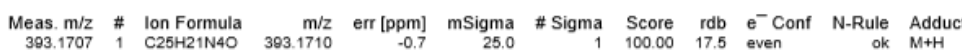

HRMS of compound $\mathbf{3 d}$ 


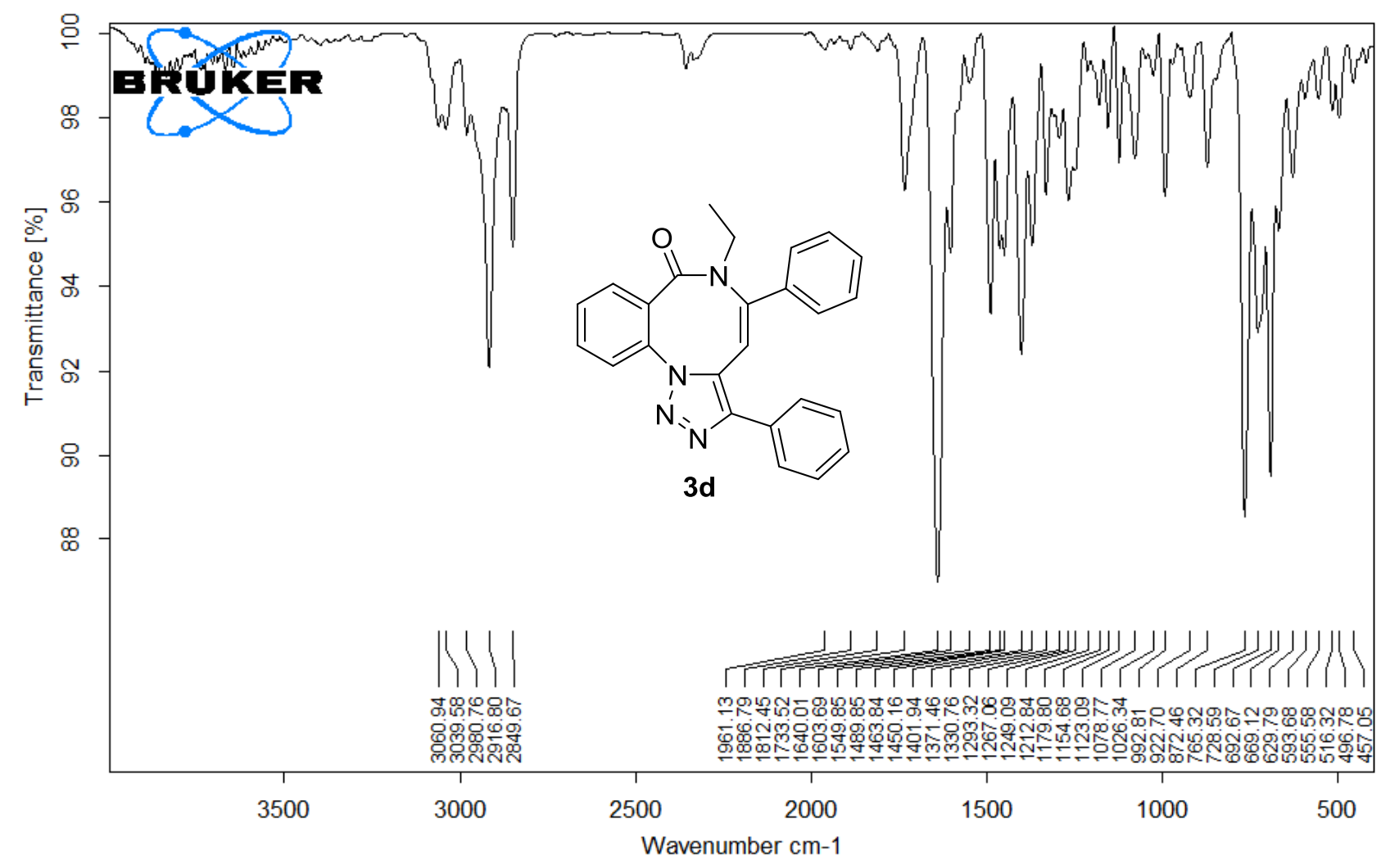

\begin{tabular}{|lcl|l|l|}
\hline D:IFTIR FILESI201808120180821IMIR_TR_DTGS_26131.0 $\quad$ MIR_TR_DTGS_26131 $\quad$ Instrument type and / or accessory & $8 / 21 / 2018$ \\
\hline
\end{tabular}

Page 1/1

\section{IR of compound $\mathbf{3 d}$}



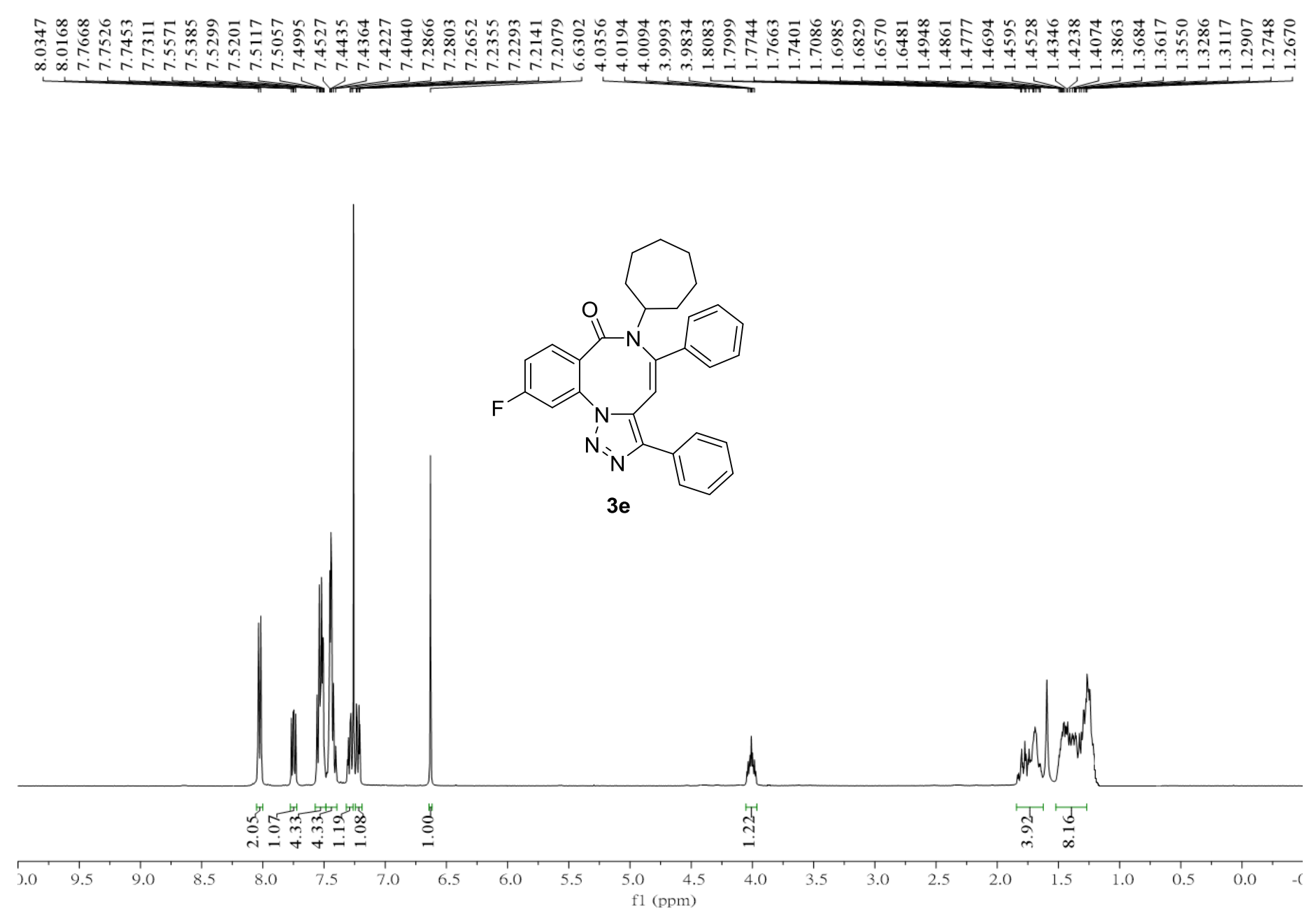

${ }^{1} \mathrm{H}$ NMR Spectrum $\left(400 \mathrm{MHz}\right.$ ) of compound $3 \mathbf{e}$ in $\mathrm{CDCl}_{3}$ 


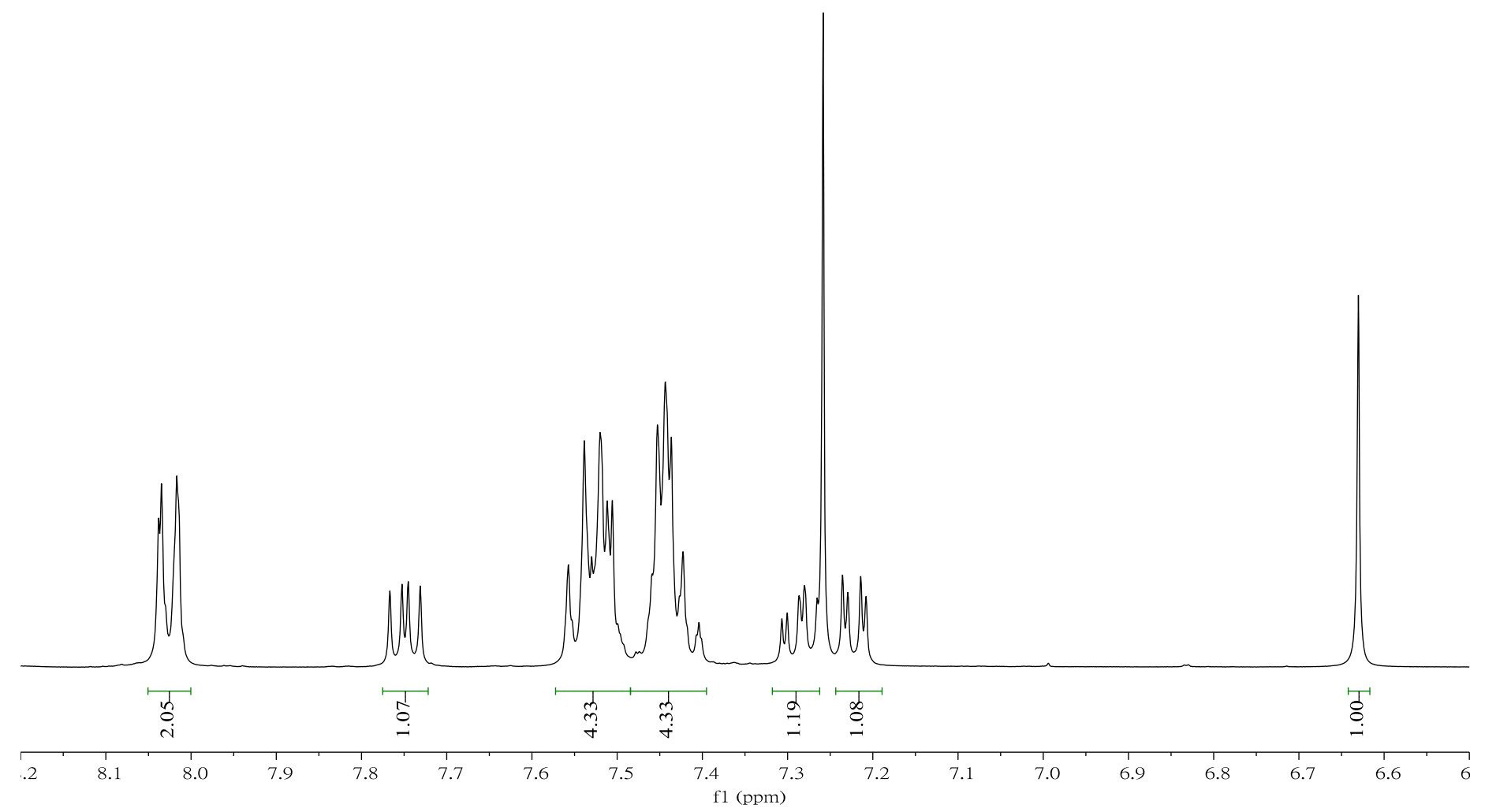

Expansion of ${ }^{1} \mathrm{H}$ NMR Spectrum (400 MHz) of compound 3e in $\mathrm{CDCl}_{3}$ 


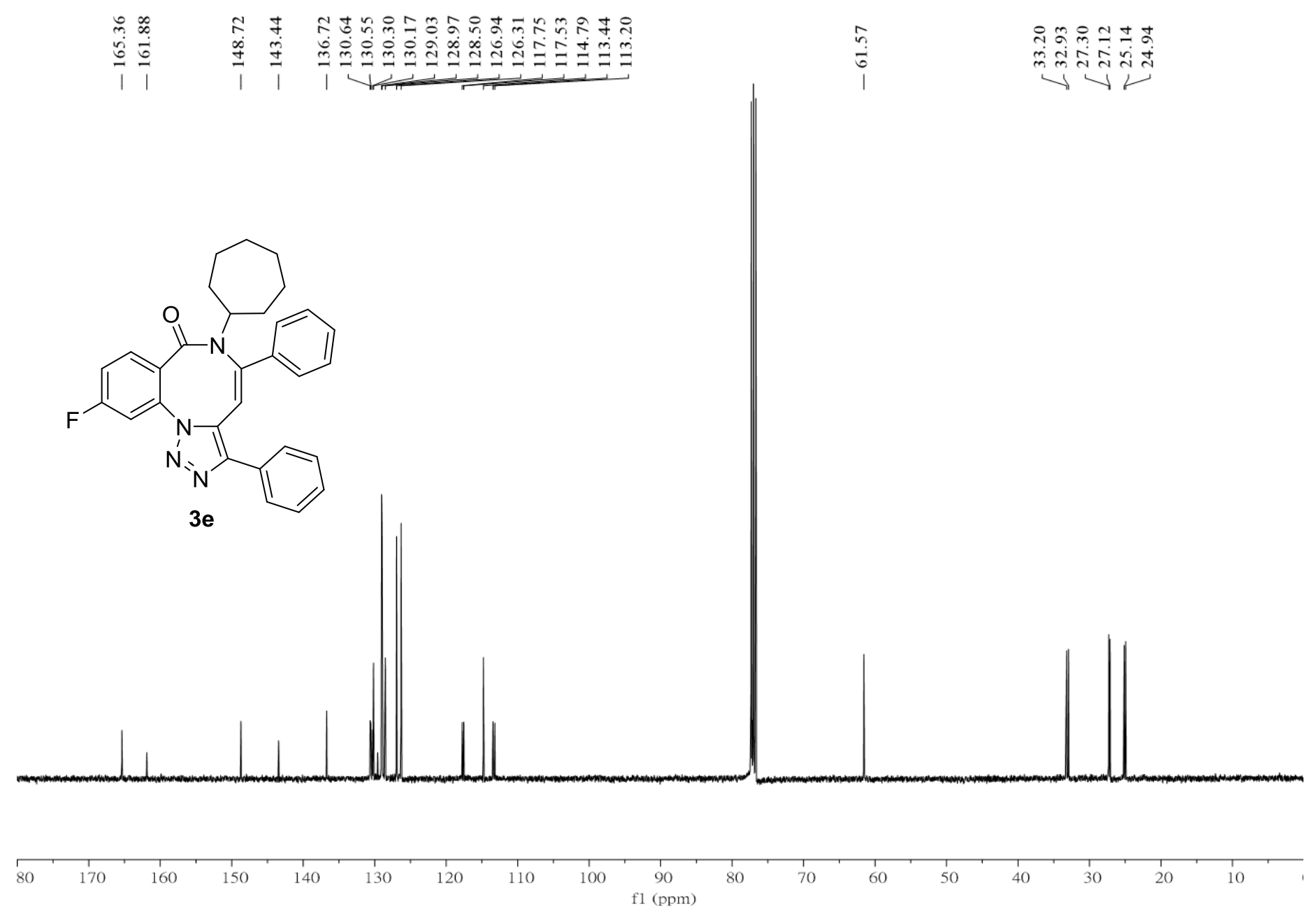

${ }^{13} \mathrm{C}$ NMR Spectrum (101 MHz) of compound $\mathbf{3 e}$ in $\mathrm{CDCl}_{3}$ 


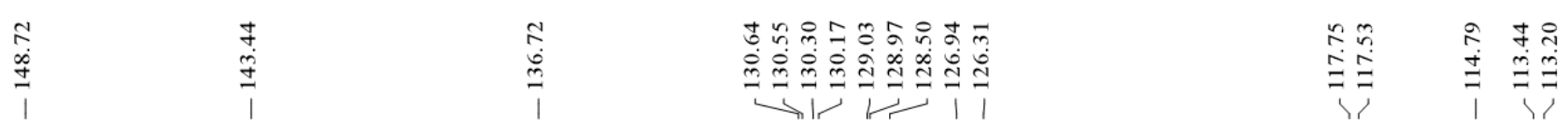

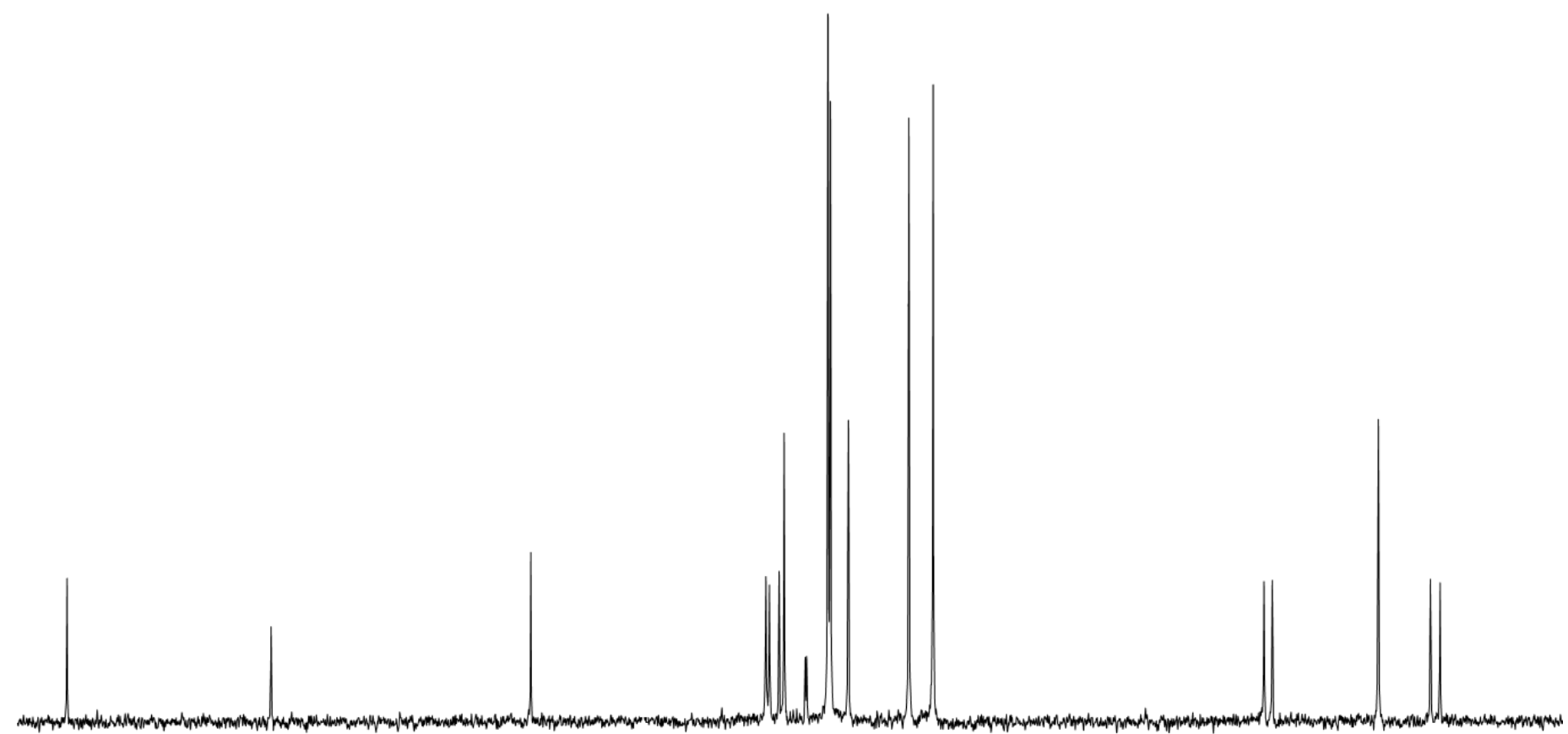

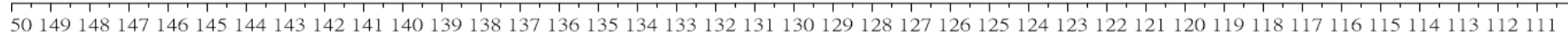
$\mathrm{fl}(\mathrm{ppm})$

Expansion of ${ }^{13} \mathrm{C}$ NMR Spectrum $(101 \mathrm{MHz})$ of compound $\mathbf{3 e}$ in $\mathrm{CDCl}_{3}$ 
Display Report

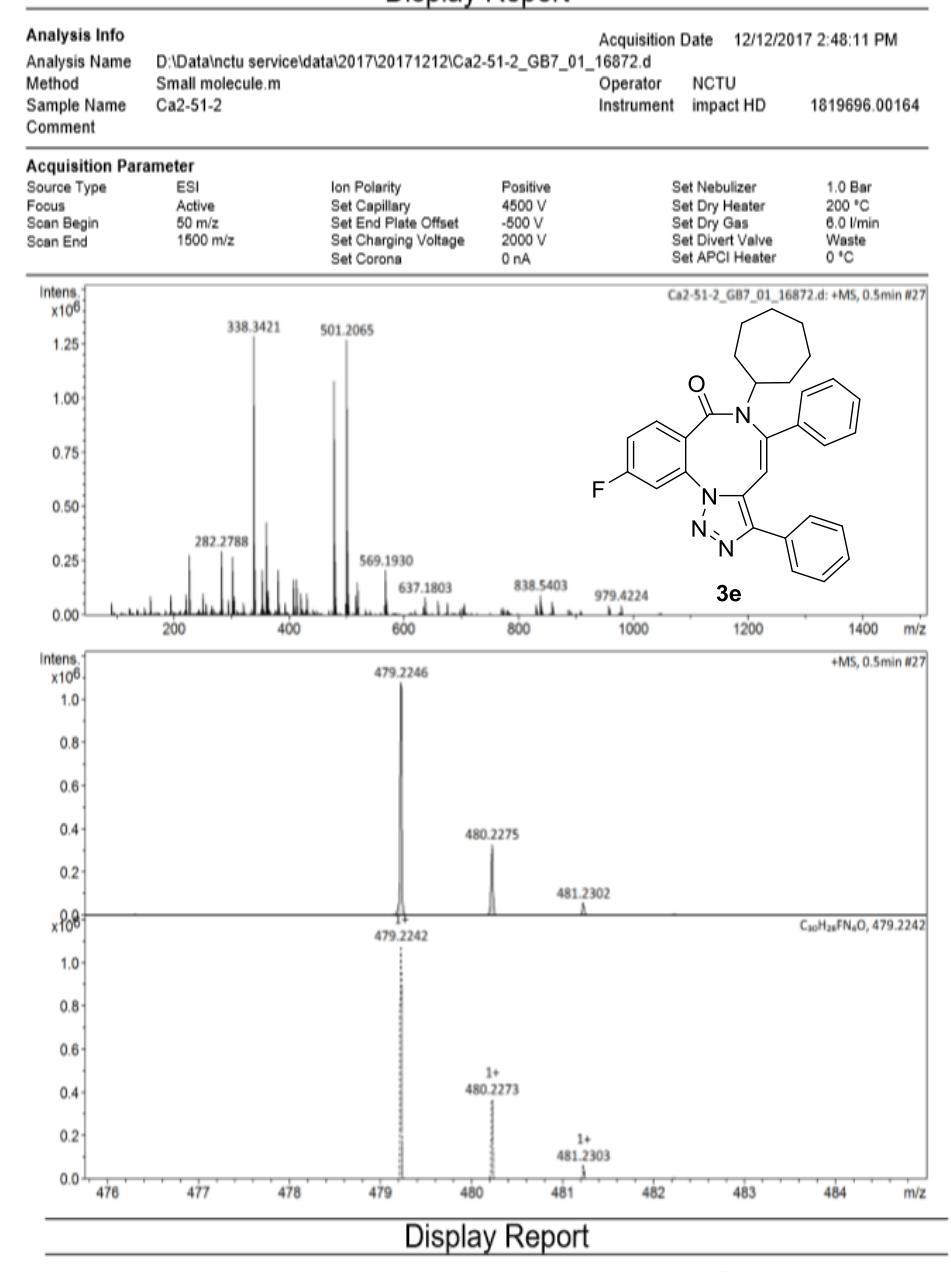

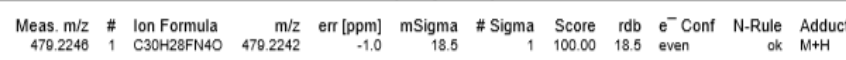

HRMS of compound $\mathbf{3 e}$ 


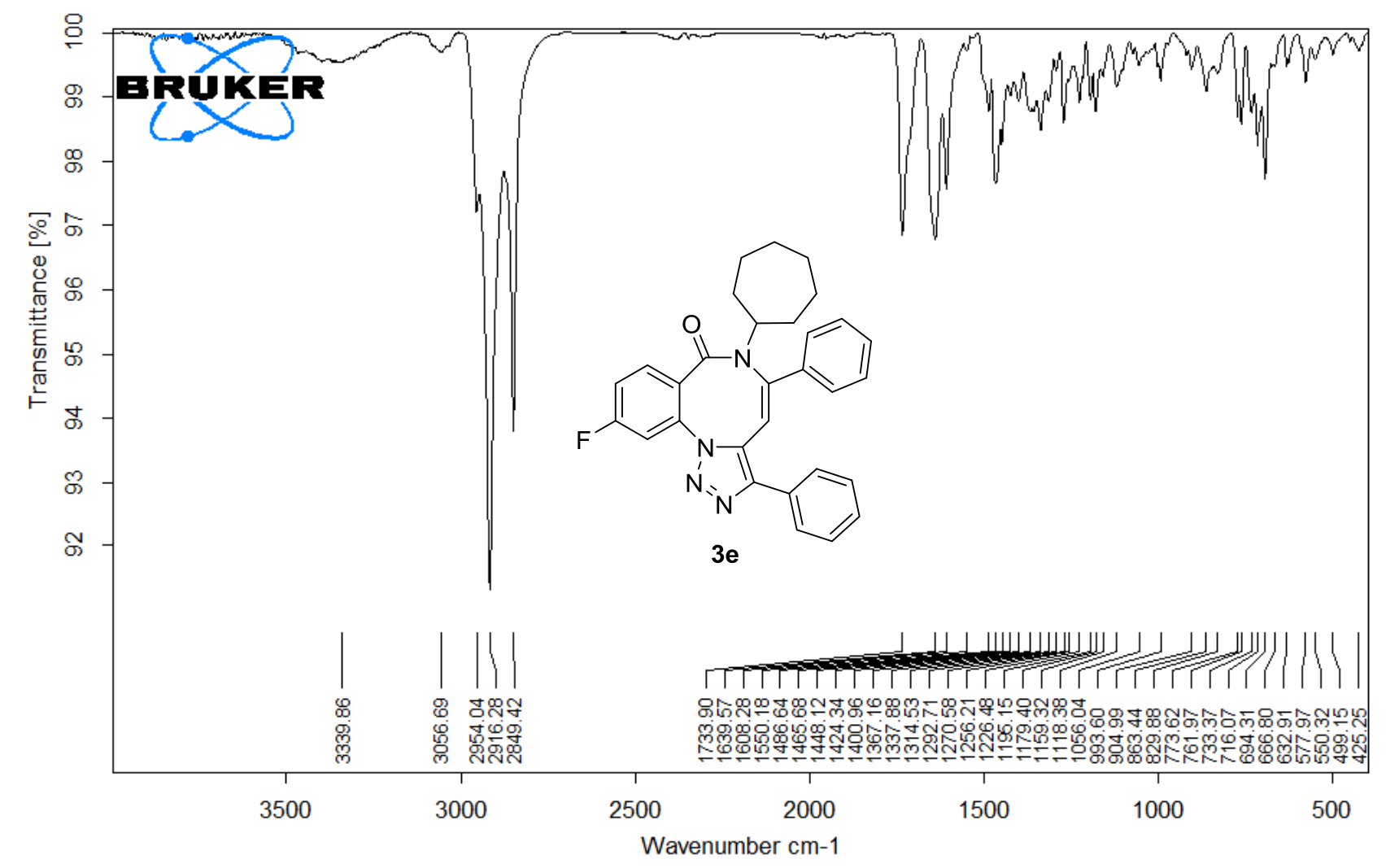

\begin{tabular}{|lcr|l|l|}
\hline D:IFTIR FILESI201808120180821IMIR_TR_DTGS_26132.0 $\quad$ MIR_TR_DTGS_26132 $\quad$ Instrument type and / or accessory & $8 / 21 / 2018$ \\
\hline
\end{tabular}

Page 1/1

IR of compound $\mathbf{3 e}$ 


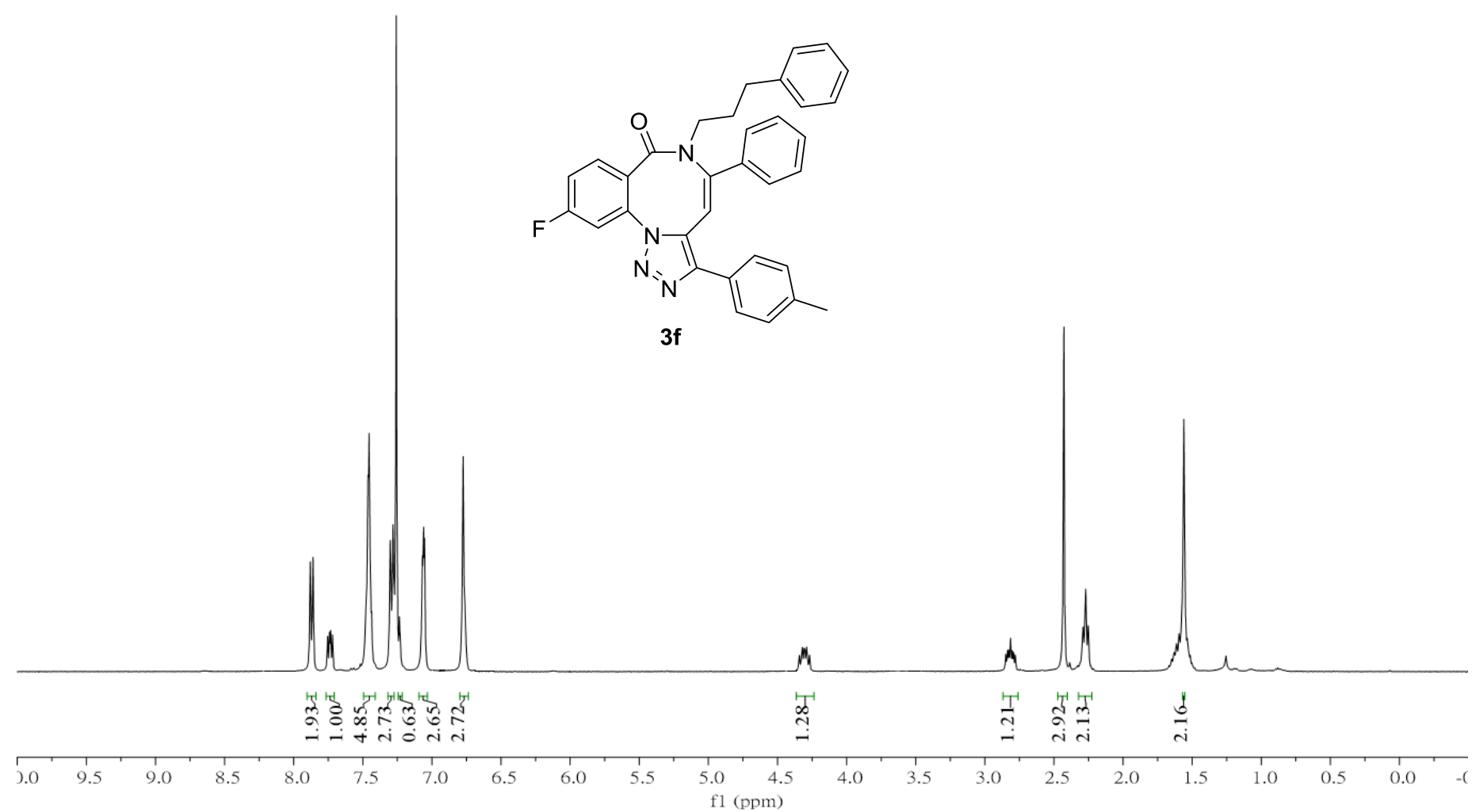

${ }^{1} \mathrm{H}$ NMR Spectrum (400 MHz) of compound $\mathbf{3 f}$ in $\mathrm{CDCl}_{3}$ 


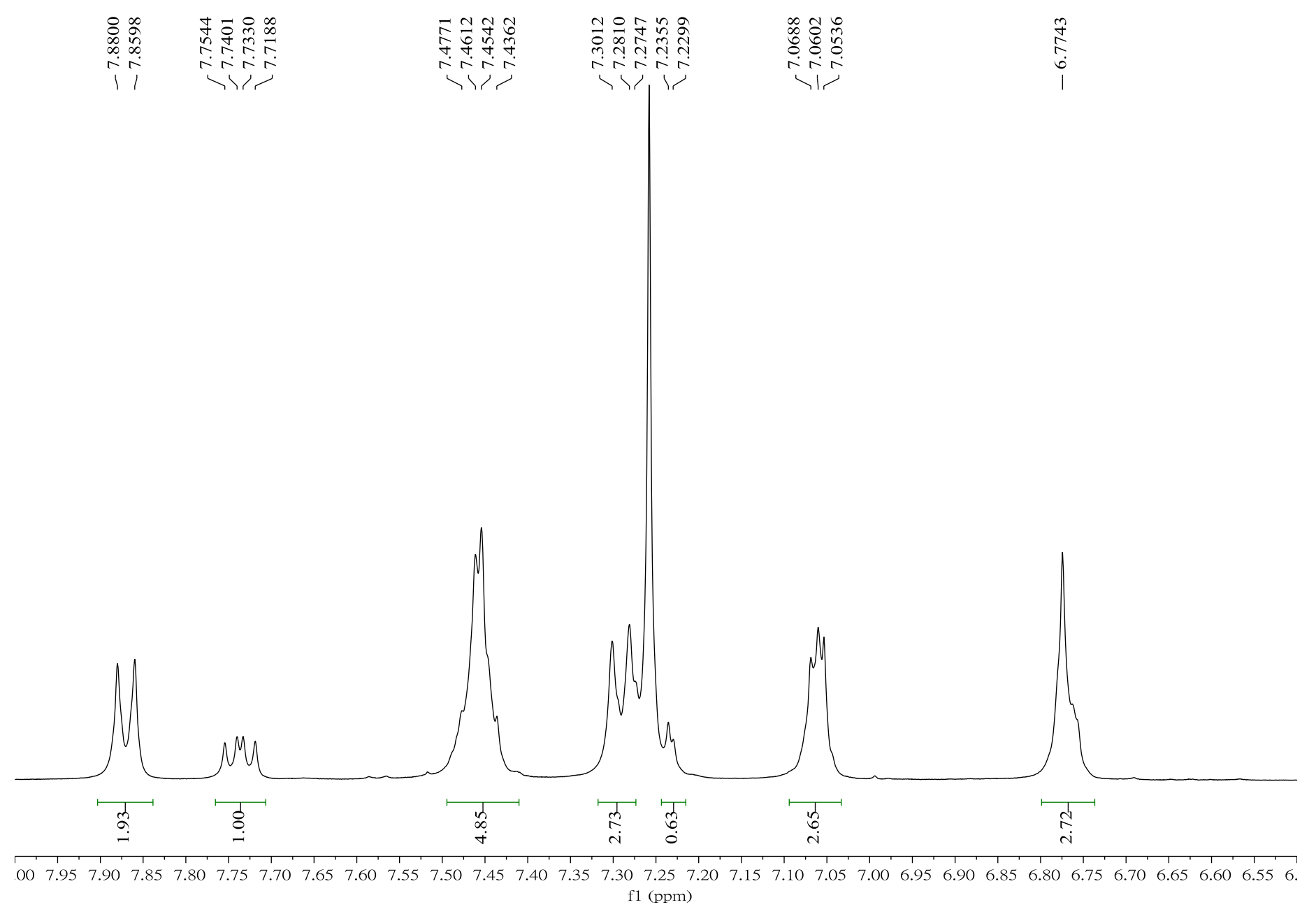

Expansion of ${ }^{1} \mathrm{H}$ NMR Spectrum $(400 \mathrm{MHz})$ of compound $3 \mathbf{f}$ in $\mathrm{CDCl}_{3}$ 

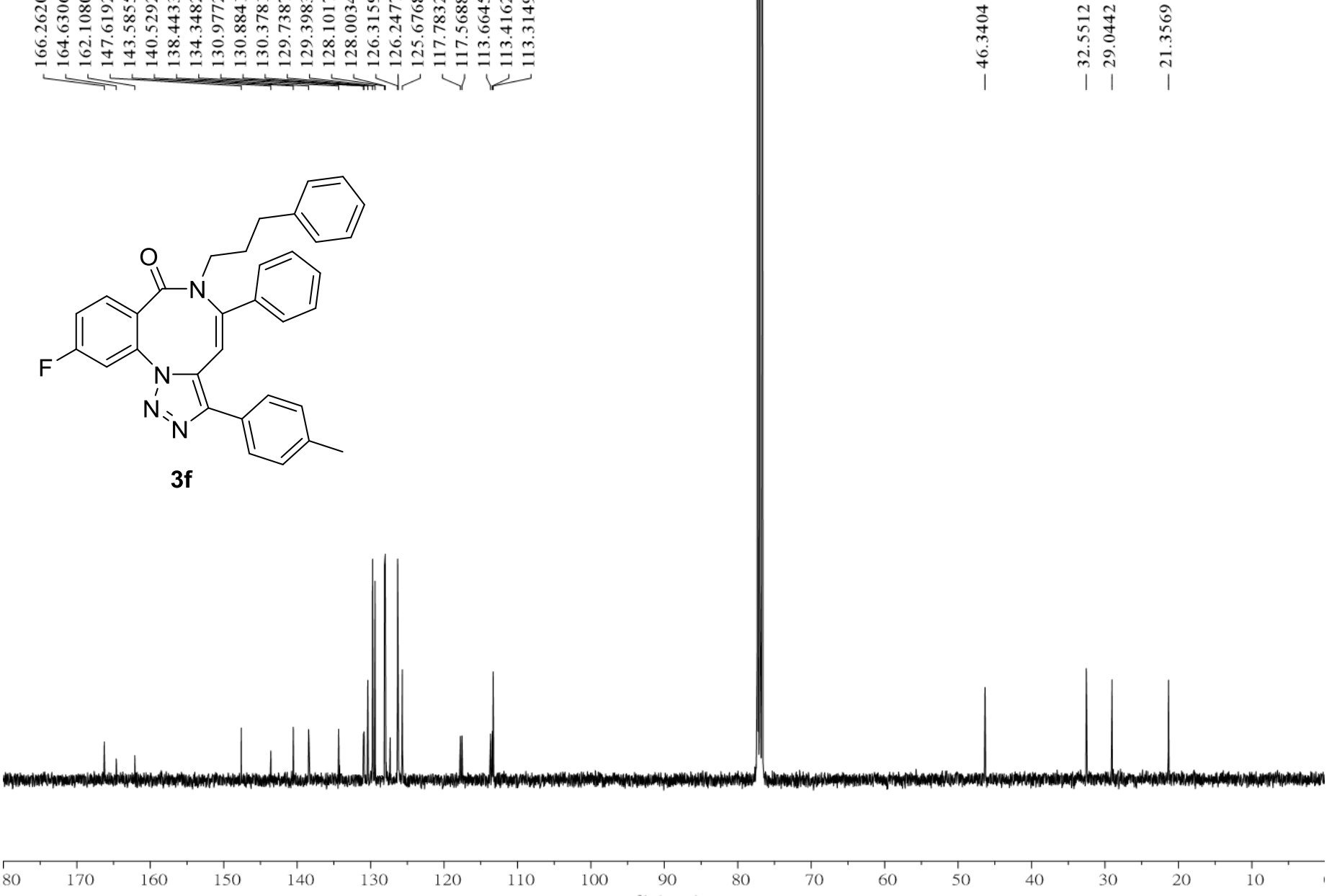

${ }^{13} \mathrm{C}$ NMR Spectrum (101 MHz) of compound $\mathbf{3 f}$ in $\mathrm{CDCl}_{3}$ 


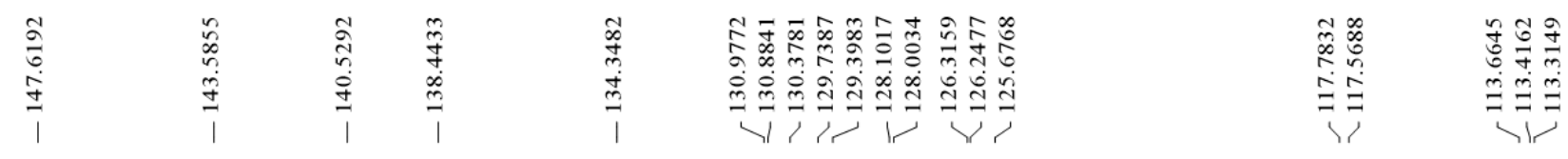

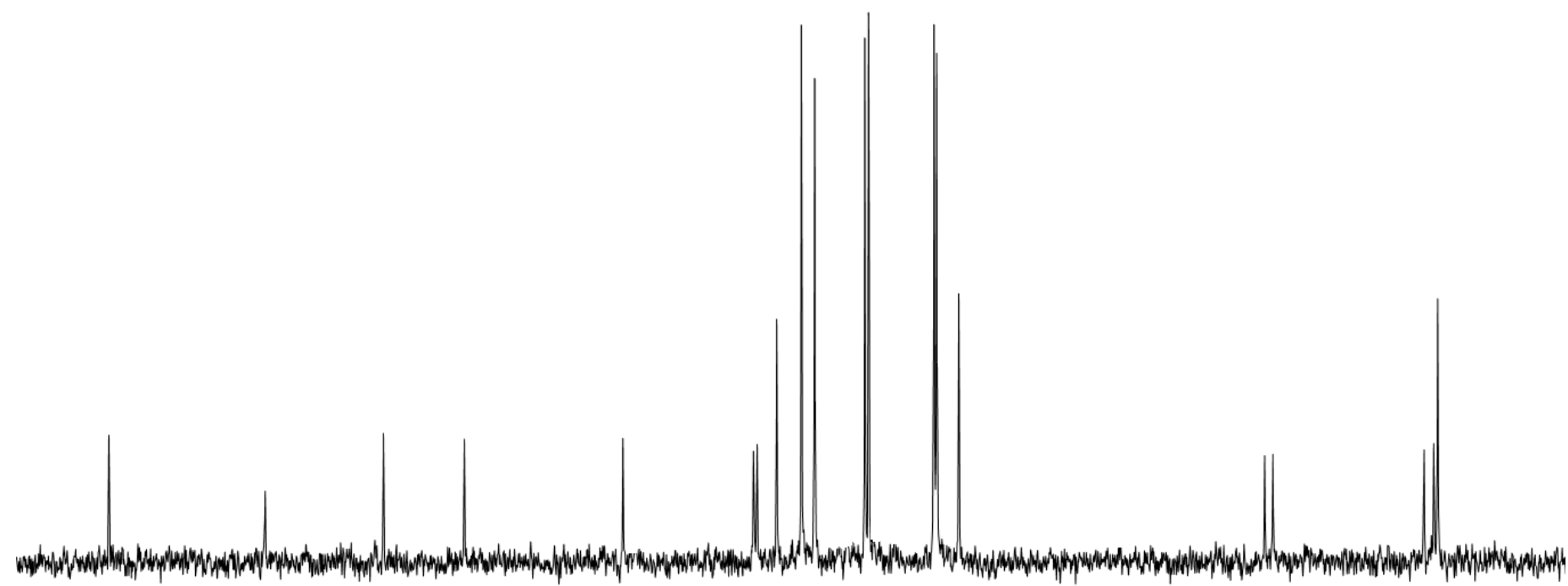

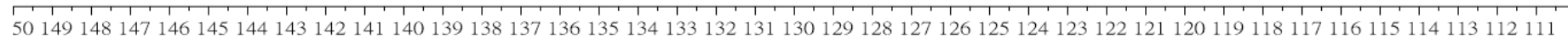
$\mathrm{fl}(\mathrm{ppm})$

Expansion of ${ }^{13} \mathrm{C}$ NMR Spectrum (101 MHz) of compound $3 \mathbf{f}$ in $\mathrm{CDCl}_{3}$ 


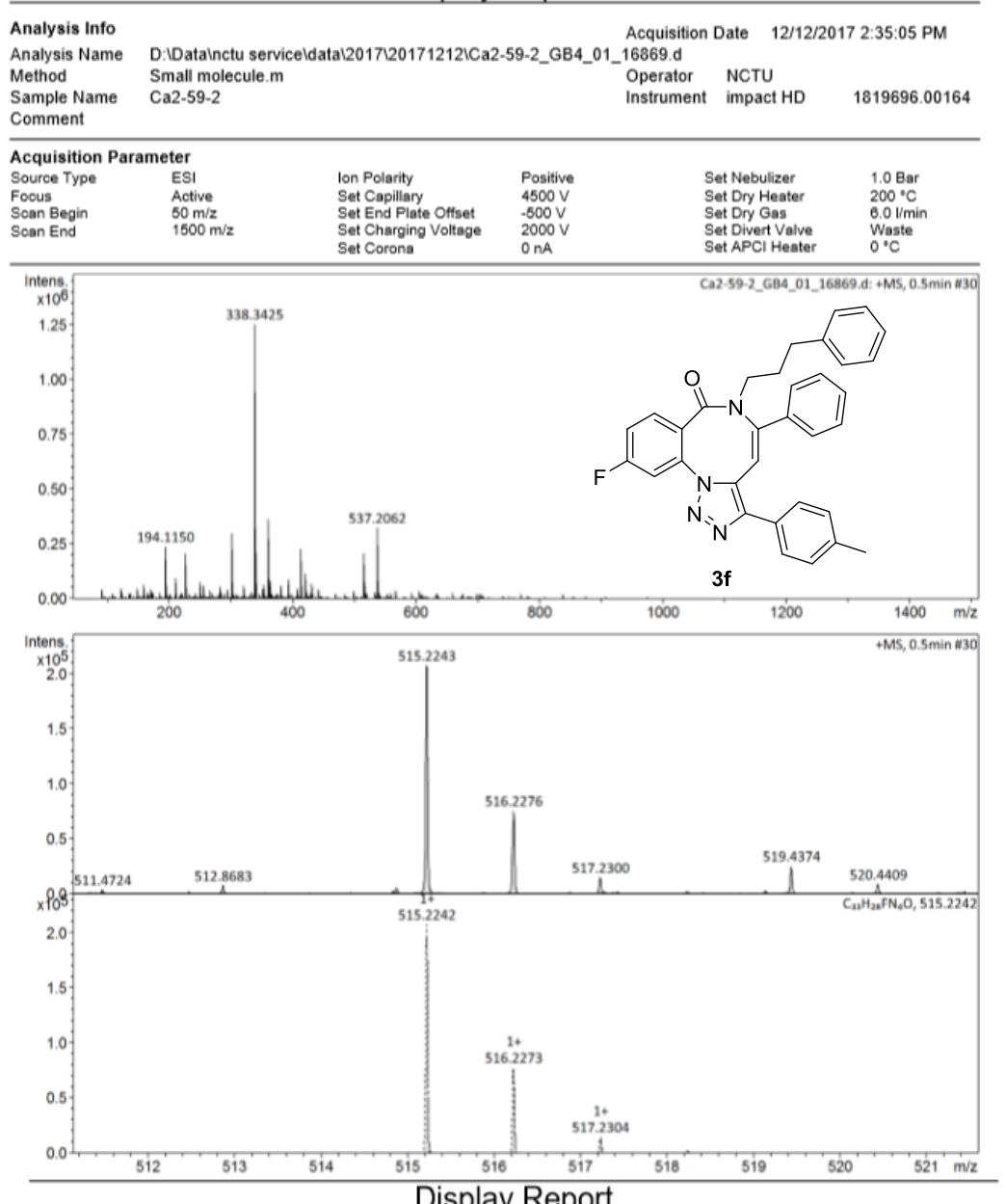

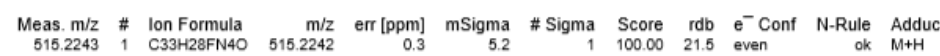

HRMS of compound $\mathbf{3 f}$ 


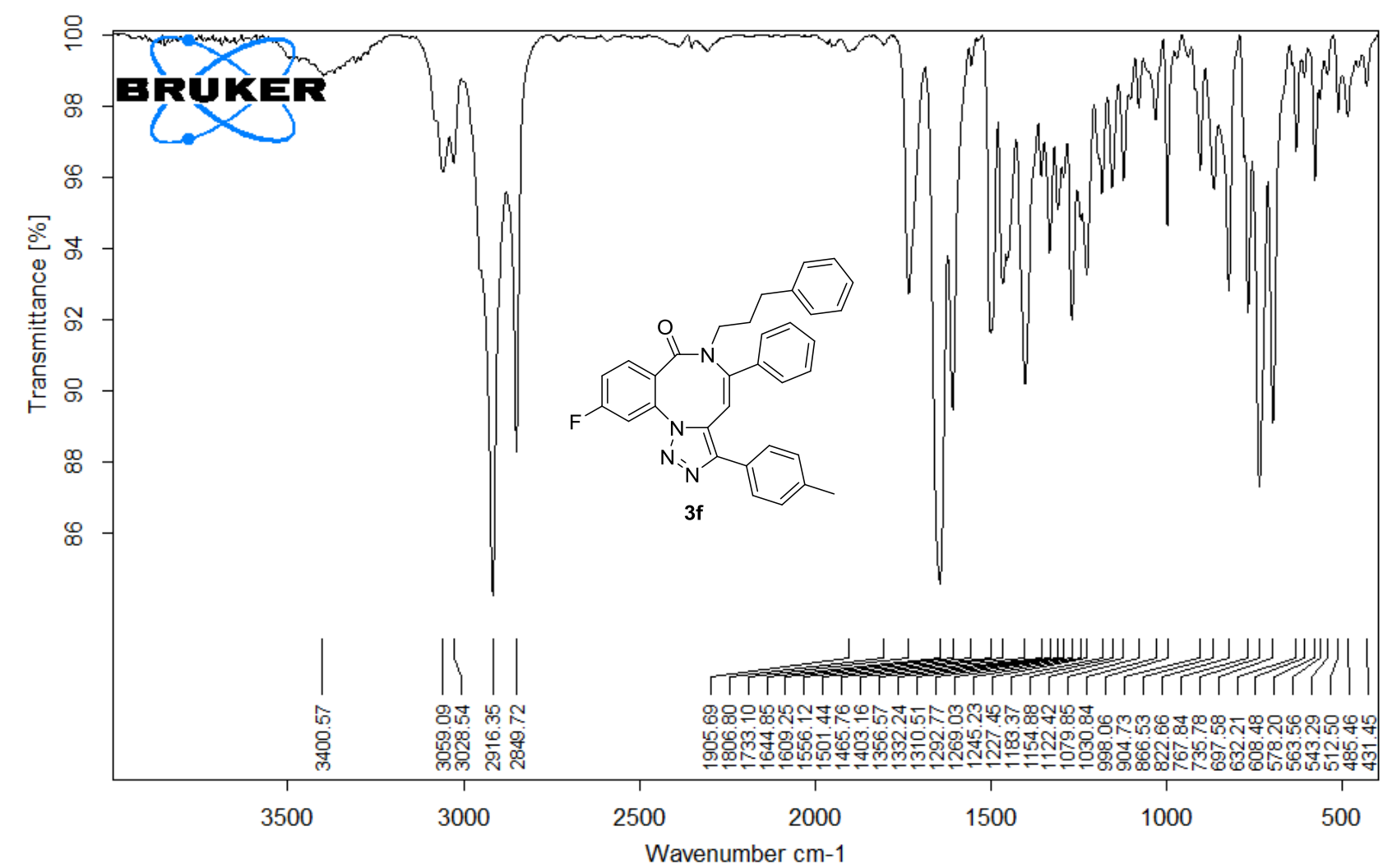

D:IFTIR FILESL201808120180821MIR_TR_DTGS_26133.0

MIR_TR_DTGS_26133

Instrument type and / or accessory

$8 / 21 / 2018$

Page 1/1

IR of compound $\mathbf{3 f}$ 


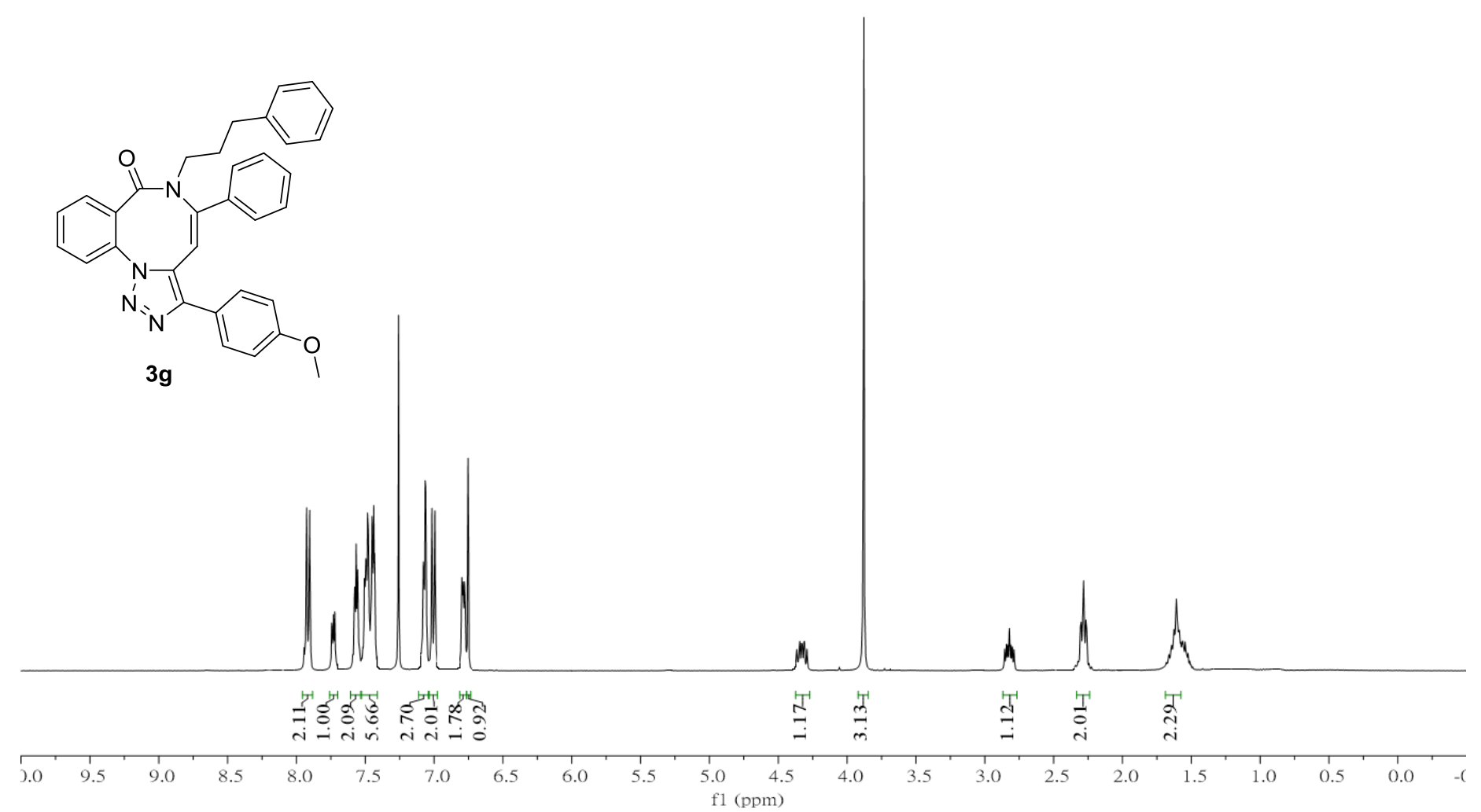

${ }^{1} \mathrm{H}$ NMR Spectrum (400 MHz) of compound $\mathbf{3 g}$ in $\mathrm{CDCl}_{3}$ 


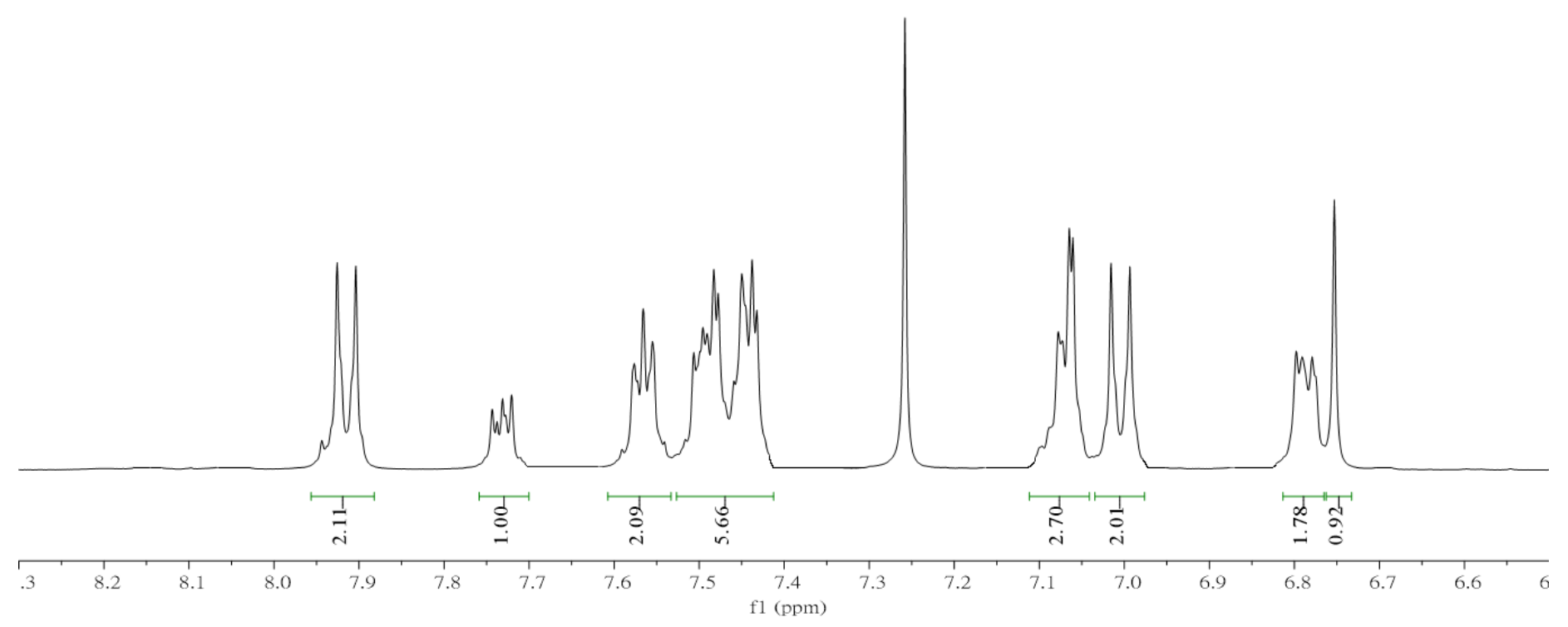

Expansion of ${ }^{1} \mathrm{H}$ NMR Spectrum (400 MHz) of compound $3 g$ in $\mathrm{CDCl}_{3}$ 


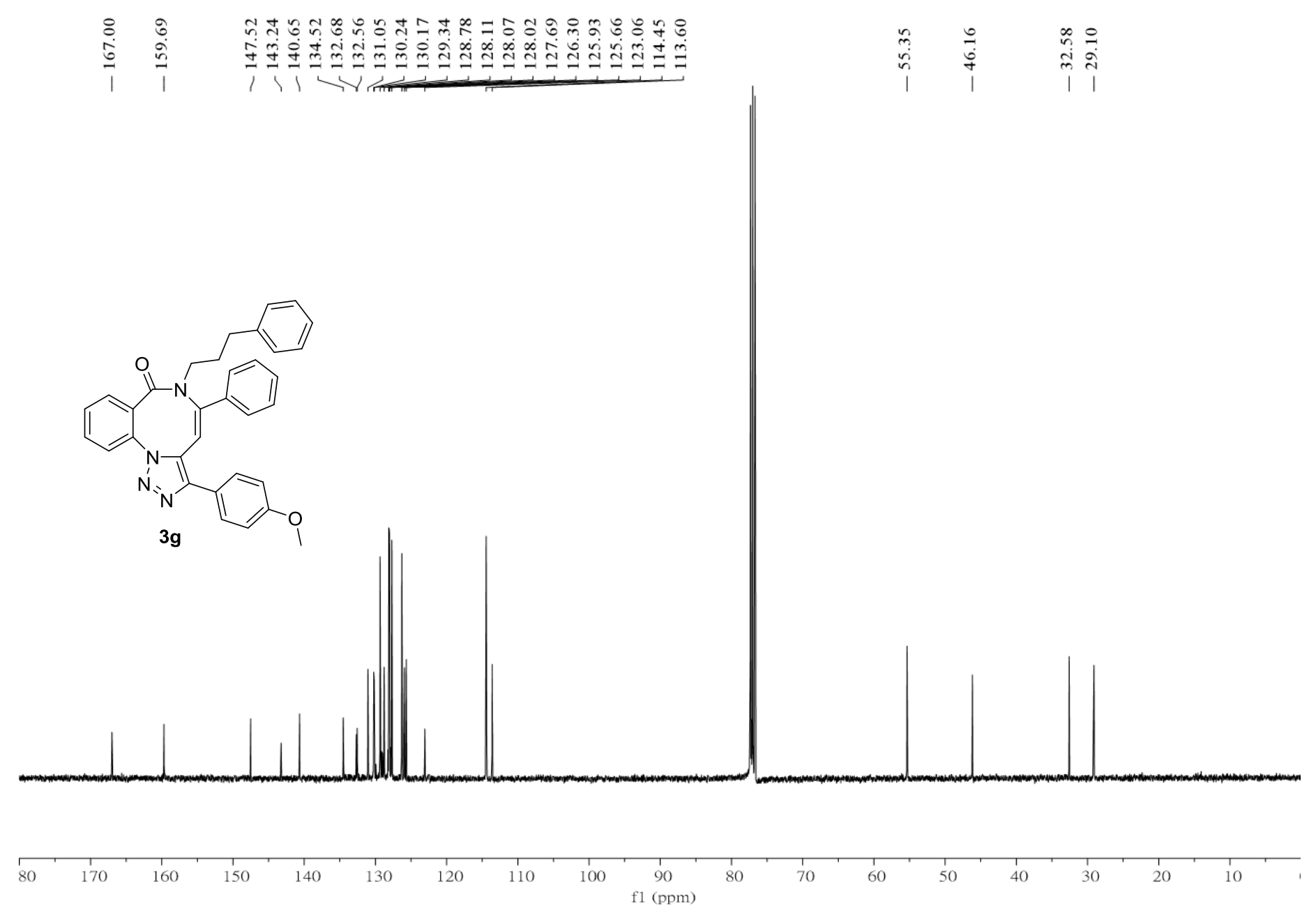

${ }^{13} \mathrm{C}$ NMR Spectrum (101 MHz) of compound $\mathbf{3 g}$ in $\mathrm{CDCl}_{3}$ 


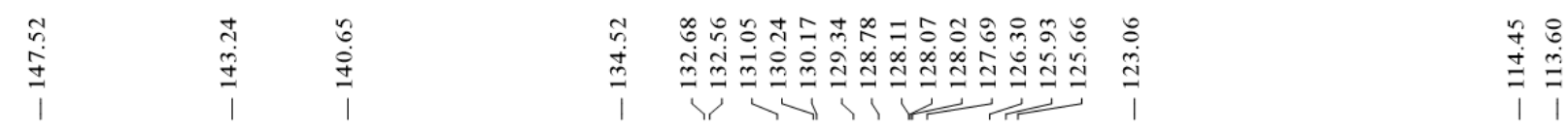

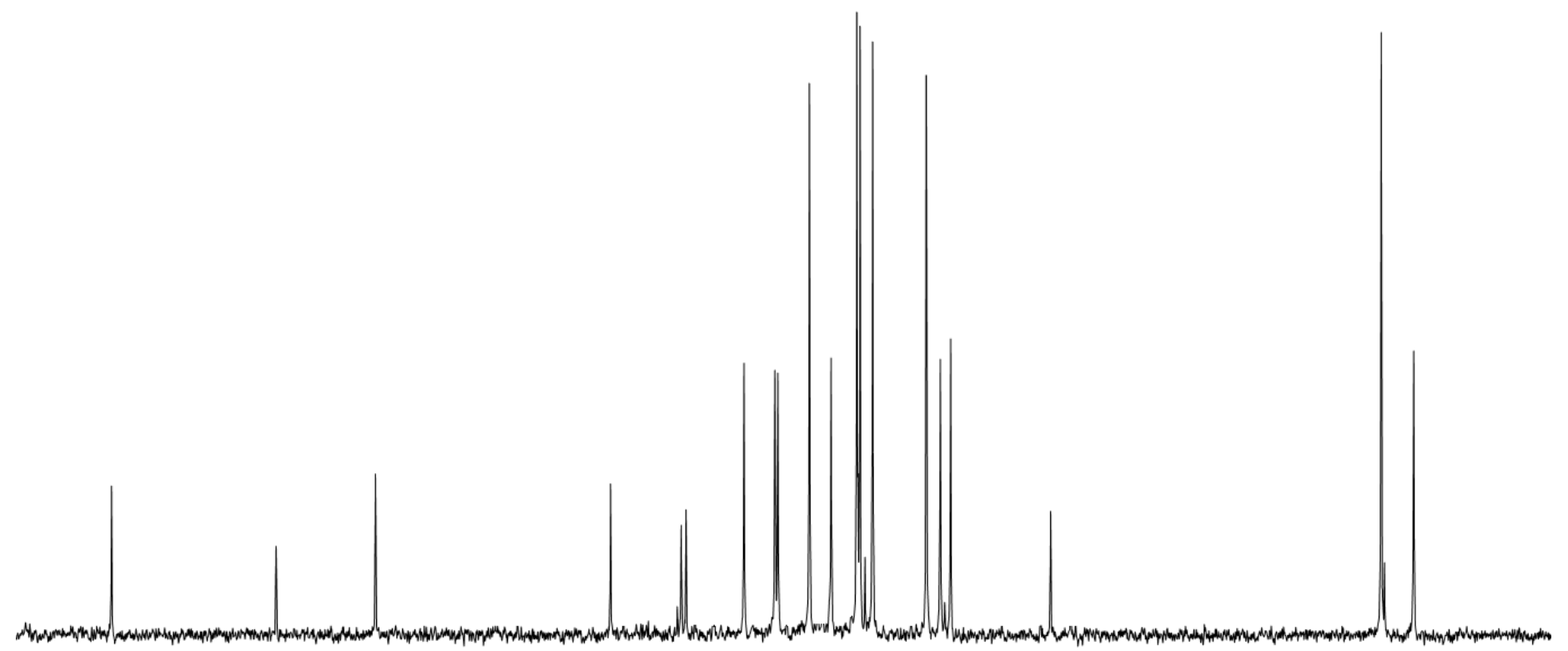

5014914814714614514414314214114013913813713613513413313213113012912812712612512412312212112011911811716115114113112111 $\mathrm{fl}(\mathrm{ppm})$

Expansion of ${ }^{13} \mathrm{C}$ NMR Spectrum (101 MHz) of compound $\mathbf{3 g}$ in $\mathrm{CDCl}_{3}$ 


\section{Display Report}

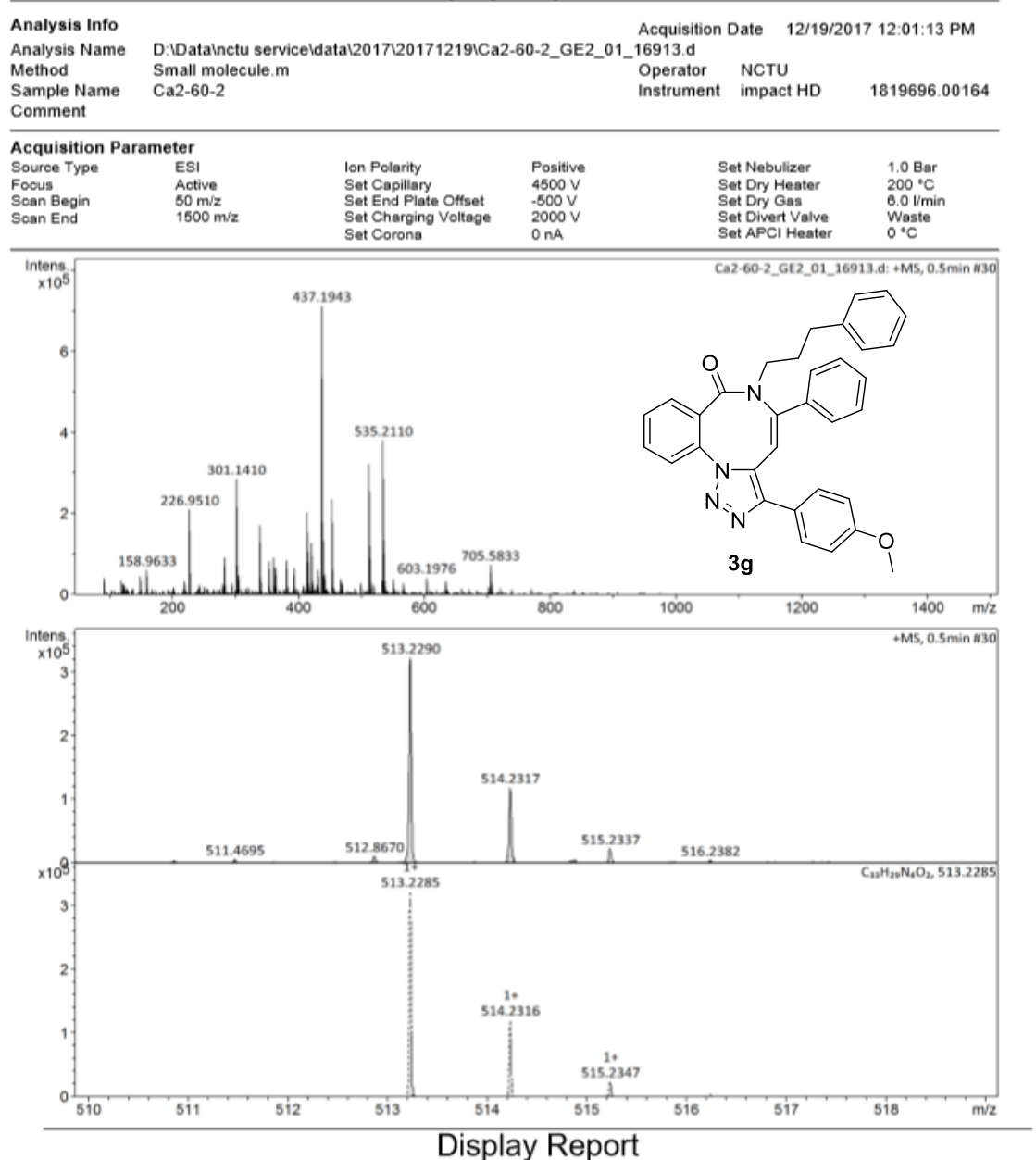

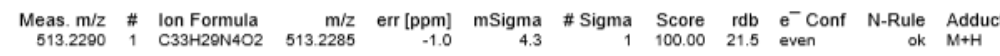

HRMS of compound $\mathbf{3 g}$ 


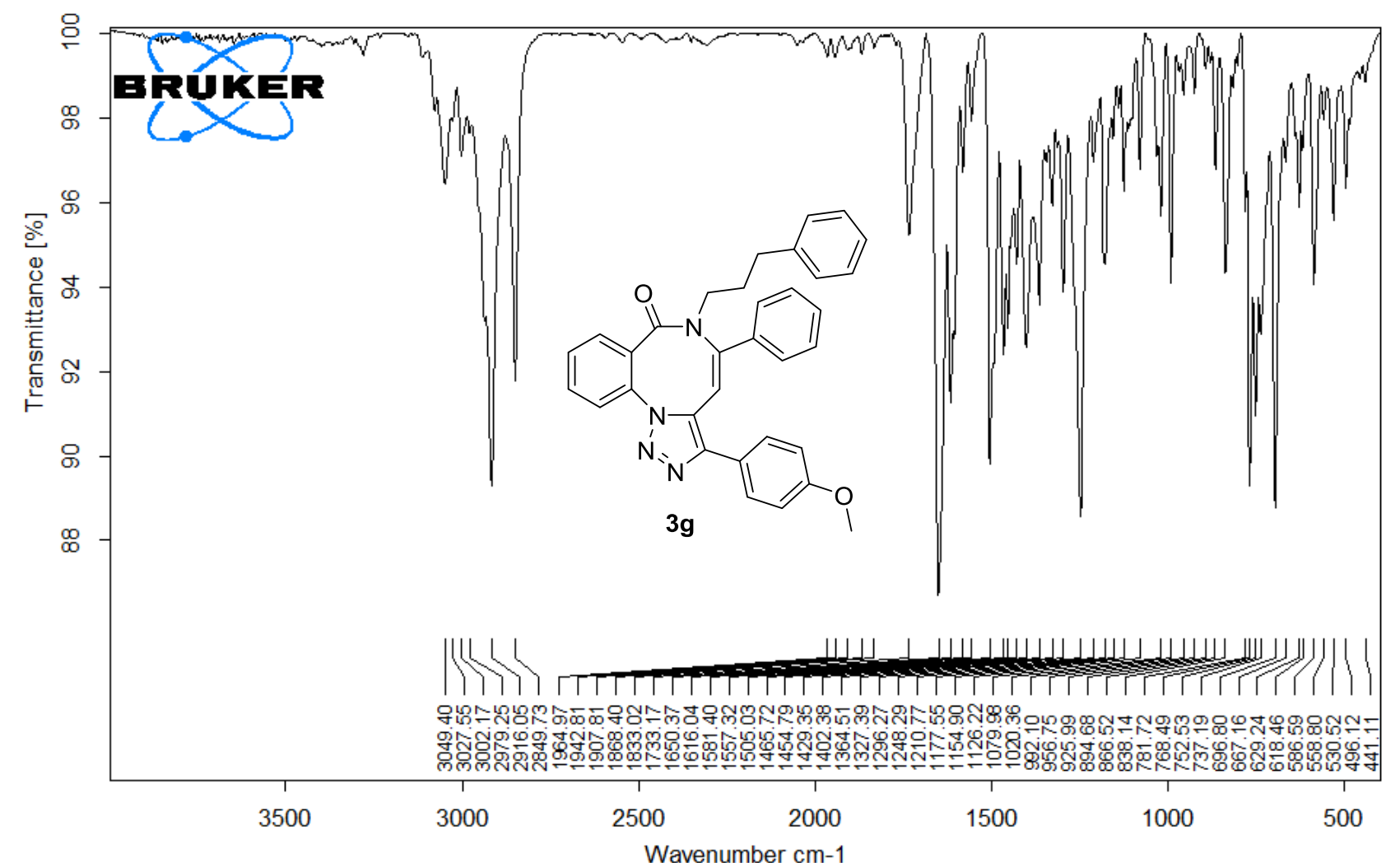

\begin{tabular}{|lcc|c|}
\hline D:IFTIR FILESI201808120180821MIR_TR_DTGS_26134.0 $\quad$ MIR_TR_DTGS_26134 $\quad$ Instrument type and / or accessory & $8 / 21 / 2018$ \\
\hline
\end{tabular}

Page 1/1

IR of compound $\mathbf{3 g}$ 


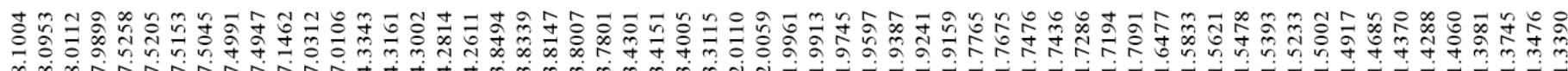

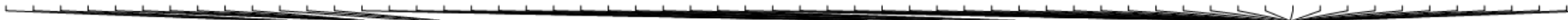

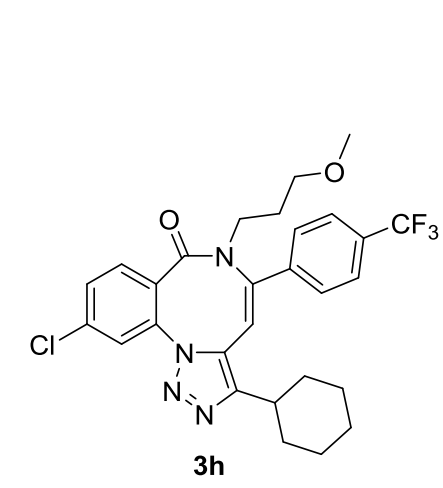

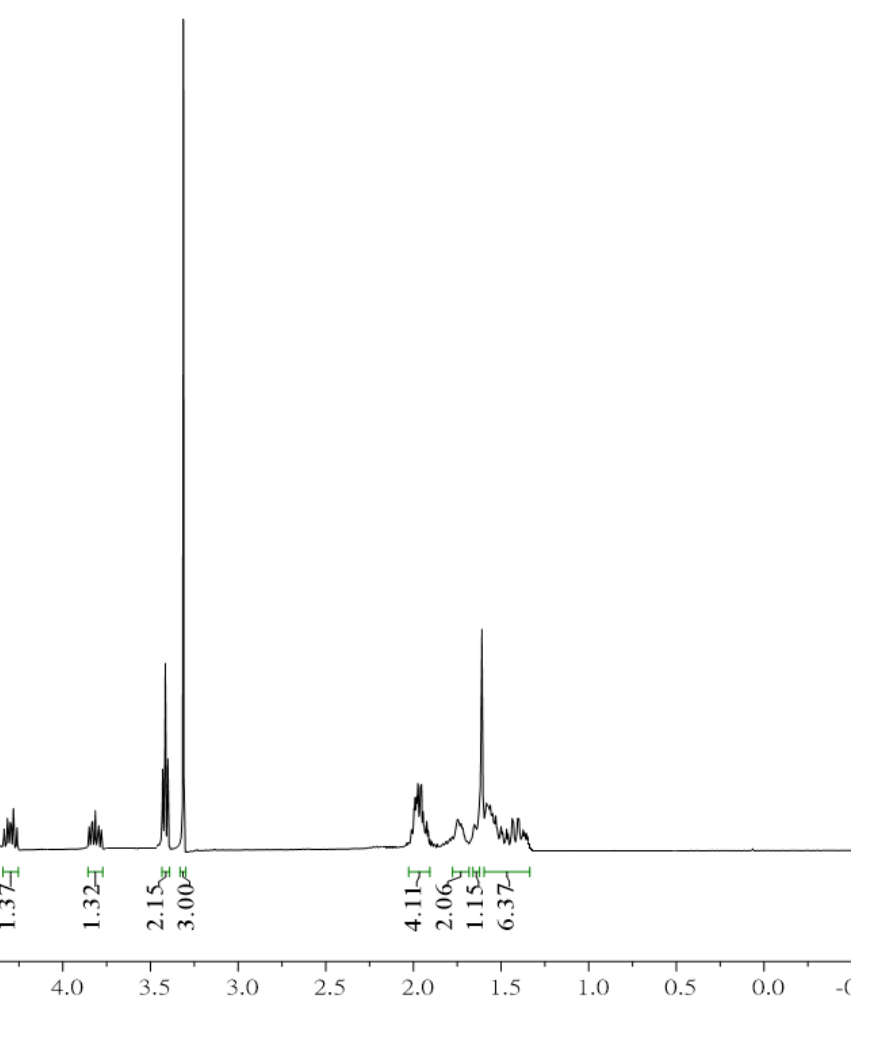

${ }^{1} \mathrm{H}$ NMR Spectrum (400 MHz) of compound $\mathbf{3 h}$ in $\mathrm{CDCl}_{3}$ 

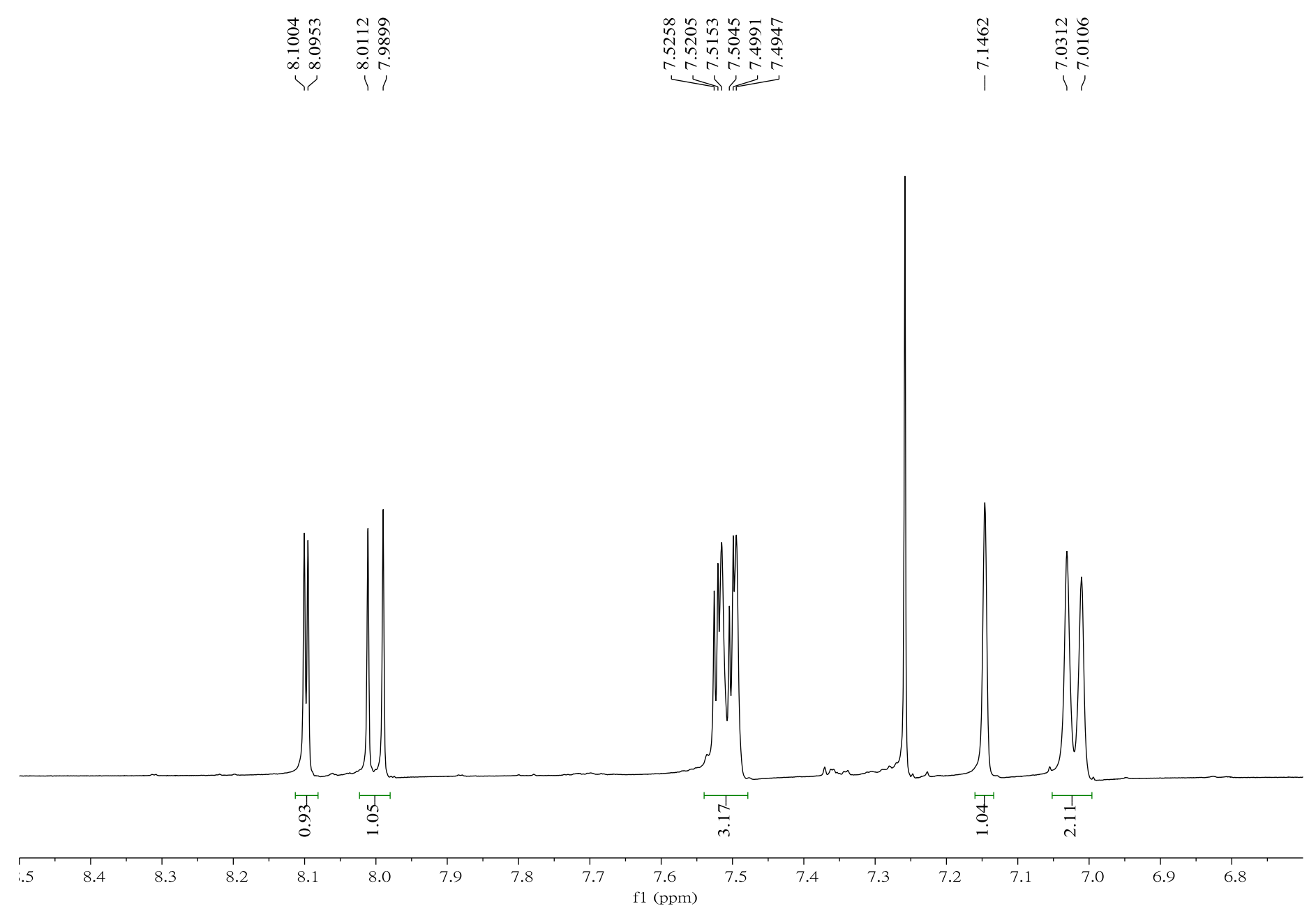

Expansion of ${ }^{1} \mathrm{H}$ NMR Spectrum (400 MHz) of compound $\mathbf{3 h}$ in $\mathrm{CDCl}_{3}$ 


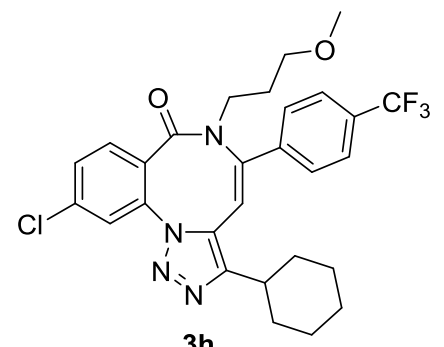

3h
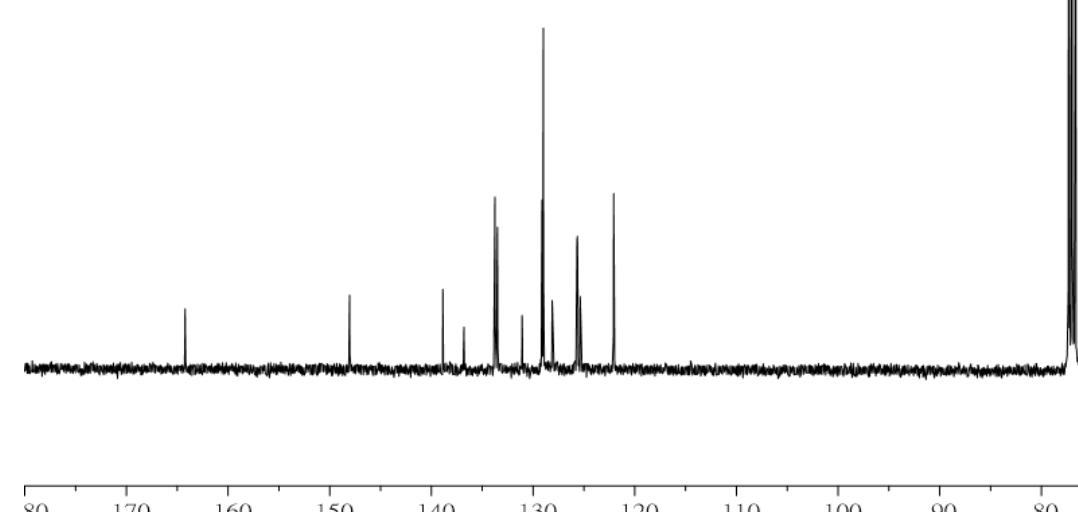

130

20

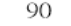

$80 \quad 70$

60

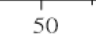

40

20

${ }^{13} \mathrm{C}$ NMR Spectrum (101 MHz) of compound $\mathbf{3 h}$ in $\mathrm{CDCl}_{3}$ 


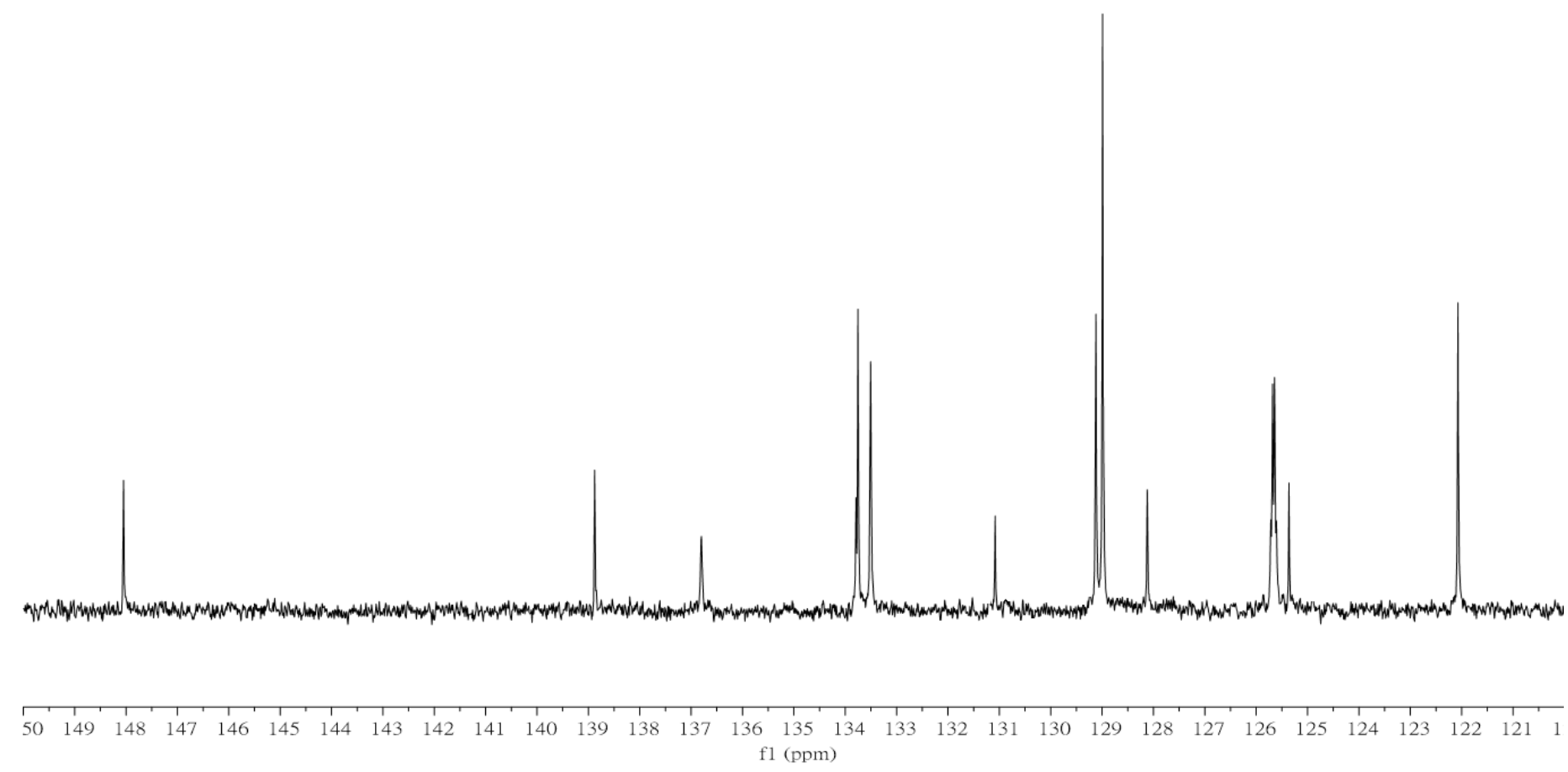

Expansion of ${ }^{13} \mathrm{C}$ NMR Spectrum $(101 \mathrm{MHz})$ of compound $\mathbf{3 h}$ in $\mathrm{CDCl}_{3}$ 
Display Report

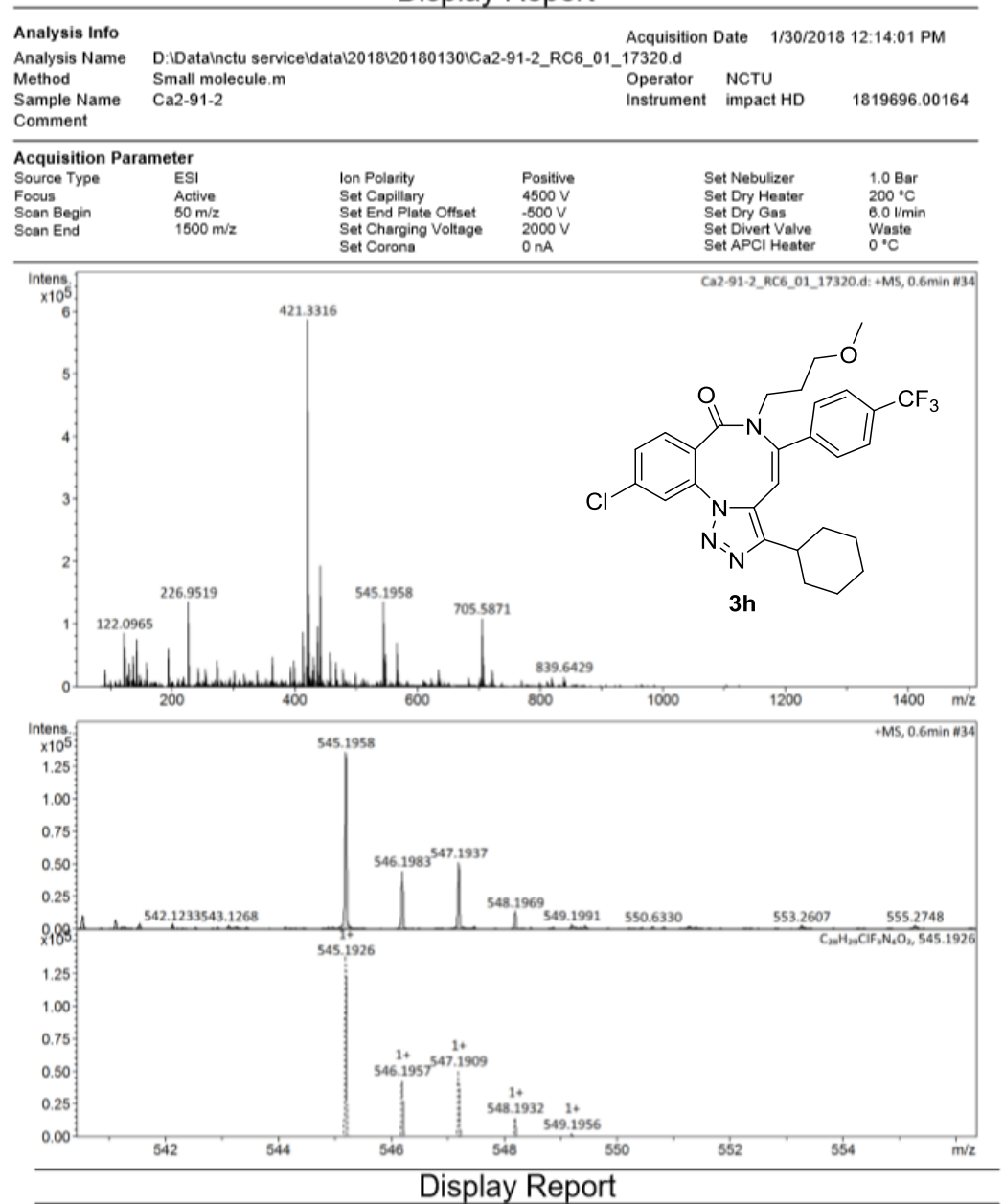

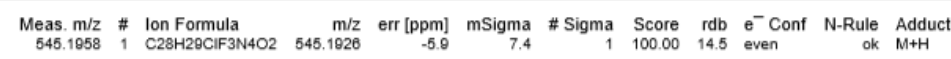

HRMS of compound $\mathbf{3 h}$ 


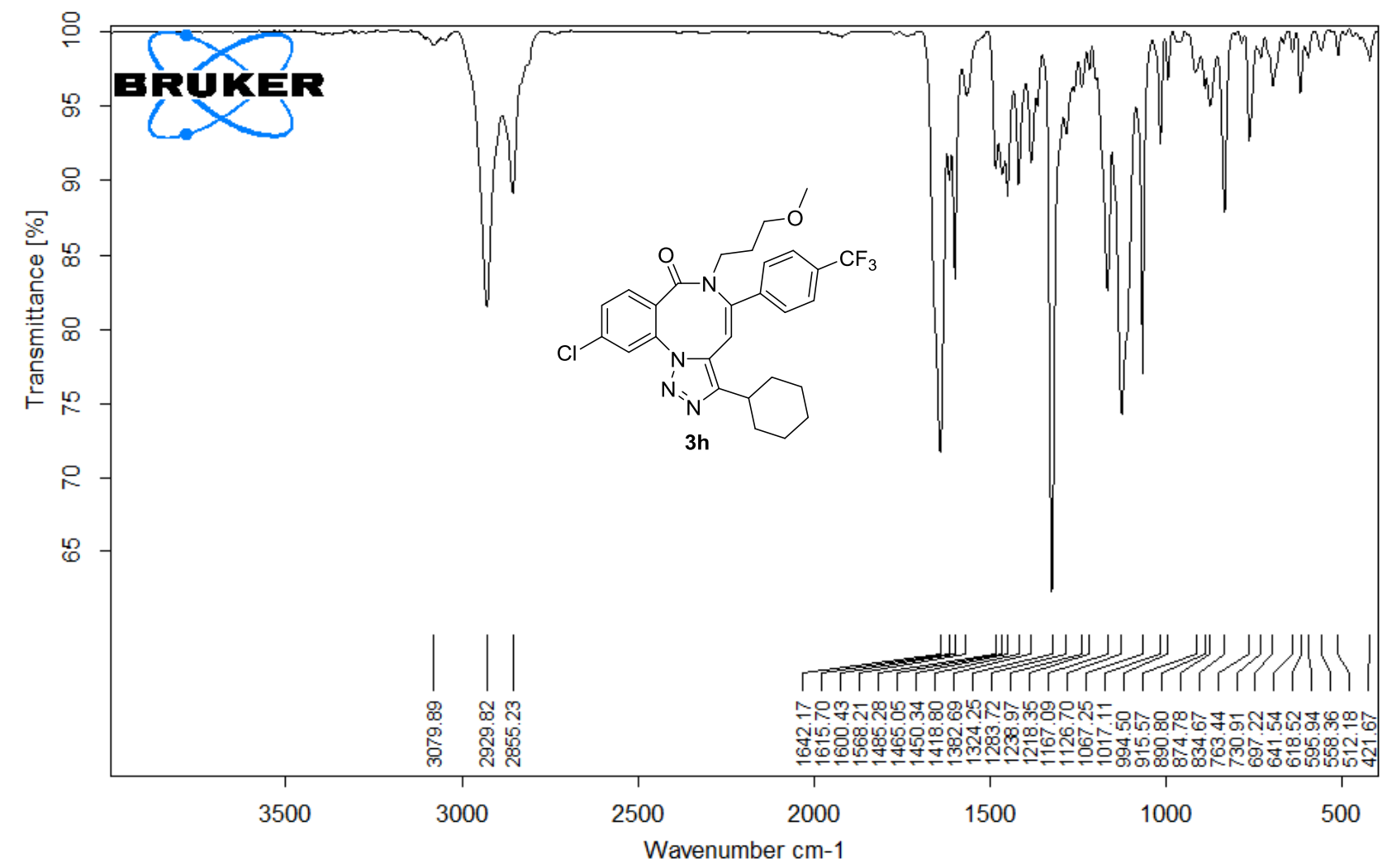

\begin{tabular}{|llc|l|}
\hline D:IFTIR FILESL201808120180821MIR_TR_DTGS_26135.0 $\quad$ MIR_TR_DTGS_26135 $\quad$ Instrument type and / or accessory & $8 / 21 / 2018$ \\
\hline
\end{tabular}

Page 1/1

IR of compound $\mathbf{3 h}$ 


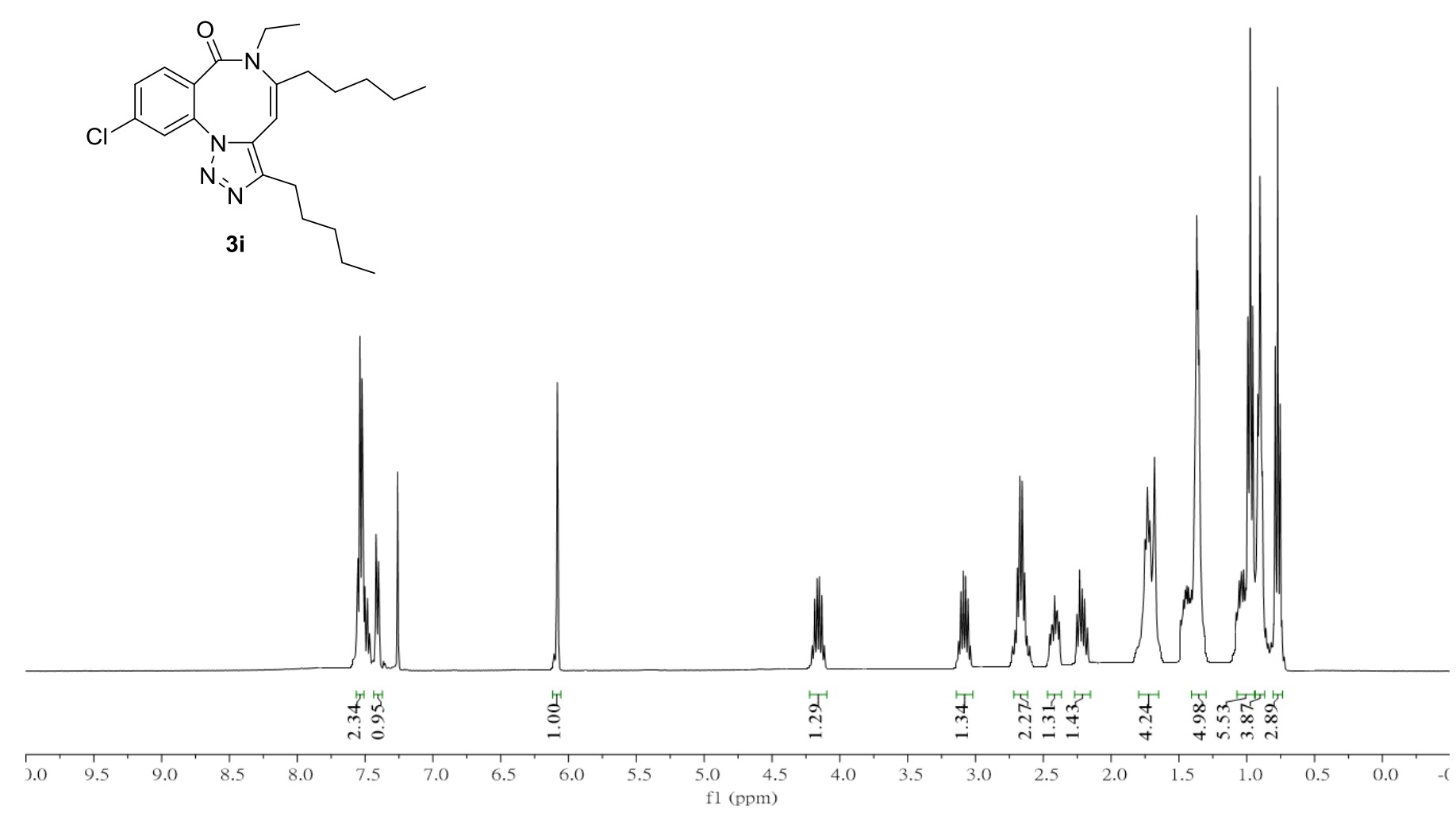

${ }^{1} \mathrm{H}$ NMR Spectrum (400 MHz) of compound $\mathbf{3 i}$ in $\mathrm{CDCl}_{3}$ 

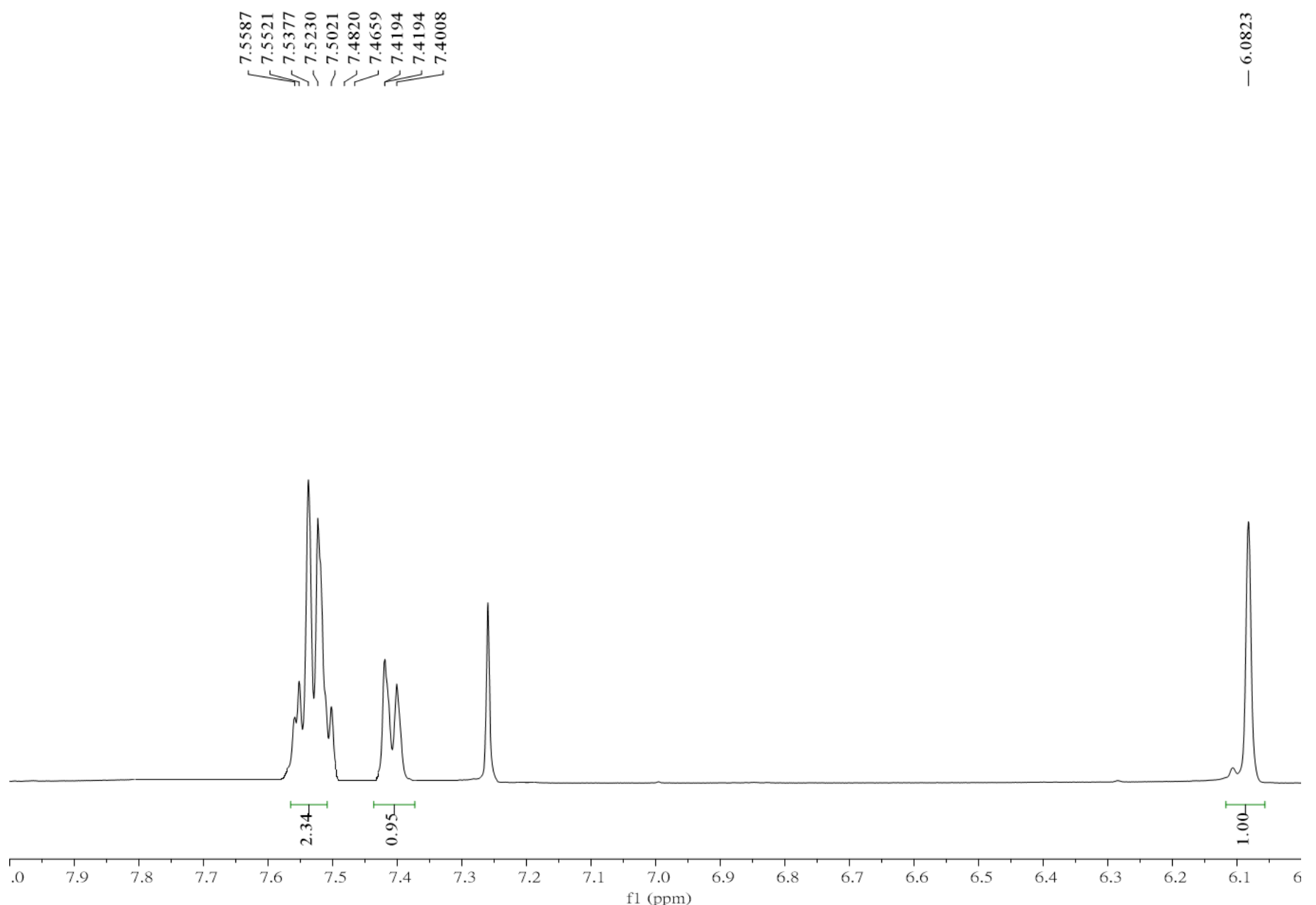

Expansion of ${ }^{1} \mathrm{H}$ NMR Spectrum (400 MHz) of compound $3 \mathbf{i}$ in $\mathrm{CDCl}_{3}$ 

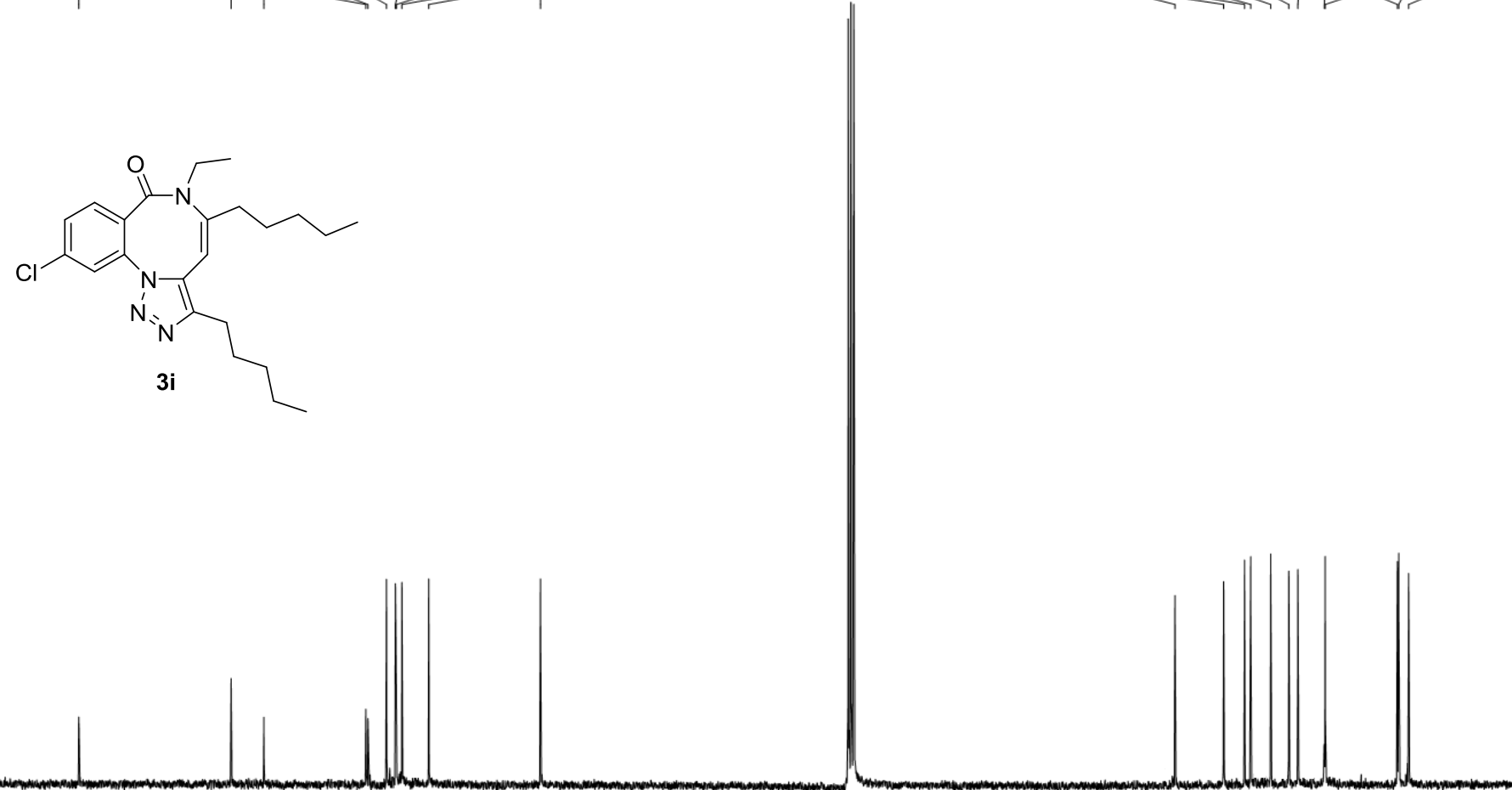

80

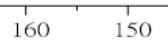

140

120

100

$80 \quad 70$

60

50

${ }^{13} \mathrm{C}$ NMR Spectrum (101 MHz) of compound $\mathbf{3 i}$ in $\mathrm{CDCl}_{3}$ 


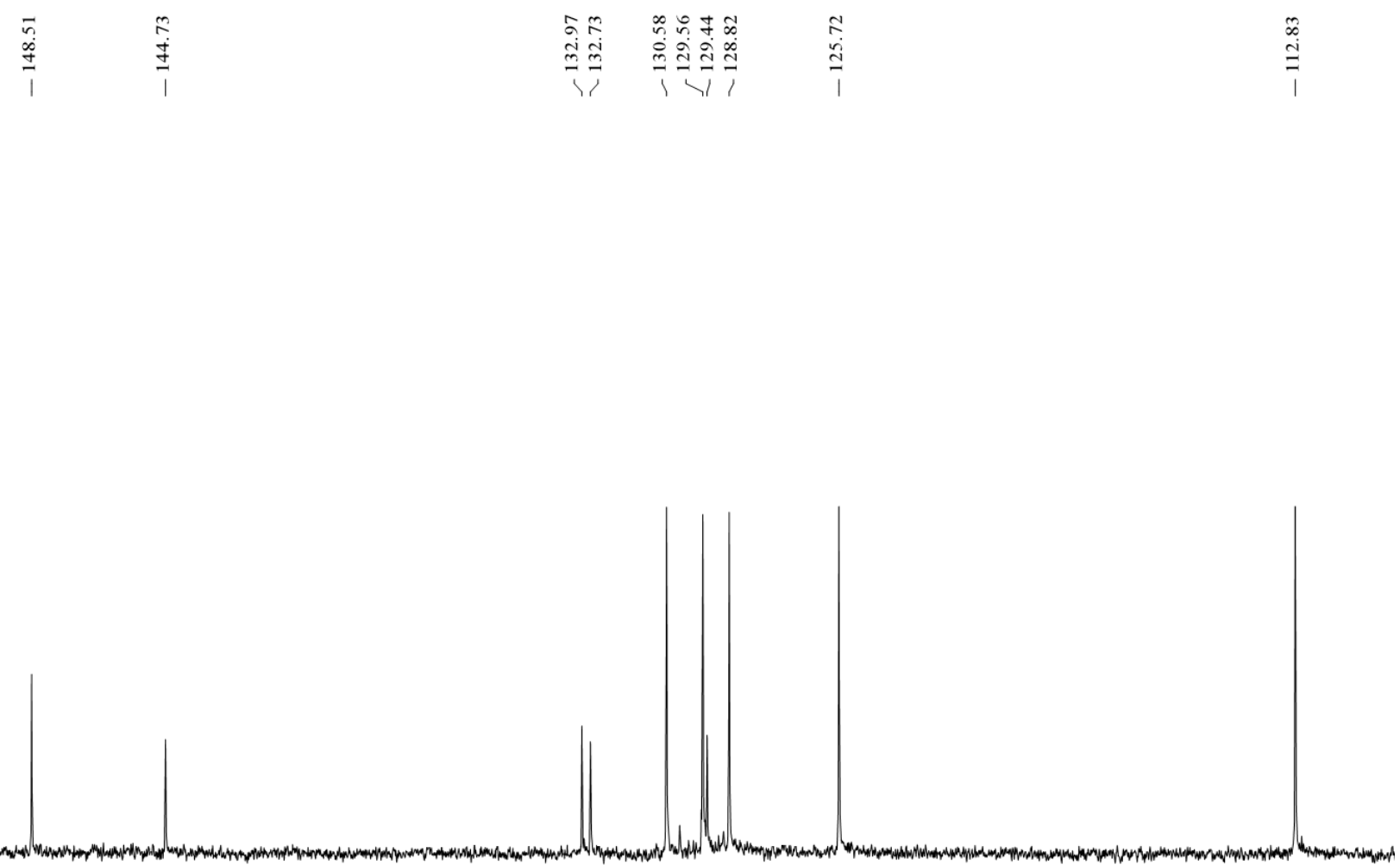

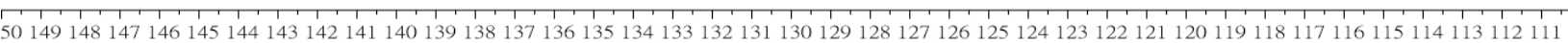

Expansion of ${ }^{13} \mathrm{C}$ NMR Spectrum (101 MHz) of compound $3 \mathbf{3 i n} \mathrm{CDCl}_{3}$ 


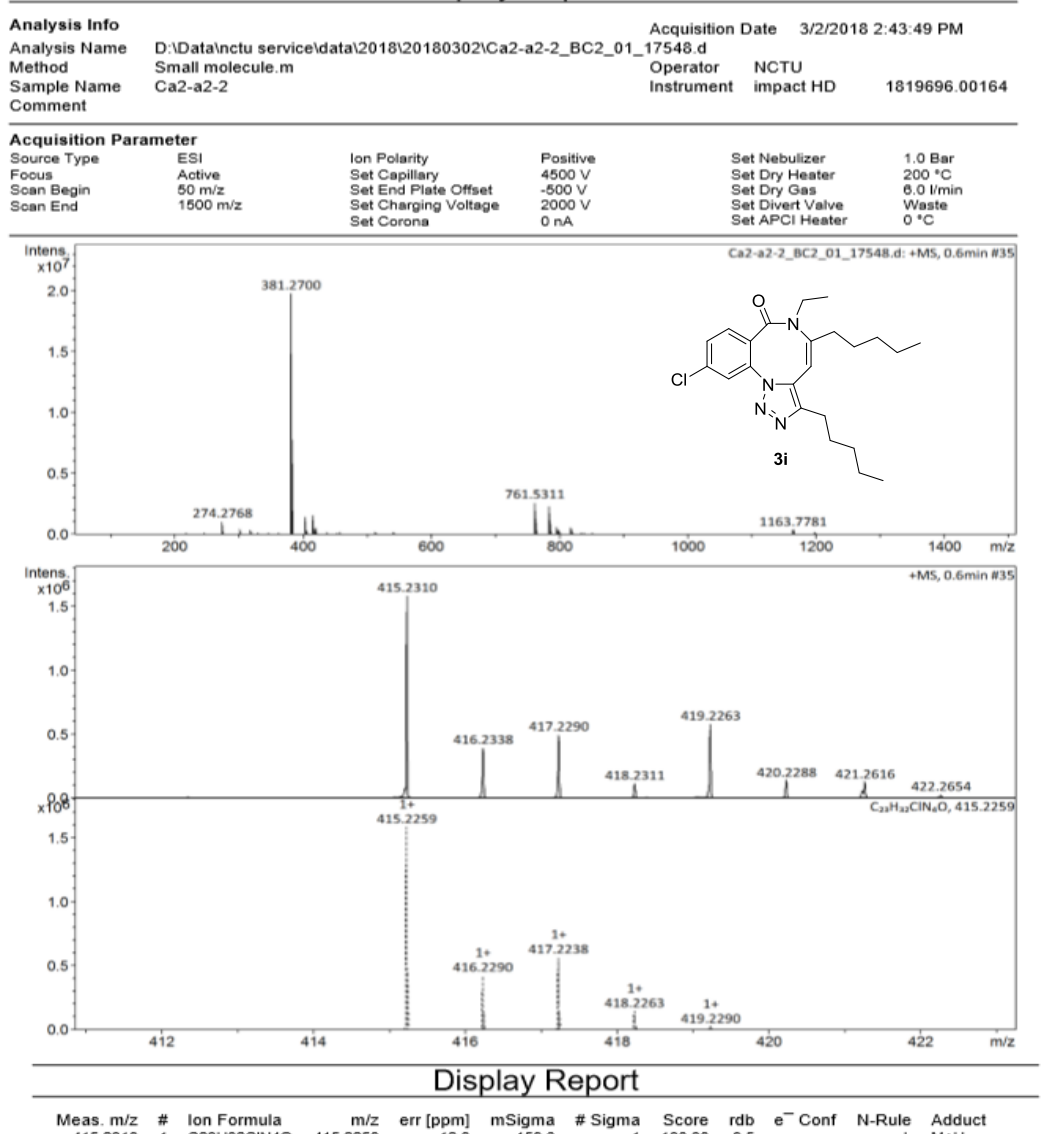

HRMS of compound $\mathbf{3 i}$ 


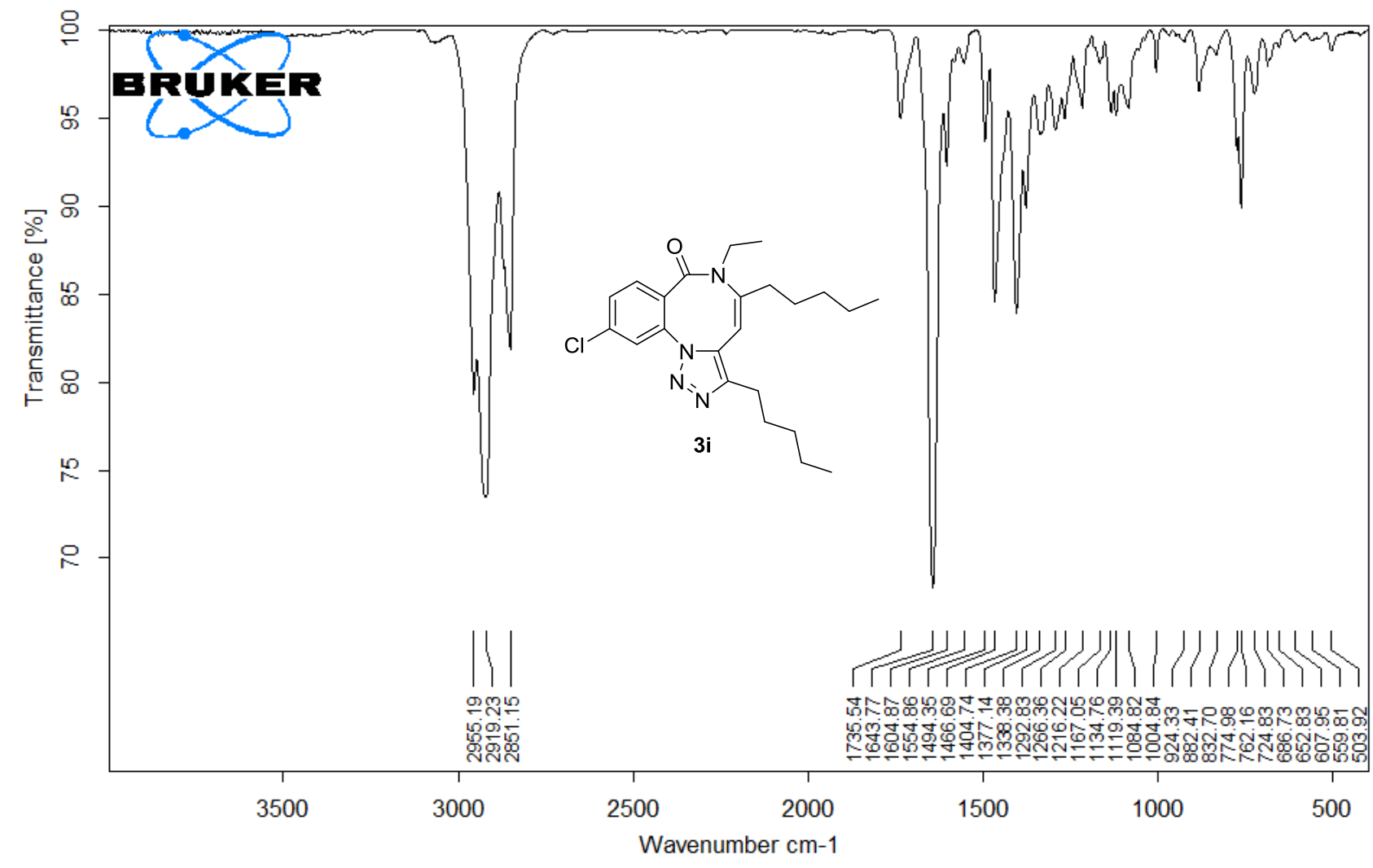

\begin{tabular}{|lcl|l|}
\hline D:IFTIR FILESI201808120180821MIR_TR_DTGS_26136.0 $\quad$ MIR_TR_DTGS_26136 $\quad$ Instrument type and / or accessory & $8 / 21 / 2018$ \\
\hline
\end{tabular}

Page 1/1

IR of compound $\mathbf{3 i}$ 


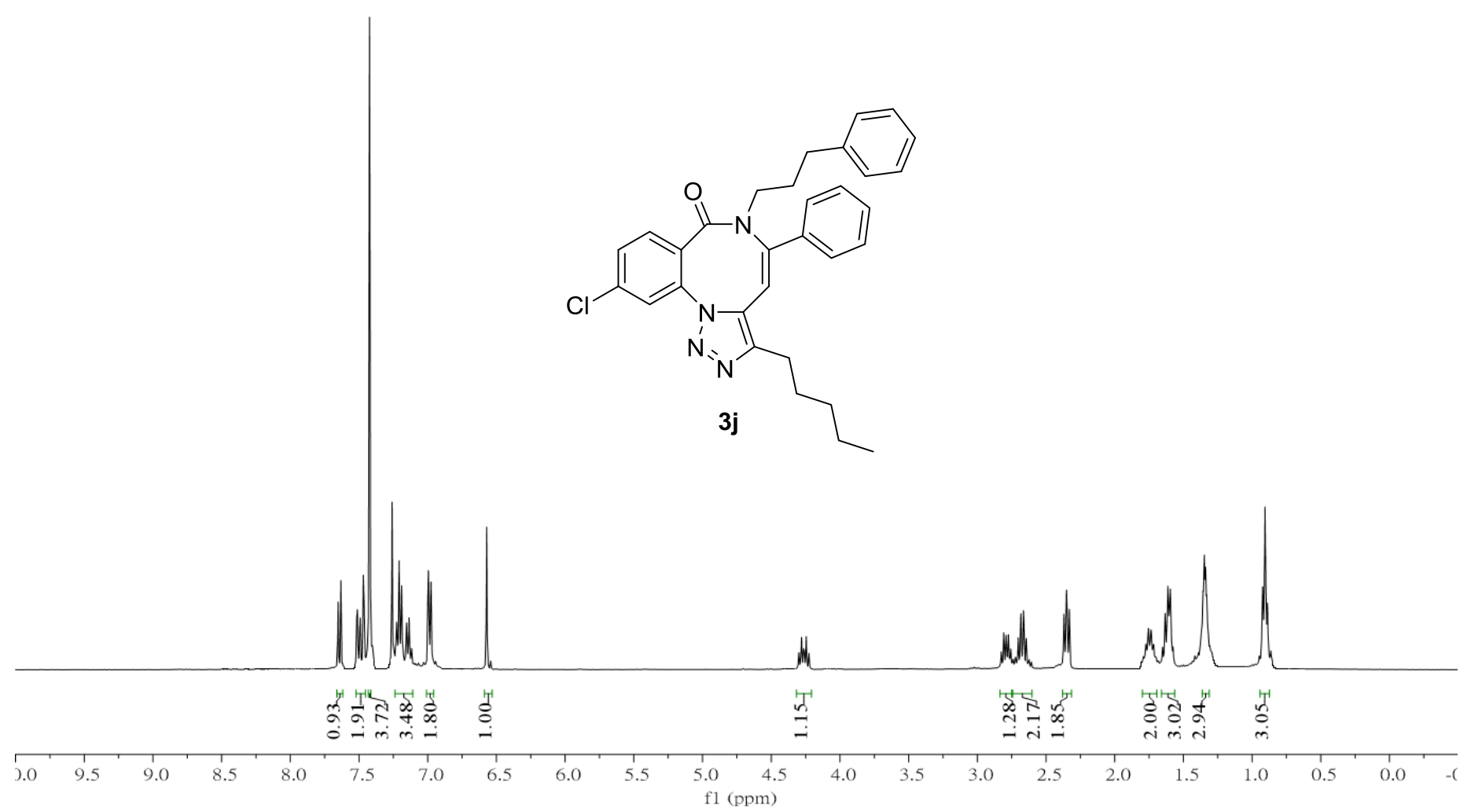

${ }^{1} \mathrm{H}$ NMR Spectrum (400 MHz) of compound $\mathbf{3 j}$ in $\mathrm{CDCl}_{3}$ 

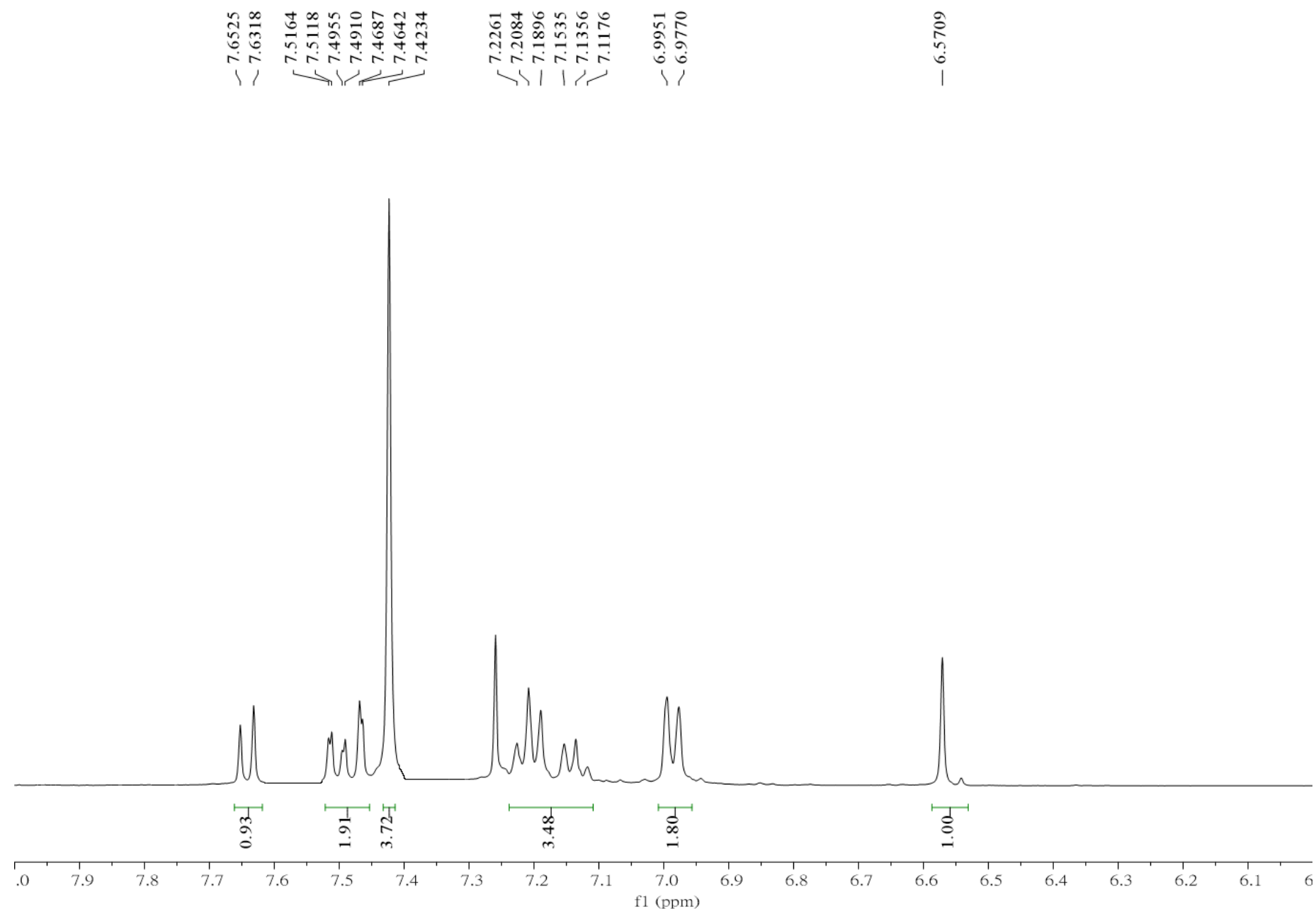

Expansion of ${ }^{1} \mathrm{H}$ NMR Spectrum (400 MHz) of compound $\mathbf{3 j}$ in $\mathrm{CDCl}_{3}$ 

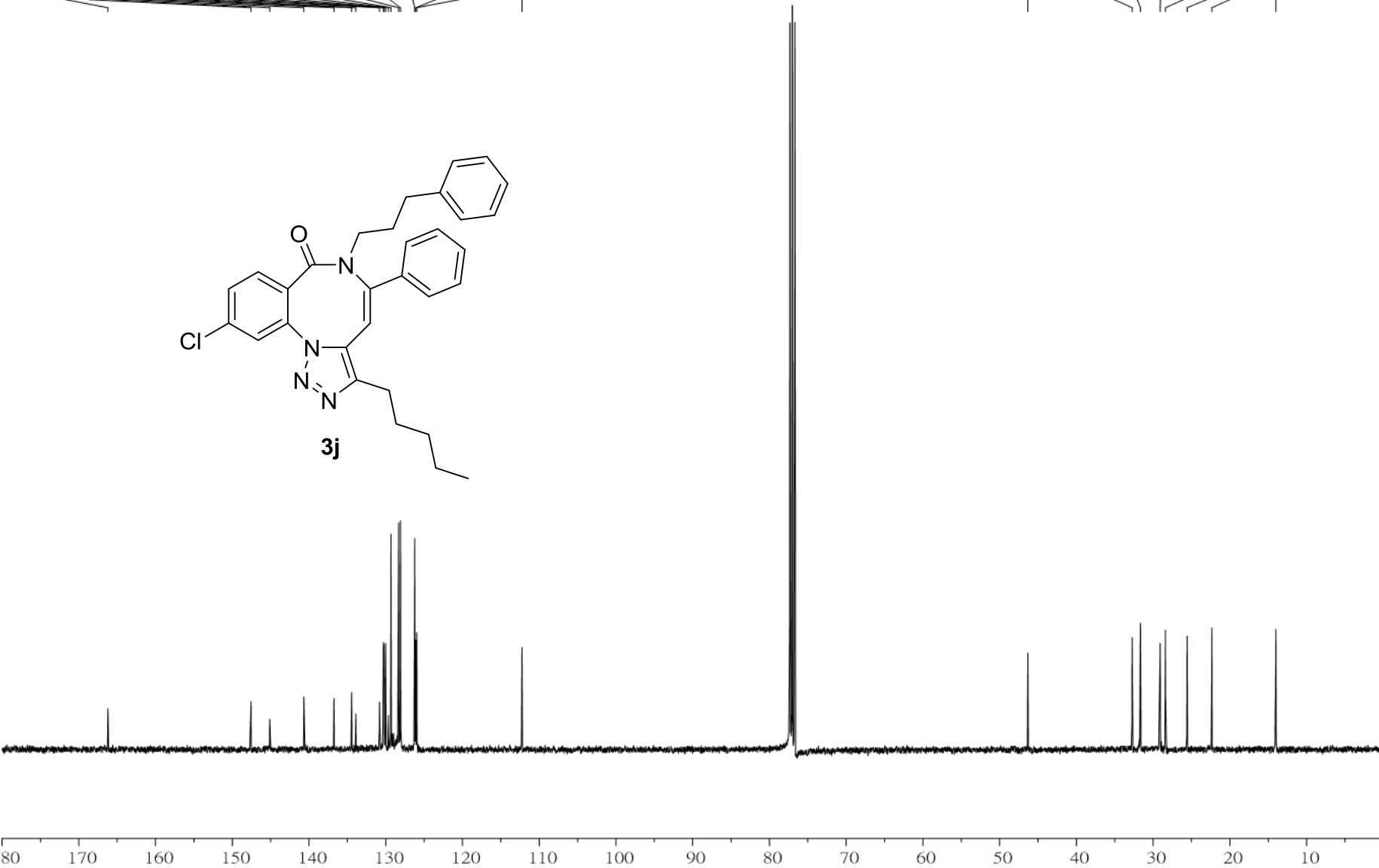

f1 (ppm)

${ }^{13} \mathrm{C}$ NMR Spectrum (101 MHz) of compound 3j in $\mathrm{CDCl}_{3}$ 


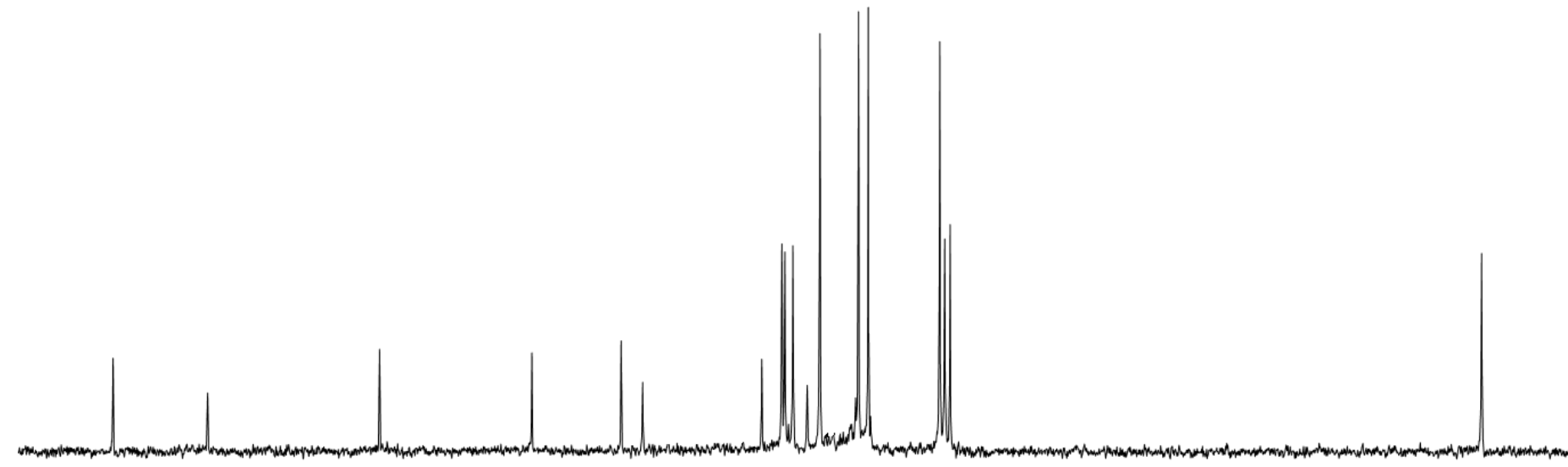

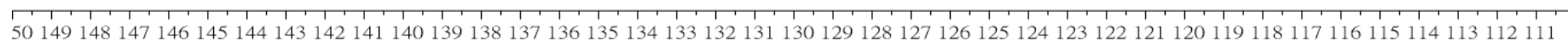
f1 (ppm)

Expansion of ${ }^{13} \mathrm{C}$ NMR Spectrum $(101 \mathrm{MHz})$ of compound $\mathbf{3 j}$ in $\mathrm{CDCl}_{3}$ 


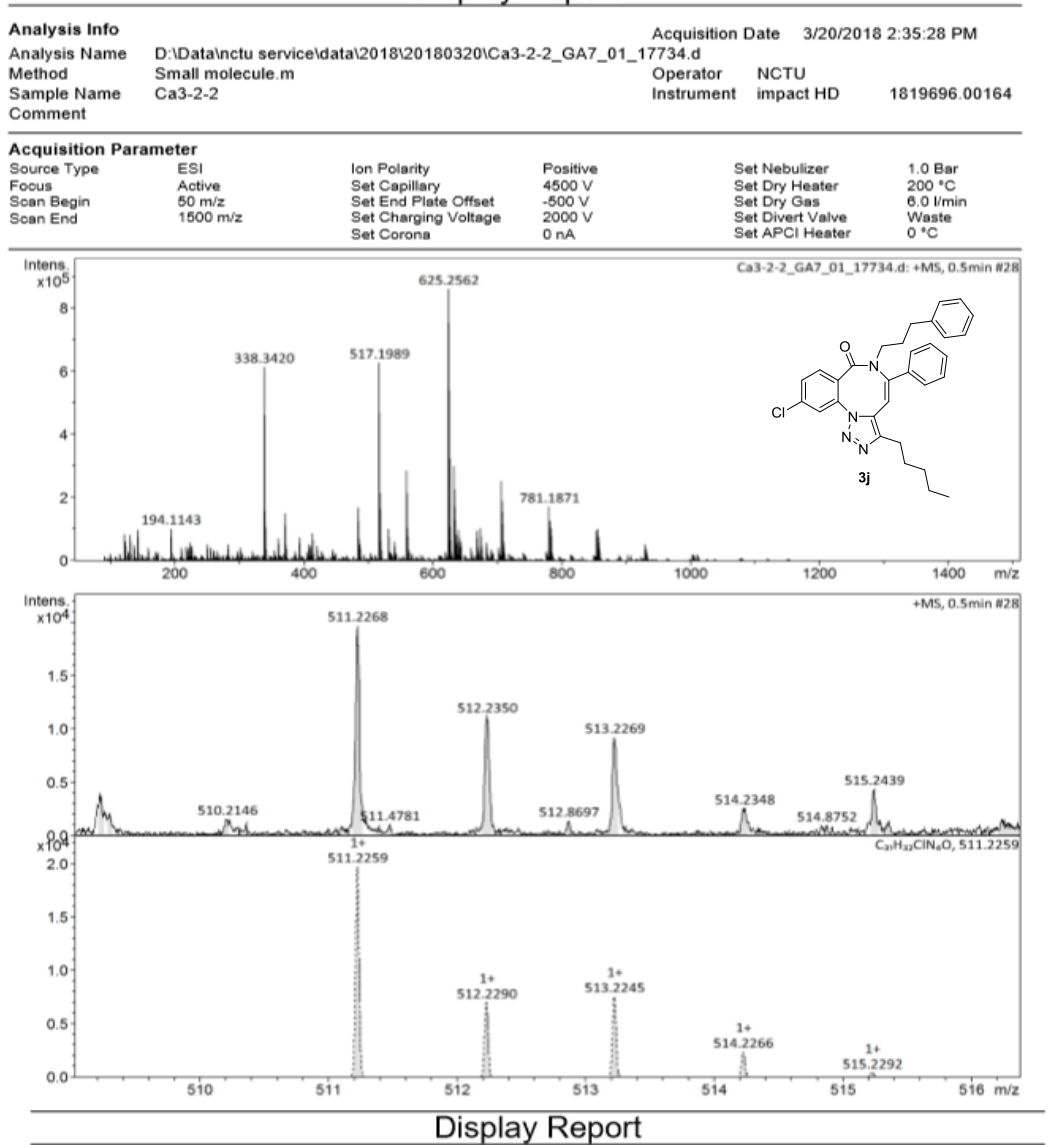

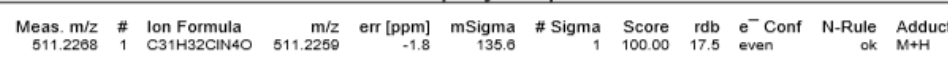

\section{HRMS of compound $\mathbf{3 j}$}




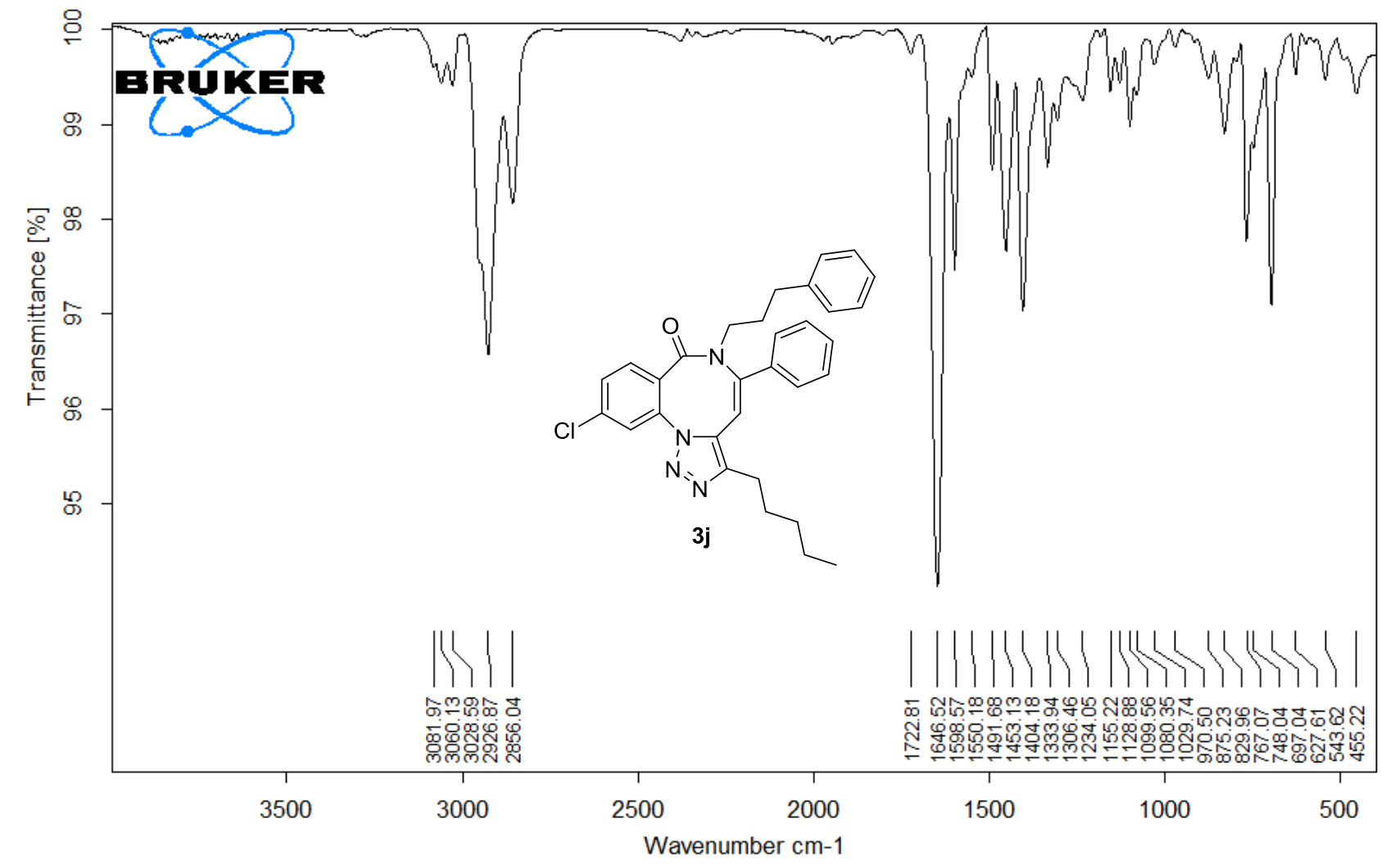

\begin{tabular}{|lcc|c|}
\hline D:IFTIR FILESI201808120180821MIR_TR_DTGS_26137.0 $\quad$ MIR_TR_DTGS_26137 $\quad$ Instrument type and / or accessory & $8 / 21 / 2018$ \\
\hline
\end{tabular}

Page 1/1

IR of compound $\mathbf{3 j}$ 


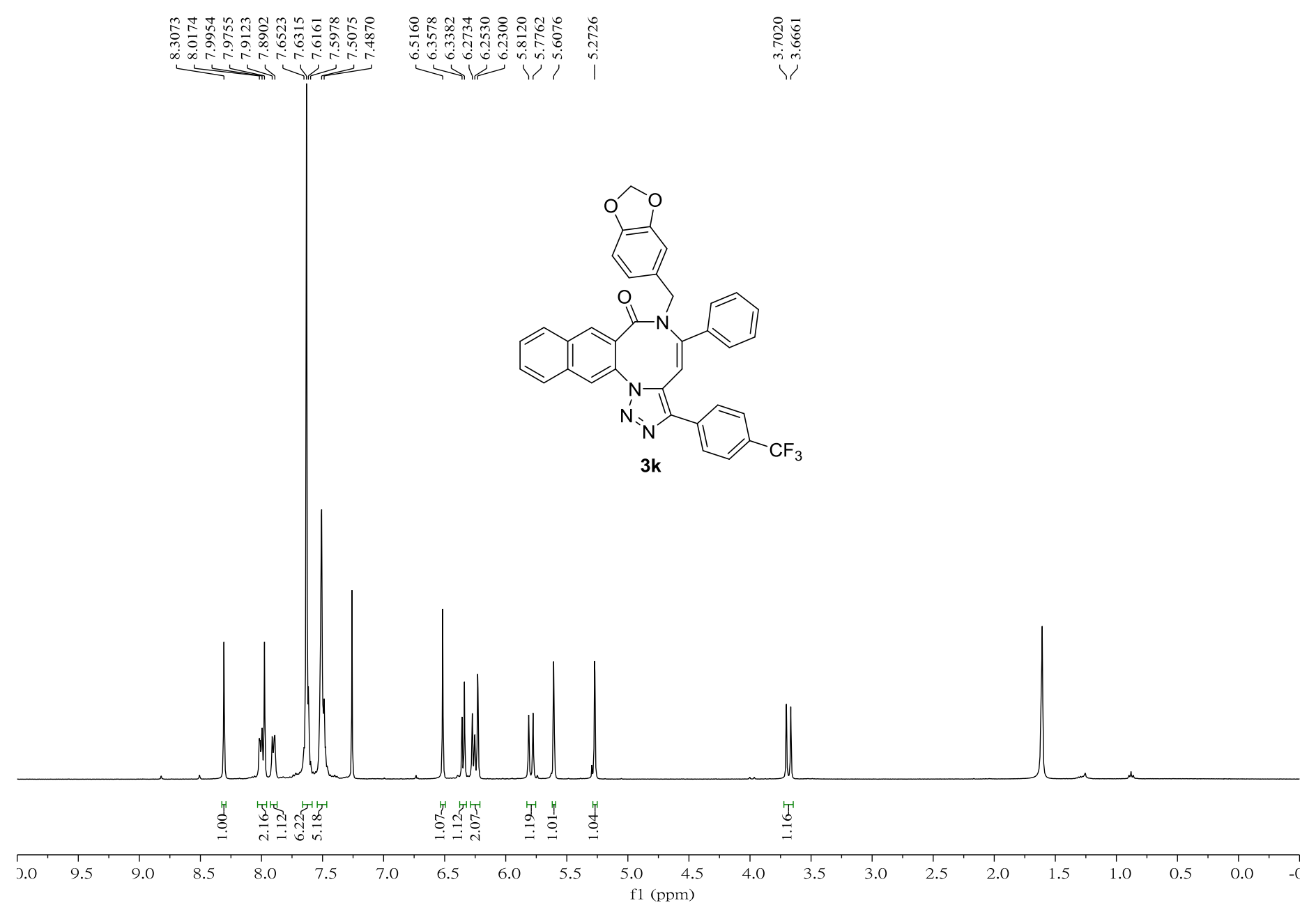

${ }^{1} \mathrm{H}$ NMR Spectrum (400 MHz) of compound 3k in $\mathrm{CDCl}_{3}$ 


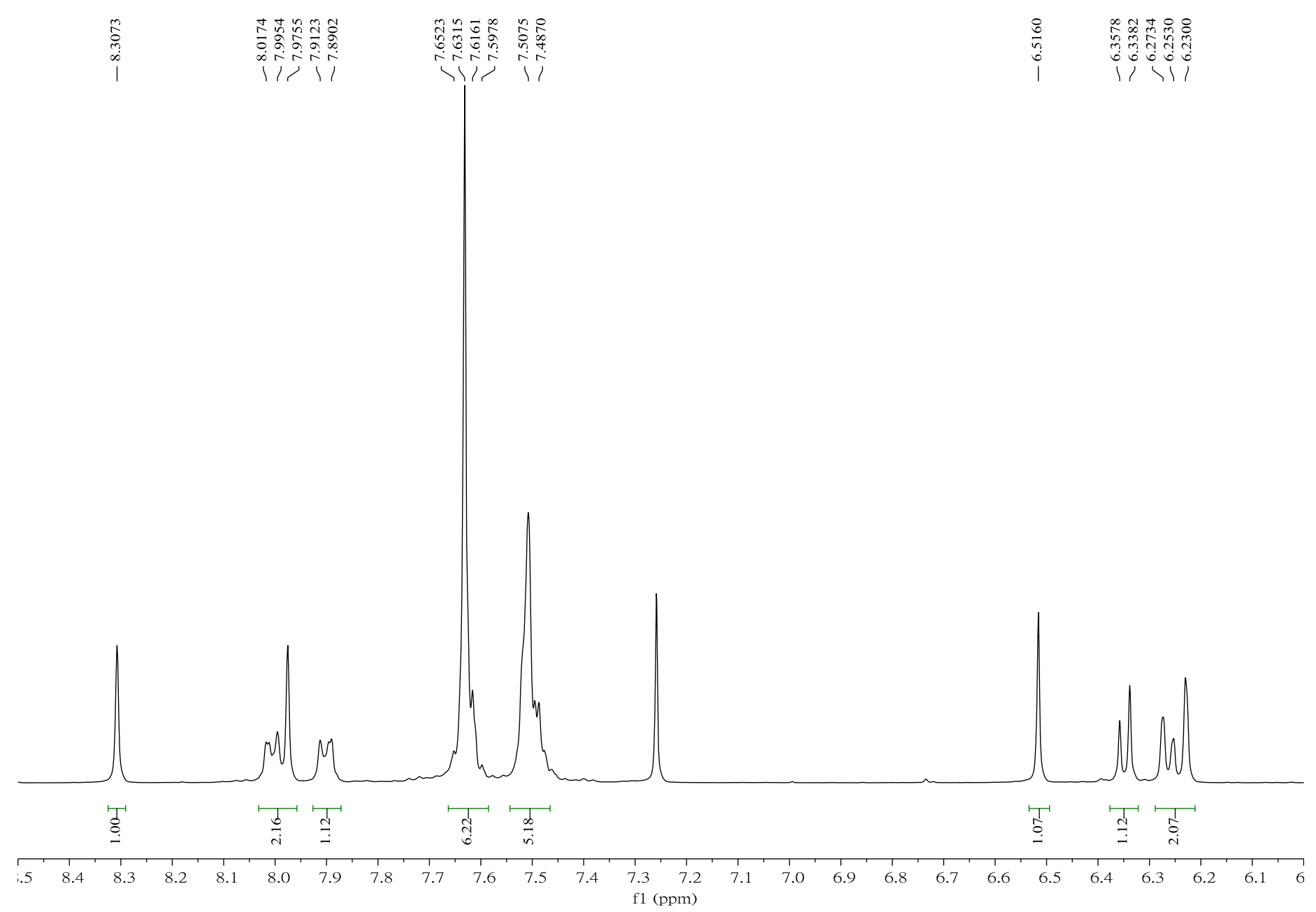

Expansion of ${ }^{1} \mathrm{H}$ NMR Spectrum (400 MHz) of compound 3k in $\mathrm{CDCl}_{3}$ 


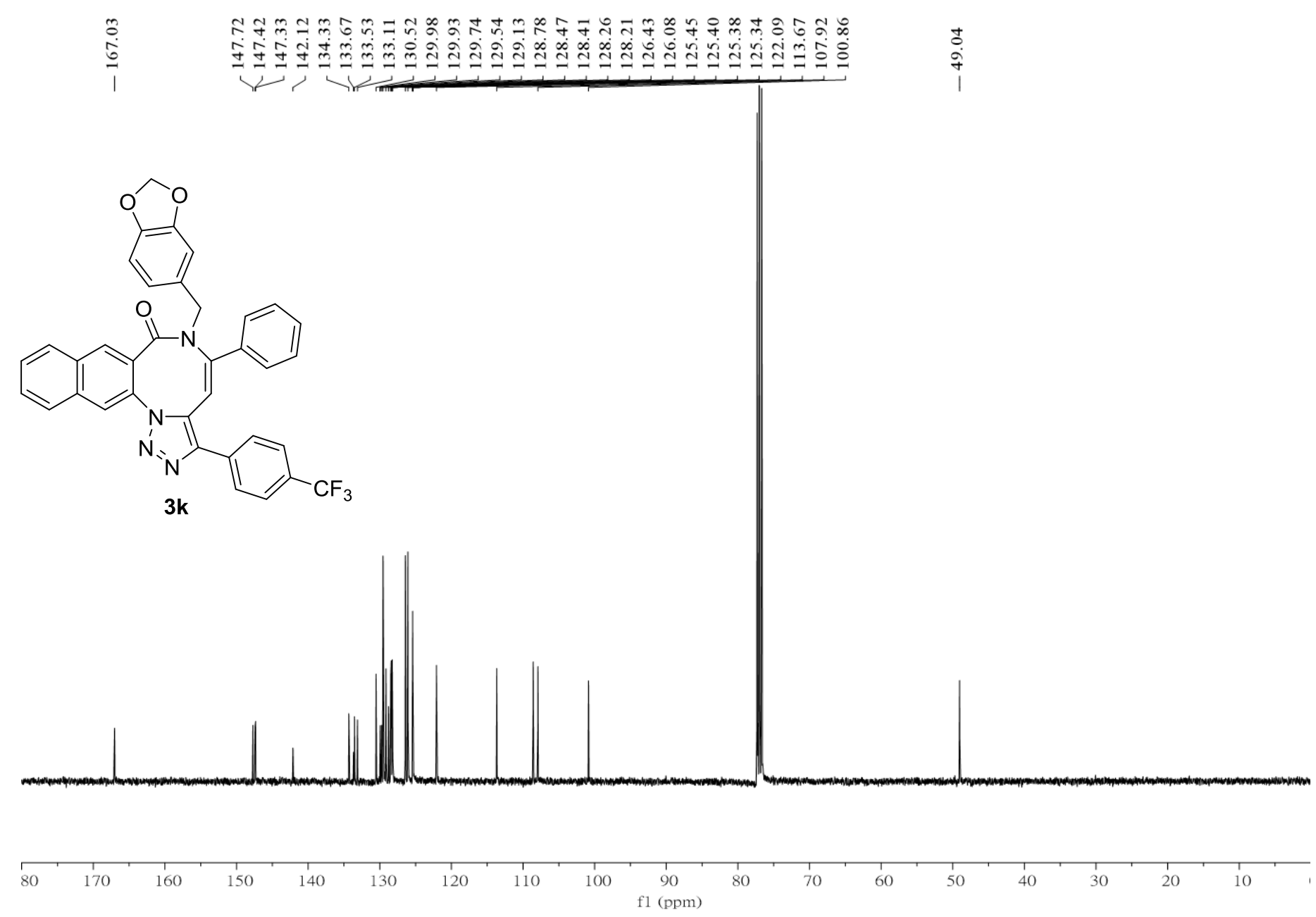

${ }^{13} \mathrm{C}$ NMR Spectrum (101 MHz) of compound $\mathbf{3 k}$ in $\mathrm{CDCl}_{3}$ 

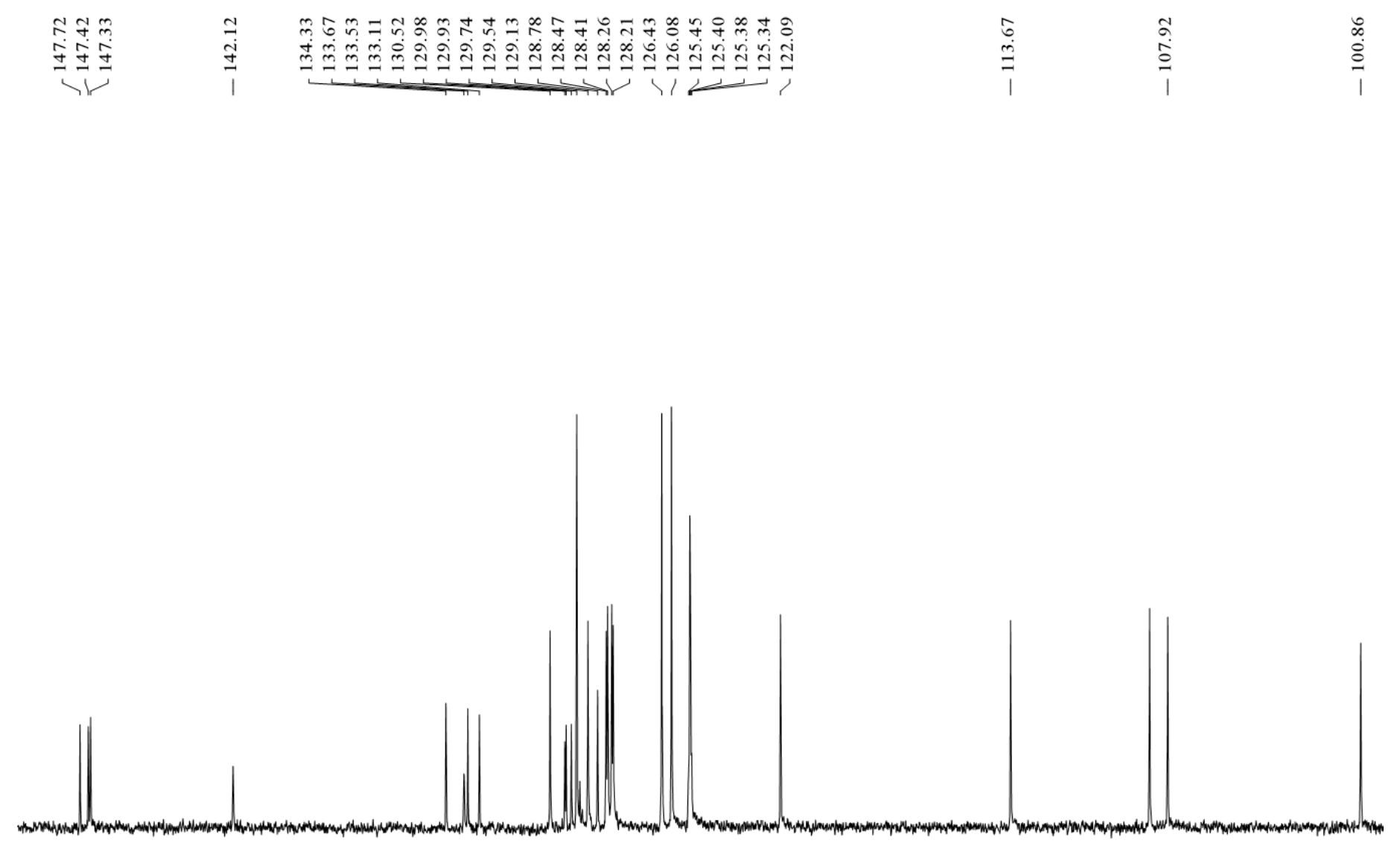

50 145 140 135 130 125
f1 $(\mathrm{ppm})$ 120 115

Expansion of ${ }^{13} \mathrm{C}$ NMR Spectrum $(101 \mathrm{MHz})$ of compound $\mathbf{3 k}$ in $\mathrm{CDCl}_{3}$ 
Display Report

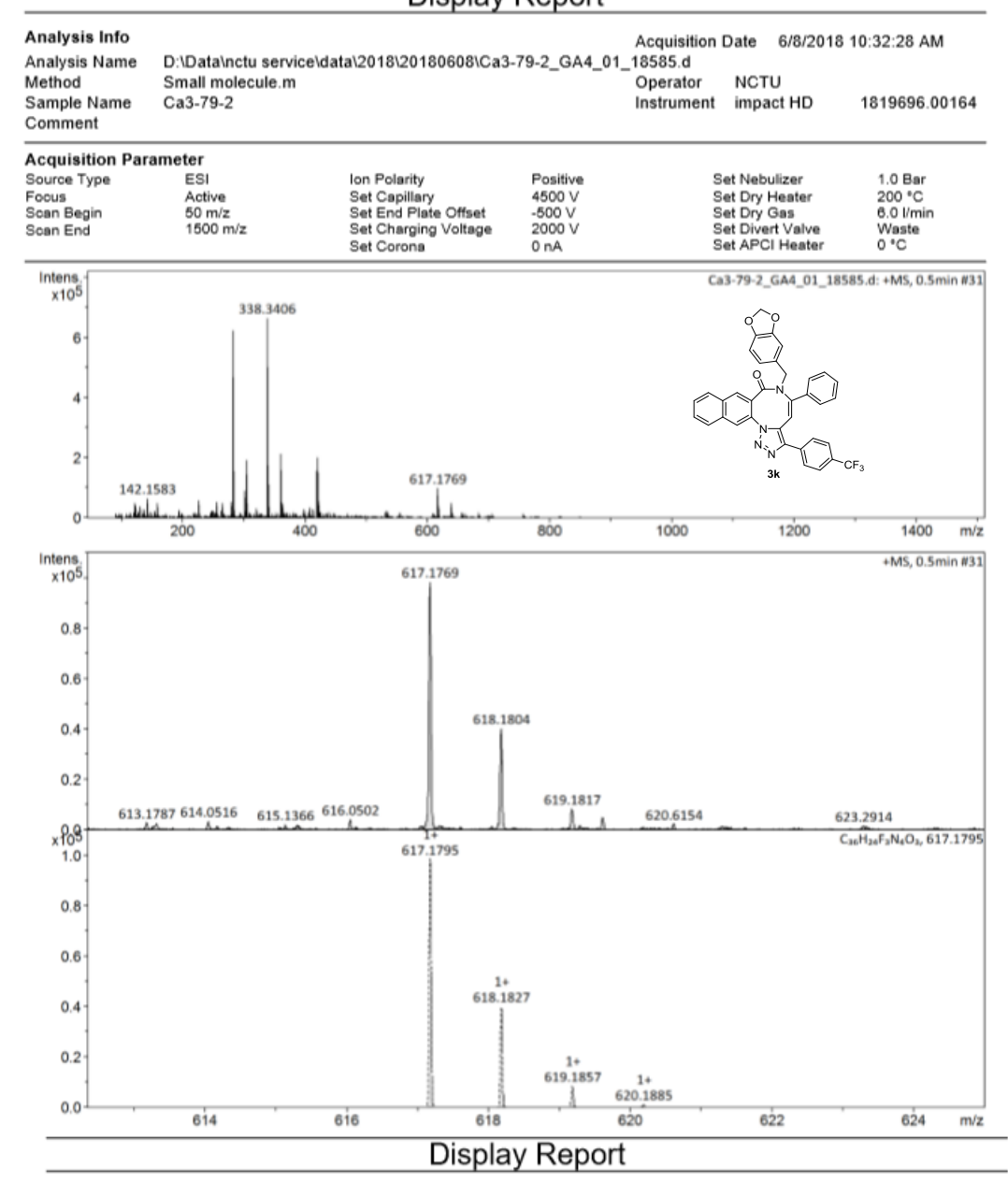

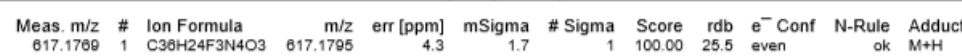

HRMS of compound $\mathbf{3 k}$ 


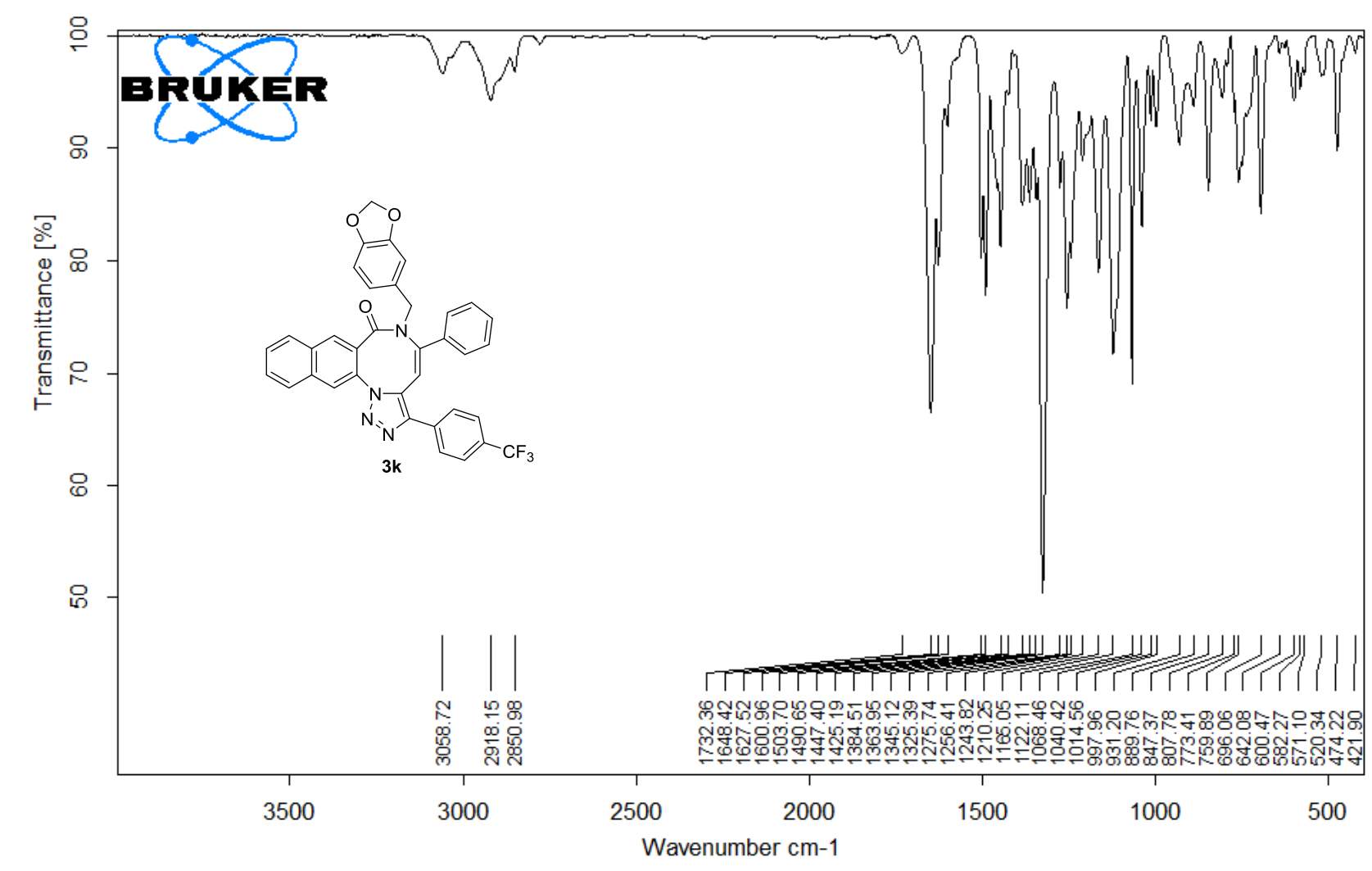

D:IFTIR FILESL201808120180821IMIR_TR_DTGS_26138.0

MIR_TR_DTGS_26138

Instrument type and / or accessory

$8 / 21 / 2018$

Page 1/1

IR of compound $3 \mathbf{k}$ 


\section{X-ray crystallographic data of compound 2a}
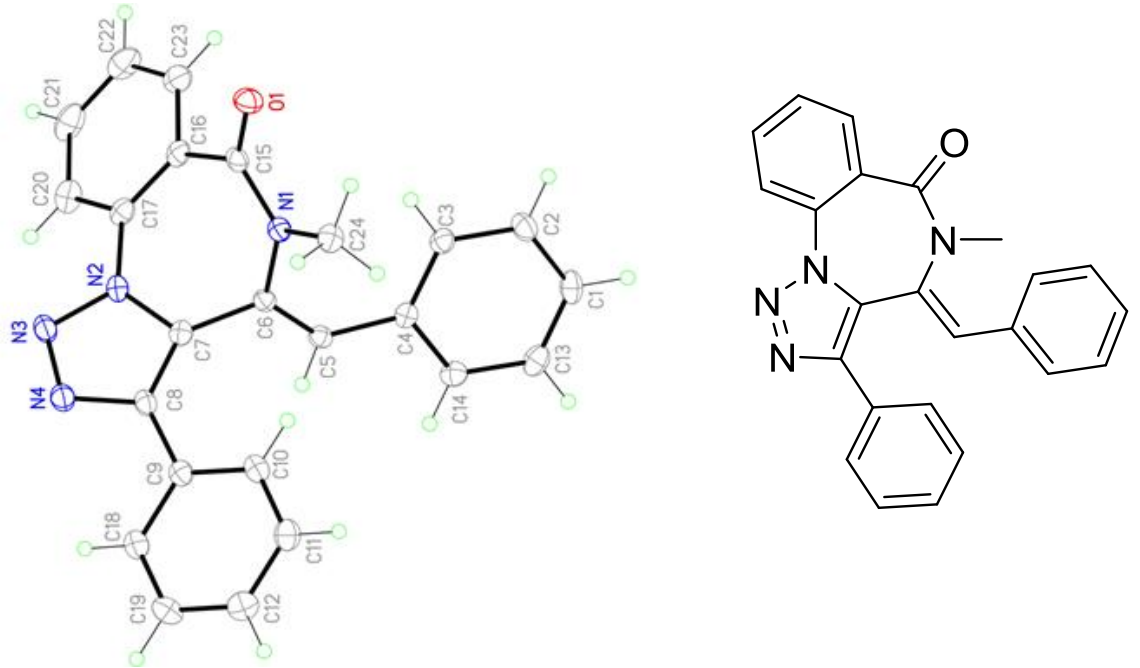

ORTEP diagram of compound 2a. Atomic displacement ellipsoids are drawn at the $50 \%$ probability level

CCDC No.: 1953172

Table S2. Crystal data and structure refinement for $\mathbf{2 a}$

Identification code

Empirical formula

Formula weight

Temperature

Wavelength

Crystal system

Space group

Unit cell dimensions

Volume

$\mathrm{Z}$

Density (calculated)

Absorption coefficient

$\mathrm{F}(000)$

Crystal size

Theta range for data collection
mo_171132LT_0m

C24 H18 N4 O

378.42

$100(2) \mathrm{K}$

$0.71073 \AA$

Monoclinic

P 21/c

$\mathrm{a}=9.7334(14) \AA$

$\mathrm{a}=90^{\circ}$.

$\mathrm{b}=14.458(2) \AA$

$\mathrm{b}=102.605(3)^{\circ}$.

$c=14.073(2) \AA$

$\mathrm{g}=90^{\circ}$.

$1932.8(5) \AA^{3}$

4

$1.300 \mathrm{Mg} / \mathrm{m}^{3}$

$0.082 \mathrm{~mm}^{-1}$

792

$0.25 \times 0.20 \times 0.15 \mathrm{~mm}^{3}$

2.045 to $26.529^{\circ}$. 
Index ranges

Reflections collected

Independent reflections

Completeness to theta $=25.242^{\circ}$

Absorption correction

Max. and min. transmission

Refinement method

Data / restraints / parameters

Goodness-of-fit on $\mathrm{F}^{2}$

Final $\mathrm{R}$ indices [I $>2 \operatorname{sigma}(\mathrm{I})]$

$\mathrm{R}$ indices (all data)

Extinction coefficient

Largest diff. peak and hole $-12<=\mathrm{h}<=12,-18<=\mathrm{k}<=18,-17<=\mathrm{l}<=17$

14050

$3973[\mathrm{R}(\mathrm{int})=0.0360]$

$99.5 \%$

Semi-empirical from equivalents

0.9485 and 0.8519

Full-matrix least-squares on $\mathrm{F}^{2}$

3973 / 0 / 263

1.027

$\mathrm{R} 1=0.0404, \mathrm{wR} 2=0.0959$

$\mathrm{R} 1=0.0564, \mathrm{wR} 2=0.1052$

$\mathrm{n} / \mathrm{a}$

0.271 and -0.228 e. $\AA^{-3}$

Table S3. Atomic coordinates (x 10 $)$ and equivalent isotropic displacement parameters $\left(\AA^{2} \times 10^{3}\right)$ for mo_171132lt_0m. U(eq) is defined as one third of the trace of the orthogonalized $\mathrm{U}^{\mathrm{ij}}$ tensor.

\begin{tabular}{|c|c|c|c|c|}
\hline & $\mathrm{x}$ & $\mathrm{y}$ & $\mathrm{z}$ & $\mathrm{U}(\mathrm{eq})$ \\
\hline $\mathrm{O}(1)$ & $5741(1)$ & 2581(1) & 1894(1) & $23(1)$ \\
\hline $\mathrm{N}(1)$ & $4606(1)$ & $1256(1)$ & 2111(1) & $16(1)$ \\
\hline $\mathrm{N}(2)$ & $6461(1)$ & $-189(1)$ & $1442(1)$ & $17(1)$ \\
\hline $\mathrm{N}(3)$ & $6744(1)$ & $-773(1)$ & $749(1)$ & $20(1)$ \\
\hline $\mathrm{N}(4)$ & $5569(1)$ & $-1214(1)$ & $385(1)$ & $20(1)$ \\
\hline $\mathrm{C}(1)$ & $2678(2)$ & $886(1)$ & $5436(1)$ & $24(1)$ \\
\hline $\mathrm{C}(2)$ & $3315(2)$ & $1506(1)$ & 4908(1) & $22(1)$ \\
\hline $\mathrm{C}(3)$ & $3830(1)$ & 1199(1) & $4115(1)$ & $18(1)$ \\
\hline $\mathrm{C}(4)$ & $3717(1)$ & $266(1)$ & $3842(1)$ & $15(1)$ \\
\hline $\mathrm{C}(5)$ & $4213(1)$ & $-135(1)$ & $3016(1)$ & $15(1)$ \\
\hline$C(6)$ & $4620(1)$ & $276(1)$ & $2272(1)$ & $15(1)$ \\
\hline$C(7)$ & $5099(1)$ & $-275(1)$ & $1525(1)$ & $16(1)$ \\
\hline $\mathrm{C}(8)$ & $4525(2)$ & $-927(1)$ & $837(1)$ & $17(1)$ \\
\hline $\mathrm{C}(9)$ & $3076(1)$ & $-1291(1)$ & $536(1)$ & $16(1)$ \\
\hline$C(10)$ & 1919(2) & $-806(1)$ & $726(1)$ & $21(1)$ \\
\hline $\mathrm{C}(11)$ & $559(2)$ & $-1143(1)$ & $400(1)$ & $22(1)$ \\
\hline$C(12)$ & $333(2)$ & $-1963(1)$ & $-123(1)$ & $23(1)$ \\
\hline$C(13)$ & $2575(2)$ & $-41(1)$ & $5184(1)$ & $23(1)$ \\
\hline$C(14)$ & $3110(2)$ & $-350(1)$ & $4401(1)$ & $18(1)$ \\
\hline
\end{tabular}




$\begin{array}{lrrrr}\mathrm{C}(15) & 5788(1) & 1750(1) & 2074(1) & 17(1) \\ \mathrm{C}(16) & 7198(1) & 1266(1) & 2319(1) & 18(1) \\ \mathrm{C}(17) & 7517(1) & 385(1) & 2015(1) & 18(1) \\ \mathrm{C}(18) & 2834(2) & -2113(1) & 5(1) & 20(1) \\ \mathrm{C}(19) & 1469(2) & -2443(1) & -322(1) & 24(1) \\ \mathrm{C}(20) & 8890(2) & 40(1) & 2254(1) & 24(1) \\ \mathrm{C}(21) & 9955(2) & 579(1) & 2794(1) & 29(1) \\ \mathrm{C}(22) & 9668(2) & 1454(1) & 3098(1) & 29(1) \\ \mathrm{C}(23) & 8306(2) & 1793(1) & 2859(1) & 23(1) \\ \mathrm{C}(24) & 3227(2) & 1701(1) & 1783(1) & 20(1)\end{array}$


Table S4. Bond lengths $[\AA]$ and angles $\left[{ }^{\circ}\right]$ for mo_171132lt_0m.

\begin{tabular}{|c|c|}
\hline $\mathrm{O}(1)-\mathrm{C}(15)$ & $1.2265(17)$ \\
\hline $\mathrm{N}(1)-\mathrm{C}(15)$ & $1.3650(18)$ \\
\hline $\mathrm{N}(1)-\mathrm{C}(6)$ & $1.4349(18)$ \\
\hline $\mathrm{N}(1)-\mathrm{C}(24)$ & $1.4692(17)$ \\
\hline $\mathrm{N}(2)-\mathrm{C}(7)$ & $1.3612(17)$ \\
\hline $\mathrm{N}(2)-\mathrm{N}(3)$ & $1.3626(17)$ \\
\hline $\mathrm{N}(2)-\mathrm{C}(17)$ & $1.4261(19)$ \\
\hline $\mathrm{N}(3)-\mathrm{N}(4)$ & $1.3128(17)$ \\
\hline $\mathrm{N}(4)-\mathrm{C}(8)$ & $1.3764(18)$ \\
\hline $\mathrm{C}(1)-\mathrm{C}(13)$ & $1.385(2)$ \\
\hline $\mathrm{C}(1)-\mathrm{C}(2)$ & $1.394(2)$ \\
\hline $\mathrm{C}(1)-\mathrm{H}(1)$ & 0.9500 \\
\hline $\mathrm{C}(2)-\mathrm{C}(3)$ & $1.391(2)$ \\
\hline $\mathrm{C}(2)-\mathrm{H}(4)$ & 0.9500 \\
\hline $\mathrm{C}(3)-\mathrm{C}(4)$ & $1.401(2)$ \\
\hline $\mathrm{C}(3)-\mathrm{H}(5)$ & 0.9500 \\
\hline $\mathrm{C}(4)-\mathrm{C}(14)$ & $1.4021(19)$ \\
\hline $\mathrm{C}(4)-\mathrm{C}(5)$ & $1.4707(19)$ \\
\hline$C(5)-C(6)$ & $1.337(2)$ \\
\hline $\mathrm{C}(5)-\mathrm{H}(6)$ & 0.9500 \\
\hline $\mathrm{C}(6)-\mathrm{C}(7)$ & $1.4740(19)$ \\
\hline $\mathrm{C}(7)-\mathrm{C}(8)$ & $1.379(2)$ \\
\hline $\mathrm{C}(8)-\mathrm{C}(9)$ & $1.478(2)$ \\
\hline $\mathrm{C}(9)-\mathrm{C}(18)$ & $1.397(2)$ \\
\hline C(9)-C(10) & $1.3997(19)$ \\
\hline $\mathrm{C}(10)-\mathrm{C}(11)$ & $1.391(2)$ \\
\hline $\mathrm{C}(10)-\mathrm{H}(8)$ & 0.9500 \\
\hline $\mathrm{C}(11)-\mathrm{C}(12)$ & $1.387(2)$ \\
\hline $\mathrm{C}(11)-\mathrm{H}(9)$ & 0.9500 \\
\hline $\mathrm{C}(12)-\mathrm{C}(19)$ & $1.385(2)$ \\
\hline $\mathrm{C}(12)-\mathrm{H}(2)$ & 0.9500 \\
\hline $\mathrm{C}(13)-\mathrm{C}(14)$ & 1.391(2) \\
\hline $\mathrm{C}(13)-\mathrm{H}(3)$ & 0.9500 \\
\hline $\mathrm{C}(14)-\mathrm{H}(18)$ & 0.9500 \\
\hline$C(15)-C(16)$ & $1.5118(19)$ \\
\hline $\mathrm{C}(16)-\mathrm{C}(17)$ & $1.400(2)$ \\
\hline$C(16)-C(23)$ & $1.401(2)$ \\
\hline $\mathrm{C}(17)-\mathrm{C}(20)$ & $1.397(2)$ \\
\hline $\mathrm{C}(18)-\mathrm{C}(19)$ & $1.392(2)$ \\
\hline
\end{tabular}




\begin{tabular}{|c|c|}
\hline $\mathrm{C}(18)-\mathrm{H}(7)$ & 0.9500 \\
\hline $\mathrm{C}(19)-\mathrm{H}(10)$ & 0.9500 \\
\hline$C(20)-C(21)$ & $1.384(2)$ \\
\hline $\mathrm{C}(20)-\mathrm{H}(14)$ & 0.9500 \\
\hline$C(21)-C(22)$ & $1.384(3)$ \\
\hline $\mathrm{C}(21)-\mathrm{H}(13)$ & 0.9500 \\
\hline $\mathrm{C}(22)-\mathrm{C}(23)$ & $1.384(2)$ \\
\hline $\mathrm{C}(22)-\mathrm{H}(12)$ & 0.9500 \\
\hline $\mathrm{C}(23)-\mathrm{H}(11)$ & 0.9500 \\
\hline $\mathrm{C}(24)-\mathrm{H}(16)$ & 0.9800 \\
\hline $\mathrm{C}(24)-\mathrm{H}(15)$ & 0.9800 \\
\hline $\mathrm{C}(24)-\mathrm{H}(17)$ & 0.9800 \\
\hline $\mathrm{C}(15)-\mathrm{N}(1)-\mathrm{C}(6)$ & $122.93(11)$ \\
\hline $\mathrm{C}(15)-\mathrm{N}(1)-\mathrm{C}(24)$ & $118.61(12)$ \\
\hline $\mathrm{C}(6)-\mathrm{N}(1)-\mathrm{C}(24)$ & $117.33(11)$ \\
\hline $\mathrm{C}(7)-\mathrm{N}(2)-\mathrm{N}(3)$ & $110.99(12)$ \\
\hline $\mathrm{C}(7)-\mathrm{N}(2)-\mathrm{C}(17)$ & $126.81(12)$ \\
\hline $\mathrm{N}(3)-\mathrm{N}(2)-\mathrm{C}(17)$ & $122.13(11)$ \\
\hline $\mathrm{N}(4)-\mathrm{N}(3)-\mathrm{N}(2)$ & $106.58(11)$ \\
\hline $\mathrm{N}(3)-\mathrm{N}(4)-\mathrm{C}(8)$ & 109.94(12) \\
\hline$C(13)-C(1)-C(2)$ & $120.07(14)$ \\
\hline $\mathrm{C}(13)-\mathrm{C}(1)-\mathrm{H}(1)$ & 120.0 \\
\hline $\mathrm{C}(2)-\mathrm{C}(1)-\mathrm{H}(1)$ & 120.0 \\
\hline$C(3)-C(2)-C(1)$ & $120.19(14)$ \\
\hline $\mathrm{C}(3)-\mathrm{C}(2)-\mathrm{H}(4)$ & 119.9 \\
\hline $\mathrm{C}(1)-\mathrm{C}(2)-\mathrm{H}(4)$ & 119.9 \\
\hline$C(2)-C(3)-C(4)$ & $120.50(13)$ \\
\hline $\mathrm{C}(2)-\mathrm{C}(3)-\mathrm{H}(5)$ & 119.8 \\
\hline $\mathrm{C}(4)-\mathrm{C}(3)-\mathrm{H}(5)$ & 119.8 \\
\hline$C(3)-C(4)-C(14)$ & $118.34(13)$ \\
\hline$C(3)-C(4)-C(5)$ & $125.27(12)$ \\
\hline $\mathrm{C}(14)-\mathrm{C}(4)-\mathrm{C}(5)$ & $116.37(13)$ \\
\hline$C(6)-C(5)-C(4)$ & $130.37(13)$ \\
\hline $\mathrm{C}(6)-\mathrm{C}(5)-\mathrm{H}(6)$ & 114.8 \\
\hline $\mathrm{C}(4)-\mathrm{C}(5)-\mathrm{H}(6)$ & 114.8 \\
\hline $\mathrm{C}(5)-\mathrm{C}(6)-\mathrm{N}(1)$ & $124.66(13)$ \\
\hline$C(5)-C(6)-C(7)$ & $120.85(13)$ \\
\hline $\mathrm{N}(1)-\mathrm{C}(6)-\mathrm{C}(7)$ & $114.47(12)$ \\
\hline $\mathrm{N}(2)-\mathrm{C}(7)-\mathrm{C}(8)$ & $104.79(12)$ \\
\hline$N(2)-C(7)-C(6)$ & $118.56(12)$ \\
\hline$C(8)-C(7)-C(6)$ & $136.62(12)$ \\
\hline
\end{tabular}




\begin{tabular}{|c|c|}
\hline $\mathrm{N}(4)-\mathrm{C}(8)-\mathrm{C}(7)$ & $107.69(12)$ \\
\hline $\mathrm{N}(4)-\mathrm{C}(8)-\mathrm{C}(9)$ & $120.99(13)$ \\
\hline $\mathrm{C}(7)-\mathrm{C}(8)-\mathrm{C}(9)$ & $131.29(13)$ \\
\hline $\mathrm{C}(18)-\mathrm{C}(9)-\mathrm{C}(10)$ & $118.66(13)$ \\
\hline $\mathrm{C}(18)-\mathrm{C}(9)-\mathrm{C}(8)$ & $119.65(12)$ \\
\hline $\mathrm{C}(10)-\mathrm{C}(9)-\mathrm{C}(8)$ & $121.60(13)$ \\
\hline $\mathrm{C}(11)-\mathrm{C}(10)-\mathrm{C}(9)$ & $120.59(14)$ \\
\hline $\mathrm{C}(11)-\mathrm{C}(10)-\mathrm{H}(8)$ & 119.7 \\
\hline $\mathrm{C}(9)-\mathrm{C}(10)-\mathrm{H}(8)$ & 119.7 \\
\hline $\mathrm{C}(12)-\mathrm{C}(11)-\mathrm{C}(10)$ & $120.25(14)$ \\
\hline $\mathrm{C}(12)-\mathrm{C}(11)-\mathrm{H}(9)$ & 119.9 \\
\hline $\mathrm{C}(10)-\mathrm{C}(11)-\mathrm{H}(9)$ & 119.9 \\
\hline $\mathrm{C}(19)-\mathrm{C}(12)-\mathrm{C}(11)$ & $119.60(14)$ \\
\hline $\mathrm{C}(19)-\mathrm{C}(12)-\mathrm{H}(2)$ & 120.2 \\
\hline $\mathrm{C}(11)-\mathrm{C}(12)-\mathrm{H}(2)$ & 120.2 \\
\hline $\mathrm{C}(1)-\mathrm{C}(13)-\mathrm{C}(14)$ & $119.70(14)$ \\
\hline $\mathrm{C}(1)-\mathrm{C}(13)-\mathrm{H}(3)$ & 120.1 \\
\hline $\mathrm{C}(14)-\mathrm{C}(13)-\mathrm{H}(3)$ & 120.1 \\
\hline$C(13)-C(14)-C(4)$ & $121.14(14)$ \\
\hline $\mathrm{C}(13)-\mathrm{C}(14)-\mathrm{H}(18)$ & 119.4 \\
\hline $\mathrm{C}(4)-\mathrm{C}(14)-\mathrm{H}(18)$ & 119.4 \\
\hline $\mathrm{O}(1)-\mathrm{C}(15)-\mathrm{N}(1)$ & $121.76(13)$ \\
\hline $\mathrm{O}(1)-\mathrm{C}(15)-\mathrm{C}(16)$ & $119.44(12)$ \\
\hline $\mathrm{N}(1)-\mathrm{C}(15)-\mathrm{C}(16)$ & $118.70(12)$ \\
\hline$C(17)-C(16)-C(23)$ & $117.72(13)$ \\
\hline$C(17)-C(16)-C(15)$ & $127.00(13)$ \\
\hline $\mathrm{C}(23)-\mathrm{C}(16)-\mathrm{C}(15)$ & $115.15(13)$ \\
\hline$C(20)-C(17)-C(16)$ & $121.02(14)$ \\
\hline $\mathrm{C}(20)-\mathrm{C}(17)-\mathrm{N}(2)$ & $117.50(13)$ \\
\hline $\mathrm{C}(16)-\mathrm{C}(17)-\mathrm{N}(2)$ & $121.47(12)$ \\
\hline $\mathrm{C}(19)-\mathrm{C}(18)-\mathrm{C}(9)$ & $120.36(13)$ \\
\hline $\mathrm{C}(19)-\mathrm{C}(18)-\mathrm{H}(7)$ & 119.8 \\
\hline $\mathrm{C}(9)-\mathrm{C}(18)-\mathrm{H}(7)$ & 119.8 \\
\hline $\mathrm{C}(12)-\mathrm{C}(19)-\mathrm{C}(18)$ & $120.54(14)$ \\
\hline $\mathrm{C}(12)-\mathrm{C}(19)-\mathrm{H}(10)$ & 119.7 \\
\hline C(18)-C(19)-H(10) & 119.7 \\
\hline $\mathrm{C}(21)-\mathrm{C}(20)-\mathrm{C}(17)$ & $119.59(15)$ \\
\hline $\mathrm{C}(21)-\mathrm{C}(20)-\mathrm{H}(14)$ & 120.2 \\
\hline $\mathrm{C}(17)-\mathrm{C}(20)-\mathrm{H}(14)$ & 120.2 \\
\hline $\mathrm{C}(22)-\mathrm{C}(21)-\mathrm{C}(20)$ & $120.44(14)$ \\
\hline $\mathrm{C}(22)-\mathrm{C}(21)-\mathrm{H}(13)$ & 119.8 \\
\hline
\end{tabular}




$\begin{array}{ll}\mathrm{C}(20)-\mathrm{C}(21)-\mathrm{H}(13) & 119.8 \\ \mathrm{C}(21)-\mathrm{C}(22)-\mathrm{C}(23) & 119.76(15) \\ \mathrm{C}(21)-\mathrm{C}(22)-\mathrm{H}(12) & 120.1 \\ \mathrm{C}(23)-\mathrm{C}(22)-\mathrm{H}(12) & 120.1 \\ \mathrm{C}(22)-\mathrm{C}(23)-\mathrm{C}(16) & 121.46(15) \\ \mathrm{C}(22)-\mathrm{C}(23)-\mathrm{H}(11) & 119.3 \\ \mathrm{C}(16)-\mathrm{C}(23)-\mathrm{H}(11) & 119.3 \\ \mathrm{~N}(1)-\mathrm{C}(24)-\mathrm{H}(16) & 109.5 \\ \mathrm{~N}(1)-\mathrm{C}(24)-\mathrm{H}(15) & 109.5 \\ \mathrm{H}(16)-\mathrm{C}(24)-\mathrm{H}(15) & 109.5 \\ \mathrm{~N}(1)-\mathrm{C}(24)-\mathrm{H}(17) & 109.5 \\ \mathrm{H}(16)-\mathrm{C}(24)-\mathrm{H}(17) & 109.5 \\ \mathrm{H}(15)-\mathrm{C}(24)-\mathrm{H}(17) & 109.5\end{array}$

Symmetry transformations used to generate equivalent atoms: 
Table S5. Anisotropic displacement parameters $\left(\AA^{2} \times 10^{3}\right)$ for mo_171132lt_0m. The anisotropic displacement factor exponent takes the form: $-2 p^{2}\left[h^{2} a^{* 2} U^{11}+\ldots+2 h \mathrm{k}^{*} b^{*} U^{12}\right]$

\begin{tabular}{|c|c|c|c|c|c|c|}
\hline & $\mathrm{U}^{11}$ & $\mathrm{U}^{22}$ & $\mathrm{U}^{33}$ & $\mathrm{U}^{23}$ & $\mathrm{U}^{13}$ & $\mathrm{U}^{12}$ \\
\hline $\mathrm{O}(1)$ & 29(1) & $16(1)$ & $24(1)$ & $0(1)$ & $8(1)$ & $-2(1)$ \\
\hline $\mathrm{N}(1)$ & 18(1) & $15(1)$ & $14(1)$ & $1(1)$ & $4(1)$ & 1(1) \\
\hline $\mathrm{N}(2)$ & $17(1)$ & $18(1)$ & $17(1)$ & $-1(1)$ & $6(1)$ & $3(1)$ \\
\hline $\mathrm{N}(3)$ & $22(1)$ & $20(1)$ & $18(1)$ & $-3(1)$ & $8(1)$ & $2(1)$ \\
\hline $\mathrm{N}(4)$ & $22(1)$ & 19(1) & 19(1) & $-1(1)$ & $7(1)$ & 1(1) \\
\hline $\mathrm{C}(1)$ & $27(1)$ & $32(1)$ & $15(1)$ & $-1(1)$ & $9(1)$ & $4(1)$ \\
\hline $\mathrm{C}(2)$ & $28(1)$ & $22(1)$ & $17(1)$ & $-3(1)$ & $6(1)$ & $1(1)$ \\
\hline $\mathrm{C}(3)$ & 19(1) & $20(1)$ & $14(1)$ & $1(1)$ & $4(1)$ & $-2(1)$ \\
\hline C(4) & $13(1)$ & $18(1)$ & $13(1)$ & $2(1)$ & $2(1)$ & $2(1)$ \\
\hline $\mathrm{C}(5)$ & $14(1)$ & $14(1)$ & $16(1)$ & $-1(1)$ & $2(1)$ & $1(1)$ \\
\hline C(6) & $13(1)$ & $15(1)$ & $15(1)$ & $-2(1)$ & $2(1)$ & $0(1)$ \\
\hline $\mathrm{C}(7)$ & $17(1)$ & $16(1)$ & $15(1)$ & $3(1)$ & $4(1)$ & $2(1)$ \\
\hline $\mathrm{C}(8)$ & $21(1)$ & $15(1)$ & $14(1)$ & $1(1)$ & $7(1)$ & $2(1)$ \\
\hline $\mathrm{C}(9)$ & $20(1)$ & $17(1)$ & $12(1)$ & $2(1)$ & $4(1)$ & $1(1)$ \\
\hline $\mathrm{C}(10)$ & $24(1)$ & $20(1)$ & 19(1) & $-2(1)$ & $6(1)$ & $2(1)$ \\
\hline $\mathrm{C}(11)$ & $21(1)$ & $26(1)$ & 21(1) & $2(1)$ & $5(1)$ & $5(1)$ \\
\hline $\mathrm{C}(12)$ & $21(1)$ & $26(1)$ & $22(1)$ & $2(1)$ & $0(1)$ & $0(1)$ \\
\hline $\mathrm{C}(13)$ & $25(1)$ & $28(1)$ & $17(1)$ & $6(1)$ & $8(1)$ & $-1(1)$ \\
\hline $\mathrm{C}(14)$ & $20(1)$ & $18(1)$ & $17(1)$ & $2(1)$ & $3(1)$ & $-1(1)$ \\
\hline $\mathrm{C}(15)$ & $22(1)$ & $18(1)$ & 11(1) & $-2(1)$ & $5(1)$ & $-1(1)$ \\
\hline$C(16)$ & $20(1)$ & $23(1)$ & 11(1) & $2(1)$ & $5(1)$ & $-2(1)$ \\
\hline $\mathrm{C}(17)$ & 18(1) & $23(1)$ & $13(1)$ & $3(1)$ & $4(1)$ & $-1(1)$ \\
\hline $\mathrm{C}(18)$ & $22(1)$ & $20(1)$ & $19(1)$ & $0(1)$ & $6(1)$ & $4(1)$ \\
\hline $\mathrm{C}(19)$ & $27(1)$ & $20(1)$ & $22(1)$ & $-4(1)$ & $1(1)$ & $0(1)$ \\
\hline $\mathrm{C}(20)$ & $21(1)$ & $32(1)$ & $21(1)$ & $3(1)$ & $6(1)$ & $4(1)$ \\
\hline $\mathrm{C}(21)$ & $15(1)$ & $48(1)$ & $25(1)$ & $6(1)$ & $3(1)$ & $2(1)$ \\
\hline $\mathrm{C}(22)$ & $22(1)$ & $44(1)$ & $19(1)$ & $0(1)$ & $1(1)$ & $-10(1)$ \\
\hline $\mathrm{C}(23)$ & $24(1)$ & $28(1)$ & $16(1)$ & $-1(1)$ & $4(1)$ & $-6(1)$ \\
\hline $\mathrm{C}(24)$ & $20(1)$ & 19(1) & $22(1)$ & $4(1)$ & $5(1)$ & $5(1)$ \\
\hline
\end{tabular}


Table S6. Hydrogen coordinates (x $\left.10^{4}\right)$ and isotropic displacement parameters $\left(\AA^{2} \times 10^{3}\right)$ for mo_1711321t_0m.

\begin{tabular}{lrrrl}
\hline & $\mathrm{x}$ & $\mathrm{y}$ & $\mathrm{z}$ & $\mathrm{U}(\mathrm{eq})$ \\
& & & & \\
$\mathrm{H}(1)$ & & & & \\
$\mathrm{H}(4)$ & 3399 & 1100 & 5970 & 28 \\
$\mathrm{H}(5)$ & 4260 & 1625 & 3757 & 26 \\
$\mathrm{H}(6)$ & 4250 & -792 & 3011 & 21 \\
$\mathrm{H}(8)$ & 2065 & -243 & 1081 & 18 \\
$\mathrm{H}(9)$ & -218 & -810 & 535 & 25 \\
$\mathrm{H}(2)$ & -596 & -2194 & -343 & 27 \\
$\mathrm{H}(3)$ & 2142 & -464 & 5544 & 28 \\
$\mathrm{H}(18)$ & 3064 & -990 & 4243 & 27 \\
$\mathrm{H}(7)$ & 3606 & -2449 & -134 & 22 \\
$\mathrm{H}(10)$ & 1315 & -3002 & -684 & 28 \\
$\mathrm{H}(14)$ & 9091 & -561 & 2047 & 29 \\
$\mathrm{H}(13)$ & 10891 & 346 & 2956 & 35 \\
$\mathrm{H}(12)$ & 10402 & 1820 & 3470 & 35 \\
$\mathrm{H}(11)$ & 8118 & 2396 & 3066 & 27 \\
$\mathrm{H}(16)$ & 3321 & 2369 & 1900 & 30 \\
$\mathrm{H}(15)$ & 2561 & 1445 & 2146 & 30 \\
$\mathrm{H}(17)$ & 2878 & 1587 & 1086 & 30 \\
\hline & & & &
\end{tabular}




\section{X-ray crystallographic data of compound 3a}
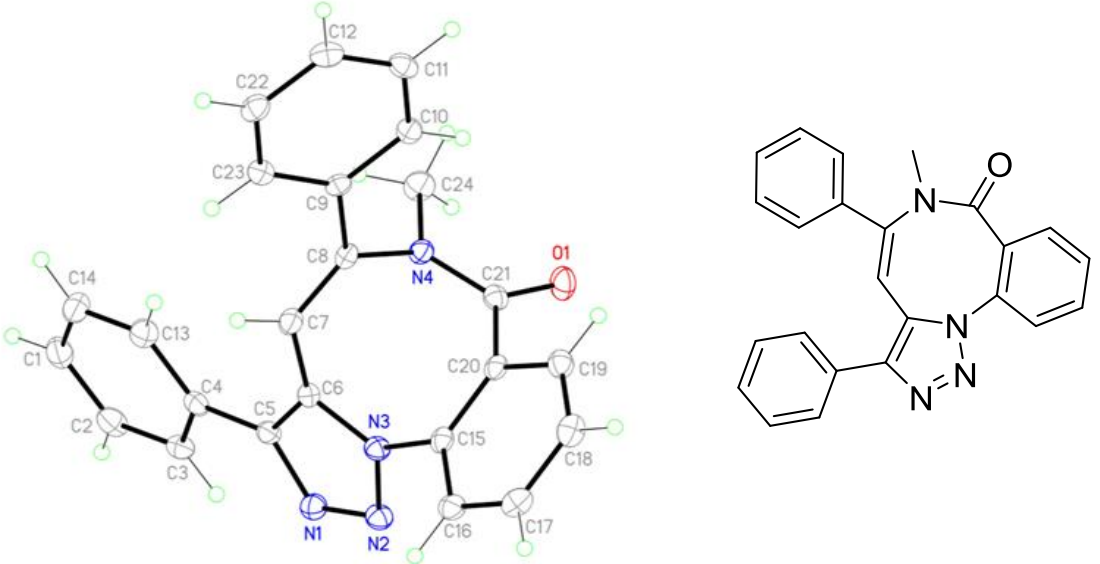

ORTEP diagram of compound 3a. Atomic displacement ellipsoids are drawn at the $50 \%$ probability level

CCDC No. 1953178

Table S7. Crystal data and structure refinement for 3a

Identification code

Empirical formula

Formula weight

Temperature

Wavelength

Crystal system

Space group

Unit cell dimensions

Volume

$\mathrm{Z}$

Density (calculated)

Absorption coefficient

$\mathrm{F}(000)$

Crystal size

Theta range for data collection

Index ranges

Reflections collected

Independent reflections

Completeness to theta $=25.242^{\circ}$
$171133 \mathrm{LT}$

C24 H18 N4 O

378.42

100(2) K

$0.71073 \AA$

Monoclinic

P 21/c

$\mathrm{a}=16.330(3) \AA$

$=90^{\circ}$.

$\mathrm{b}=6.4619(9) \AA$

$=91.333(4)^{\circ}$

$\mathrm{c}=18.194(3) \AA$

$=90^{\circ}$.

1919.4(5) $\AA^{3}$

4

$1.310 \mathrm{Mg} / \mathrm{m}^{3}$

$0.083 \mathrm{~mm}^{-1}$

792

$0.20 \times 0.15 \times 0.13 \mathrm{~mm}^{3}$

1.247 to $25.010^{\circ}$

$-19<=\mathrm{h}<=19,-7<=\mathrm{k}<=7,-21<=\mathrm{l}<=21$

14269

$3389[\mathrm{R}(\mathrm{int})=0.0354]$

$97.7 \%$ 
Absorption correction

Max. and min. transmission

\section{Refinement method}

Data / restraints / parameters

Goodness-of-fit on $\mathrm{F}^{2}$

Final $\mathrm{R}$ indices [I $>2 \operatorname{sigma}(\mathrm{I})]$

$\mathrm{R}$ indices (all data)

Extinction coefficient

Largest diff. peak and hole
Semi-empirical from equivalents

0.9484 and 0.8777

Full-matrix least-squares on $\mathrm{F}^{2}$

3389 / 0 / 263

1.054

$\mathrm{R} 1=0.0384, \mathrm{wR} 2=0.0928$

$\mathrm{R} 1=0.0472, \mathrm{wR} 2=0.0979$

$\mathrm{n} / \mathrm{a}$

0.277 and $-0.242 \mathrm{e} . \AA^{-3}$ 
Table S8. Atomic coordinates (x $\left.10^{4}\right)$ and equivalent isotropic displacement parameters $\left(\AA^{2} \times 10^{3}\right)$ for 171133LT. U(eq) is defined as one third of the trace of the orthogonalized $\mathrm{U}^{\mathrm{ij}}$ tensor.

\begin{tabular}{|c|c|c|c|c|}
\hline & $\mathrm{x}$ & $\mathrm{y}$ & $\mathrm{z}$ & $\mathrm{U}(\mathrm{eq})$ \\
\hline $\mathrm{O}(1)$ & $6324(1)$ & $10978(2)$ & $5728(1)$ & $23(1)$ \\
\hline $\mathrm{N}(1)$ & $9320(1)$ & $12442(2)$ & $4864(1)$ & 18(1) \\
\hline $\mathrm{N}(2)$ & 9092(1) & $11027(2)$ & 5334(1) & $17(1)$ \\
\hline $\mathrm{N}(3)$ & $8490(1)$ & 9918(2) & 4993(1) & $15(1)$ \\
\hline N(4) & $6646(1)$ & $10590(2)$ & $4534(1)$ & $15(1)$ \\
\hline $\mathrm{C}(1)$ & 9197(1) & $16710(3)$ & $2517(1)$ & $24(1)$ \\
\hline C(2) & $9569(1)$ & 16972(2) & $3203(1)$ & $22(1)$ \\
\hline C(3) & $9457(1)$ & $15523(2)$ & $3754(1)$ & 19(1) \\
\hline C(4) & $8975(1)$ & $13764(2)$ & $3622(1)$ & 17(1) \\
\hline C(5) & $8876(1)$ & $12253(2)$ & $4218(1)$ & 16(1) \\
\hline C(6) & $8344(1)$ & $10604(2)$ & $4294(1)$ & $15(1)$ \\
\hline C(7) & 7766(1) & $9480(2)$ & $3812(1)$ & 17(1) \\
\hline C(8) & $6970(1)$ & $9352(2)$ & 3951(1) & $15(1)$ \\
\hline C(9) & $6369(1)$ & $8034(2)$ & $3543(1)$ & $15(1)$ \\
\hline C(10) & $5594(1)$ & $7675(2)$ & $3829(1)$ & 16(1) \\
\hline $\mathrm{C}(11)$ & $5038(1)$ & $6397(2)$ & $3466(1)$ & $18(1)$ \\
\hline $\mathrm{C}(12)$ & $5241(1)$ & $5453(2)$ & 2816(1) & 19(1) \\
\hline $\mathrm{C}(13)$ & $8594(1)$ & $13530(3)$ & 2933(1) & $22(1)$ \\
\hline $\mathrm{C}(14)$ & $8703(1)$ & 14996(3) & $2387(1)$ & $25(1)$ \\
\hline $\mathrm{C}(15)$ & $8100(1)$ & $8253(2)$ & 5372(1) & $15(1)$ \\
\hline $\mathrm{C}(16)$ & $8588(1)$ & $6684(2)$ & 5661(1) & 18(1) \\
\hline C(17) & $8231(1)$ & $5105(2)$ & 6059(1) & 21(1) \\
\hline C(18) & 7392(1) & $5088(2)$ & 6162(1) & 21(1) \\
\hline C(19) & $6910(1)$ & $6678(2)$ & $5874(1)$ & 19(1) \\
\hline $\mathrm{C}(20)$ & $7254(1)$ & $8278(2)$ & $5472(1)$ & $15(1)$ \\
\hline $\mathrm{C}(21)$ & $6707(1)$ & $10062(2)$ & $5253(1)$ & 16(1) \\
\hline $\mathrm{C}(22)$ & $6009(1)$ & $5785(2)$ & 2521(1) & 19(1) \\
\hline$C(23)$ & $6564(1)$ & $7073(2)$ & 2879(1) & $18(1)$ \\
\hline$C(24)$ & $6142(1)$ & $12387(2)$ & $4320(1)$ & $22(1)$ \\
\hline
\end{tabular}


Table S9. Bond lengths $[\AA ̊]$ and angles $\left[{ }^{\circ}\right]$ for $171133 \mathrm{LT}$.

\begin{tabular}{|c|c|}
\hline $\mathrm{O}(1)-\mathrm{C}(21)$ & $1.2295(18)$ \\
\hline $\mathrm{N}(1)-\mathrm{N}(2)$ & $1.3117(18)$ \\
\hline $\mathrm{N}(1)-\mathrm{C}(5)$ & $1.373(2)$ \\
\hline $\mathrm{N}(2)-\mathrm{N}(3)$ & $1.3553(17)$ \\
\hline $\mathrm{N}(3)-\mathrm{C}(6)$ & $1.3631(19)$ \\
\hline $\mathrm{N}(3)-\mathrm{C}(15)$ & $1.4348(19)$ \\
\hline $\mathrm{N}(4)-\mathrm{C}(21)$ & $1.3531(19)$ \\
\hline $\mathrm{N}(4)-\mathrm{C}(8)$ & $1.4401(19)$ \\
\hline $\mathrm{N}(4)-\mathrm{C}(24)$ & $1.4702(19)$ \\
\hline $\mathrm{C}(1)-\mathrm{C}(2)$ & $1.386(2)$ \\
\hline $\mathrm{C}(1)-\mathrm{C}(14)$ & $1.388(2)$ \\
\hline $\mathrm{C}(1)-\mathrm{H}(1)$ & 0.9500 \\
\hline$C(2)-C(3)$ & $1.386(2)$ \\
\hline $\mathrm{C}(2)-\mathrm{H}(18)$ & 0.9500 \\
\hline$C(3)-C(4)$ & $1.401(2)$ \\
\hline $\mathrm{C}(3)-\mathrm{H}(3)$ & 0.9500 \\
\hline $\mathrm{C}(4)-\mathrm{C}(13)$ & $1.394(2)$ \\
\hline$C(4)-C(5)$ & $1.470(2)$ \\
\hline$C(5)-C(6)$ & $1.383(2)$ \\
\hline$C(6)-C(7)$ & $1.466(2)$ \\
\hline$C(7)-C(8)$ & $1.333(2)$ \\
\hline $\mathrm{C}(7)-\mathrm{H}(6)$ & 0.9500 \\
\hline$C(8)-C(9)$ & $1.485(2)$ \\
\hline $\mathrm{C}(9)-\mathrm{C}(10)$ & $1.398(2)$ \\
\hline $\mathrm{C}(9)-\mathrm{C}(23)$ & $1.401(2)$ \\
\hline $\mathrm{C}(10)-\mathrm{C}(11)$ & $1.385(2)$ \\
\hline $\mathrm{C}(10)-\mathrm{H}(7)$ & 0.9500 \\
\hline $\mathrm{C}(11)-\mathrm{C}(12)$ & $1.378(2)$ \\
\hline $\mathrm{C}(11)-\mathrm{H}(10)$ & 0.9500 \\
\hline $\mathrm{C}(12)-\mathrm{C}(22)$ & $1.391(2)$ \\
\hline $\mathrm{C}(12)-\mathrm{H}(2)$ & 0.9500 \\
\hline $\mathrm{C}(13)-\mathrm{C}(14)$ & $1.387(2)$ \\
\hline $\mathrm{C}(13)-\mathrm{H}(4)$ & 0.9500 \\
\hline $\mathrm{C}(14)-\mathrm{H}(5)$ & 0.9500 \\
\hline $\mathrm{C}(15)-\mathrm{C}(16)$ & $1.386(2)$ \\
\hline$C(15)-C(20)$ & $1.398(2)$ \\
\hline $\mathrm{C}(16)-\mathrm{C}(17)$ & $1.387(2)$ \\
\hline $\mathrm{C}(16)-\mathrm{H}(17)$ & 0.9500 \\
\hline $\mathrm{C}(17)-\mathrm{C}(18)$ & $1.388(2)$ \\
\hline
\end{tabular}




\begin{tabular}{|c|c|}
\hline $\mathrm{C}(17)-\mathrm{H}(16)$ & 0.9500 \\
\hline $\mathrm{C}(18)-\mathrm{C}(19)$ & $1.389(2)$ \\
\hline $\mathrm{C}(18)-\mathrm{H}(15)$ & 0.9500 \\
\hline $\mathrm{C}(19)-\mathrm{C}(20)$ & $1.392(2)$ \\
\hline C(19)-H(14) & 0.9500 \\
\hline $\mathrm{C}(20)-\mathrm{C}(21)$ & $1.507(2)$ \\
\hline $\mathrm{C}(22)-\mathrm{C}(23)$ & $1.383(2)$ \\
\hline $\mathrm{C}(22)-\mathrm{H}(9)$ & 0.9500 \\
\hline $\mathrm{C}(23)-\mathrm{H}(8)$ & 0.9500 \\
\hline $\mathrm{C}(24)-\mathrm{H}(13)$ & 0.9800 \\
\hline $\mathrm{C}(24)-\mathrm{H}(12)$ & 0.9800 \\
\hline $\mathrm{C}(24)-\mathrm{H}(11)$ & 0.9800 \\
\hline $\mathrm{N}(2)-\mathrm{N}(1)-\mathrm{C}(5)$ & $110.14(12)$ \\
\hline $\mathrm{N}(1)-\mathrm{N}(2)-\mathrm{N}(3)$ & $106.48(12)$ \\
\hline $\mathrm{N}(2)-\mathrm{N}(3)-\mathrm{C}(6)$ & $111.37(12)$ \\
\hline $\mathrm{N}(2)-\mathrm{N}(3)-\mathrm{C}(15)$ & $120.10(12)$ \\
\hline $\mathrm{C}(6)-\mathrm{N}(3)-\mathrm{C}(15)$ & $128.54(12)$ \\
\hline $\mathrm{C}(21)-\mathrm{N}(4)-\mathrm{C}(8)$ & $123.56(12)$ \\
\hline $\mathrm{C}(21)-\mathrm{N}(4)-\mathrm{C}(24)$ & $118.89(13)$ \\
\hline $\mathrm{C}(8)-\mathrm{N}(4)-\mathrm{C}(24)$ & $117.14(12)$ \\
\hline $\mathrm{C}(2)-\mathrm{C}(1)-\mathrm{C}(14)$ & $119.45(15)$ \\
\hline $\mathrm{C}(2)-\mathrm{C}(1)-\mathrm{H}(1)$ & 120.3 \\
\hline $\mathrm{C}(14)-\mathrm{C}(1)-\mathrm{H}(1)$ & 120.3 \\
\hline$C(1)-C(2)-C(3)$ & $120.45(15)$ \\
\hline $\mathrm{C}(1)-\mathrm{C}(2)-\mathrm{H}(18)$ & 119.8 \\
\hline $\mathrm{C}(3)-\mathrm{C}(2)-\mathrm{H}(18)$ & 119.8 \\
\hline$C(2)-C(3)-C(4)$ & $120.47(15)$ \\
\hline $\mathrm{C}(2)-\mathrm{C}(3)-\mathrm{H}(3)$ & 119.8 \\
\hline $\mathrm{C}(4)-\mathrm{C}(3)-\mathrm{H}(3)$ & 119.8 \\
\hline $\mathrm{C}(13)-\mathrm{C}(4)-\mathrm{C}(3)$ & $118.61(15)$ \\
\hline$C(13)-C(4)-C(5)$ & $122.43(14)$ \\
\hline$C(3)-C(4)-C(5)$ & $118.95(14)$ \\
\hline $\mathrm{N}(1)-\mathrm{C}(5)-\mathrm{C}(6)$ & $107.64(13)$ \\
\hline $\mathrm{N}(1)-\mathrm{C}(5)-\mathrm{C}(4)$ & $120.47(13)$ \\
\hline$C(6)-C(5)-C(4)$ & $131.72(14)$ \\
\hline $\mathrm{N}(3)-\mathrm{C}(6)-\mathrm{C}(5)$ & $104.34(13)$ \\
\hline $\mathrm{N}(3)-\mathrm{C}(6)-\mathrm{C}(7)$ & $119.55(13)$ \\
\hline$C(5)-C(6)-C(7)$ & $135.94(14)$ \\
\hline$C(8)-C(7)-C(6)$ & $122.34(14)$ \\
\hline $\mathrm{C}(8)-\mathrm{C}(7)-\mathrm{H}(6)$ & 118.8 \\
\hline
\end{tabular}




\begin{tabular}{|c|c|}
\hline $\mathrm{C}(6)-\mathrm{C}(7)-\mathrm{H}(6)$ & 118.8 \\
\hline $\mathrm{C}(7)-\mathrm{C}(8)-\mathrm{N}(4)$ & $118.78(13)$ \\
\hline $\mathrm{C}(7)-\mathrm{C}(8)-\mathrm{C}(9)$ & $125.28(14)$ \\
\hline $\mathrm{N}(4)-\mathrm{C}(8)-\mathrm{C}(9)$ & $115.93(13)$ \\
\hline$C(10)-C(9)-C(23)$ & $118.25(14)$ \\
\hline $\mathrm{C}(10)-\mathrm{C}(9)-\mathrm{C}(8)$ & $120.15(14)$ \\
\hline $\mathrm{C}(23)-\mathrm{C}(9)-\mathrm{C}(8)$ & $121.57(14)$ \\
\hline $\mathrm{C}(11)-\mathrm{C}(10)-\mathrm{C}(9)$ & $120.68(15)$ \\
\hline $\mathrm{C}(11)-\mathrm{C}(10)-\mathrm{H}(7)$ & 119.7 \\
\hline $\mathrm{C}(9)-\mathrm{C}(10)-\mathrm{H}(7)$ & 119.7 \\
\hline $\mathrm{C}(12)-\mathrm{C}(11)-\mathrm{C}(10)$ & $120.34(15)$ \\
\hline $\mathrm{C}(12)-\mathrm{C}(11)-\mathrm{H}(10)$ & 119.8 \\
\hline $\mathrm{C}(10)-\mathrm{C}(11)-\mathrm{H}(10)$ & 119.8 \\
\hline $\mathrm{C}(11)-\mathrm{C}(12)-\mathrm{C}(22)$ & $119.99(14)$ \\
\hline $\mathrm{C}(11)-\mathrm{C}(12)-\mathrm{H}(2)$ & 120.0 \\
\hline $\mathrm{C}(22)-\mathrm{C}(12)-\mathrm{H}(2)$ & 120.0 \\
\hline $\mathrm{C}(14)-\mathrm{C}(13)-\mathrm{C}(4)$ & $120.58(15)$ \\
\hline $\mathrm{C}(14)-\mathrm{C}(13)-\mathrm{H}(4)$ & 119.7 \\
\hline $\mathrm{C}(4)-\mathrm{C}(13)-\mathrm{H}(4)$ & 119.7 \\
\hline $\mathrm{C}(13)-\mathrm{C}(14)-\mathrm{C}(1)$ & $120.43(16)$ \\
\hline $\mathrm{C}(13)-\mathrm{C}(14)-\mathrm{H}(5)$ & 119.8 \\
\hline $\mathrm{C}(1)-\mathrm{C}(14)-\mathrm{H}(5)$ & 119.8 \\
\hline$C(16)-C(15)-C(20)$ & $121.32(14)$ \\
\hline $\mathrm{C}(16)-\mathrm{C}(15)-\mathrm{N}(3)$ & $118.30(13)$ \\
\hline $\mathrm{C}(20)-\mathrm{C}(15)-\mathrm{N}(3)$ & $120.32(13)$ \\
\hline$C(15)-C(16)-C(17)$ & $119.47(15)$ \\
\hline $\mathrm{C}(15)-\mathrm{C}(16)-\mathrm{H}(17)$ & 120.3 \\
\hline $\mathrm{C}(17)-\mathrm{C}(16)-\mathrm{H}(17)$ & 120.3 \\
\hline$C(16)-C(17)-C(18)$ & $120.28(14)$ \\
\hline $\mathrm{C}(16)-\mathrm{C}(17)-\mathrm{H}(16)$ & 119.9 \\
\hline $\mathrm{C}(18)-\mathrm{C}(17)-\mathrm{H}(16)$ & 119.9 \\
\hline $\mathrm{C}(17)-\mathrm{C}(18)-\mathrm{C}(19)$ & $119.72(15)$ \\
\hline $\mathrm{C}(17)-\mathrm{C}(18)-\mathrm{H}(15)$ & 120.1 \\
\hline $\mathrm{C}(19)-\mathrm{C}(18)-\mathrm{H}(15)$ & 120.1 \\
\hline $\mathrm{C}(18)-\mathrm{C}(19)-\mathrm{C}(20)$ & $121.02(15)$ \\
\hline $\mathrm{C}(18)-\mathrm{C}(19)-\mathrm{H}(14)$ & 119.5 \\
\hline $\mathrm{C}(20)-\mathrm{C}(19)-\mathrm{H}(14)$ & 119.5 \\
\hline$C(19)-C(20)-C(15)$ & $118.18(13)$ \\
\hline$C(19)-C(20)-C(21)$ & $117.55(13)$ \\
\hline$C(15)-C(20)-C(21)$ & $123.82(13)$ \\
\hline $\mathrm{O}(1)-\mathrm{C}(21)-\mathrm{N}(4)$ & $122.02(14)$ \\
\hline
\end{tabular}




$\begin{array}{ll}\mathrm{O}(1)-\mathrm{C}(21)-\mathrm{C}(20) & 119.42(14) \\ \mathrm{N}(4)-\mathrm{C}(21)-\mathrm{C}(20) & 118.55(13) \\ \mathrm{C}(23)-\mathrm{C}(22)-\mathrm{C}(12) & 119.85(15) \\ \mathrm{C}(23)-\mathrm{C}(22)-\mathrm{H}(9) & 120.1 \\ \mathrm{C}(12)-\mathrm{C}(22)-\mathrm{H}(9) & 120.1 \\ \mathrm{C}(22)-\mathrm{C}(23)-\mathrm{C}(9) & 120.89(15) \\ \mathrm{C}(22)-\mathrm{C}(23)-\mathrm{H}(8) & 119.6 \\ \mathrm{C}(9)-\mathrm{C}(23)-\mathrm{H}(8) & 119.6 \\ \mathrm{~N}(4)-\mathrm{C}(24)-\mathrm{H}(13) & 109.5 \\ \mathrm{~N}(4)-\mathrm{C}(24)-\mathrm{H}(12) & 109.5 \\ \mathrm{H}(13)-\mathrm{C}(24)-\mathrm{H}(12) & 109.5 \\ \mathrm{~N}(4)-\mathrm{C}(24)-\mathrm{H}(11) & 109.5 \\ \mathrm{H}(13)-\mathrm{C}(24)-\mathrm{H}(11) & 109.5 \\ \mathrm{H}(12)-\mathrm{C}(24)-\mathrm{H}(11) & 109.5\end{array}$

Symmetry transformations used to generate equivalent atoms: 
Table S10. Anisotropic displacement parameters $\left(\AA^{2} \times 10^{3}\right)$ for 171133LT. The anisotropic displacement factor exponent takes the form: $-2 \pi^{2}\left[\mathrm{~h}^{2} \mathrm{a}^{* 2} \mathrm{U}^{11}+\ldots+2 \mathrm{hk} \mathrm{a}^{*} \mathrm{~b}^{*} \mathrm{U}^{12}\right]$

\begin{tabular}{lllllll}
\hline & $\mathrm{U}^{11}$ & $\mathrm{U}^{22}$ & $\mathrm{U}^{33}$ & $\mathrm{U}^{23}$ & $\mathrm{U}^{13}$ & $\mathrm{U}^{12}$ \\
\hline $\mathrm{O}(1)$ & $27(1)$ & $24(1)$ & $18(1)$ & $-2(1)$ & $7(1)$ & $6(1)$ \\
$\mathrm{N}(1)$ & $17(1)$ & $18(1)$ & $17(1)$ & $0(1)$ & $0(1)$ & $-1(1)$ \\
$\mathrm{N}(2)$ & $16(1)$ & $17(1)$ & $18(1)$ & $-2(1)$ & $0(1)$ & $-2(1)$ \\
$\mathrm{N}(3)$ & $14(1)$ & $16(1)$ & $16(1)$ & $-2(1)$ & $0(1)$ & $-2(1)$ \\
$\mathrm{N}(4)$ & $16(1)$ & $16(1)$ & $13(1)$ & $-1(1)$ & $0(1)$ & $2(1)$ \\
$\mathrm{C}(1)$ & $22(1)$ & $27(1)$ & $24(1)$ & $8(1)$ & $7(1)$ & $6(1)$ \\
$\mathrm{C}(2)$ & $17(1)$ & $21(1)$ & $30(1)$ & $4(1)$ & $4(1)$ & $0(1)$ \\
$\mathrm{C}(3)$ & $14(1)$ & $22(1)$ & $21(1)$ & $1(1)$ & $0(1)$ & $2(1)$ \\
$\mathrm{C}(4)$ & $13(1)$ & $20(1)$ & $18(1)$ & $1(1)$ & $3(1)$ & $3(1)$ \\
$\mathrm{C}(5)$ & $12(1)$ & $18(1)$ & $16(1)$ & $-2(1)$ & $0(1)$ & $1(1)$ \\
$\mathrm{C}(6)$ & $14(1)$ & $19(1)$ & $13(1)$ & $-1(1)$ & $2(1)$ & $1(1)$ \\
$\mathrm{C}(7)$ & $19(1)$ & $18(1)$ & $13(1)$ & $0(1)$ & $1(1)$ & $-1(1)$ \\
$\mathrm{C}(8)$ & $19(1)$ & $14(1)$ & $11(1)$ & $1(1)$ & $0(1)$ & $1(1)$ \\
$\mathrm{C}(9)$ & $15(1)$ & $14(1)$ & $15(1)$ & $2(1)$ & $-2(1)$ & $2(1)$ \\
$\mathrm{C}(10)$ & $18(1)$ & $16(1)$ & $16(1)$ & $0(1)$ & $1(1)$ & $2(1)$ \\
$\mathrm{C}(11)$ & $15(1)$ & $16(1)$ & $24(1)$ & $3(1)$ & $1(1)$ & $0(1)$ \\
$\mathrm{C}(12)$ & $20(1)$ & $14(1)$ & $24(1)$ & $-1(1)$ & $-6(1)$ & $-1(1)$ \\
$\mathrm{C}(13)$ & $20(1)$ & $25(1)$ & $21(1)$ & $-2(1)$ & $1(1)$ & $-2(1)$ \\
$\mathrm{C}(14)$ & $26(1)$ & $32(1)$ & $16(1)$ & $2(1)$ & $1(1)$ & $5(1)$ \\
$\mathrm{C}(15)$ & $18(1)$ & $15(1)$ & $12(1)$ & $-3(1)$ & $0(1)$ & $-2(1)$ \\
$\mathrm{C}(16)$ & $18(1)$ & $20(1)$ & $16(1)$ & $-4(1)$ & $-1(1)$ & $1(1)$ \\
$\mathrm{C}(17)$ & $26(1)$ & $16(1)$ & $19(1)$ & $-1(1)$ & $-3(1)$ & $3(1)$ \\
$\mathrm{C}(18)$ & $27(1)$ & $17(1)$ & $19(1)$ & $0(1)$ & $2(1)$ & $-4(1)$ \\
$\mathrm{C}(19)$ & $19(1)$ & $20(1)$ & $18(1)$ & $-1(1)$ & $2(1)$ & $-3(1)$ \\
$\mathrm{C}(20)$ & $18(1)$ & $16(1)$ & $11(1)$ & $-3(1)$ & $-1(1)$ & $-1(1)$ \\
$\mathrm{C}(21)$ & $14(1)$ & $15(1)$ & $17(1)$ & $-1(1)$ & $2(1)$ & $-3(1)$ \\
$\mathrm{C}(22)$ & $23(1)$ & $19(1)$ & $16(1)$ & $-3(1)$ & $-2(1)$ & $2(1)$ \\
$\mathrm{C}(23)$ & $16(1)$ & $21(1)$ & $18(1)$ & $0(1)$ & $0(1)$ & $1(1)$ \\
$\mathrm{C}(24)$ & $24(1)$ & $18(1)$ & $23(1)$ & $1(1)$ & $0(1)$ & $5(1)$ \\
& & & & & & \\
\hline
\end{tabular}


Table S11. Hydrogen coordinates $\left(x 10^{4}\right)$ and isotropic displacement parameters $\left(\AA^{2} \times 10^{3}\right)$ for 171133LT

\begin{tabular}{|c|c|c|c|c|}
\hline & $\mathrm{x}$ & $\mathrm{y}$ & $\mathrm{z}$ & $\mathrm{U}(\mathrm{eq})$ \\
\hline $\mathrm{H}(1)$ & 9281 & 17695 & 2138 & 29 \\
\hline $\mathrm{H}(18)$ & 9902 & 18152 & 3297 & 27 \\
\hline $\mathrm{H}(3)$ & 9710 & 15725 & 4224 & 23 \\
\hline $\mathrm{H}(6)$ & 7966 & 8819 & 3385 & 20 \\
\hline $\mathrm{H}(7)$ & 5448 & 8315 & 4277 & 20 \\
\hline $\mathrm{H}(10)$ & 4513 & 6170 & 3665 & 22 \\
\hline $\mathrm{H}(2)$ & 4858 & 4576 & 2569 & 23 \\
\hline $\mathrm{H}(4)$ & 8258 & 12357 & 2837 & 26 \\
\hline $\mathrm{H}(5)$ & 8437 & 14825 & 1921 & 30 \\
\hline $\mathrm{H}(17)$ & 9162 & 6688 & 5587 & 22 \\
\hline $\mathrm{H}(16)$ & 8563 & 4031 & 6263 & 25 \\
\hline $\mathrm{H}(15)$ & 7147 & 3994 & 6428 & 25 \\
\hline $\mathrm{H}(14)$ & 6337 & 6674 & 5952 & 23 \\
\hline $\mathrm{H}(9)$ & 6151 & 5127 & 2075 & 23 \\
\hline $\mathrm{H}(8)$ & 7084 & 7310 & 2672 & 22 \\
\hline $\mathrm{H}(13)$ & 5565 & 11975 & 4289 & 33 \\
\hline $\mathrm{H}(12)$ & 6314 & 12899 & 3841 & 33 \\
\hline $\mathrm{H}(11)$ & 6213 & 13483 & 4689 & 33 \\
\hline
\end{tabular}

